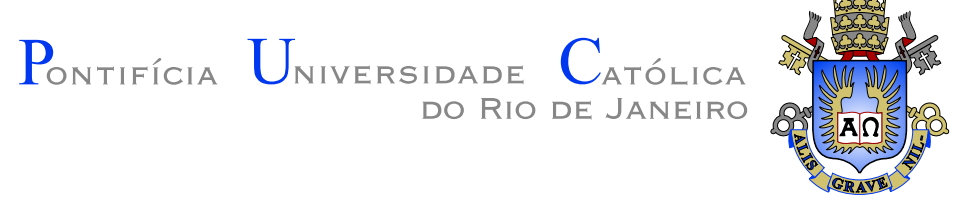

Vivian Barreiros Cosenza

\title{
Electromagnetic Characterization of Inhomogeneous Cylindrical Waveguides Using Mode-Matching-Based Methods
}

Thesis presented to the Programa de Pós-graduação em Engenharia Elétrica, do Departamento de Engenharia Elétrica da PUC-Rio in partial fulfillment of the requirements for the degree of Mestre em Engenharia Elétrica.

Advisor: Prof. Guilherme Simon da Rosa 


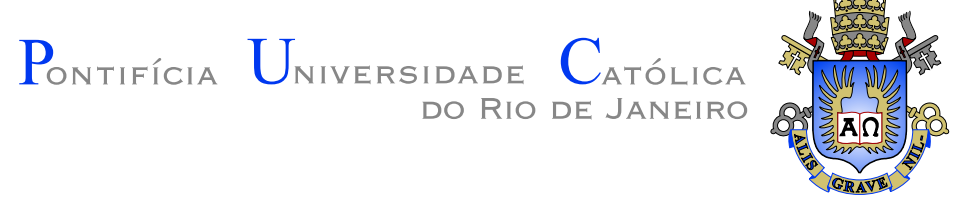

Vivian Barreiros Cosenza

\begin{abstract}
Electromagnetic Characterization of Inhomogeneous Cylindrical Waveguides Using Mode-Matching-Based Methods
\end{abstract}

Thesis presented to the Programa de Pós-graduação em Engenharia Elétrica da PUC-Rio in partial fulfillment of the requirements for the degree of Mestre em Engenharia Elétrica. Approved by the Examination Committee:

Prof. Guilherme Simon da Rosa

Advisor

Departamento de Engenharia Elétrica - PUC-Rio

Profa. Leni Joaquim de Matos

UFF

Prof. Rafael Abrantes Penchel

Unesp 
All rights reserved.

\section{Vivian Barreiros Cosenza}

She Received her B.S. degree in Telecommunication Engineering from Universidade Federal Fluminense, Rio de Janeiro, Brazil, in 2018. In 2018 she started her Master's degree in the Applied Electromagnetism in CETUC at the Pontifical Catholic University of Rio de Janeiro.

Bibliographic data

Barreiros Cosenza, Vivian

Electromagnetic Characterization of Inhomogeneous Cylindrical Waveguides Using Mode-Matching-Based Methods / Vivian Barreiros Cosenza; advisor: Guilherme Simon da Rosa. - 2020.

110 f: il. color. ; $30 \mathrm{~cm}$

Dissertação (mestrado) - Pontifícia Universidade Católica do Rio de Janeiro, Departamento de Engenharia Elétrica, 2020.

Inclui bibliografia

1. Engenharia Elétrica - Teses. 2. Engenharia de Telecomunicações - Teses. 3. Meio anisotrópico. 4. Meio estratificado. 5. Guia de ondas cilíndrico não homogêneo. 6. Método de casamento de modos. I. Simon da Rosa, Guilherme. II. Pontifícia Universidade Católica do Rio de Janeiro. Departamento de Engenharia Elétrica. III. Título. 


\section{Acknowledgments}

I would like first to thank my advisor Guilherme Simon da Rosa for their consistent assistance, guidance, comments, and suggestions during this project.

Also I am grateful to professor Glaucio Lima Siqueira, for accepted me in my first year at PUC and for the initial supervision.

I would like to thank to PUC-Rio for the exemption of scheel fess offered by the Programa de Pós-Graduação em Engenharia Elétrica.

This study was financed in part by the Coordenação de Aperfeiçoamento de Pessoal de Nível Superior - Brasil (CAPES) - Finance Code 001.

Finally, I must thank my parents Tânia and Marcos, to my brother Rafael, to Ricardo and Ewellym, for the continuous encouragement throughout these years of study. This accomplishment would not have been possible without you. 


\section{Abstract}

Barreiros Cosenza, Vivian; Simon da Rosa, Guilherme (Advisor). Electromagnetic Characterization of Inhomogeneous Cylindrical Waveguides Using Mode-Matching-Based Methods. Rio de Janeiro, 2020. 110p. Dissertação de Mestrado - Departamento de Engenharia Elétrica, Pontifícia Universidade Católica do Rio de Janeiro.

Many devices and structures used to guide electromagnetic waves are conformal with the cylindrical coordinates. Sensitive applications of microwave engineering and integrated optical devices often use non-homogeneous, anisotropic and dissipative materials, so that the research for robust and accurate computational models is a topic of remarkable interest for Electrical Engineering. This work presents a semi-analytical technique for solving boundary-value problems associated with cylindrical, anisotropic, and non-homogeneous waveguides. Our methodology allows us to model structures with radial layers, with uniaxial anisotropy, and with losses. The proposed solution starts from Maxwell's equations for time-harmonic electromagnetic fields and employs a modal expansion in terms of the Bessel-Fourier series. The eigenvalues associated with the problem are obtained using the winding number method, in which several approaches for calculating complex-plane contour integrals are explored in detail. In order to properly analyze the junctions between sections of stratified waveguides, we employ a mode-matching technique based on the conservation of the Reaction of the fields. Our formulation can handle the effects of excitation and coupling between pure modes (TM, TE, and TEM) in homogeneous waveguides, as well as hybrid modes in complex structures. A series of numerical results are presented and show the capacity of the methodology developed here to correctly characterize cylindrical structures composed of complex media (inhomogeneous, anisotropic, and dissipative) in a robust and computationally-efficient fashion if compared to other conventional computational electromagnetic techniques.

\section{Keywords}

Anisotropic media; Multilayered media; Inhomogeneous cylindrical waveguide; Mode-matching technique. 


\section{Resumo}

Barreiros Cosenza, Vivian; Simon da Rosa, Guilherme. Caracterização Eletromagnética de Guias de Onda Cilíndricos Não Homogêneos usando o Método do Casamento de Modos. Rio de Janeiro, 2020. 110p. Dissertação de Mestrado - Departamento de Engenharia Elétrica, Pontifícia Universidade Católica do Rio de Janeiro.

Muitos dispositivos e estruturas empregados para guiar ondas eletromagnéticas apresentam conformidade cilíndrica. Aplicações sensíveis de engenharia de micro-ondas e de dispositivos ópticos integrados empregam, muitas vezes, materiais não homogêneos, anisotrópicos e dissipativos, de modo que a pesquisa por modelos computacionais robustos e acurados é um tópico de notável interesse para a Engenharia Elétrica. Este trabalho apresenta uma técnica semianalítica para resolver problemas de valor de contorno associados a guias de onda cilíndricos, anisotrópicos e não homogêneos. Nossa metodologia permite modelar estruturas com camadas radiais, com anisotropia uniaxial, e com perdas. A solução proposta parte das equações de Maxwell para campos harmônicos no tempo, e emprega uma expansão modal em termos da série de Bessel-Fourier. Os autovalores associados ao problema são obtidos por meio do método do winding number, em que diversas abordagens para o cálculo das integrais de caminho no plano complexo são exploradas. Para analisar junções entre guias de ondas estratificados, empregamos a técnica de casamento de modos baseada na conservação da Reação dos campos. Nossa formulação é capaz de avaliar os efeitos da excitação e do acoplamento entre modos puros (TM, TE, e TEM) em guias de ondas homogêneos, bem como dos modos híbridos em estruturas complexas. Uma série de resultados numéricos são apresentados e mostram a capacidade da metodologia desenvolvida nesta pesquisa para caracterizar corretamente estruturas cilíndricas compostas por meios complexos (não homogêneos, anisotrópicos e dissipativos) de forma robusta e computacionalmente eficiente se comparado com outras técnicas convencionais de eletromagnetismo computacional.

\section{Palavras-chave}

Meio anisotrópico; Meio estratificado; Guia de ondas cilíndrico não homogêneo; Método de casamento de modos. 


\section{Table of contents}

1 Introduction $\quad 12$

$\begin{array}{lll}1.1 & \text { General Introduction } & 12\end{array}$

$\begin{array}{lll}1.2 & \text { Scientific Contributions } & 15\end{array}$

$\begin{array}{lll}1.3 & \text { Dissertation Organization } & 16\end{array}$

2 Electromagnetic Fields in Cylindrical Waveguides $\quad 17$

$\begin{array}{lll}2.1 & \text { Homogeneous Waveguide } & 18\end{array}$

$\begin{array}{lll}2.1 .1 & \text { Axial Fields } & 21\end{array}$

$\begin{array}{lll}2.1 .2 & \text { Transversal Fields } & 22\end{array}$

2.1.3 Circular and Coaxial Waveguides 23

2.1.4 Root-finding in the $k_{\rho}$ plane 24

$\begin{array}{ll}2.2 & \text { Inhomogeneous Waveguide } \\ 2\end{array}$

2.2.1 Root-finding in the $k_{z}$ plane 29

2.3 Validation 32

3 Mode-Matching Method for Cylindrically-Layered Waveguides $\quad 55$

$\begin{array}{lll}3.1 & \text { Formulation } & 57\end{array}$

3.2 Reaction Integrals $\quad 60$

3.2.1 Solutions for the TEM-mode 62

3.2.2 Regions with Arbitrary Layers 66

3.3 Cascading Generalized Scattering Matrices 67

$\begin{array}{lll}3.4 & \text { Validation } & 69\end{array}$

$\begin{array}{lll}3.4 .1 & \text { Coaxial Junctions } & 69\end{array}$

3.4.2 Coaxial to Circular Junctions 84

3.4.3 Circular to Circular Junctions 88

3.4.4 Structures with Multiple Junctions 90

$\begin{array}{lll}4 & \text { Conclusion } & 99\end{array}$

$\begin{array}{ll}\text { Bibliography } & 101\end{array}$ 


\section{List of figures}

Figure 1.1 Example of a structure for our problem. The radial domain is truncated by a perfect electric conductor (PEC).

Figure 2.1 Cylindrical coordinate system.

Figure 2.2 Coaxial waveguide. The shaded areas represent a perfect electric conductor (PEC) or a perfect magnetic conductor (PMC). 24

Figure 2.3 Region of search on the $k_{z}$-complex plane, where $k_{z}^{\prime}$ is the real and $k_{z}^{\prime \prime}$ is the imaginary part of $k_{z}$, respectively.

Figure 2.4 Inhomogeneous coaxial waveguide with $N$ different layers. 27

Figure 2.5 Region of search on the $k_{z}$-complex plane, and the contours $C_{1}, C_{2}$ and $C_{3}$ used in the calculation of the winding number.

Figure 2.6 First nine wavenumbers $k_{z}$ for waveguide 1 at different operating frequencies.

Figure 2.7 Normalized $E_{z}$ and $E_{\rho}$ fields of the first propagating mode in waveguide 1 at different operating frequencies.

Figure 2.8 Normalized $E_{z}$ and $E_{\phi}$ fields of the first propagating modes in waveguide 1 at different operating frequencies.

Figure 2.9 Normalized $E_{z}$ and $E_{\rho}$ fields of the first propagating mode in waveguide 1 in $160 \mathrm{GHz}$. Discontinuities of $E_{\rho}$ are smaller for higher frequencies.

Figure 2.10 First nine wavenumbers $k_{z}$ for waveguide 2 at different operating frequencies.

Figure 2.11 Normalized $E_{z}$ and $E_{\rho}$ fields of the first propagating mode in waveguide 2 at different operating frequencies.

Figure 2.12 Normalized $E_{z}$ and $E_{\phi}$ fields of the first propagating modes in waveguide 2 at different operating frequencies.

Figure 2.13 Normalized $E_{z}$ and $E_{\rho}$ fields of the first propagating mode in waveguide 2 at $160 \mathrm{GHz}$. Discontinuities of $E_{\rho}$ are smaller for higher frequencies.

Figure 2.14 Normalized $E_{\phi}$ fields of the main propagating mode in a three-layer circular waveguide at $100 \mathrm{GHz}$. A dielectric media (with $\epsilon_{r}=3$ ) is placed in the innermost, middle, and outermost radial layers.

Figure 2.15 Normalized $E_{\phi}$ field component of the main propagating mode in a (virtual) three-layer circular waveguide at $100 \mathrm{GHz}$. The outermost radial layer is filled with a material characterized by the electric permittivity $\epsilon_{r}$.

Figure 2.16 First nine wavenumbers $k_{z}$ for waveguide 3 at different operating frequencies.

Figure 2.17 Normalized $E_{z}$ and $E_{\rho}$ fields of the first propagating mode in waveguide 3 at different operating frequencies.

Figure 2.18 Normalized $E_{z}$ and $E_{\phi}$ fields of the first propagating modes in waveguide 3 at different operating frequencies. 
Figure 2.19 First nine wavenumbers $k_{z}$ for waveguide 4 at different operating frequencies.

Figure 2.20 Normalized $E_{z}$ and $E_{\rho}$ fields of the first propagating mode in waveguide 4 at different operating frequencies.

Figure 2.21 Normalized $E_{z}$ and $E_{\phi}$ fields of the first propagating modes in waveguide 4 at different operating frequencies.

Figure 2.22 Normalized $E_{z}$ and $E_{\phi}$ fields in an anisotropic waveguide 4 at different operating frequencies.

Figure 2.23 Normalized $E_{z}$ and $E_{\phi}$ fields of simulation 1 at $80 \mathrm{GHz} . \quad 52$

Figure 2.24 Normalized $E_{z}$ and $E_{\phi}$ fields of simulation 2 at $80 \mathrm{GHz} . \quad 53$

Figure 2.25 Normalized $E_{\rho}$ field component of the main propagating mode in waveguide 5 at $20 \mathrm{GHz}$. The value of $\epsilon_{r}$ were varied according to Table 2.11, to simulate different levels of anisotropy. 54

Figure 3.1 Junction of two homogeneous coaxial waveguides.

Figure 3.2 Waveguide discontinuity cases where the TEM mode occur: (a) homogeneous to inhomogeneous junction, (b) inhomogeneous to homogeneous junction and (c) homogeneous to homogeneous junction.

Figure 3.3 Junction between waveguides with non-homogeneous media.

Figure 3.4 Structure with three regions.

Figure 3.5 Flowchart of the developed algorithm.

Figure 3.6 Geometry of junction 1. The darker region represents the inner layers with $\epsilon_{r}=2.55$.

Figure 3.7 Reflection coefficient of junction 1 from 0.5 to $45 \mathrm{GHz}$.

Figure 3.8 Geometry of junction 2 .

Figure 3.9 Reflection coefficient of junction 2 from 0.5 to $50 \mathrm{GHz}$ with two layers in each region.

Figure 3.10 Reflection coefficient of modified junction 2 from 0.5 to $50 \mathrm{GHz}$ with three layers in region 1 and four layers in region $2 . \quad 74$

Figure 3.11 Geometry of junction 3.

Figure 3.12 Reflection coefficient of junction 3 from 0.5 to $50 \mathrm{GHz} . \quad 76$

Figure 3.13 Geometry of junction 4 . 77

Figure 3.14 Reflection coefficient of junction 4 from 0.5 to $50 \mathrm{GHz} . \quad 77$

Figure 3.15 Reflection coefficient of junction 5 from 0.5 to $50 \mathrm{GHz} . \quad 79$

Figure 3.16 Geometry of junction 6 . 80

Figure 3.17 Reflection coefficient of junction 6 from 0.5 to $50 \mathrm{GHz} . \quad 80$

Figure 3.18 Geometry of junction 7 . 81

Figure 3.19 Reflection coefficient of junction 7 from 0.5 to $50 \mathrm{GHz} . \quad 82$

Figure 3.20 Geometry of junction 8 . The filled area in region 2 represents the outermost radial layer with $\epsilon_{r 2}=3.8$. 83

Figure 3.21 Reflection coefficient of junction 8, for different materials. 84

Figure 3.22 Geometry of junction 9.

Figure 3.23 Reflection coefficient of junction $9 . \quad 86$

Figure 3.24 Geometry of junction $10 . \quad 87$

$\begin{array}{ll}\text { Figure 3.25 Reflection coefficient of junction } 10 . & 87\end{array}$

Figure 3.26 Reflection coefficient of junction $11 . \quad 88$

Figure 3.27 Reflection coefficient of junction $12 . \quad 89$ 
Figure 3.28 Geometry of structure 1. 91

Figure 3.29 Reflection coefficient of structure $1 . \quad 91$

Figure 3.30 Geometry of structure 2. 93

Figure 3.31 Reflection coefficient of structure 2. 93

Figure 3.32 Reflection coefficient of structure 2 considering different levels of anisotropy. $\quad 94$

Figure 3.33 Geometry of structure 3. 95

Figure 3.34 Reflection coefficient of structure 3. 96

Figure 3.35 Reflection coefficient of structure 3 considering different $\begin{array}{ll}\text { levels of anisotropy. } & 96\end{array}$

$\begin{array}{ll}\text { Figure 3.36 Geometry of structure } 4 . & 97\end{array}$

Figure 3.37 Reflection coefficient of structure $4 . \quad 98$ 


\section{List of tables}

Table 2.1 Dimensions and parameters of the reference waveguide. Other constitutive parameters are equal to those of vacuum.

Table 2.2 Number of zeros of reference waveguide for the different methods presented.

Table 2.4 Dimensions and parameters of waveguide 1. Other constitutive parameters are equal to those of vacuum.

Table 2.5 Dimensions and parameters of waveguide 2. Other constitutive parameters are equal to those of vacuum.

Table 2.6 Dimensions and parameters of waveguide 3.

Table 2.7 Dimensions and parameters of waveguide 4 .

Table 2.8 Dimensions and parameters of waveguide 4 with anisotropic layers.

Table 2.9 Dimensions and parameters of waveguides used in simulation 1 and 2 .

Table 2.10 Dimensions and parameters of waveguide 5 with anisotropic layers.

Table 2.11 Values of $\epsilon_{r z}$ for Waveguide 5.

$\begin{array}{lll}\text { Table 3.1 Dimensions and parameters of junction } 1 . & 71\end{array}$

Table 3.2 Dimensions and parameters of junction $2 . \quad 73$

Table 3.3 Dimensions and parameters of modified junction 2. $\quad 73$

Table 3.4 Dimensions and parameters of junction $3 . \quad 75$

Table 3.5 Dimensions and parameters of junction $4 . \quad 76$

$\begin{array}{lll}\text { Table 3.6 Dimensions and parameters of junction } 5 . & 78\end{array}$

Table 3.7 Dimensions and parameters of junction $6 . \quad 79$

Table 3.8 Dimensions and parameters of junction $7 . \quad 81$

Table 3.9 Dimensions and parameters of junction $8 . \quad 83$

Table 3.10 Values of $\epsilon_{r}$ for different materials [1]. 83

Table 3.11 Cutoff frequencies (in $\mathrm{GHz}$ ) in region 2 for junction 8, with different values of $\epsilon_{r}$.

Table 3.12 Dimensions and parameters of junction 9.

Table 3.13 Dimensions and parameters of junction $10 . \quad 86$

Table 3.14 Dimensions and parameters of junction 11.

Table 3.15 Dimensions and parameters of junction 12.

Table 3.16 Dimensions and parameters of structure $1 . \quad 90$

Table 3.17 Dimensions and parameters of structure 2. 92

Table 3.18 Dimensions and parameters of structure 3. 95

Table 3.19 Dimensions and parameters of structure 4. 97 


\section{Introduction}

\section{1}

\section{General Introduction}

The study of inhomogeneous and anisotropic materials has become extremely important over the past years due to its uses on novel microwave and optical devices. It is essential to comprehend the propagation characteristics of the field in these complex media in order to develop more robust and reliable electromagnetic devices. Theoretical analysis and simulation models are essentials today for supporting telecommunication projects on initial stages, before its effective implementation.

Several different approaches can be used for addressing the electromagnetic field propagation on non-homogeneous materials, including field experiments and mainly supported with numerical models. The analysis of a multilayered media is a topic of interest of many recent researches [2-9] that investigate artificially engineered materials. Scattering and propagating electromagnetic models are popular in many applications such as: nondestructive testing [10,11], structural monitoring of components [12], design and evaluation of well-logging sensors in the oil and gas industry [13-16] underwater electromagnetic communication [17], among others.

Another relevant point of study is related to anisotropic media that become widely used in microwave engineering and integrated optics [18]. There are many technological advancements that were achieved by the employment of specialized anisotropic materials, e.g., optical fibers with preferred opticalaxis orientation, quasi-transparent antenna radomes, anisotropic films and substrates for microstrip antennas [19].

There is today an emergent effort for developing novel characterization methodologies for inhomogeneous and anisotropic media. Electromagnetic models for simulating anisotropy materials are used today for solving complex cutting-edge issues in geophysical exploration [12, 20-22], optical fiber communication, integrated optics [23] and wave absorbents design [24]. Field solutions for Maxwell's equations in uniaxial and biaxial anisotropic media were explored in the works in [25-31] using analytic, semi-analytic and pure- 
numerical (brute-force) techniques.

Propagation of electromagnetic waves along cylindrical waveguides filled with anisotropic and inhomogeneous material is also recurrent topic of research. This class of structure, composed in general by radial and longitudinal (axial) layers is used in the microwave guided devices. For example, such cylindrically conforming structures have applications for the analysis and design of coaxial horn antennas $[32,33]$ as well as the modeling of measurement cell for the characterization of complex-valued tensor constitutive parameters (permeability $\overline{\bar{\mu}}$ and permittivity $\overline{\bar{\epsilon}}$ ) [34-40].

Coaxial measurement cells are comprised by the junctions of sections of coaxial waveguides holding a sample under analysis. This device can be used for finding the permeability and the permittivity of a given material by using direct measurements of the scattering (S) parameters on the input and output ports. The simplicity of the geometry and the wide-frequency range of the TEM (or quasi-TEM) fields confer to this structure a excellent method for characterizing the samples. The methodology consists on an inversion problem with two steps: 1) measuring the S-parameters over the frequency, and 2) an optimization algorithm where the direct problem (with assumed values of permeability and the permittivity) is solved until the simulation scattering characteristics fits to the measured ones. The main concern of this research is on modeling the direct problem in a robust and computationally-efficient fashion.

The problem at hand can be solved by standard computational electromagnetic techniques based on brute-force numerical discretization of Maxwell's equations. However, low-cost algorithms are demanded if we want to proceed to an inverse problem. In the frequency domain, numerical solvers based on the finite-element method (FEM) [41, 42] or on the frequency-domain transmission line matrix (FDTLM) [36] are popular choices. In time domain, the finite-difference time-domain (FDTD) [43, 44] as well others transientbased solutions for Maxwell's equations can be effectively used for modeling a measuring cell [45].

Although the time-domain-based techniques can be used in a broad frequency band, such solutions suffer from poor accuracy [46]. If it is desired more resolution in the measurements, the frequency domain methods are more appropriated.

A popular semi-analytic approach for modeling simple waveguided structures is the mode-matching technique (MMT). Recently, this classic method was revisited and generalized for modeling fields along waveguide filled with non-homogeneous materials $[14,15,47,48]$. As the geometry of the coaxial 
cells we are interested presents only simple cylindrical-conforming boundaries, we will employ a MMT-based methodology in this works.

The mode-matching is a semi-analytical method, thereby, it requires lower computational resources than other numerical methods as FEM and FDTD. In the MMT, electromagnetic fields are expanded into a modal series to represent each sector of a waveguide, and coupling integrals are established to match the boundary conditions between each waveguide discontinuity. These coupling integrals can be arranged as a generalized scattering matrix (GSM) for each discontinuity. By the proper cascading of the GSMs, we can characterize an entire longitudinal guided structure. This method requires knowledge of modal eigenfunctions and the associated eigenvalues that satisfy the boundaryvalue problem on a uniform-cross-section waveguide. In our approach, the employment of a judicious Bessel-Fourier series will result in a characteristic equation for each region of the structure. In the treatment of complex lossy media, complex-valued eigenvalues will arise; they can be solved via some the Cauchy integral formula [28, 49] and root-finding [50] algorithms.

In this work, we will present a comprehensive mathematical formulation for the electromagnetic characterization of coaxial and circular guidedstructures. These structures are comprised of junctions of waveguides, where each one can be filled by inhomogeneous, anisotropic, and lossy materials. To put it into context, consider the simplified geometry depicted in Fig. 1.1. Our strategy is to employ the MMT for coupling each sub-domain comprising a uniform waveguide; in this example, only three waveguide regions are needed. The mode-matching enforcement will be over the cross-sectional aperture between a waveguide its adjacent ones. The radial profile of the anisotropic constitutive parameters will be approximated by layered media, and the number of radial layers is arbitrary in each waveguide sub-domain. In general, recursive applications of the MMT will allow us to handle an arbitrary number of longitudinal waveguide junctions.

The fields inside each sub-domain (waveguide region) of the structures will be derived through Maxwell's equations via the linear combination of pure transverse magnetic (TM), transverse electric (TE), and transverse electromagnetic (TEM) fields (to the longitudinal directions) in homogeneousfilled waveguides. In the case of non-homogeneous media, a set of hybrid fields will be employed for completeness. The radial boundary conditions will be enforced via compact reflection and transmission matrices. Next, appropriated eigenvalues will be found by using the winding number method associated with the Muller's root-finding. Finally, in view of the MMT, a set of coupling integrals will be presented with the corresponding scattering parameters of 


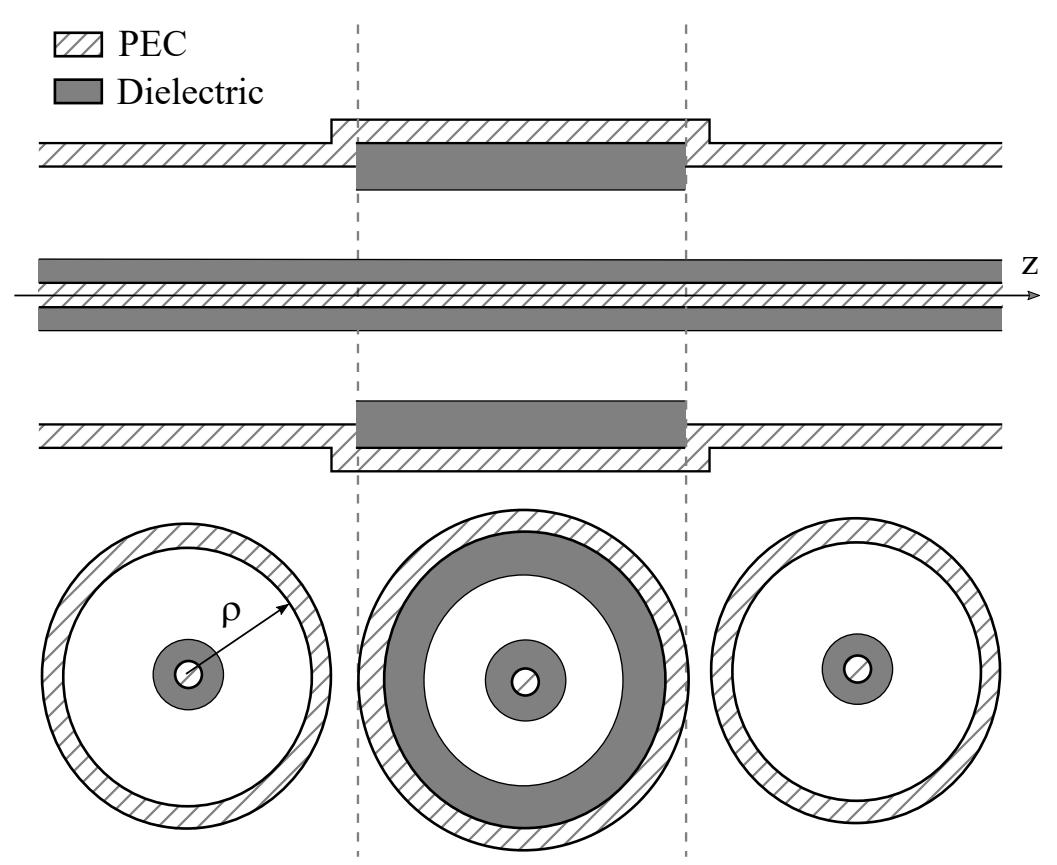

Figure 1.1: Example of a structure for our problem. The radial domain is truncated by a perfect electric conductor (PEC).

each waveguide junction. We were able to found closed-form solutions for all of the associated coupling integrals.

\section{2}

\section{Scientific Contributions}

In this work, we purpose a mode-matching-based methodology for the electromagnetic analysis of inhomogeneous cylindrical-conforming waveguides filled with an generic uniaxial and layered lossy media. In other words, our solution is able to model lossy or lossless, anisotropic or isotropic, and layered or homogeneous media. The waveguides considered can be coaxial (with a inner concentric conductor) or circular, supporting as many radial layers as needed. In the works $[18,33]$ mode-matching formulations were presented but restricted to only two radial isotropic layers. We introduced here a formulation able to describe fields in multi-layered waveguides in complex scenarios.

To acquire the eigenvalues, the winding number method was applied to determine the number of zeros of our characteristic equation, and Muller's method is used to obtain it, as mentioned above. In this work, two approaches for computing the winding number were explored, generalizing the formulation in [51].

Additionally, specialized coupling integrals where analytically solved here for the coupling between homogeneous and non-homogeneous waveguides. Such 
results are particular forms of Lommel integrals associated with the TEM modal field inherent of homogeneous coaxial waveguides. To the best of our knowledge, this is the first time that such kind of problem is properly solved in a closed-form.

The scientific contributions of this work are summarized below:

- The use of a semi-analytical method (MMT) to evaluate multiple layers junctions, which not necessarily have the same number of layers in each region;

- The composition of the dielectric materials that fill these layers may be lossy and/or anisotropic;

- The presentation of two new ways to calculate the winding number and determine the number of zeros of a characteristic equation;

- The introduction of new closed-form equations for considering the coupling of a TEM with TM or hybrid modes in the MMT.

\section{3}

\section{Dissertation Organization}

The rest of this dissertation is organized as follows. In Chapter 2, a derivation of fields inside homogeneous and inhomogeneous cylindrical waveguides are presented. The derivations of the characteristic equation of circular and coaxial waveguides are then demonstrated. The methods used to determine the modal propagating constants in the coaxial and circular waveguides for radially homogeneous and inhomogeneous scenarios are derived and a series of numerical results are also presented.

In Chapter 3, the mode-matching formulation is established via the conservation of the reactions of the fields. Closed-form results are presented for the coupling integrals associated to generic hybrid modes. Specialized results are also presented for the coupling between a TEM with TEM modes, as well as if a TEM with TM or hybrid modes. The details of the computation of generalizes scattering matrices are also presented. Simulation results comparing the Sparameters predicted by our method versus FDTD-based and FEM-based ones testify that our formalism can correctly characterize cylindrical structures composed by complex media.

Finally, Chapter 4 compiles the most important results obtained in this work and suggests future research directions. 


\section{2 \\ Electromagnetic Fields in Cylindrical Waveguides}

Coaxial and circular transmission lines and cavities are popular configurations for telecommunications systems. Circular waveguides are easy to produce and have gained attention for long distances propagation for $\mathrm{TE}_{0 n}$ modes, especially at millimeter-wave range, because its attenuation coefficient decreasing with frequency [1]. Homogeneous coaxial waveguides support in addition to TE and TM modes the transmission-line TEM mode.

In a typical single-mode waveguide, the entire field propagation can be characterized by one propagating mode; called fundamental mode. In such configuration, all the other modes are evanescent and could be neglected. However, when we are close to a waveguide discontinuity or near the source region, these high-order modes become significant to correctly describe the fields even in a single-propagating-mode frequency range. It is necessary to consider, then, an infinity set of non-propagating modes for completeness [52]. For practical applications, as far as we are way from critical regions (near the sources or waveguide junctions) the fundamental mode becomes dominant again.

In this chapter, we will present a mathematical formalism for representing electromagnetic fields in an inhomogeneous anisotropic cylindrical structure. The cylindrical coordinates are used to solve problems with cylindricalconforming boundaries. The problem at hand uses coaxial and circular waveguides, and then, it will be necessary to express the fields in a cylindrical coordinate system, as depicted in Fig. 2.1.

Non-homogeneous circular and coaxial waveguides have been studied over the years because its importance in microwave and antenna engineering. Different techniques are used to solve this boundary-value problem. In [53] and [54], a direct numerical integration is employed to solve electromagnetic dispersion in a coaxial cable. It also uses a shooting method to the solve Maxwell's equations. A perturbation method was used in [55] to approximate a solution to the wave equation. These methods however, suffer with numerical problems and/or inaccuracy in the modeling of large-contrast layered waveguides.

We will employ here a semi-analytic technique to solve our problem by avoiding discretization of the spatial domain via a mode-matching-based 

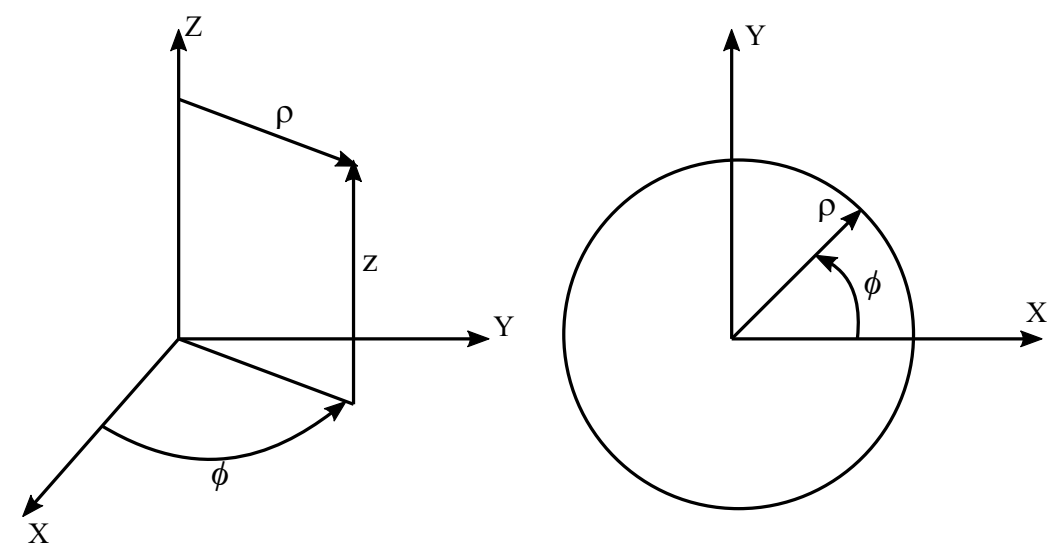

Figure 2.1: Cylindrical coordinate system.

solution. A complete eigenfunction expansion and a numerically efficient solution for the associated eigenvalues will be presented on the grounds of the winding number method.

From Maxwell's equations, we will derive full-wave closed-form field solutions for modeling a radially-layered circular waveguide. This waveguide can be filled with lossy and uniaxially anisotropic media. As a special case, if the innermost radial layer becomes a PEC, our solution will reproduce a radially layered coaxial waveguide.

First, we present the field solution in a homogeneous uniaxially anisotropic media. A computationally efficient method is used to the treatment of a stratified waveguide by using the homogeneous field solutions as a basis. Our approach is a generalization of that presented in $[51,56]$. The timeharmonic dependence in the form $e^{-i \omega t}$ is assumed and omitted.

\section{1}

\section{Homogeneous Waveguide}

Maxwell's equations for a homogeneous and anisotropic medium are as follows:

$$
\begin{aligned}
& \boldsymbol{\nabla} \times \mathbf{E}=i \omega \overline{\bar{\mu}} \cdot \mathbf{H}, \\
& \nabla \times \mathbf{H}=-i \omega \overline{\bar{\epsilon}} \cdot \mathbf{E}+\mathbf{J}, \\
& \nabla \cdot(\overline{\bar{\epsilon}} \cdot \mathbf{E})=\varrho, \\
& \nabla \cdot(\overline{\bar{\mu}} \cdot \mathbf{H})=0,
\end{aligned}
$$


where $\mathbf{E}$ and $\mathbf{H}$ are the electric and magnetic fields, respectively, produced by the volumetric current density $\mathbf{J}$. The permeability and permittivity tensors in cylindrical coordinates $(\rho, \phi, z)$ are given by

$$
\overline{\bar{\epsilon}}=\left[\begin{array}{ccc}
\epsilon_{s} & 0 & 0 \\
0 & \epsilon_{s} & 0 \\
0 & 0 & \epsilon_{z}
\end{array}\right] \quad \text { and } \quad \overline{\bar{\mu}}=\left[\begin{array}{ccc}
\mu_{s} & 0 & 0 \\
0 & \mu_{s} & 0 \\
0 & 0 & \mu_{z}
\end{array}\right]
$$

where the subscript $s$ is used here to indicate the coordinates transversal to $z$, $\epsilon_{\{s, z\}}=\epsilon_{0} \epsilon_{r\{s, z\}}+i \sigma_{\{s, z\}} \omega, \mu_{\{s, z\}}=\mu_{0} \mu_{r\{s, z\}}$, and $\sigma$ is the electrical conductivity of the medium. Decomposing the vector fields in transversal and axial (or longitudinal to $z$ ) components, we can write

$$
\mathbf{G}=\mathbf{G}_{s}+\hat{z} G_{z}
$$

where $G=\{E, H\}$ and

$$
\boldsymbol{\nabla}=\boldsymbol{\nabla}_{s}+\hat{z} \frac{\partial}{\partial z}
$$

The transverse differential operator $\nabla_{s}$ is expressed in the form

$$
\nabla_{s}=\hat{\rho} \frac{\partial}{\partial \rho}+\hat{\phi} \frac{1}{\rho} \frac{\partial}{\partial \phi} .
$$

By taking the curl operator of equation (2-1) and projecting its result in $\hat{z}$, we can obtain

$$
\hat{z} \cdot[\nabla \times(\nabla \times \mathbf{E})]=i \omega \hat{z} \cdot[\nabla \times(\overline{\bar{\mu}} \cdot \mathbf{H})] .
$$

The right-hand side of the above expression might be rewritten as

$$
i \omega \hat{z} \cdot[\boldsymbol{\nabla} \times(\overline{\bar{\mu}} \cdot \mathbf{H})]=i \omega \mu_{s} \hat{z} \cdot(\boldsymbol{\nabla} \times \mathbf{H}) .
$$

By using equation (2-2), it is possible to write

$$
i \omega \hat{z} \cdot[\nabla \times(\overline{\bar{\mu}} \cdot \mathbf{H})]=i \omega \mu_{s}\left(-i \omega \epsilon_{z} E_{z}\right)
$$

and therefore, equation (2-9) becomes

$$
\hat{z} \cdot[\nabla \times(\nabla \times \mathbf{E})]=\omega^{2} \mu_{s} \epsilon_{z} E_{z} .
$$

We can simplify the left-hand side of the above equation by using the vector identity

$$
\nabla \times \nabla \times \mathbf{E}=\nabla(\nabla \cdot \mathbf{E})-(\nabla \cdot \nabla) \mathbf{E} .
$$


From (2-4), we can derive

$$
\begin{aligned}
\boldsymbol{\nabla} \cdot(\overline{\bar{\mu}} \cdot \mathbf{H}) & =0 \\
\left(\boldsymbol{\nabla}_{s}+\hat{z} \frac{\partial}{\partial z}\right) \cdot\left(\mu_{s} \mathbf{H}_{s}+\hat{z} \mu_{z} H_{z}\right) & =0 \\
\boldsymbol{\nabla}_{s}\left(\mu_{s} \cdot \mathbf{H}_{s}\right)+\mu_{z} \frac{\partial H_{z}}{\partial z} & =0 \\
\mu_{s} \boldsymbol{\nabla} \cdot \mathbf{H}-\mu_{s}\left(1-\frac{\mu_{z}}{\mu_{s}}\right) \frac{\partial H_{z}}{\partial z} & =0 \\
\therefore \boldsymbol{\nabla} \cdot \mathbf{H} & =\left(1-\frac{\mu_{z}}{\mu_{s}}\right) \frac{\partial H_{z}}{\partial z} .
\end{aligned}
$$

A dual equation can be obtained for the electric field, that is,

$$
\boldsymbol{\nabla} \cdot \mathbf{E}=\left(1-\frac{\epsilon_{z}}{\epsilon_{s}}\right) \frac{\partial E_{z}}{\partial z}
$$

By using (2-13) and (2-15), we can obtain

$$
\begin{aligned}
\hat{z} \cdot[\boldsymbol{\nabla} \times(\boldsymbol{\nabla} \times \mathbf{E})] & =\hat{z} \cdot \boldsymbol{\nabla}(\boldsymbol{\nabla} \cdot \mathbf{E})-\hat{z} \cdot \nabla^{2} \mathbf{E} \\
& =\left(1-\frac{\epsilon_{z}}{\epsilon_{s}}\right) \frac{\partial^{2} E_{z}}{\partial z^{2}}-\nabla^{2} E_{z}
\end{aligned}
$$

where $\nabla^{2}$ is the scalar Laplacian operator. In cylindrical coordinates, it is expressed by

$$
\nabla^{2}=\frac{1}{\rho} \frac{\partial}{\partial \rho}\left(\rho \frac{\partial}{\partial \rho}\right)+\frac{1}{\rho} \frac{\partial^{2}}{\partial \phi^{2}}+\frac{\partial^{2}}{\partial z^{2}}
$$

Substituting (2-16) in (2-12) results in

$$
\begin{aligned}
\left(1-\frac{\epsilon_{z}}{\epsilon_{s}}\right) \frac{\partial^{2} E_{z}}{\partial z^{2}}-\nabla^{2} E_{z} & =\omega^{2} \mu_{s} \epsilon_{z} E_{z} \\
\therefore \quad\left(\nabla_{s}^{2}+\frac{\epsilon_{z}}{\epsilon_{s}} \frac{\partial^{2}}{\partial z^{2}}+\omega^{2} \mu_{s} \epsilon_{z}\right) E_{z} & =0 .
\end{aligned}
$$

By using duality, the above allows us to obtain a wave equation for the axial magnetic field according to:

$$
\left(\nabla_{s}^{2}+\frac{\mu_{z}}{\mu_{s}} \frac{\partial^{2}}{\partial z^{2}}+\omega^{2} \mu_{z} \epsilon_{s}\right) H_{z}=0 .
$$

Equations (2-18) and (2-19) are wave functions, or Helmholtz wave equations for the axial electric and magnetic fields, respectively. They can be rewritten in the following compact way:

$$
\left[\frac{1}{\rho} \frac{\partial}{\partial \rho}\left(\rho \frac{\partial}{\partial \rho}\right)+\frac{1}{\rho^{2}} \frac{\partial^{2}}{\partial \phi^{2}}+\frac{p_{z}}{p_{s}} \frac{\partial^{2}}{\partial z^{2}}+\omega^{2} p_{z} \tilde{p}_{s}\right] \psi=0
$$


where $\psi=\left\{E_{z}, H_{z}\right\}, p=\{\epsilon, \mu\}$ and $\tilde{p}=\{\mu, \epsilon\}$. On the grounds of the separation of variables method $[51,56,57]$, we can show that the elementary solution assumes the form

$$
R_{n}\left(\sqrt{\frac{p_{z}}{p_{s}}} k_{\rho} \rho\right) \Phi(n \phi) Z\left(k_{z} z\right)
$$

where $R_{n}(\cdot)$ is a solution to the Bessel differential equation of order $n$, and $\Phi(n \phi)$ and $Z\left(k_{z} z\right)$ are harmonic functions that satisfy the Laplace equation. The parameters $n, k_{\rho}$ and $k_{z}$ will be determined by the boundary conditions enforcement.

The general solution for $\psi$ may be written as a linear combination of the elementary solution [57], that is,

$$
\psi=\sum_{n} \sum_{k_{z}} C_{n, k_{z}} R_{n}\left(\sqrt{\frac{p_{z}}{p_{s}}} k_{\rho} \rho\right) \Phi(n \phi) Z\left(k_{z} z\right),
$$

where $C_{n, k_{z}}$ is a constant that will be determined by the boundary conditions enforcement. The separation constant $n$ assumes the discrete values $n=$ $\{0,1,2,3, \ldots\}$.

We will now consider the parcel of the fields associated with a wave propagating in the positive $z$-direction by selecting solutions to $R_{n}(\cdot), \Phi(n \phi)$ and $Z\left(k_{z} z\right)$ as

$$
\begin{aligned}
R_{n}\left(k_{\rho}^{e, h} \rho\right) & =a^{e, h} H_{n}^{(1)}\left(k_{\rho}^{e, h} \rho\right)+b^{e, h} J_{n}\left(k_{\rho}^{e, h} \rho\right), \\
\Phi(n \phi) & =e^{i n \phi}, \text { for } n=0, \pm 1, \pm 2, \ldots, \\
Z\left(k_{z} z\right) & =e^{i k_{z} z}
\end{aligned}
$$

where where $J_{n}(\cdot)$ and $H_{n}^{(1)}(\cdot)$ are first kind Bessel and Hankel functions of integer order $n, a^{e, h}$ and $b^{e, h}$ are modal amplitudes, and

$$
\sqrt{\frac{p_{z}}{p_{s}}} k_{\rho}=\alpha^{e, h} k_{\rho}=k_{\rho}^{e, h} .
$$

The superscripts $e, h$ indicate the correspondence for the axial electric and magnetic fields, respectively. Radial and longitudinal wavenumbers $k_{\rho}$ and $k_{z}$, respectively, are related by $k_{\rho}^{2}=k_{s}{ }^{2}-k_{z}^{2}$, with $k_{s}^{2}=\omega^{2} \mu_{s} \epsilon_{s}$.

\subsection{1}

\section{Axial Fields}

Adopting the compact matrix notation introduced in $[51,56]$, the longitudinal fields can be written as 


$$
\left[\begin{array}{c}
E_{z} \\
H_{z}
\end{array}\right]=\sum_{n=-\infty}^{\infty} \sum_{p=1}^{\infty}\left[\begin{array}{c}
e_{z, n p}(\rho) \\
h_{z, n p}(\rho)
\end{array}\right] e^{i n \phi} e^{i k_{z} z}
$$

where $p$ is related to $k_{z}$ and $k_{\rho}$. For simplicity, we will suppress the subscript $n p$, restoring it later as needed. The $\rho$-dependent parcel of the fields can be expressed as

$$
\left[\begin{array}{c}
e_{z}(\rho) \\
h_{z}(\rho)
\end{array}\right]=\overline{\bar{H}}_{z n}^{(1)}\left(k_{\rho} \rho\right) \bar{a}+\overline{\bar{J}}_{z n}\left(k_{\rho} \rho\right) \bar{b},
$$

where

$$
\overline{\bar{G}}_{z n}\left(k_{\rho} \rho\right)=\left[\begin{array}{cc}
G_{n}\left(k_{\rho}^{e} \rho\right) & 0 \\
0 & G_{n}\left(k_{\rho}^{h} \rho\right)
\end{array}\right],
$$

and $G_{n}=\left\{H_{n}^{(1)}, J_{n}\right\}$. The column-vectors $\bar{a}$ and $\bar{b}$ combine the modal amplitudes arranged as

$$
\bar{a}=\left[\begin{array}{l}
a_{n p}^{e} \\
a_{n p}^{h}
\end{array}\right] \quad \text { and } \quad \bar{b}=\left[\begin{array}{c}
b_{n p}^{e} \\
b_{n p}^{h}
\end{array}\right] .
$$

\subsection{2}

\section{Transversal Fields}

The transversal fields $E_{s}$ and $H_{s}$ can be expressed as a function of $E_{z}$ and $H_{z}$ counterparts [51]:

$$
\begin{aligned}
& \mathbf{E}_{s}=\frac{1}{k_{\rho}^{2}}\left[i k_{z} \boldsymbol{\nabla}_{s} E_{z}+i \omega \mu_{s} \boldsymbol{\nabla}_{s} \times\left(\hat{z} H_{z}\right)\right], \\
& \mathbf{H}_{s}=\frac{1}{k_{\rho}^{2}}\left[i k_{z} \boldsymbol{\nabla}_{s} H_{z}-i \omega \epsilon_{s} \boldsymbol{\nabla}_{s} \times\left(\hat{z} E_{z}\right)\right] .
\end{aligned}
$$

By suppressing the exponential harmonic functions, the $\rho$-dependent parcel of the fields can be written as

$$
\begin{aligned}
& {\left[\begin{array}{c}
e_{\phi, n p}(\rho) \\
h_{\phi, n p}(\rho)
\end{array}\right]=\overline{\bar{H}}_{\phi n}^{(1)}\left(k_{\rho} \rho\right) \bar{a}+\overline{\bar{J}}_{\phi n}\left(k_{\rho} \rho\right) \bar{b}} \\
& {\left[\begin{array}{c}
e_{\rho, n p}(\rho) \\
h_{\rho, n p}(\rho)
\end{array}\right]=\overline{\bar{H}}_{\rho n}^{(1)}\left(k_{\rho} \rho\right) \bar{a}+\overline{\bar{J}}_{\rho n}\left(k_{\rho} \rho\right) \bar{b}}
\end{aligned}
$$

where

$$
\begin{aligned}
& \overline{\bar{G}}_{\phi n}\left(k_{\rho} \rho\right)=\frac{1}{k_{\rho}^{2} \rho}\left[\begin{array}{cc}
-n k_{z} G_{n}\left(k_{\rho}^{e} \rho\right) & -i \omega \mu_{s} k_{\rho}^{h} \rho G_{n}^{\prime}\left(k_{\rho}^{h} \rho\right) \\
i \omega \epsilon_{s} k_{\rho}^{e} \rho G_{n}^{\prime}\left(k_{\rho}^{e} \rho\right) & -n k_{z} G_{n}\left(k_{\rho}^{h} \rho\right)
\end{array}\right], \\
& \overline{\bar{G}}_{\rho n}\left(k_{\rho} \rho\right)=\frac{1}{k_{\rho}^{2} \rho}\left[\begin{array}{cc}
i k_{z} k_{\rho}^{e} \rho G_{n}^{\prime}\left(k_{\rho}^{e} \rho\right) & -n \omega \mu_{s} G_{n}\left(k_{\rho}^{h} \rho\right) \\
n \omega \epsilon_{s} G_{n}\left(k_{\rho}^{e} \rho\right) & i k_{z} k_{\rho}^{h} \rho G_{n}^{\prime}\left(k_{\rho}^{h} \rho\right)
\end{array}\right] .
\end{aligned}
$$

In the above, $G_{n}^{\prime}(\cdot)$ is denoting the derivative of $G_{n}(\cdot)$ with respect to its 
argument.

\subsection{3}

\section{Circular and Coaxial Waveguides}

Consider now a circular waveguide with radius $\rho_{1}$ truncated by a perfect electric conductor (PEC) wall. The boundary conditions enforcement requires that tangential fields must vanish at $\rho=\rho_{1}$. Then, the fields $E_{\phi}$ and $E_{z}$ should be equal to zero in $\rho=\rho_{1}$. Also, the fields must be finite everywhere inside the waveguide. For this reason, the Hankel functions should not be used because its singular behavior at $\rho=0$.

Using (2-28) and (2-33), the transversal (to $\hat{\rho}$ ) fields at $\rho=\rho_{1}$ are given by

$$
\begin{aligned}
& e_{z}\left(\rho_{1}\right)=J_{n}\left(k_{\rho}^{e} \rho_{1}\right) b_{n p}^{e}, \\
& e_{\phi}\left(\rho_{1}\right)=\frac{1}{k_{\rho}^{2} \rho_{1}}\left[-n k_{z} J_{n}\left(k_{\rho}^{e} \rho_{1}\right) b_{n p}^{e}-i \omega \mu_{s} k_{\rho}^{h} \rho_{1} J_{n}^{\prime}\left(k_{\rho}^{h} \rho_{1}\right) b_{n p}^{h}\right] .
\end{aligned}
$$

From the above, we can obtain the following characteristic equations:

$$
\begin{array}{ll}
J_{n}\left(k_{\rho}^{e} \rho_{1}\right)=0, & \text { for } \mathrm{TM}^{z} \\
J_{n}^{\prime}\left(k_{\rho}^{h} \rho_{1}\right)=0, & \text { for } \mathrm{TE}^{z} .
\end{array}
$$

For a coaxial waveguide with inner radius $\rho_{0}$ and outer radius $\rho_{1}$, as depicted in Fig 2.2, the tangential fields $E_{\phi}$ and $E_{z}$ must be equal to zero at the radial-domain borders, i.e., at $\rho=\rho_{0}$ and $\rho=\rho_{1}$. For $\mathrm{TM}^{z}$ waves, the cited fields become

$$
\begin{aligned}
& e_{z}(\rho)=H_{n}^{(1)}\left(k_{\rho}^{e} \rho\right) a_{n p}^{e}+J_{n}\left(k_{\rho}^{e} \rho\right) b_{n p}^{e}, \\
& e_{\phi}(\rho)=\frac{-n k_{z}}{k_{\rho}^{2} \rho}\left[H_{n}^{(1)}\left(k_{\rho}^{e} \rho\right) a_{n p}^{e}+J_{n}\left(k_{\rho}^{e} \rho\right) b_{n p}^{e}\right] .
\end{aligned}
$$

At the boundaries, we obtain

$$
\begin{array}{ll}
H_{n}^{(1)}\left(k_{\rho}^{e} \rho_{0}\right) a_{n p}^{e}+J_{n}\left(k_{\rho}^{e} \rho_{0}\right) b_{n p}^{e}=0 & \text { in } \rho=\rho_{0}, \\
H_{n}^{(1)}\left(k_{\rho}^{e} \rho_{1}\right) a_{n p}^{e}+J_{n}\left(k_{\rho}^{e} \rho_{1}\right) b_{n p}^{e}=0 & \text { in } \rho=\rho_{1} .
\end{array}
$$

To fulfill the first condition in (2-43) we will consider

$$
a_{n p}^{e}=J_{n}\left(k_{\rho}^{e} \rho_{0}\right) \quad \text { and } \quad b_{n p}^{e}=-H_{n}^{(1)}\left(k_{\rho}^{e} \rho_{0}\right) .
$$

A similar procedure can be done for $\mathrm{TE}^{z}$ modes. This allows us to derive 

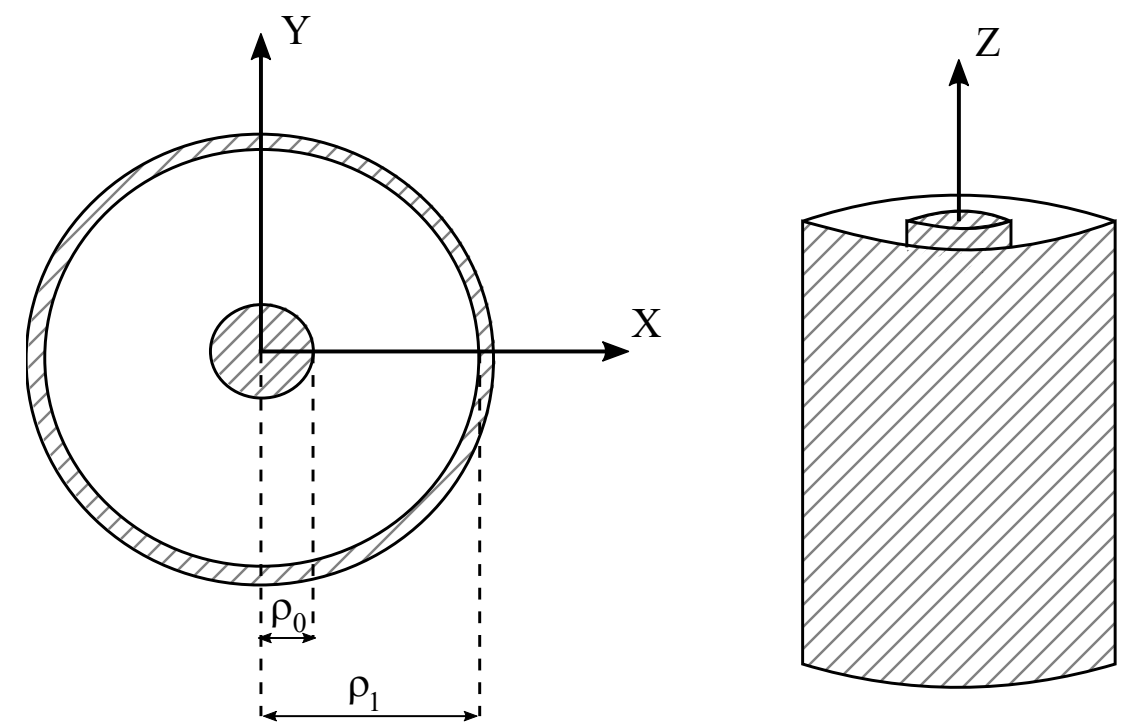

Figure 2.2: Coaxial waveguide. The shaded areas represent a perfect electric conductor (PEC) or a perfect magnetic conductor (PMC).

the following characteristic equations for a coaxial waveguide:

$$
\begin{array}{cc}
H_{n}^{(1)}\left(k_{\rho}^{e} \rho_{1}\right) J_{n}\left(k_{\rho}^{e} \rho_{0}\right)-J_{n}\left(k_{\rho}^{e} \rho_{1}\right) H_{n}^{(1)}\left(k_{\rho}^{e} \rho_{0}\right)=0, & \text { for } \mathrm{TM}^{z}, \\
H_{n}^{\prime(1)}\left(k_{\rho}^{h} \rho_{1}\right) J_{n}^{\prime}\left(k_{\rho}^{h} \rho_{0}\right)-J_{n}^{\prime}\left(k_{\rho}^{h} \rho_{1}\right) H_{n}^{\prime(1)}\left(k_{\rho}^{h} \rho_{0}\right)=0, & \text { for } \mathrm{TE}^{z} .
\end{array}
$$

Notice that fields can be decomposed into TE and TM parcels only when the waveguide is homogeneous. For a lossless media, the eigenvalues $k_{\rho}$ will be always real and they are numerically found, within a root-finding algorithm in the $k_{\rho}$ plane. With them, we determine the values of $k_{z}$ and the corresponding modal amplitudes.

\subsection{4}

\section{Root-finding in the $k_{\rho}$ plane}

For the homogeneous case, the characteristic equation for $\mathrm{TM}^{z}$ modes is given by (2-39) and (2-46) for circular and coaxial waveguides, respectively. The values of $k_{\rho}$ that satisfies them are the zeros of such function, and each one will contribute to a modal field.

We will start defining points in the $k_{z}$ complex plane that will delimit the maximum value of $k_{z}$ in the form

$$
k_{z, \max }=x+i y .
$$

Considering first a lossless media, we predict that the values of $k_{z}$ will be exclusively pure real or pure imaginary numbers. From the separation equation $k_{s}^{2}=k_{z}^{2}+k_{\rho}^{2}$, the maximum value in the real $k_{z}$ axis will be limited by $x=\operatorname{Re}\left\{k_{s}\right\} \times 1.01$, where the multiplication factor 1.01 were employed to 
guarantee that all roots will be included in the search region. The maximum imaginary part can be approximated by the relation

$$
y=\operatorname{Im}\left\{k_{z}\right\}=\frac{m \pi}{\rho_{1}} \times 1.01,
$$

where $m$ is an estimated number of modes and $\rho_{1}$ is the outermost radius that truncates the waveguide.

For a lossy media, the limiting value of $x$ will remain the same, but the maximum point in the imaginary axis should be corrected. That can be done based on a value of attenuation in decibel $\left(A_{d B}\right)$ that the wave will suffer and in an axial distance $(\Delta z)$ that the associated wave will travel. Namely:

$$
y=-\frac{\ln (10) \times A_{d B}}{20 \times \Delta z \times 1.01} .
$$

Knowing an approximated value for $k_{z, \max }$, it is possible to define the maximum counterpart in the $k_{\rho}$-plane using

$$
k_{\rho, \max }=\sqrt{k_{s}^{2}-k_{z, \max }^{2}} .
$$

The region of search will be delimited from $-x$ to $x$ in the real axis and from 0 to $y$ in the imaginary one, as depicted in Fig 2.3.

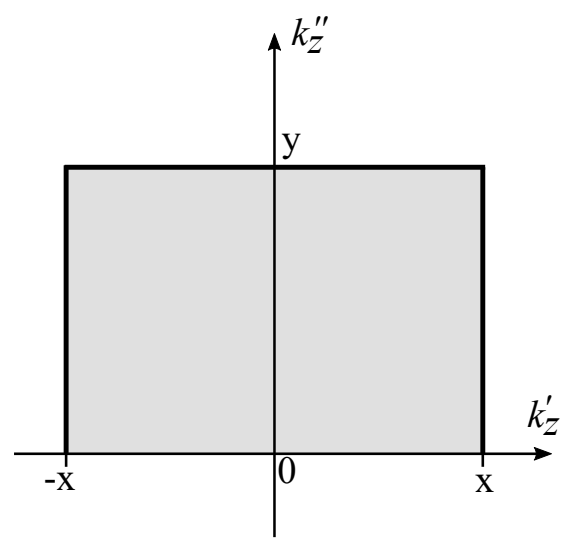

Figure 2.3: Region of search on the $k_{z}$-complex plane, where $k_{z}^{\prime}$ is the real and $k_{z}^{\prime \prime}$ is the imaginary part of $k_{z}$, respectively.

The zeros of the Bessel function are proportional to $p \pi$ as its arguments is large, with $p=\{1,2, \ldots\}$. Therefore, we can estimate that the number of modes will be

$$
N_{\text {modes }}=\frac{\operatorname{Re}\left\{k_{\rho, \max }\right\} \times p}{\pi}+2,
$$

where the factor two was added to guarantee that all the desired roots are included. For the circular waveguide case, the bisection method is used to find the roots of the characteristic equation (2-39). The modal amplitudes are then defined as 


$$
a^{e, h}=0 \quad \text { and } \quad b^{e, h}=1 .
$$

For the coaxial waveguide, a different approach has to be used. To avoid numerical issues, it is necessary to make two considerations. First, instead of use the product $J_{n}(\cdot) H_{n}^{(1)}(\cdot)$, we will use the first and second Hankel functions, $H_{n}^{(1)}(\cdot)$ and $H_{n}^{(2)}(\cdot)$, since every pair of Bessel functions shares the same set of eigenvalues for the problem at hand. Then, for $\mathrm{TM}^{z}$ fields, we can rewrite the characteristic equation (2-46) as

$$
H_{n}^{(1)}\left(k_{\rho}^{e} \rho_{1}\right) H_{n}^{(2)}\left(k_{\rho}^{e} \rho_{0}\right)-H_{n}^{(2)}\left(k_{\rho}^{e} \rho_{1}\right) H_{n}^{(1)}\left(k_{\rho}^{e} \rho_{0}\right)=0 .
$$

Secondly, we will introduce conditioned functions, also to avoid numerical issues. The scaled Hankel functions, for example, can be defined as

$$
\begin{aligned}
& H_{n}^{(1)}(x)=\hat{H}_{n}^{(1)}(x) e^{+i x} \\
& H_{n}^{(2)}(x)=\hat{H}_{n}^{(2)}(x) e^{-i x} .
\end{aligned}
$$

In addition, using a similar approach as in [58], the inner and outer radii can be written in terms of the scale factor $\left(\lambda=\rho_{1} / \rho_{0}\right)$. Rewriting the arguments of Hankel function, we obtain

$$
k_{\rho}^{e} \rho_{0}=y \quad \text { and } \quad k_{\rho}^{e} \rho_{1}=\lambda y .
$$

The characteristic equation is now in the form

$$
\hat{H}_{n}^{(1)}(\lambda y) e^{+i \lambda y} \hat{H}_{n}^{(2)}(y) e^{-i y}-\hat{H}_{n}^{(2)}(\lambda y) e^{-i \lambda y} \hat{H}_{n}^{(1)}(y) e^{+i y}=0,
$$

and after a few manipulations, we obtain

$$
\hat{H}_{n}^{(2)}(y) \hat{H}_{n}^{(1)}(\lambda y) e^{2 i y(\lambda-1)}-\hat{H}_{n}^{(1)}(y) \hat{H}_{n}^{(2)}(\lambda y)=0,
$$

which is the characteristic equation of the coaxial waveguide to be solved. The values of $y$ may be complex even though $k_{\rho}$ is real, due to the media losses. In this case, the bisection method does not guarantee that all complexvalues roots are obtained. By that reason, the Muller method is used instead. The same considerations about the region of search of the circular waveguide problem were used here.

Finally, after solving $y$, it is possible to convert them to $k_{\rho}$ and define the modal amplitudes as

$$
\begin{array}{ll}
a^{e}=J_{n}\left(k_{\rho}^{e} \rho_{0}\right), & b^{e}=-H_{n}^{(1)}\left(k_{\rho}^{e} \rho_{0}\right), \\
a^{h}=J_{n}^{\prime}\left(k_{\rho}^{h} \rho_{0}\right), & b^{h}=-H_{n}^{\prime(1)}\left(k_{\rho}^{h} \rho_{0}\right) .
\end{array}
$$

In a homogeneous waveguide, TE and TM modes are decoupled, and we have different characteristic equations for each mode. As will be seen in the 
next section, this does not occur for inhomogeneous media, where the field solutions are a hybrid combination of TE and TM parcels.

The coaxial homogeneous waveguide can also include the TEM mode when the order $n$ is zero. In this scenario, $k_{\rho}=0$ is a solution and the modal amplitudes will all be null, except for $a^{e}$, which will be equal to one. Despite that, the fields non-null $E_{\rho}$ and $H_{\phi}$ may exist.

\section{2}

\section{Inhomogeneous Waveguide}

Consider a waveguide with $N$ radial layers and defined by the radii $\left\{\rho_{0}, \rho_{1}, \ldots, \rho_{N}\right\}$, as shown in Fig. 2.4. By omitting the $n p$ subscript, the $\rho$ dependent parcel of the fields in the layer $j$ can be written in the form

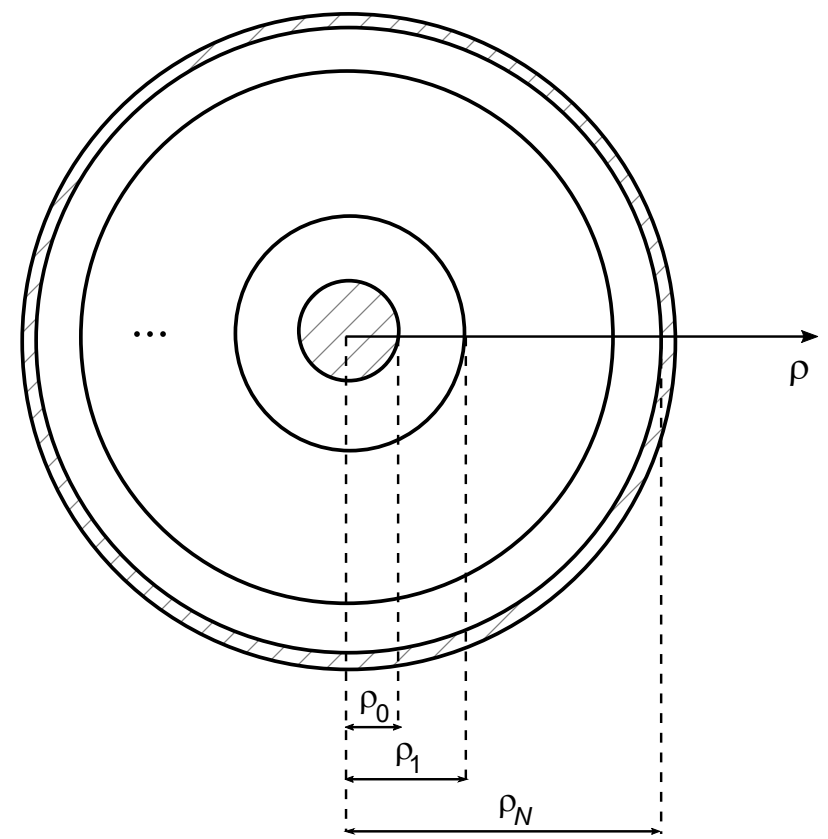

Figure 2.4: Inhomogeneous coaxial waveguide with $N$ different layers.

$$
\left[\begin{array}{c}
e_{j \alpha}(\rho) \\
h_{j \alpha}(\rho)
\end{array}\right]=\overline{\bar{H}}_{\alpha n}^{(1)}\left(k_{j \rho} \rho\right) \bar{a}_{j}+\overline{\bar{J}}_{\alpha n}\left(k_{j \rho} \rho\right) \bar{b}_{j},
$$

where $\alpha=\{\rho, \phi, z\}$ and $j$ is the waveguide layer, with $j=\{1,2, \ldots N\}$. The $e_{j \alpha}(\rho)$ and $h_{j \alpha}(\rho)$ functions are the only one that vary along the radial layers. By enforcing the continuity of the $z$ and $\phi$ field components at the interfaces between layer $j$ and its adjacent, we can obtain

$$
\begin{aligned}
& {\left[\begin{array}{l}
e_{j \alpha}(\rho) \\
h_{j \alpha}(\rho)
\end{array}\right]=\left[\overline{\bar{H}}_{\alpha n}^{(1)}\left(k_{j \rho} \rho\right)+\overline{\bar{J}}_{\alpha n}\left(k_{j \rho} \rho\right) \tilde{\overline{\bar{R}}}_{j, j+1}^{(\rho)}\right] \bar{a}_{j}, \text { or }} \\
& {\left[\begin{array}{l}
e_{j \alpha}(\rho) \\
h_{j \alpha}(\rho)
\end{array}\right]=\left[\overline{\bar{H}}_{\alpha n}^{(1)}\left(k_{j \rho} \rho\right) \tilde{\overline{\bar{R}}}_{j, j-1}^{(\rho)}+\overline{\bar{J}}_{\alpha n}\left(k_{j \rho} \rho\right)\right] \bar{b}_{j},}
\end{aligned}
$$


In the above, (2-62) is relating a wave propagating to $+\hat{\rho}$ that experienced a reflection, and (2-63) propagating in the opposite direction, i.e., $-\hat{\rho}$.

The generalized reflection matrix $\tilde{\overline{\bar{R}}}_{j, j+1}^{(\rho)}$ is a 2 -by-2 matrix given by [51, $56]$

$$
\tilde{\overline{\bar{R}}}_{j, j \pm 1}^{(\rho)}=\overline{\bar{R}}_{j, j \pm 1}^{(\rho)}+\overline{\bar{T}}_{j \pm 1, j}^{(\rho)} \tilde{\overline{\bar{R}}}_{j \pm 1, j \pm 2}^{(\rho)}\left(\overline{\bar{I}}-\tilde{\overline{\bar{R}}}_{j \pm 1, j}^{(\rho)} \tilde{\overline{\bar{R}}}_{j \pm 1, j \pm 2}^{(\rho)}\right)^{-1} \overline{\bar{T}}_{j, j+1}^{(\rho)}
$$

where $\overline{\bar{I}}$ is the identity matrix. The local transmission and reflection matrices are defined according to

$$
\begin{aligned}
& \overline{\bar{R}}_{j, j+1}^{(\rho)}=\bar{D}_{j a}^{-1}\left[\overline{\bar{H}}_{\phi j+1, j} \overline{\bar{H}}_{z j, j}-\overline{\bar{H}}_{\phi j+1, j} \overline{\bar{H}}_{z j+1, j} \overline{\bar{H}}_{\phi j+1, j}^{-1} \overline{\bar{H}}_{\phi j, j}\right] \\
& \overline{\bar{R}}_{j+1, j}^{(\rho)}=\bar{D}_{j b}^{-1}\left[\overline{\bar{J}}_{\phi j, j} \overline{\bar{J}}_{z j, j} \overline{\bar{J}}_{\phi j, j}^{-1}-\overline{\bar{J}}_{\phi j, j} \overline{\bar{J}}_{z j+1, j}\right] \\
& \overline{\bar{T}}_{j, j+1}^{(\rho)}=\bar{D}_{j b}^{-1}\left[\overline{\bar{J}}_{\phi j, j} \overline{\bar{H}}_{z j, j}-\overline{\bar{J}}_{\phi j, j} \overline{\bar{J}}_{z j, j} \overline{\bar{J}}_{\phi j, j}^{-1} \overline{\bar{H}}_{\phi j, j}\right] \\
& \overline{\bar{T}}_{j+1, j}^{(\rho)}=\bar{D}_{j a}^{-1}\left[\overline{\bar{H}}_{\phi j+1, j} \overline{\bar{H}}_{z j+1, j} \overline{\bar{H}}_{\phi j+1, j} \overline{\bar{J}}_{\phi j+1, j}-\overline{\bar{H}}_{\phi j+1, j} \overline{\bar{J}}_{z j+1, j}\right] \\
& \bar{D}_{j a}=\overline{\bar{H}}_{\phi j+1, j} \overline{\bar{H}}_{z j+1, j} \overline{\bar{H}}_{\phi j+1, j}^{-1} \overline{\bar{J}}_{\phi j, j}-\overline{\bar{H}}_{\phi j+1, j} \overline{\bar{J}}_{z j, j} \\
& \bar{D}_{j b}=\overline{\bar{J}}_{\phi j, j} \overline{\bar{H}}_{z j+1, j}-\overline{\bar{J}}_{\phi j, j} \overline{\bar{J}}_{z j, j} \overline{\bar{J}}_{\phi j, j}^{-1} \overline{\bar{H}}_{\phi j+1, j}
\end{aligned}
$$

where the following compact notation was adopted:

$$
\overline{\bar{H}}_{\alpha i, j}=\overline{\bar{H}}_{\alpha n}\left(k_{i \rho} \rho_{j}\right) \quad \text { and } \quad \overline{\bar{J}}_{\alpha i, j}=\overline{\bar{J}}_{\alpha n}\left(k_{i \rho} \rho_{j}\right)
$$

with $\alpha=\{\phi, z\}$. From (2-62) and (2-63), we have the following relation between the vector amplitudes:

$$
\bar{a}_{j}=\tilde{\overline{\bar{R}}}_{j, j-1}^{(\rho)} \bar{b}_{j} \quad \text { and } \quad \bar{b}_{j}=\tilde{\overline{\bar{R}}}_{j, j+1}^{(\rho)} \bar{a}_{j} .
$$

Replacing $\bar{a}_{j}$ in the $\bar{b}_{j}$ expression, we obtain

$$
\begin{aligned}
& \bar{b}_{j}=\tilde{\overline{\bar{R}}}_{j, j+1}^{(\rho)} \tilde{\overline{\bar{R}}}_{j, j-1}^{(\rho)} \bar{b}_{j} \\
& {\left[\overline{\bar{I}}-\tilde{\overline{\bar{R}}}_{j, j+1}^{(\rho)} \tilde{\overline{\bar{R}}}_{j, j-1}^{(\rho)}\right] \bar{b}_{j}=\overline{0} .}
\end{aligned}
$$

Then, the non-trivial solution is given by

$$
\operatorname{det}\left(\overline{\bar{I}}-\tilde{\overline{\bar{R}}}_{j, j+1}^{(\rho)} \tilde{\overline{\bar{R}}}_{j, j-1}^{(\rho)}\right)=0 .
$$

Therefore, any $k_{z}$ that satisfy the characteristic equation (2-74) is a proper solution the problem at hand. By selecting $j=N$, we obtain

$$
f\left(k_{z}\right)=\operatorname{det}\left(\overline{\bar{I}}-\overline{\bar{R}}_{N, N+1}^{(\rho)} \tilde{\overline{\bar{R}}}_{N, N-1}^{(\rho)}\right)=0 .
$$

Notice that when $j=N$, we have $\tilde{\overline{\bar{R}}}_{N, N+1}^{(\rho)}=\overline{\bar{R}}_{N, N+1}^{(\rho)}$. The values of $k_{z}$ will be the same, independent of the layer. In order to find the eigenvalue 
solutions in (2-75), it is appropriated to remove the singularities of this characteristic equation. By using a procedure similar to that in [51, 59], a pole-free characteristic equation can be obtained:

$$
f_{p f}\left(k_{z}\right)=\prod_{j=1}^{j=N+1} \operatorname{det}\left[\left(k_{j}^{2}-k_{z}^{2}\right)^{m(j) / 2} \overline{\bar{D}}_{j-1}\left(\overline{\bar{I}}-\overline{\bar{R}}_{j-1, j}^{(\rho)} \tilde{\overline{\bar{R}}}_{j-1, j-2}^{(\rho)}\right)\right]
$$

where

$$
\overline{\bar{D}}_{j}= \begin{cases}\overline{\bar{D}}_{j a}, & \text { if } j=N \\ \overline{\bar{D}}_{j b}, & \text { if } j<N\end{cases}
$$

and

$$
m(j)= \begin{cases}\frac{3}{2}-\delta_{1, N}\left[\frac{1}{2}+\delta_{0, n}-\frac{u(0)}{2}\right] & \\ -\delta_{0, \rho_{0}}\left[\frac{1}{2}+n+\delta_{0, n}\left(1-\delta_{1, N}\right)\right]+\frac{u(0)}{2}, & \text { if } j=1 \\ \frac{3}{2}+\frac{u(N)}{2}, & \text { if } j=N \text { and } N>1 \\ 0, & \text { if } j=N+1 \\ 2, \text { otherwise. } & \end{cases}
$$

In the above, $\delta_{i, j}$ is the Kronecker delta (which is 1 if $i=j$ and zero otherwise), and the function $u(j)$ indicates an impedance boundary condition for a truncating the waveguide at $\rho_{j}$. In case of perfect electric conductor (PEC) or perfect magnetic conductor (PMC), we have $u(j)=0$. For the impedance wall case, boundaries conditions used to solve the characteristic equation are slightly different from those showed here. Further details can be found in [51].

\subsection{1}

\section{Root-finding in the $k_{z}$ plane}

The majority of propagation problems in inhomogeneous, anisotropic, ans lossy media requires to find the complex-valued roots of the characteristic equations. However, they cannot be found analytically and root-finding algorithms are used to solve this drawback.

The eigenvalues that are inside a region of interest in the complex plane can be found by several numerical methods, for example, the Muller method, 2D bisection method [50], the Cauchy method [28, 49], among others. In [60], a global algorithm was developed to accomplish that. Here, the Cauchy's Argument Principle [61], also known as the winding number, will be employed to calculate the number of zeros of our characteristic function. The winding number of a complex function in a closed contour $C$ is given by [51, 59, 62-64]

$$
N_{0}-N_{p}=\frac{1}{2 \pi i} \oint_{C} \frac{f_{p f}^{\prime}\left(k_{z}\right)}{f_{p f}\left(k_{z}\right)} d k_{z}
$$

where $N_{p}$ is the number of poles and $N_{0}$ the number of zeros. As we have a 
pole-free characteristic equation, the above integral will gives us the number of zeros $N_{0}$. We exploited here four different methods for calculating $N_{0}$. The first method is a direct application of equation (2-79) in which the region of search is defined in the same manner as in the homogeneous case, with a limiting contour oriented in the Cauchy's counterclockwise sense. As mentioned in [65], it is convenient due to numerical reasons to divide the contour into segments.

The second method is the one applied in [51], where the region of search is depicted in Fig 2.5. The contour integral $C$ is split and reduced into three line integrals on over $C_{1}, C_{2}$ and $C_{3}$ (please, see [51] for further details). Then (2-79) reduces to

$$
N_{0}=\frac{1}{2 \pi i}\left[\int_{C_{1}} \frac{f_{p f}^{\prime}\left(k_{z}\right)}{f_{p f}\left(k_{z}\right)} d k_{z}+\int_{C_{2}} \frac{f_{p f}^{\prime}\left(k_{z}\right)}{f_{p f}\left(k_{z}\right)} d k_{z}+\int_{C_{3}} \frac{f_{p f}^{\prime}\left(k_{z}\right)}{f_{p f}\left(k_{z}\right)} d k_{z}\right] .
$$

The function $f_{p f}\left(k_{z}\right)$ is a product of functions, as seen in (2-76), and then, it is possible to consider writing it as

$$
f_{p f}\left(k_{z}\right)=\prod_{j=1}^{j=N+1} f_{j}
$$

where

$$
f_{j}=\operatorname{det}\left[\left(k_{j}^{2}-k_{z}^{2}\right)^{m(j) / 2} \overline{\bar{D}}_{j-1}\left(\overline{\bar{I}}-\overline{\bar{R}}_{j-1, j}^{(\rho)} \tilde{\overline{\bar{R}}}_{j-1, j-2}^{(\rho)}\right)\right] .
$$

The derivative of this product might be expanded as a sum of derivatives, using the logarithmic differentiation [66]. This method is usually employed in cases where the function is composed by a product of other functions. The logarithmic will then transform the original function in a sum of separate parts, rendering this approach a clever to compute derivatives.

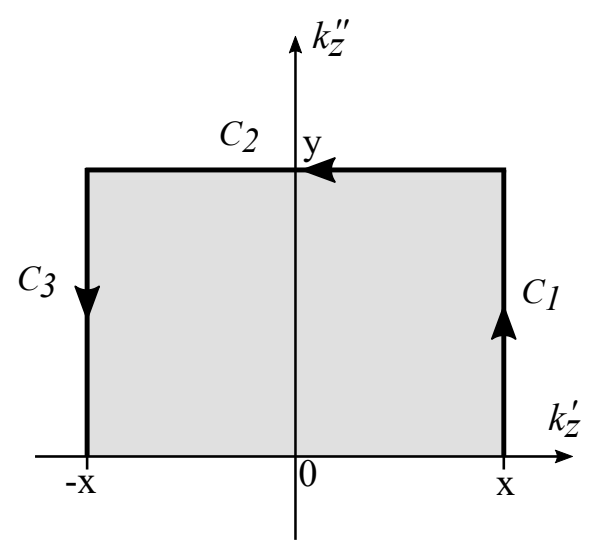

Figure 2.5: Region of search on the $k_{z}$-complex plane, and the contours $C_{1}, C_{2}$ and $C_{3}$ used in the calculation of the winding number. 
By taking the logarithmic on both sides of (2-81), we obtain [67]

$$
\begin{aligned}
\ln \left(f_{p f}\left(k_{z}\right)\right) & =\ln \left(\prod_{j=1}^{j=N+1} f_{j}\right) \\
& =\sum_{j=1}^{j=N+1} \ln \left(f_{j}\right)
\end{aligned}
$$

and, by taking then the derivative, we can obtain

$$
\frac{f_{p f}^{\prime}\left(k_{z}\right)}{f_{p f}\left(k_{z}\right)}=\sum_{j=1}^{j=N+1} \frac{f_{j}^{\prime}}{f_{j}} .
$$

The third method is then given by a combination of the above results with the initial equation (2-79) transformed into

$$
N_{0}=\frac{1}{2 \pi i} \sum_{j=1}^{j=N+1} \oint_{C} \frac{f_{j}^{\prime}\left(k_{z}\right)}{f_{j}\left(k_{z}\right)} d k_{z} .
$$

Finally, by combining (2-85) and (2-80), we can formulate our fourth method, namely:

$$
N_{0}=\frac{1}{2 \pi i} \sum_{j=1}^{j=N+1} \sum_{q=1}^{3} \oint_{C_{q}} \frac{f_{j}^{\prime}\left(k_{z}\right)}{f_{j}\left(k_{z}\right)} d k_{z} .
$$

It should be observed that the format above is a parallel-friendly version of the original winding number defined in (2-79). In addition, the derivative $f_{j}^{\prime}\left(k_{z}\right)$ can be calculated analytically in view of (2-82).

After finding $N_{0}$, a root finding algorithm based on Muller's method can be used to determine the eigenvalues $k_{z}$ of our characteristic equation. Having the values of $k_{z}$, it is then possible to find the modal amplitudes $\bar{a}_{j}$ and $\bar{b}_{j}$ for all layers. Consequently, we have all ingredients for computing the electromagnetic field solution.

The modal amplitude $\bar{b}_{j}$ was defined in $[51,63]$ as

$$
\bar{b}_{j-1}=\left(\overline{\bar{I}}-\overline{\bar{R}}_{j-1, j}^{(\rho)} \tilde{\overline{\bar{R}}}_{j-1, j-2}^{(\rho)}\right)^{-1} \overline{\bar{T}}_{j, j-1}^{(\rho)} \bar{b}_{j} .
$$

The value of $\bar{b}_{j}$ in the last layer is the null space of matrix $\overline{\bar{M}}_{N}$, which is given by

$$
\begin{gathered}
\overline{\bar{M}}_{N}=\left(\overline{\bar{I}}-\overline{\bar{R}}_{N, N+1}^{(\rho)} \tilde{\overline{\bar{R}}}_{N, N-1}^{(\rho)}\right), \\
\bar{b}_{N}=\operatorname{null}\left(\overline{\bar{M}}_{N}\right)
\end{gathered}
$$

The amplitudes $\bar{b}_{j}$ for the remaining layers $j=\{1,2, \ldots N-1\}$ can then be obtained recursively using $(2-88), \bar{a}_{j}$, and (2-72). 


\section{3}

\section{Validation}

In this section, we present simulation results obtained from a numerical algorithm implemented in the Matlab platform [68]. A series of representative examples of waveguide scenarios will be evaluated in order to validate or mathematical formulation and the corresponding numerical algorithm.

\section{Comparison between the Winding Number Methods}

Before introducing the main simulations, we will expose the difference found among the four methods used to calculate the number of zeros inside a waveguide discussed in Section 2.2.1. It was considered a two layers coaxial waveguide with parameters listed in Table 2.1. Simulations were performed at $10 \mathrm{GHz}$.

The number of zeros found in each mode can be seen in Table 2.2. As we employed a numerical differentiation, the results obtained can be approximated to a real integer, as expected. We have employed the adaptive integration function integral $(\cdot)$ provided in Matlab [68] using the relative and absolute errors of $10^{-8}$ and $10^{-13}$, respectively. The region of search was delimited by $x=-368.15$ and $x=368.15$ in the real axis, and by $y=0$ and $y=6346$ in the imaginary one. As it was a lossless media, we used equation (2-49) to determine the value of $y$, considering $m$ as 10 . The values are close to each other and the major difference is in the imaginary part when using Methods 3 and 4. Despite that, all of the winding numbers approach are able to effectively found 13 eigenvalues.

Table 2.3 shows a comparative between computational time spent in each method. Some simulations were performed and the average time was considered in this comparison. We observe that Methods 1 and 2 are faster and Methods 3 and 4 are slower, showing that replace the product of functions to a sum increased the time spent on this operation. Even though Method 4 has the highest computational time, it is possible to parallelize its integral calculations, specially for waveguides with a large number of radial layers. And

\begin{tabular}{cccc}
\hline Parameters & \multicolumn{3}{c}{ Layers } \\
\hline$\rho(\mathrm{mm})$ & 1.84 & 2 & 5 \\
$\epsilon_{r}$ & 2.55 & 1 & - \\
\hline \multicolumn{4}{c}{$\sigma=10^{-6}$} \\
\hline
\end{tabular}

Table 2.1: Dimensions and parameters of the reference waveguide. Other constitutive parameters are equal to those of vacuum. 
as said before, the derivative $f_{j}^{\prime}\left(k_{z}\right)$ can be calculated analytically in view of (2-82); this was done here, but is a important investigation topic for future research.

To explore the fields behavior, some waveguides were selected to be analyzed in the following sections.

\section{Waveguide 1 - A four-layer coaxial waveguide}

The first waveguide analyzed is a four-layer coaxial waveguide, radially truncated by PEC, with the parameters listed in Table 2.4. The electrical conductivity in all of the layers is very small, with $\sigma=10^{-6} \mathrm{~S} / \mathrm{m}$. This will result in an almost lossless media. The first nine $k_{z}$ for the frequencies of 20 , 40, 60 and $80 \mathrm{GHz}$ are presented in Fig 2.6. Fig 2.7 shows the normalized field components $E_{z}$ and $E_{\rho}$ for the first propagating mode (defined by the one with the smallest $\left.\operatorname{Im}\left(k_{z}\right)\right)$. We are presenting fields associated with the azimuthal index $n=0$.

As expected, the $E_{z}$ field vanishes at the PEC. Even changing its behavior as it crosses the layers, this field component is continuous along the radial direction. The $\rho$-component (normal to the radial interfaces) of the electric field, however, is discontinuous at the interfaces at 3, 5, and $10 \mathrm{~mm}$.

Fig. 2.8 presents the fields for the first three TE $\left(E_{z}\right)$ and TM $\left(E_{\phi}\right)$ modes for the waveguide 1 for the same operating frequencies used above. Again, we can observe that our field solutions fulfill all the boundary conditions, i.e., the tangential fields are continuous for all values of $\rho$, and vanish at the PEC.

It is important to observe that, as the frequency increases, the $E_{\rho}$ discontinuities becomes smaller, as noted in Fig 2.7. It is because the surface charge density $\varrho_{s}$ at the interface between layer become vanishing as the wavelength decreases. For supporting such point, consider the results shown in Fig 2.9, for the same field but at $160 \mathrm{GHz}$ : at $\rho=5 \mathrm{~mm}$ the $E_{\rho}$ field component is virtually continuous. At at $\rho=10 \mathrm{~mm}$, due to the relatively more large permittivity contrast of the layers (see Table 2.5) the step discontinuous is more prominent.

\begin{tabular}{ccc}
\hline Method & Reference Equation & $N_{0}$ \\
\hline 1 & $(2-79)$ & $12.999999999996220+0.000001429609161 i$ \\
2 & $(2-80)$ & $12.999999999899739+0.000001429416197 i$ \\
3 & $(2-86)$ & $12.999999999581870+0.000012527010247 i$ \\
4 & $(2-87)$ & $12.999999999927436+0.000012527046568 i$ \\
\hline
\end{tabular}

Table 2.2: Number of zeros of reference waveguide for the different methods presented. 


\begin{tabular}{cc}
\hline Method & Average time $(\mathrm{s})$ \\
\hline 1 & 3.8517624 \\
2 & 3.6335266 \\
3 & 6.2435971 \\
4 & 6.9485663 \\
\hline
\end{tabular}

Table 2.3: Average time spent on each method.

\begin{tabular}{cccccc}
\hline Parameters & \multicolumn{5}{c}{ Layers } \\
\hline$\epsilon_{r}$ & 2.5 & 3 & 3.5 & 1 & - \\
$\rho(\mathrm{mm})$ & 1 & 3 & 5 & 10 & 15 \\
\hline \multicolumn{5}{c}{$\sigma=10^{-6}(\mathrm{~S} / \mathrm{m})$} \\
\hline
\end{tabular}

Table 2.4: Dimensions and parameters of waveguide 1. Other constitutive parameters are equal to those of vacuum.

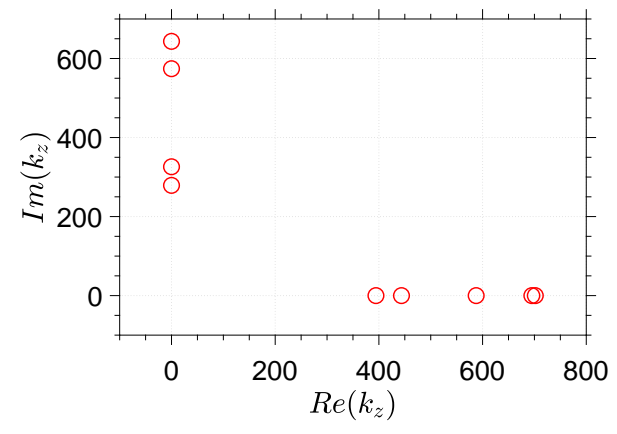

2.6(a): $20 \mathrm{GHz}$

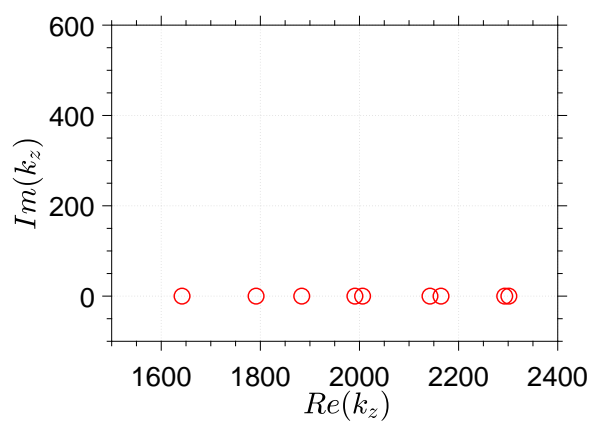

2.6(c): $60 \mathrm{GHz}$

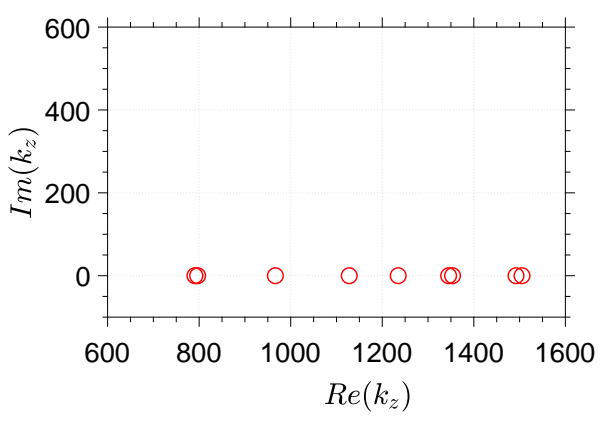

2.6(b): $40 \mathrm{GHz}$

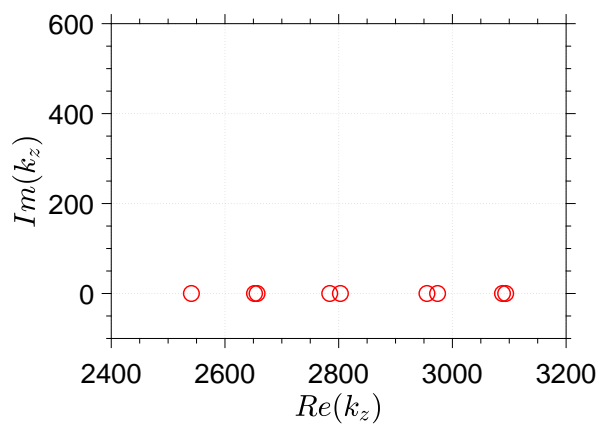

2.6(d): $80 \mathrm{GHz}$

Figure 2.6: First nine wavenumbers $k_{z}$ for waveguide 1 at different operating frequencies. 


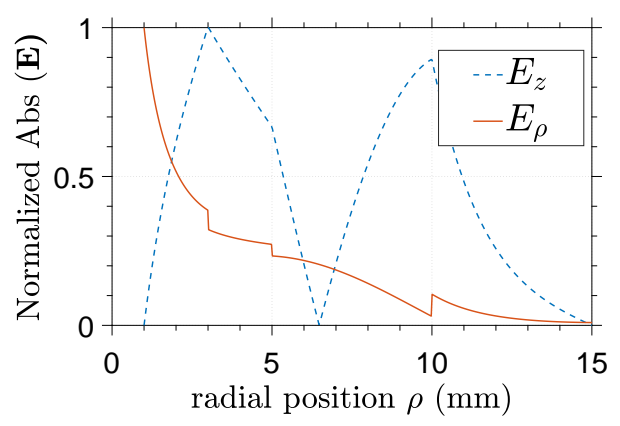

$2.7(\mathrm{a}): 20 \mathrm{GHz}$

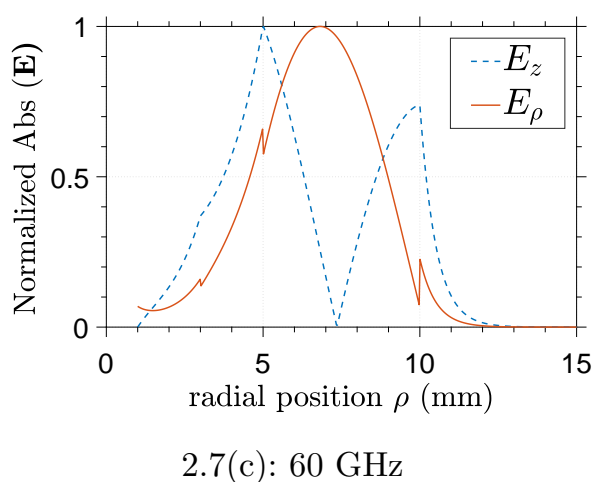

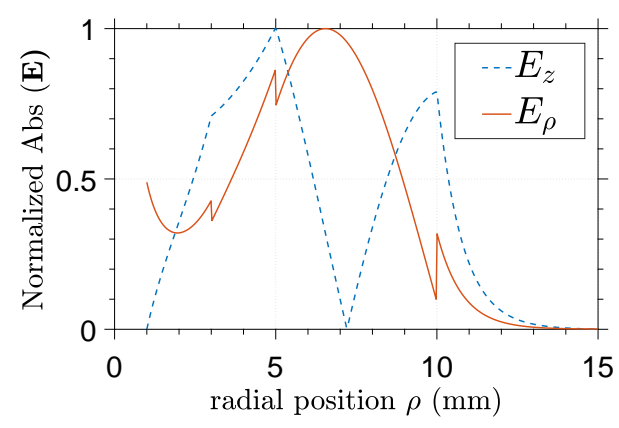

$2.7(\mathrm{~b}): 40 \mathrm{GHz}$

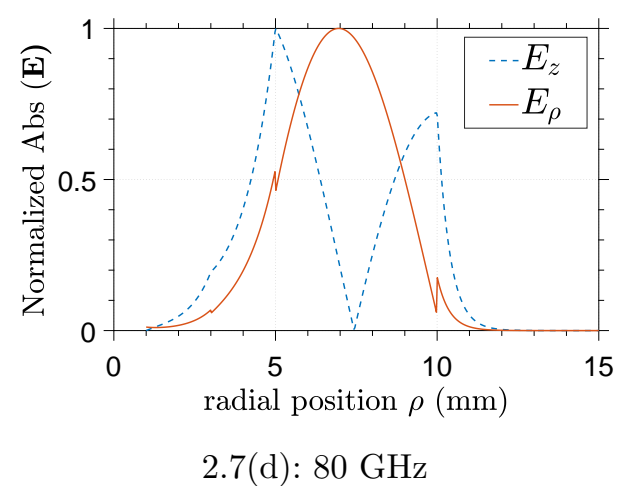

Figure 2.7: Normalized $E_{z}$ and $E_{\rho}$ fields of the first propagating mode in waveguide 1 at different operating frequencies. 


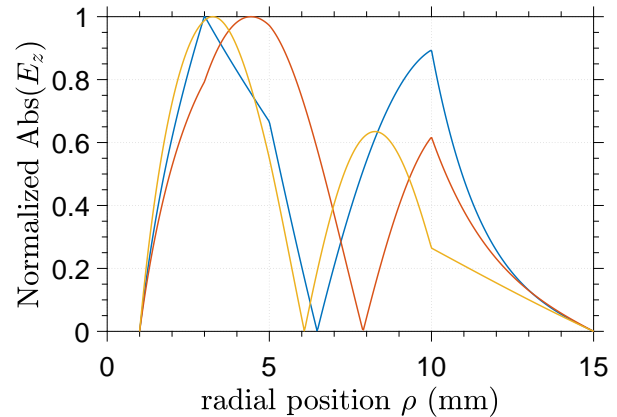

2.8(a): First three propagating modes of $E_{z}$ at $20 \mathrm{GHz}$

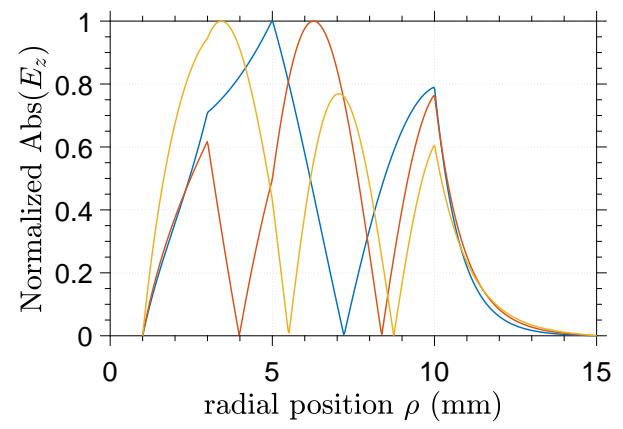

2.8(c): First three propagating modes of $E_{z}$ at $40 \mathrm{GHz}$

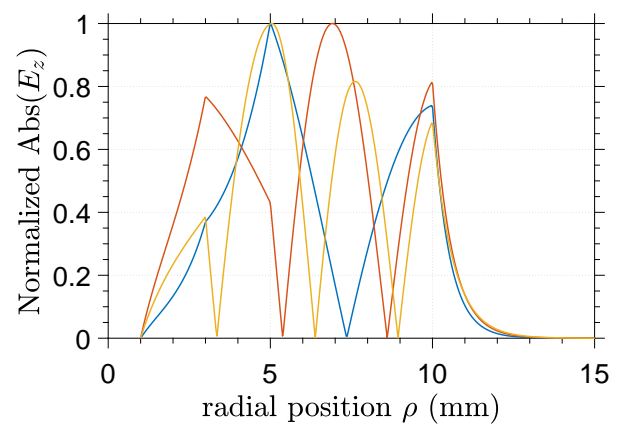

2.8(e): First three propagating modes of $E_{z}$ at $60 \mathrm{GHz}$

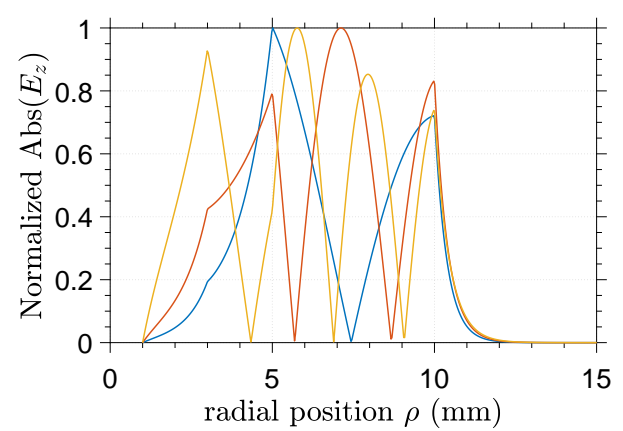

2.8(g): First three propagating modes of $E_{z}$ at $80 \mathrm{GHz}$

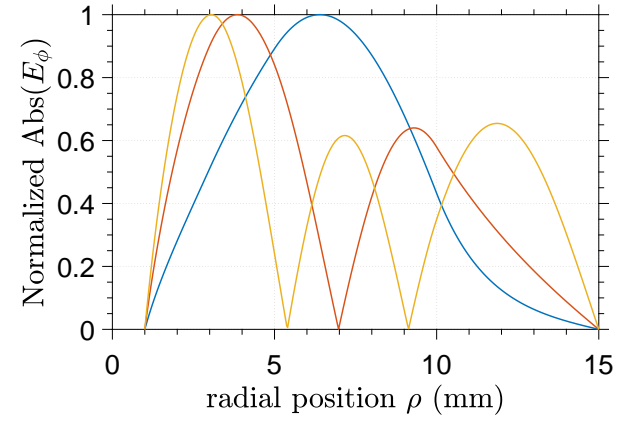

2.8(b): First three propagating modes of $E_{\phi}$ in $20 \mathrm{GHz}$

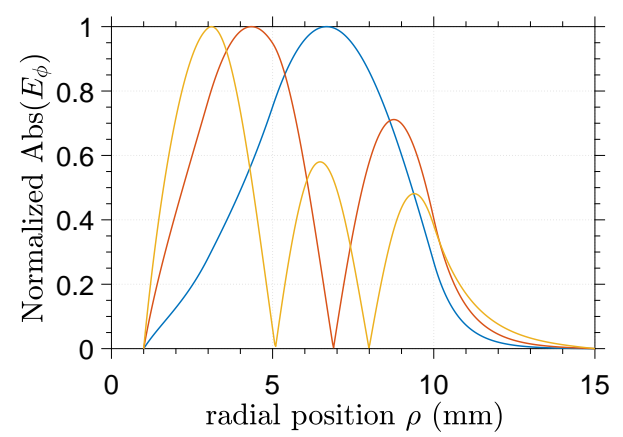

2.8(d): First three propagating modes of $E_{\phi}$ in $40 \mathrm{GHz}$

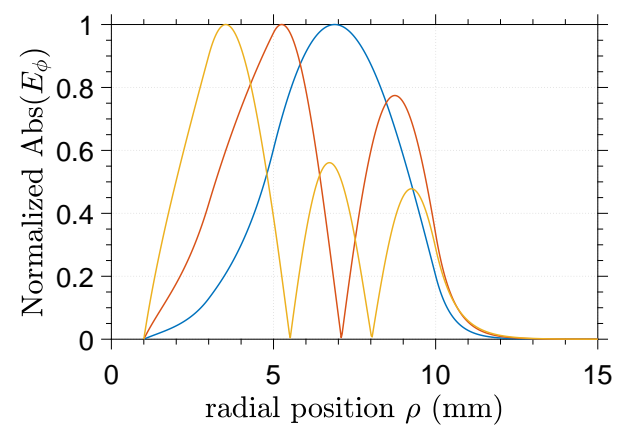

2.8(f): First three propagating modes of $E_{\phi}$ in $60 \mathrm{GHz}$

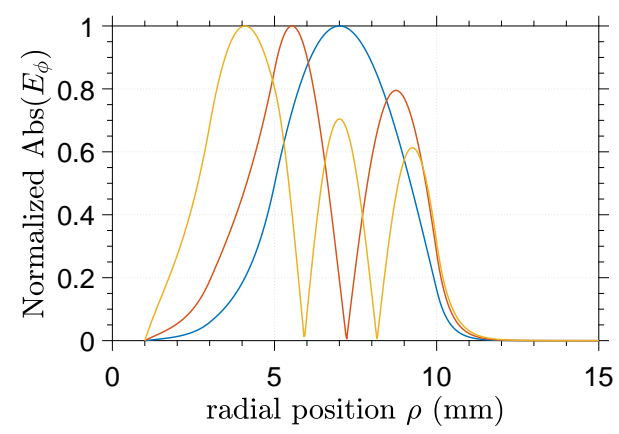

2.8(h): First three propagating modes of $E_{\phi}$ in $80 \mathrm{GHz}$

Figure 2.8: Normalized $E_{z}$ and $E_{\phi}$ fields of the first propagating modes in waveguide 1 at different operating frequencies. 


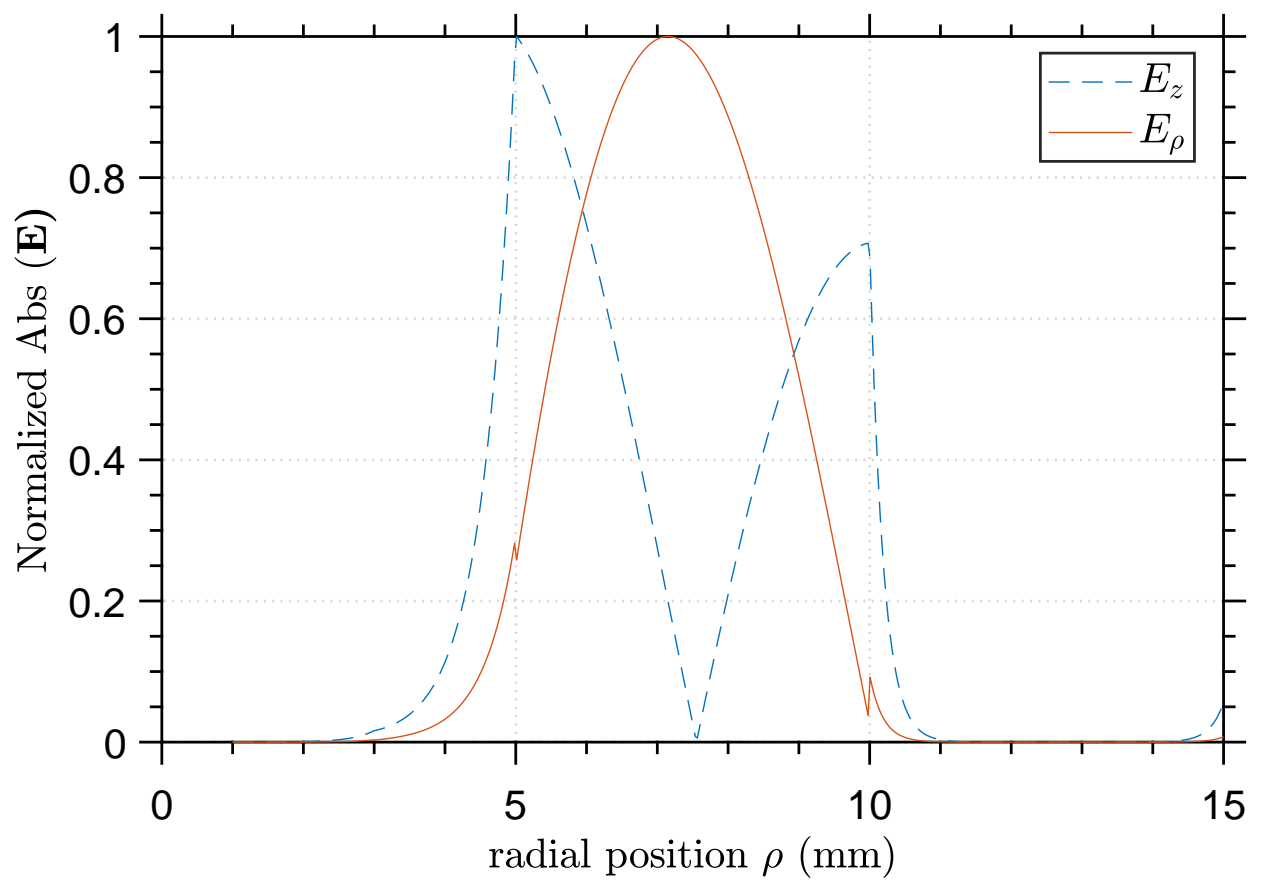

Figure 2.9: Normalized $E_{z}$ and $E_{\rho}$ fields of the first propagating mode in waveguide 1 in $160 \mathrm{GHz}$. Discontinuities of $E_{\rho}$ are smaller for higher frequencies. 


\section{Waveguide 2 - A three-layer anisotropic circular waveguide}

The second waveguide presented here is a three layers circular anisotropic waveguide defined by parameters in Table 2.5. The first nine wavenumbers for the frequencies 20, 40, 60 and $80 \mathrm{GHz}$ are in Fig 2.10. Fig 2.11 shows the normalized $E_{z}$ and $E_{\rho}$ field components of the fundamental mode. As occurred in the coaxial guide, the $\rho$ component of the field is discontinuous at the interfaces while the $E_{z}$ components are continuous.

Fig. 2.12 presents the fields for the first three TE $\left(E_{z}\right)$ and TM $\left(E_{\phi}\right)$ modes for the waveguide 2. Again, we can verify that the boundary conditions enforcement are correct.

The observation made before about the diminishing step discontinuity

\begin{tabular}{ccccc}
\hline Parameters & \multicolumn{4}{c}{ Layers } \\
\hline$\epsilon_{r s}$ & 1 & 2 & 3 & - \\
$\epsilon_{r z}$ & 1.5 & 2.55 & 4 & - \\
$\rho(\mathrm{mm})$ & 0 & 2 & 3.5 & 5 \\
\hline \multicolumn{5}{c}{$\sigma=10^{-6}(\mathrm{~S} / \mathrm{m})$} \\
\hline
\end{tabular}

Table 2.5: Dimensions and parameters of waveguide 2. Other constitutive parameters are equal to those of vacuum.

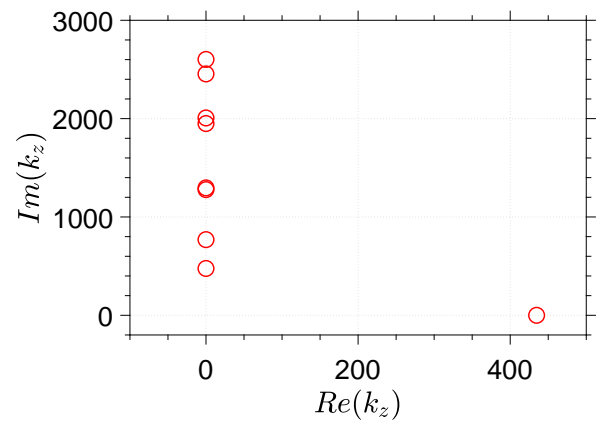

2.10(a): $20 \mathrm{GHz}$

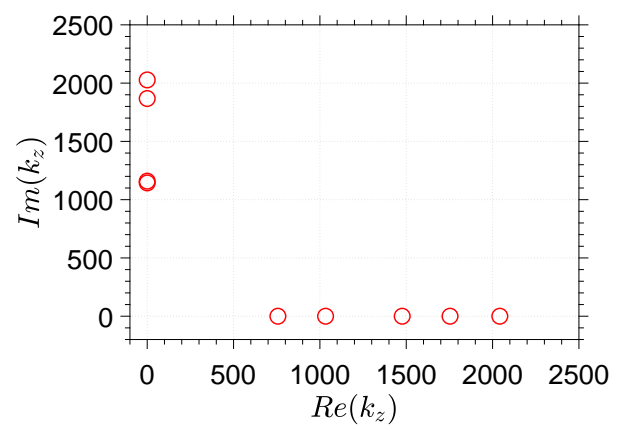

2.10(c): $60 \mathrm{GHz}$

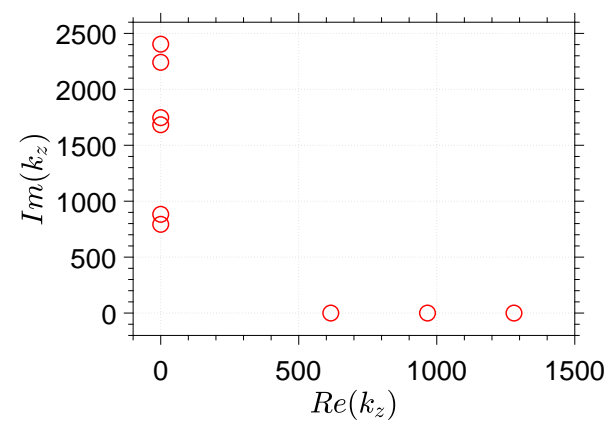

2.10(b): $40 \mathrm{GHz}$

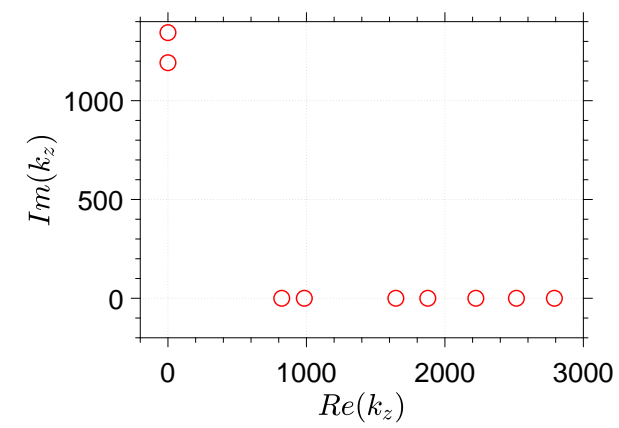

2.10(d): $80 \mathrm{GHz}$

Figure 2.10: First nine wavenumbers $k_{z}$ for waveguide 2 at different operating frequencies. 


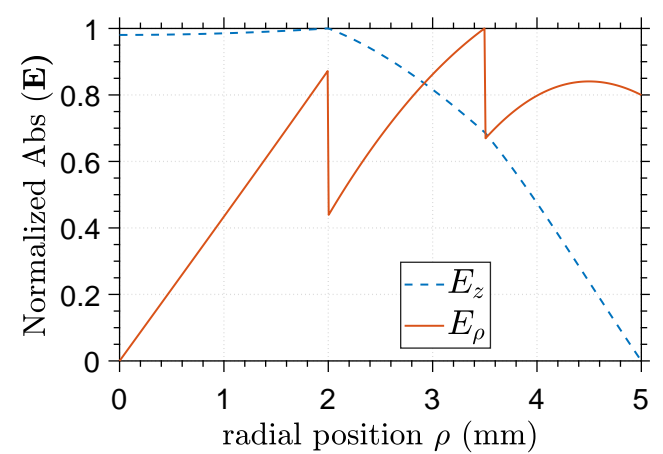

2.11(a): $20 \mathrm{GHz}$

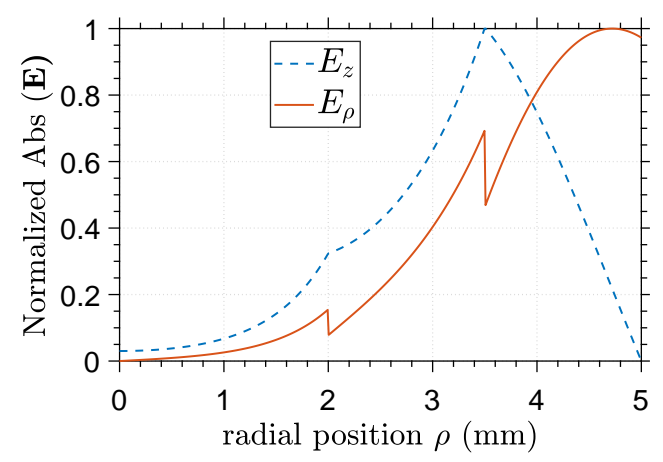

2.11(c): $60 \mathrm{GHz}$

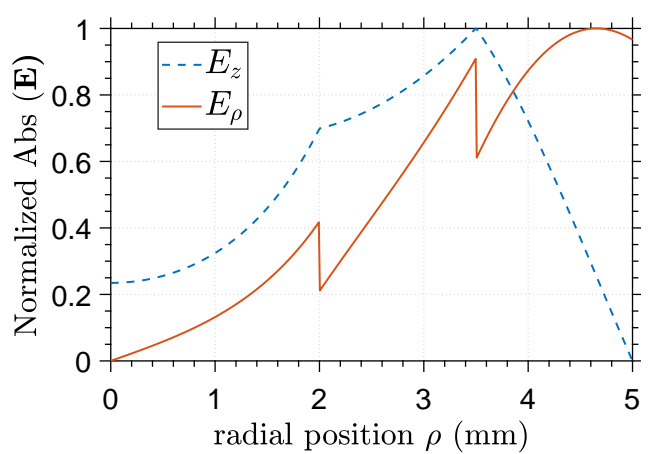

2.11(b): $40 \mathrm{GHz}$

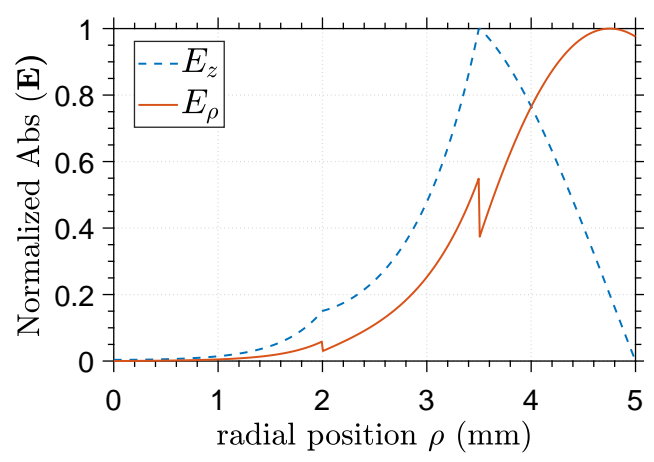

2.11(d): $80 \mathrm{GHz}$

Figure 2.11: Normalized $E_{z}$ and $E_{\rho}$ fields of the first propagating mode in waveguide 2 at different operating frequencies.

on the $\rho$ component of the electric field, apply, again, for this scenario, as seen in the field results at $160 \mathrm{GHz}$ shown in Fig 2.13. 


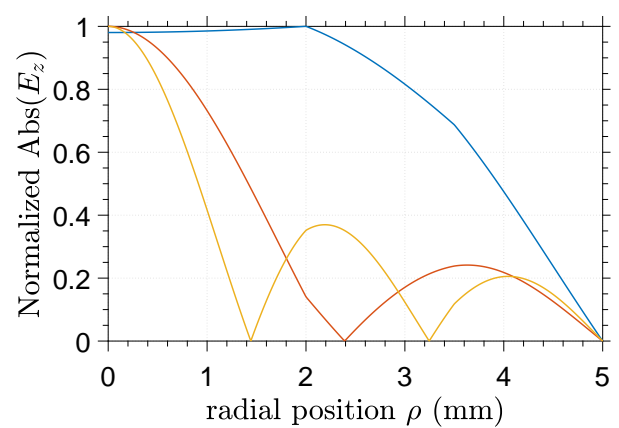

2.12(a): First three propagating modes of $E_{z}$ at $20 \mathrm{GHz}$

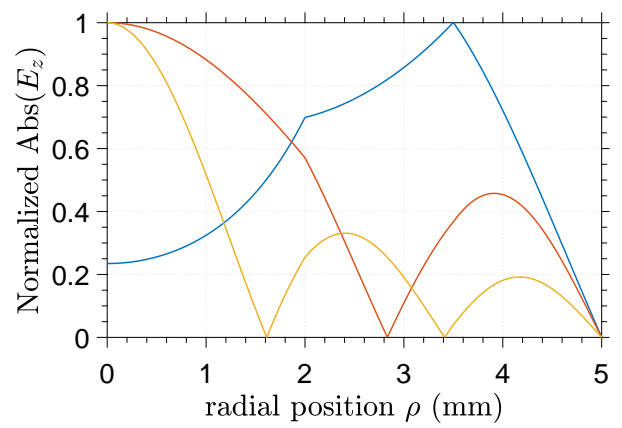

2.12(c): First three propagating modes of $E_{z}$ at $40 \mathrm{GHz}$

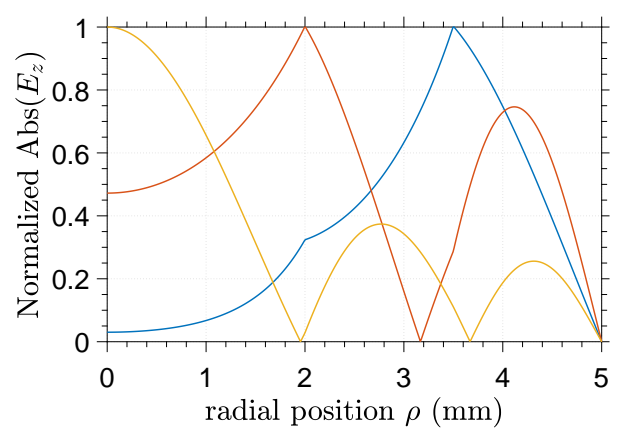

2.12(e): First three propagating modes of $E_{z}$ at $60 \mathrm{GHz}$

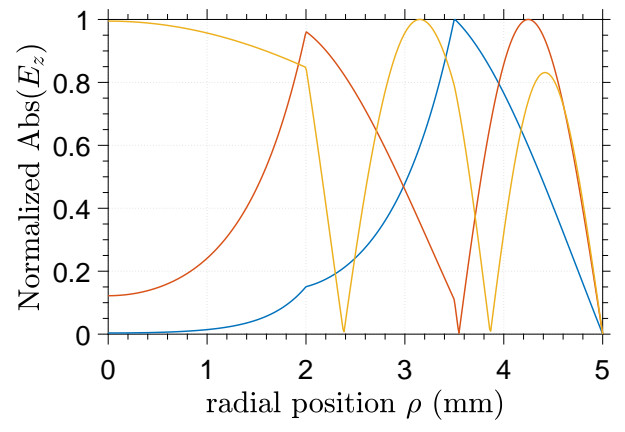

2.12(g): First three propagating modes of $E_{z}$ at $80 \mathrm{GHz}$

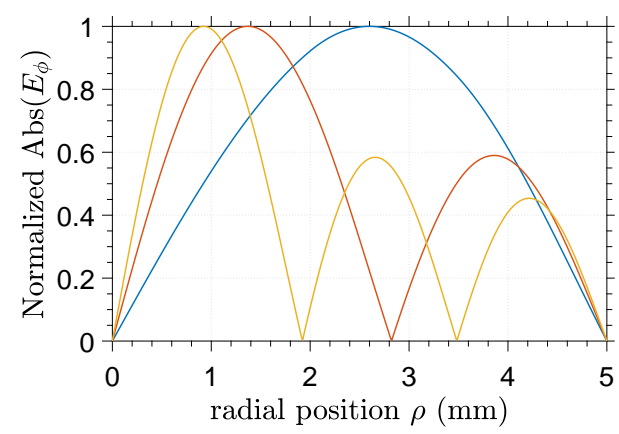

2.12(b): First three propagating modes of $E_{\phi}$ at $20 \mathrm{GHz}$

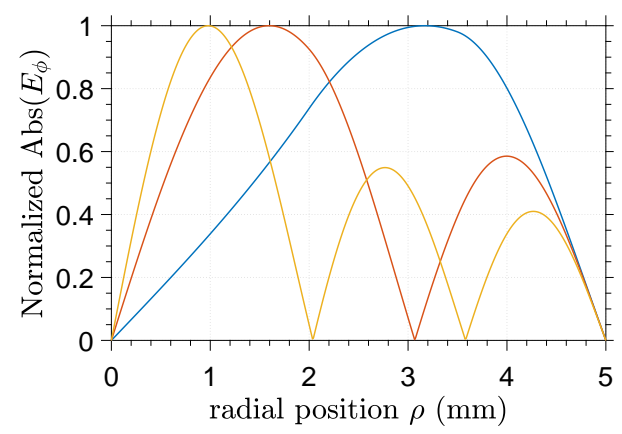

2.12(d): First three propagating modes of $E_{\phi}$ at $40 \mathrm{GHz}$

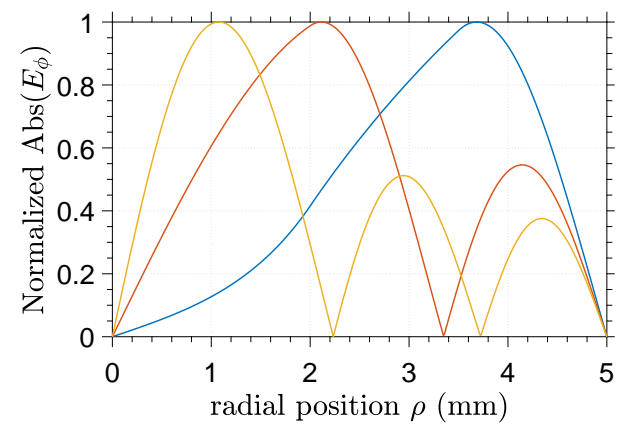

2.12(f): First three propagating modes of $E_{\phi}$ at $60 \mathrm{GHz}$

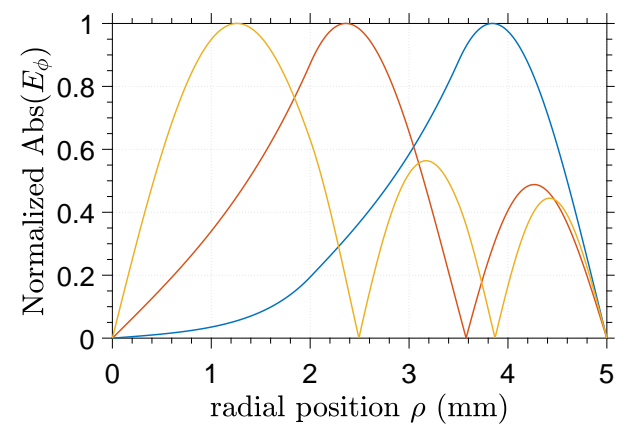

2.12(h): First three propagating modes of $E_{\phi}$ at $80 \mathrm{GHz}$

Figure 2.12: Normalized $E_{z}$ and $E_{\phi}$ fields of the first propagating modes in waveguide 2 at different operating frequencies. 


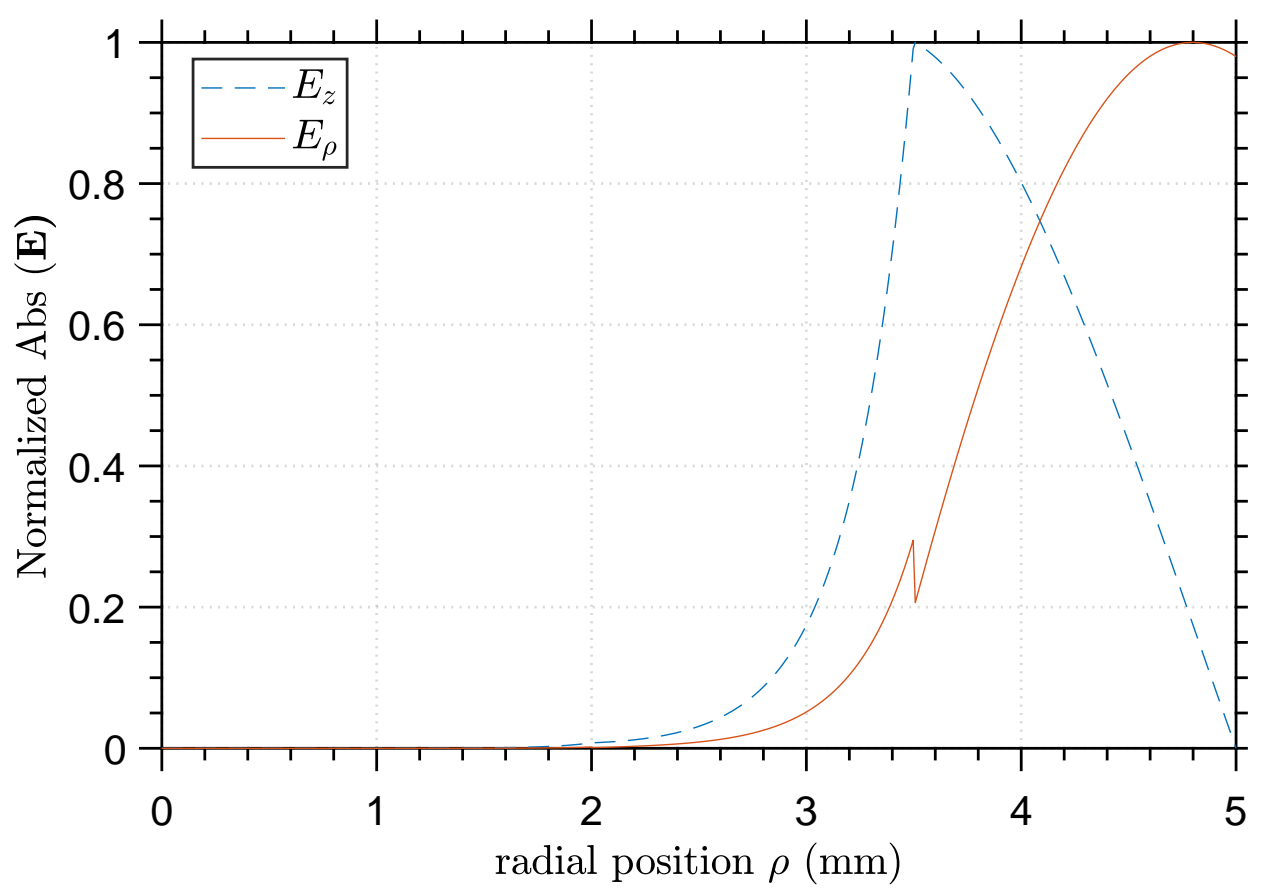

Figure 2.13: Normalized $E_{z}$ and $E_{\rho}$ fields of the first propagating mode in waveguide 2 at $160 \mathrm{GHz}$. Discontinuities of $E_{\rho}$ are smaller for higher frequencies.

\section{Field behavior inside dielectric layers}

The three-layer circular waveguide is now evaluated in three different scenarios, where only one layer is filled with a dielectric material (with $\epsilon_{r}=3$ ) and the other ones are vacuum. Fig. 2.14 shows the normalized $E_{\phi}$ field component of the main propagating mode at $100 \mathrm{GHz}$. We can observe that this field clearly concentrates on the layer filled by the dielectric material.

Also, to explore large dielectric contrast layers, consider the last waveguide has an isotropic dielectric material with $\epsilon_{r}=\{2,3,6,12\}$ filling only the outermost radial layer; the remaining are filled with vacuum. From Fig 2.15, we can observe that as the electric permittivity increases, the field become more concentrated in the dielectric layer, which is in accordance with results from [69]. 


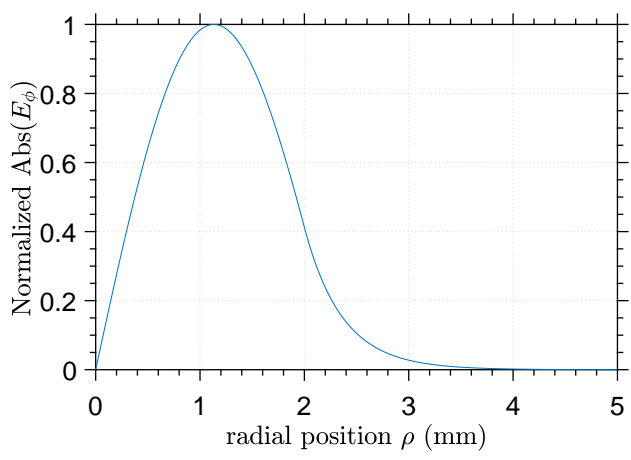

2.14(a): Dielectric in the first layer.

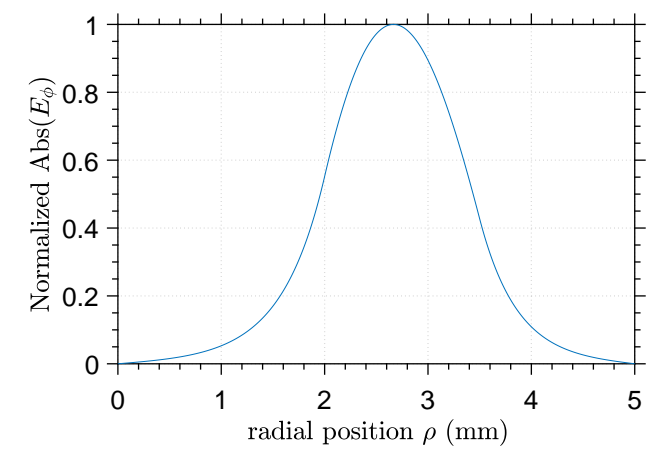

2.14(b): Dielectric in the second layer.

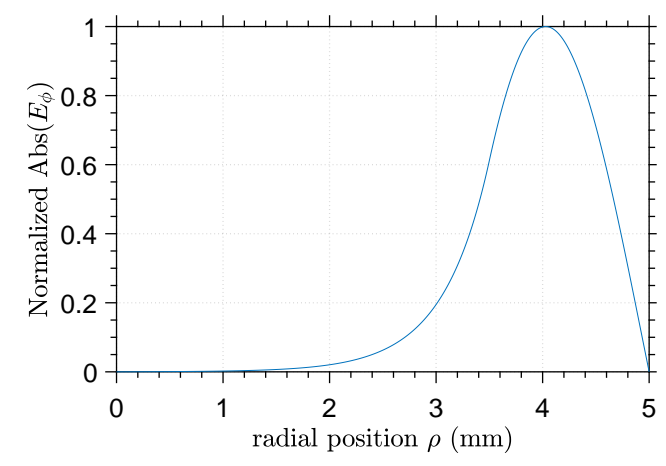

2.14(c): Dielectric in third layer.

Figure 2.14: Normalized $E_{\phi}$ fields of the main propagating mode in a threelayer circular waveguide at $100 \mathrm{GHz}$. A dielectric media (with $\epsilon_{r}=3$ ) is placed in the innermost, middle, and outermost radial layers. 


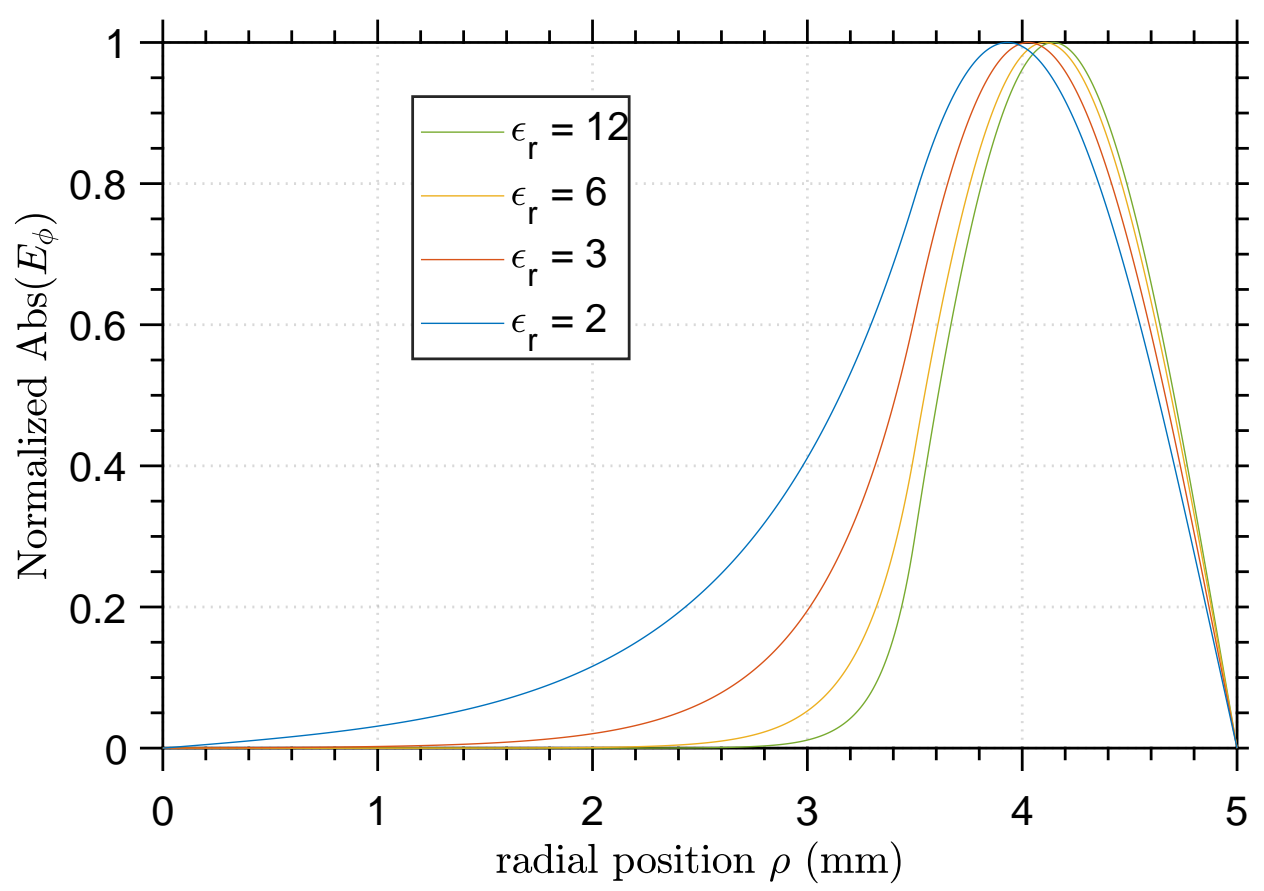

Figure 2.15: Normalized $E_{\phi}$ field component of the main propagating mode in a (virtual) three-layer circular waveguide at $100 \mathrm{GHz}$. The outermost radial layer is filled with a material characterized by the electric permittivity $\epsilon_{r}$. 


\section{Waveguide 3 - A five-layer coaxial waveguide}

Consider now the case Waveguide 3 . This is a five-layer coaxial waveguide with parameters shown in Table 2.6. In this case, we have an isotropic and lossy media, since $\sigma$ is non-null. As a consequence, the modal propagation constants $k_{z}$ will present both real and imaginary part. The corresponding eigenvalues $k_{z}$ were plotted in Fig 2.16. Fields $E_{z}$ and $E_{\rho}$ are in Fig 2.17, and the three first TE and TM modes of $z$ and $\phi$ components of the electric field are presented in Fig 2.18.

Observing $E_{\rho}$ and $E_{z}$ in Fig 2.17, we can see that the fields have a peculiar behavior: In each layer they have different oscillatory patterns. Also, as expected, all results are satisfying the interface boundary conditions.

\begin{tabular}{ccccccc}
\hline Parameters & \multicolumn{7}{c}{ Layers } \\
\hline$\epsilon_{r}$ & 3 & 5 & 2 & 3 & 2 & - \\
$\rho(\mathrm{mm})$ & 1 & 5 & 10 & 15 & 20 & 30 \\
$\sigma(\mathrm{S} / \mathrm{m})$ & 1 & 1.5 & 0.5 & 1 & 0.5 & - \\
\hline
\end{tabular}

Table 2.6: Dimensions and parameters of waveguide 3.

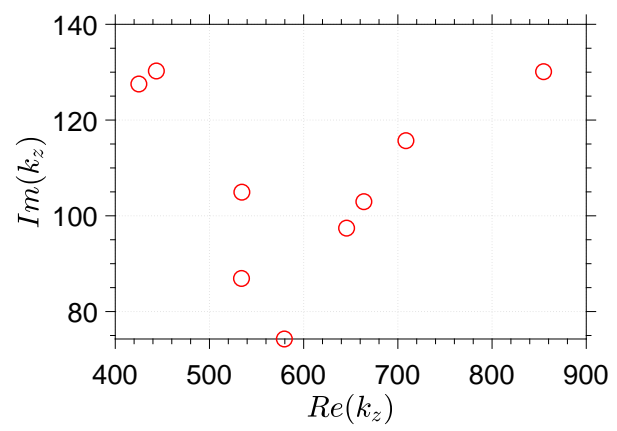

2.16(a): $20 \mathrm{GHz}$

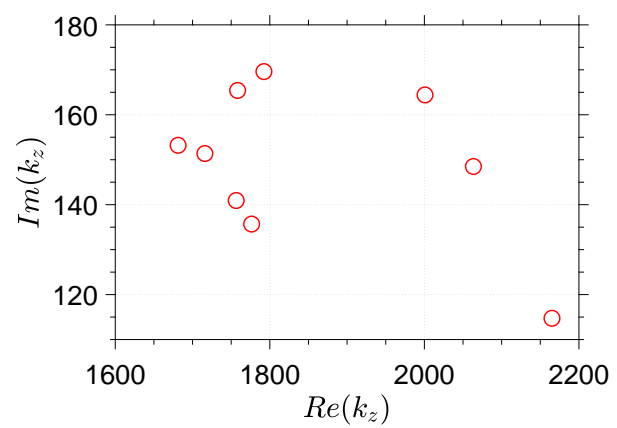

$2.16(\mathrm{c}): 60 \mathrm{GHz}$

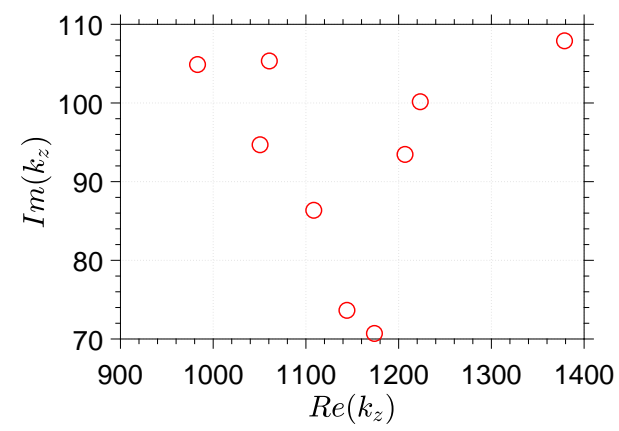

2.16(b): $40 \mathrm{GHz}$

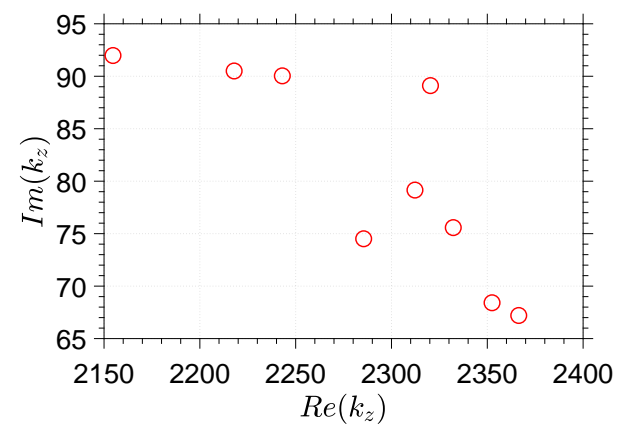

2.16(d): $80 \mathrm{GHz}$

Figure 2.16: First nine wavenumbers $k_{z}$ for waveguide 3 at different operating frequencies. 


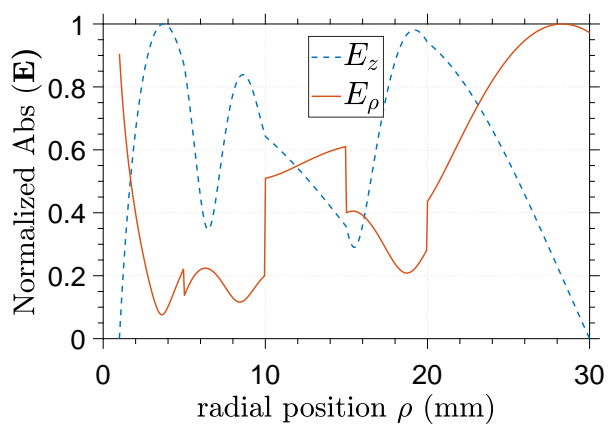

2.17(a): $20 \mathrm{GHz}$

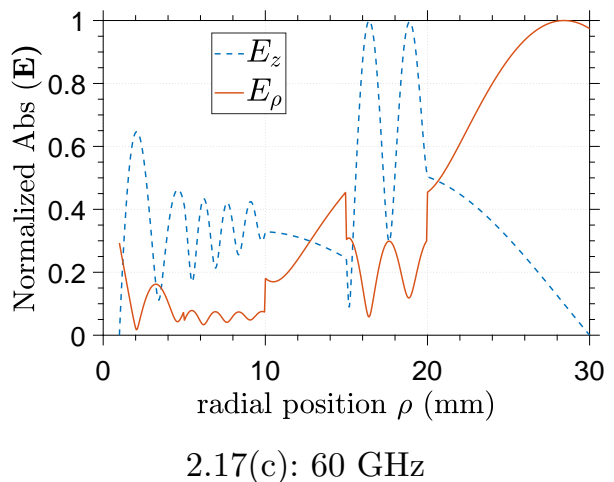

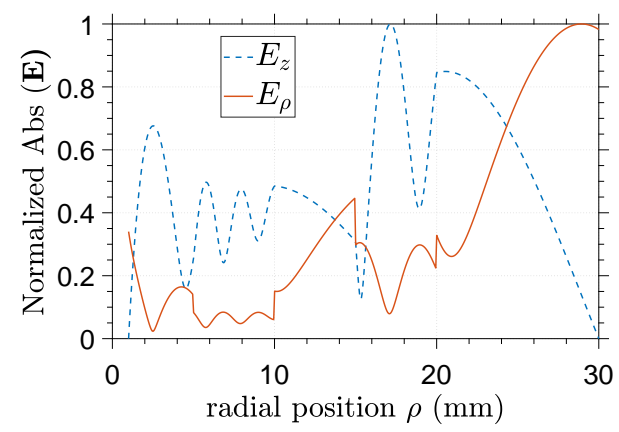

$2.17(\mathrm{~b}): 40 \mathrm{GHz}$

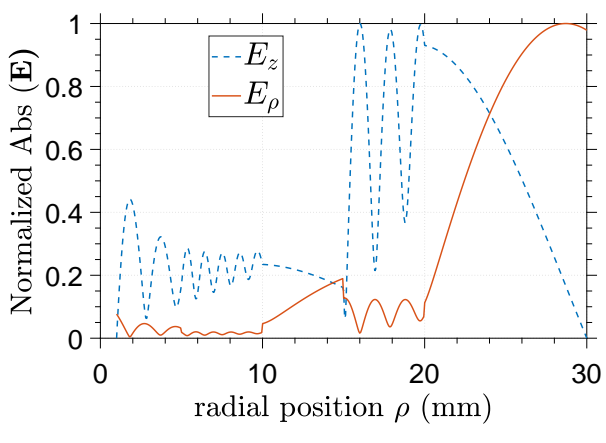

$2.17(\mathrm{~d}): 80 \mathrm{GHz}$

Figure 2.17: Normalized $E_{z}$ and $E_{\rho}$ fields of the first propagating mode in waveguide 3 at different operating frequencies. 


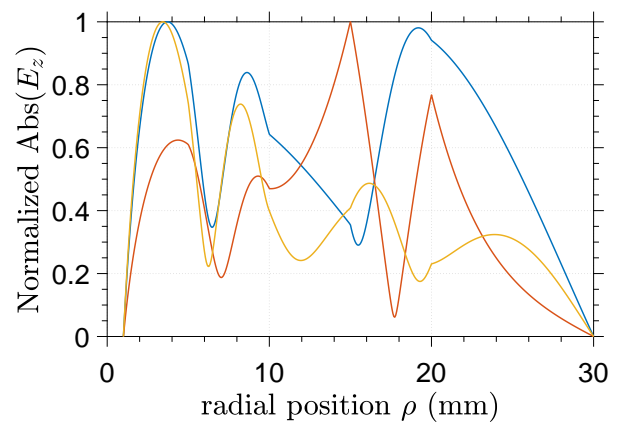

2.18(a): First three propagating modes of $E_{z}$ at $20 \mathrm{GHz}$

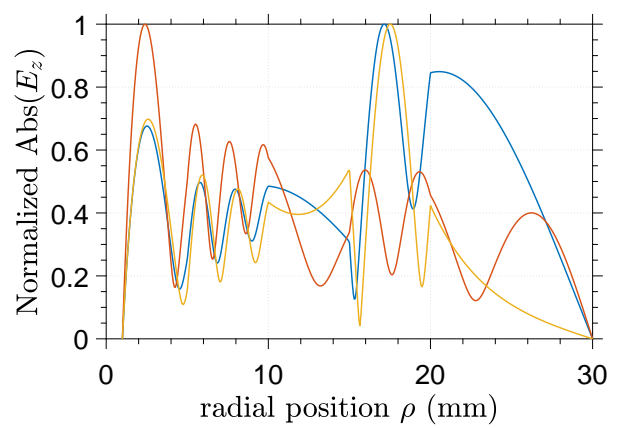

2.18(c): First three propagating modes of $E_{z}$ at $40 \mathrm{GHz}$

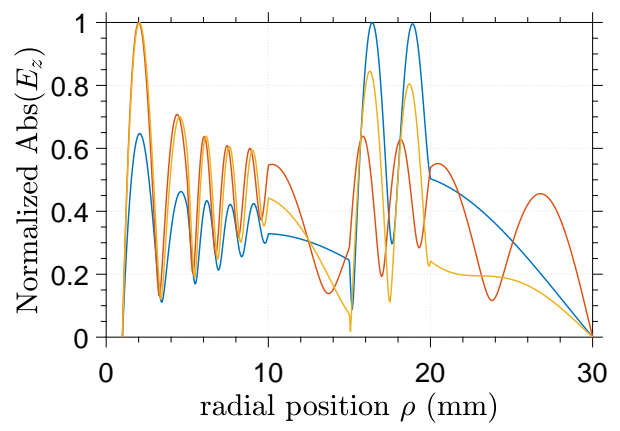

2.18(e): First three propagating modes of $E_{z}$ at $60 \mathrm{GHz}$

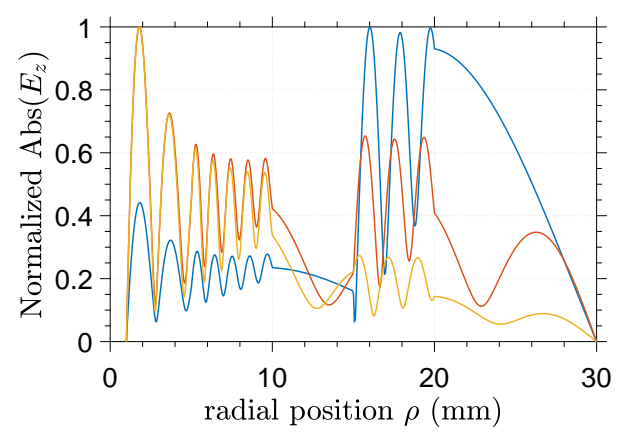

2.18(g): First three propagating modes of $E_{z}$ at $80 \mathrm{GHz}$

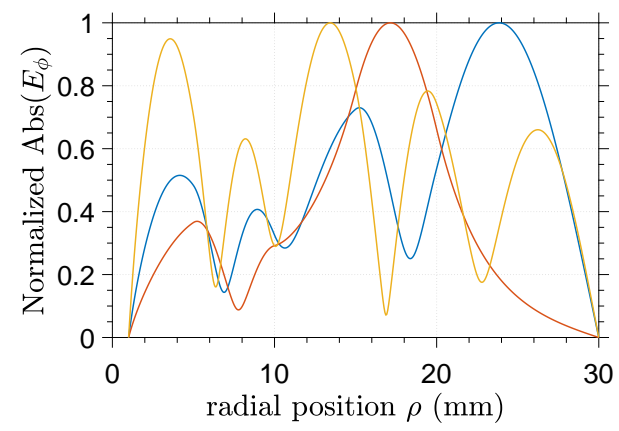

2.18(b): First three propagating modes of $E_{\phi}$ at $20 \mathrm{GHz}$

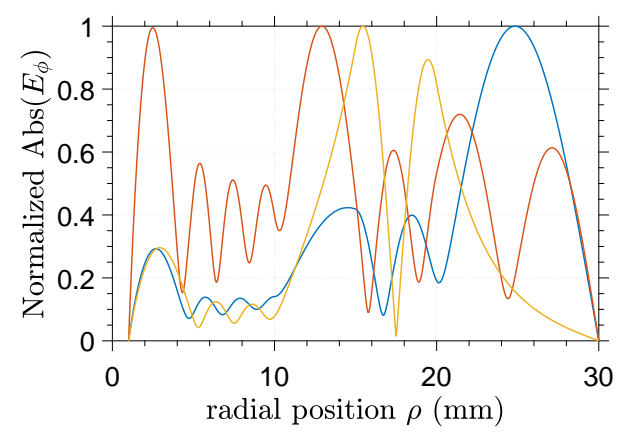

2.18(d): First three propagating modes of $E_{\phi}$ at $40 \mathrm{GHz}$

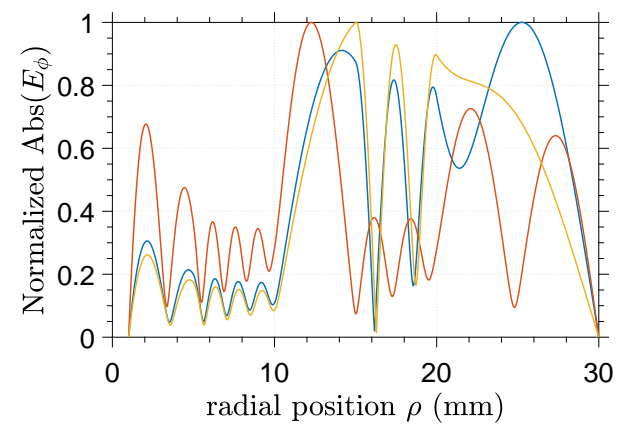

2.18(f): First three propagating modes of $E_{\phi}$ at $60 \mathrm{GHz}$

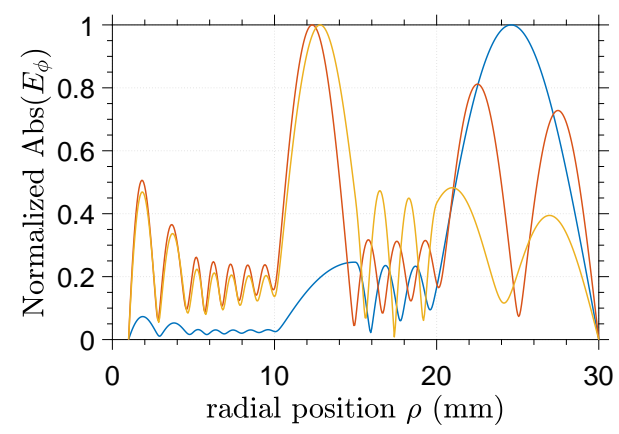

2.18(h): First three propagating modes of $E_{\phi}$ at $80 \mathrm{GHz}$

Figure 2.18: Normalized $E_{z}$ and $E_{\phi}$ fields of the first propagating modes in waveguide 3 at different operating frequencies. 


\section{Waveguide 4 - A six-layer circular waveguide}

Consider now the scenario of waveguide 4 , formed by a six-layer circular waveguide with variations on the electrical conductivity along de radial layers, according to the parameters shown in Table 2.7. Fig 2.19 shows the eigenvalues we have found. The first mode of normalized $E_{z}$ and $E_{\rho}$ fields are in Fig 2.20 and results are in accordance with the previous cases.

Fig. 2.21 presents the $z$ and $\phi$ electric field components for the first TE and TM modes for the same frequencies as in the other examples. As expected, all the boundary conditions are continuous across the radial interfaces.

\begin{tabular}{cccccccc}
\hline Parameters & \multicolumn{7}{c}{ Layers } \\
\hline$\epsilon_{r}$ & 2.55 & 4 & 2.55 & 4 & 2.55 & 4 & - \\
$\rho(\mathrm{mm})$ & 0 & 5 & 10 & 15 & 20 & 25 & 30 \\
\hline \multicolumn{7}{c}{$\sigma=1(\mathrm{~S} / \mathrm{m})$} \\
\hline
\end{tabular}

Table 2.7: Dimensions and parameters of waveguide 4 .

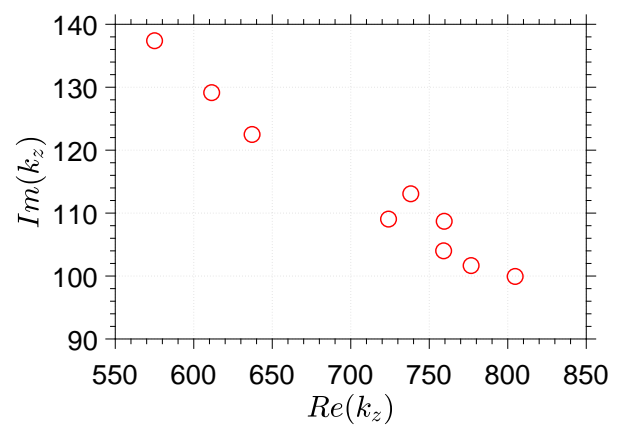

2.19(a): $20 \mathrm{GHz}$

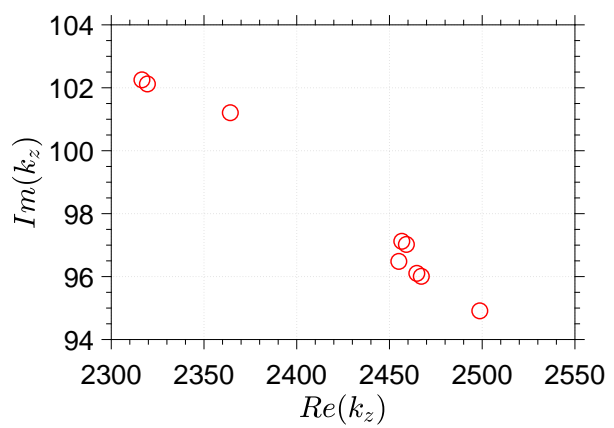

2.19(c): $60 \mathrm{GHz}$

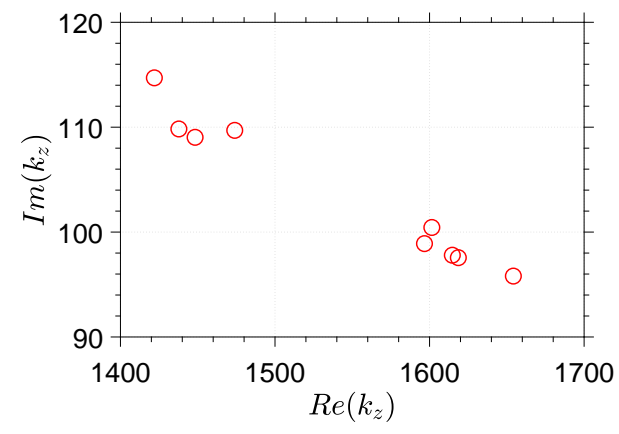

2.19(b): $40 \mathrm{GHz}$

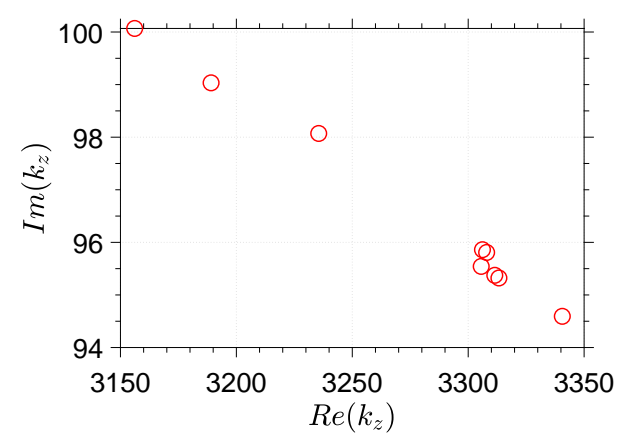

2.19(d): $80 \mathrm{GHz}$

Figure 2.19: First nine wavenumbers $k_{z}$ for waveguide 4 at different operating frequencies. 


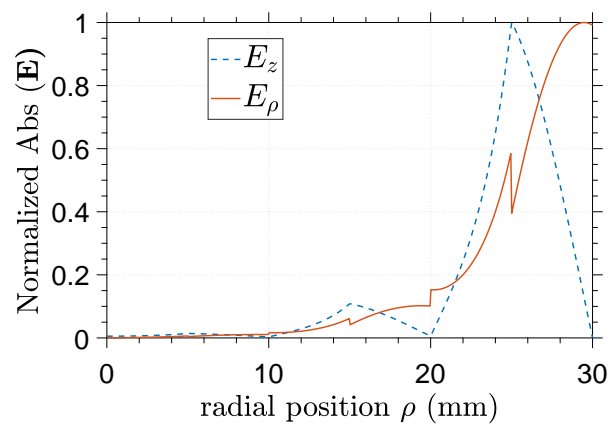

2.20(a): $20 \mathrm{GHz}$

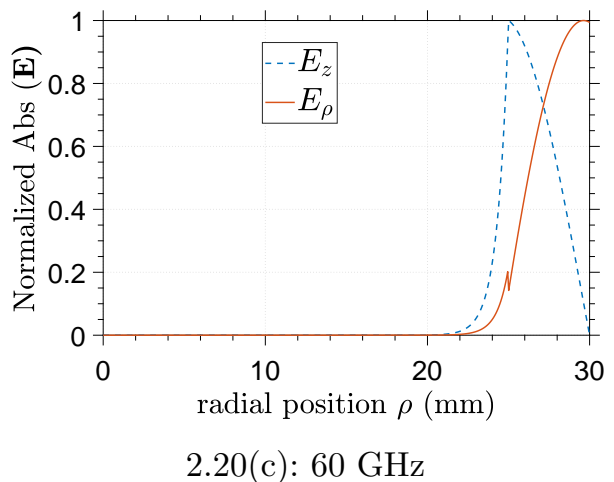

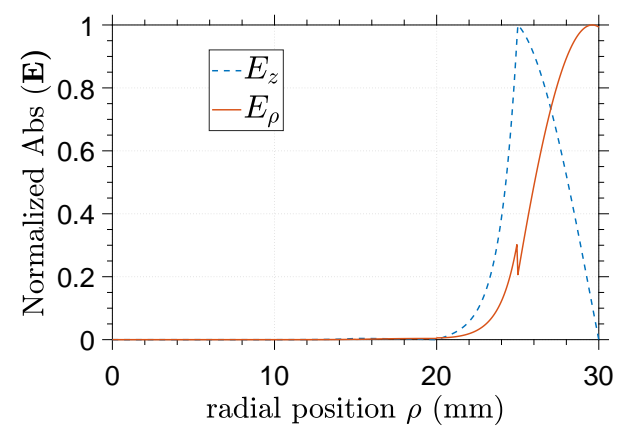

2.20(b): $40 \mathrm{GHz}$

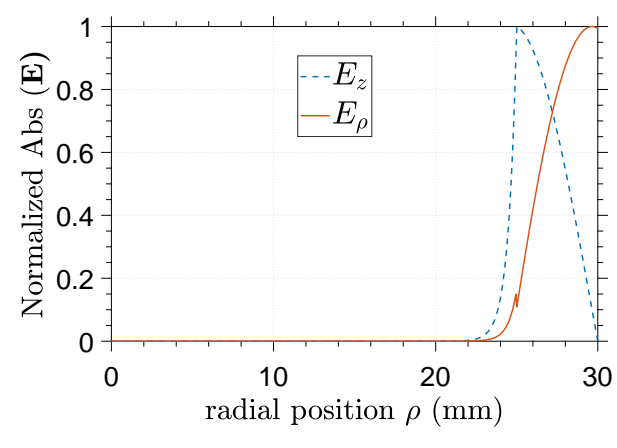

$2.20(\mathrm{~d}): 80 \mathrm{GHz}$

Figure 2.20: Normalized $E_{z}$ and $E_{\rho}$ fields of the first propagating mode in waveguide 4 at different operating frequencies. 


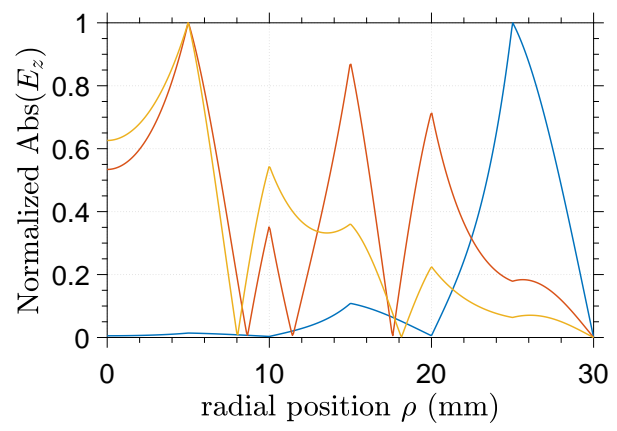

2.21(a): First three propagating modes of $E_{z}$ at $20 \mathrm{GHz}$

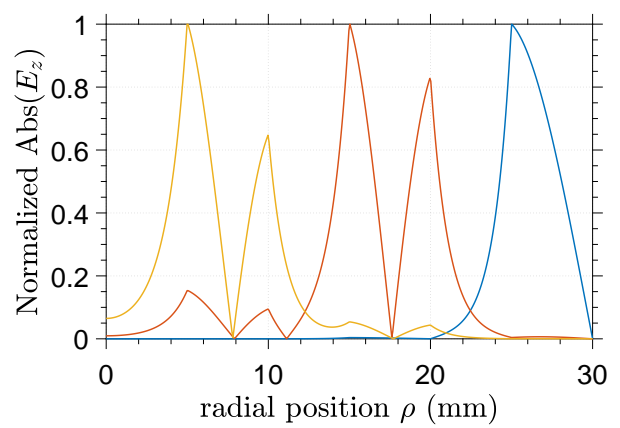

2.21(c): First three propagating modes of $E_{z}$ at $40 \mathrm{GHz}$

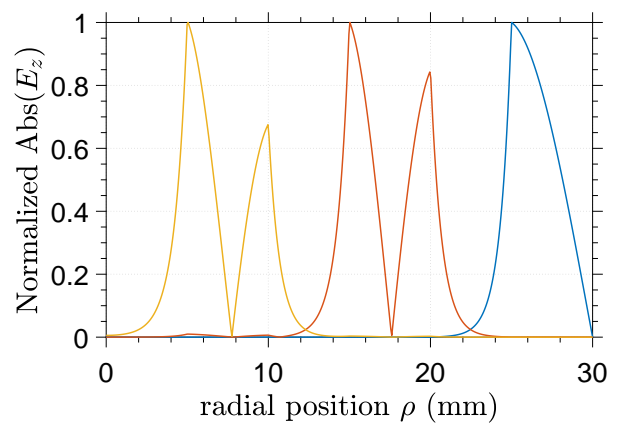

2.21(e): First three propagating modes of $E_{z}$ at $60 \mathrm{GHz}$

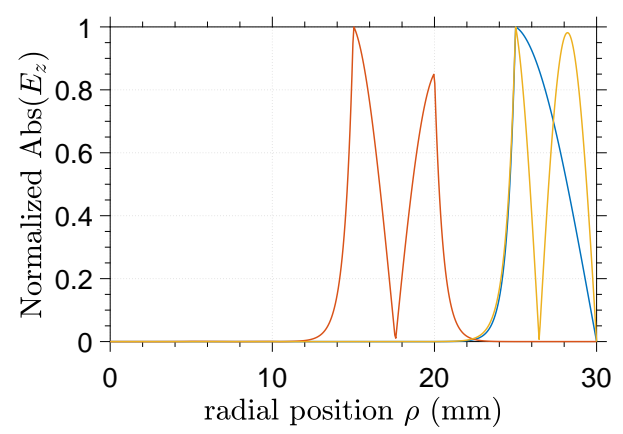

2.21(g): First three propagating modes of $E_{z}$ at $80 \mathrm{GHz}$

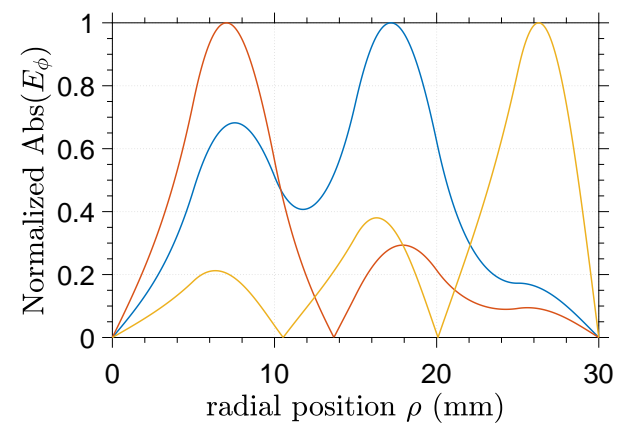

2.21(b): First three propagating modes of $E_{\phi}$ at $20 \mathrm{GHz}$

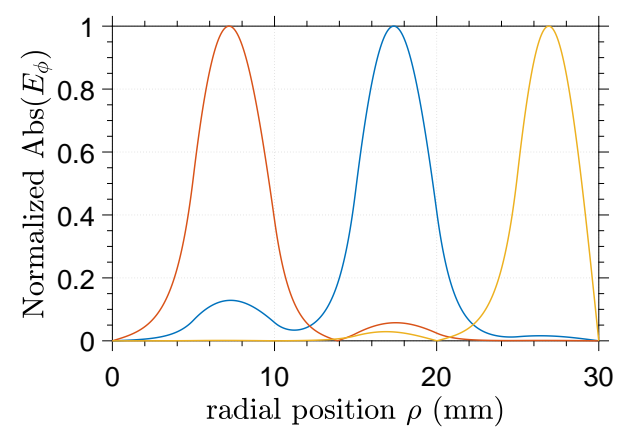

2.21(d): First three propagating modes of $E_{\phi}$ at $40 \mathrm{GHz}$

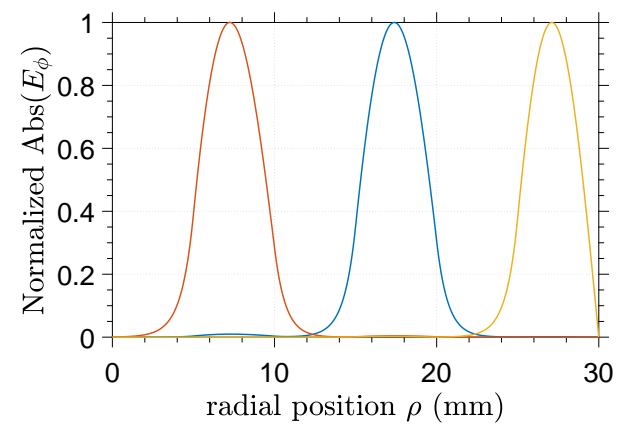

2.21(f): First three propagating modes of $E_{\phi}$ at $60 \mathrm{GHz}$

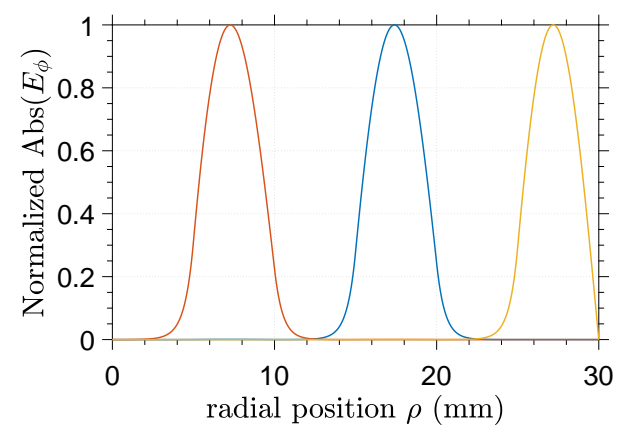

2.21(h): First three propagating modes of $E_{\phi}$ at $80 \mathrm{GHz}$

Figure 2.21: Normalized $E_{z}$ and $E_{\phi}$ fields of the first propagating modes in waveguide 4 at different operating frequencies. 


\section{Modal field concentration on the waveguide layers}

It is possible to observe from examples of waveguides 1, 2 and 4, that, as the frequency increases, the fields associated to the fundamental modes becomes concentrated in the layer with the highest dielectric permittivity. This is on the field component $E_{\phi}$. Also, the second mode concentrates on the layer with the second highest permittivity, and third mode in the layer with third highest one. As critical case, for waveguide 4, we can notice that the fields at $80 \mathrm{GHz}$, for the first three propagation modes, become almost orthogonal to each other. In other words, there is a very small overlapping between the fields along rho.

For a waveguide with the same value of $\sigma$ in all the layers, we can predict the following: at lower frequencies, the power associated to a modal field will be diffuse along the radial domain, but at higher frequencies, the power will concentrate in the layer with the higher dense media. In order to verify this, an additional example is evaluated. Waveguide 4 is now modified according to the parameters listed in Table 2.8. Notice here we are considering some anisotropic layers.

Fig. 2.22 presents the $E_{z}$ fields for $12 \mathrm{TM}$ modes, and the $E_{\phi}$ fields for 10 TE modes at different operating frequencies. We can observe the behavior we have predicted previously for the high-order modal fields. By examining $E_{\phi}$, we notice that a large number of TE modes concentrates the power into two more dense layers at $80 \mathrm{GHz}$. Such are the anisotropic layers. By examining $E_{z}$ field component, we can observe that TM modes presents an opposite behavior: the layer with higher anisotropic permittivity (with $\epsilon_{r s}=4$ and $\epsilon_{r z}=6$ ) is the one where fields are less concentrated. It is important to mention such results are for the normalized fields with respect to its maximum values. The source boundary conditions were not yet imposed.

In the case of waveguide 3, the layers had different values of $\sigma$, and consequently, different attenuations are experienced along the radial domain. As the frequency increases, the fields intensity concentrates in the layers with the lowest electrical conductivity (where $\sigma=0.5$, i.e., $10 \mathrm{~mm} \leq \rho \leq 15 \mathrm{~mm}$

\begin{tabular}{cccccccc}
\hline Parameters & \multicolumn{8}{c}{ Layers } \\
\hline$\epsilon_{r s}$ & 2.55 & 4 & 2.55 & 4 & 2.55 & 4 & - \\
$\epsilon_{r z}$ & 2.55 & 6 & 2.55 & 5 & 2.55 & 4 & - \\
$\rho(\mathrm{mm})$ & 0 & 5 & 10 & 15 & 20 & 25 & 30 \\
\hline \multicolumn{7}{c}{$\sigma=1(\mathrm{~S} / \mathrm{m})$} \\
\hline
\end{tabular}

Table 2.8: Dimensions and parameters of waveguide 4 with anisotropic layers. 


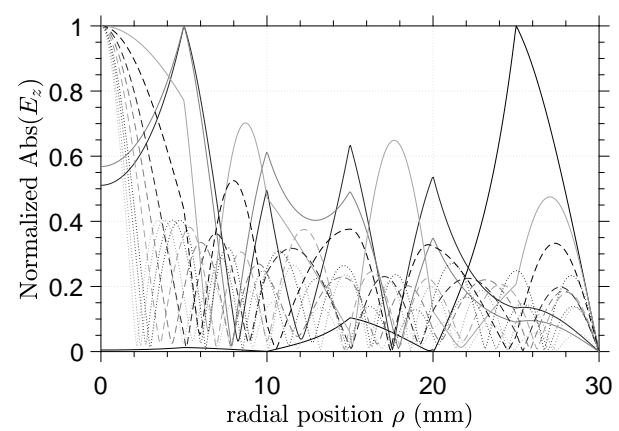

2.22(a): TM modes of $E_{z}$ at $20 \mathrm{GHz}$

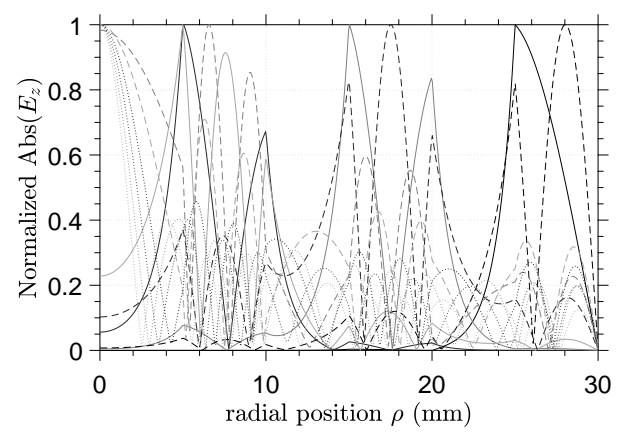

2.22(c): TM modes of $E_{z}$ at $40 \mathrm{GHz}$

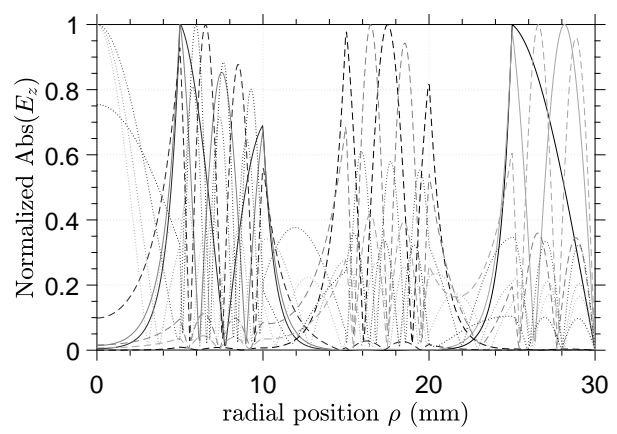

2.22(e): TM modes of $E_{z}$ at $60 \mathrm{GHz}$

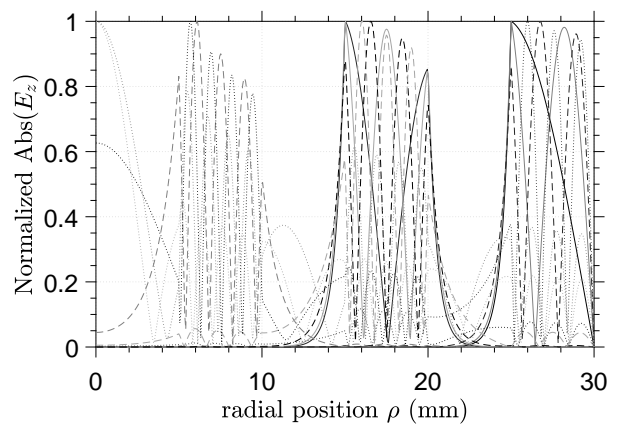

2.22(g): TM modes of $E_{z}$ at $80 \mathrm{GHz}$

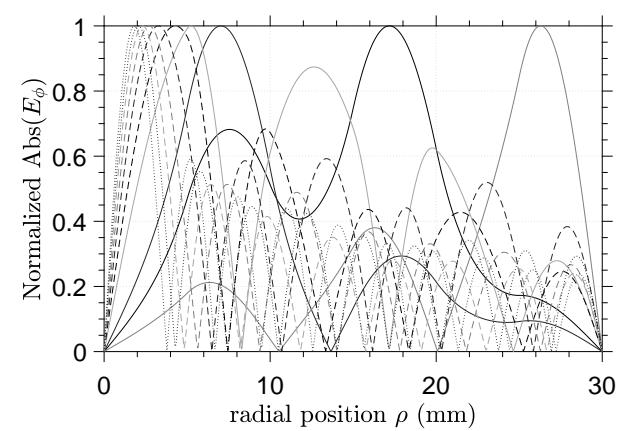

2.22(b): TE modes of $E_{\phi}$ at $20 \mathrm{GHz}$

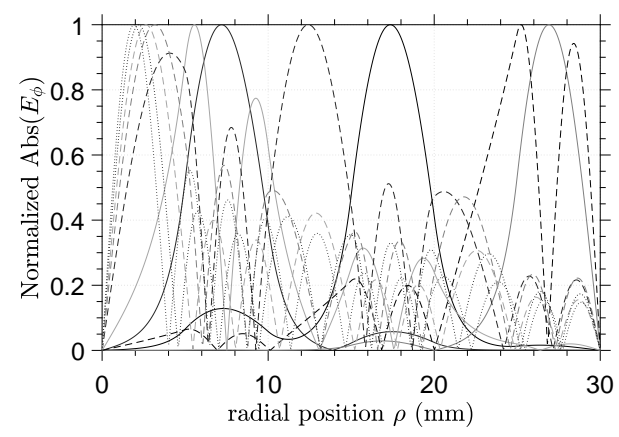

2.22(d): TE modes of $E_{\phi}$ at $40 \mathrm{GHz}$

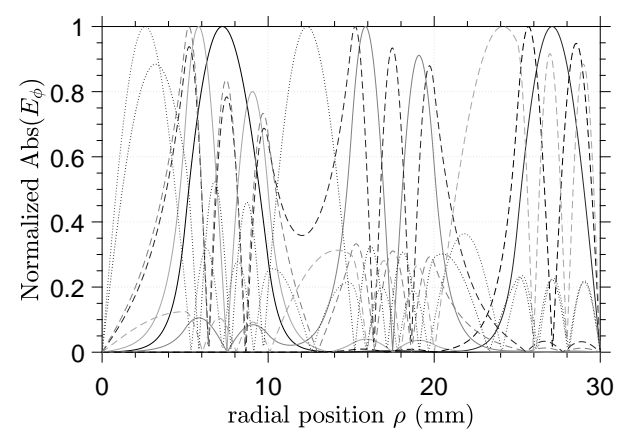

2.22(f): TE modes of $E_{\phi}$ at $60 \mathrm{GHz}$

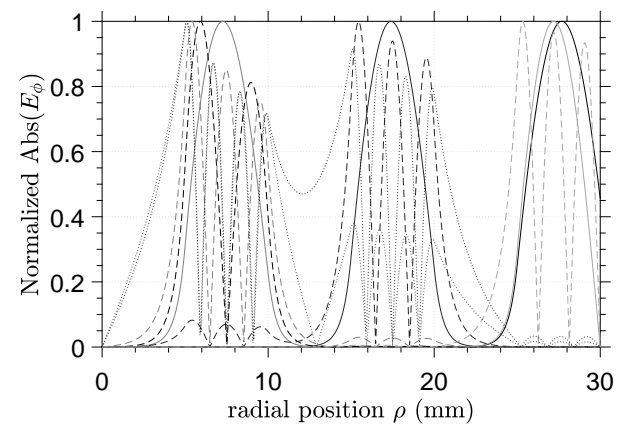

2.22(h): TE modes of $E_{\phi}$ at $80 \mathrm{GHz}$

Figure 2.22: Normalized $E_{z}$ and $E_{\phi}$ fields in an anisotropic waveguide 4 at different operating frequencies.

and $20 \mathrm{~mm} \leq \rho \leq 30 \mathrm{~mm}$ ). It is expected that in regions with lower losses the field pattern becomes concentrated.

Consider now a problem similar to waveguide 3, but with the updated 
parameters listed in Table 2.9 .

Figure 2.23 shows the computed fields for simulations 1 and 2 at $80 \mathrm{GHz}$. We are presenting $9 \mathrm{TE}$ and $9 \mathrm{TM}$ modes. In simulation 1, the layer with the highest value for $\epsilon_{r}$ had the lowest $\sigma$. In this case, both TE and TM fields are concentrated the in the layers with the highest $\epsilon_{r}$. This behavior was expected, since these layers present low-loss characteristic.

In simulation 2 (see Fig. 2.24), the layers with higher $\epsilon_{r}$ had also higher $\sigma$. We can observe that for $E_{z}$ fields, a slight concentration of modes in layers with high dielectric values occur, although the high losses. For the TE modes (characterized by the $E_{\phi}$ field), we cannot distinguish any clear pattern of concentration of the field. In a nutshell, for the TM modes, the radial concentration of the fields at high-frequencies are mainly due to the values of $\epsilon_{r}$ in a given layer, with a small correlation with $\sigma$. This could be explained because the conduction current is dominated by the displaced one at high-frequencies of operation.

\begin{tabular}{cccccccc}
\hline Simulation & Parameter & \multicolumn{7}{c}{ Layers } \\
\hline \multirow{3}{*}{1} & $\epsilon_{r}$ & 4 & 2 & 4 & 2 & 4 & - \\
& $\sigma(\mathrm{S} / \mathrm{m})$ & 0.1 & 1 & 0.1 & 1 & 0.1 & - \\
& $\rho(\mathrm{mm})$ & 1 & 5 & 10 & 15 & 20 & 30 \\
\hline \multirow{3}{*}{2} & $\epsilon_{r}$ & 4 & 2 & 4 & 2 & 4 & - \\
& $\sigma(\mathrm{S} / \mathrm{m})$ & 1 & 0.1 & 1 & 0.1 & 1 & - \\
& $\rho(\mathrm{mm})$ & 1 & 5 & 10 & 15 & 20 & 30 \\
\hline
\end{tabular}

Table 2.9: Dimensions and parameters of waveguides used in simulation 1 and 2 .

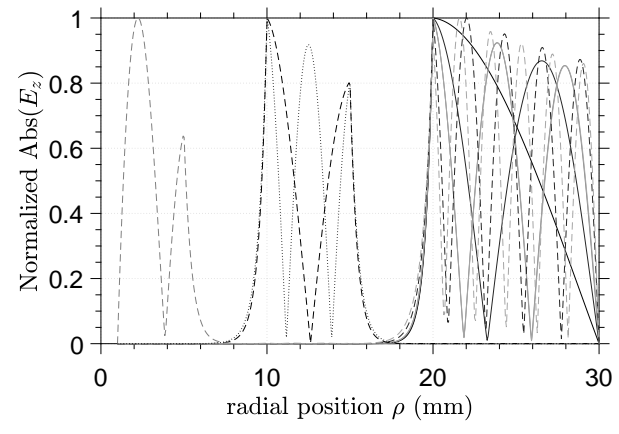

2.23(a): TM modes of $E_{z}$

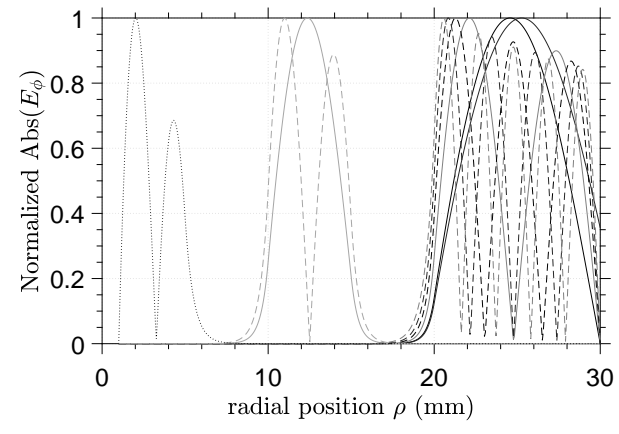

2.23(b): TE modes of $E_{\phi}$

Figure 2.23: Normalized $E_{z}$ and $E_{\phi}$ fields of simulation 1 at $80 \mathrm{GHz}$. 


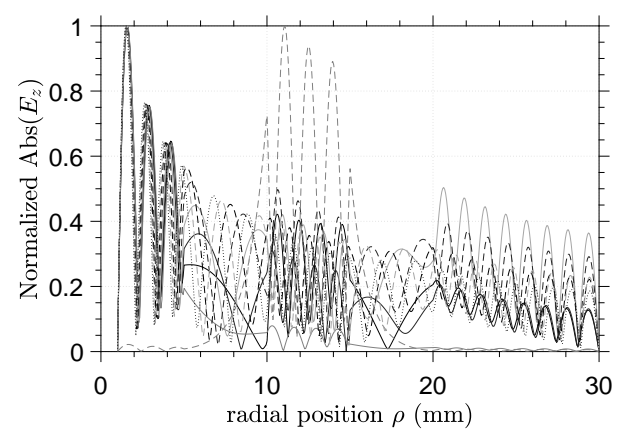

2.24(a): TM modes of $E_{z}$

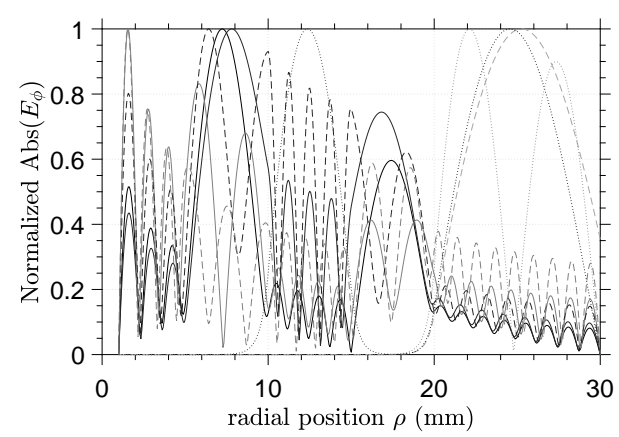

2.24(b): TE modes of $E_{\phi}$

Figure 2.24: Normalized $E_{z}$ and $E_{\phi}$ fields of simulation 2 at $80 \mathrm{GHz}$.

\section{Influence of anisotropy in field discontinuity}

In interfaces where exist a variation in the electric parameters of the media, we have discontinuous distributions of charges and currents [1]. The discontinuities in the $E_{\rho}$ field component observed in the previous examples are proportional to the permittivity contrast between adjacent layers.

To become possible to analyze the influence of the anisotropy in these discontinuities, a simulation was made considering Waveguide 5 (with parameters in Table 2.10), in $20 \mathrm{GHz}$. The values of $\epsilon_{r z}$ were varied according to Table 2.11 and the difference observed in the $\rho$ component is shown in Fig 2.25. Simulation 1 is equivalent to the isotropic case, while others have an increasing step of 0.5 .

After the first discontinuity at $2 \mathrm{~mm}$, the amplitude of the field becomes greater for higher values of $\epsilon_{r z}$. Also, we can observe that as the anisotropy becomes larger, the size of the discontinuity also grows. The step present at $\rho=3.5 \mathrm{~mm}$ has size equal to $0.0964 \mathrm{~V} / \mathrm{m}$ in simulation $1,0.1049 \mathrm{~V} / \mathrm{m}$ in simulation $2,0.1143 \mathrm{~V} / \mathrm{m}$ in simulation 3 and $0.1246 \mathrm{~V} / \mathrm{m}$ in simulation 4 . At the interface between layers 1 and 2 , the difference is not very clear, but from layer 2 to 3, this becomes more evident. From these simulations, it is possible to confirm that the size of the discontinuities in the $E_{\rho}$ field is related to the permittivity value and the degree of anisotropy of the material.

\begin{tabular}{ccccc}
\hline Parameters & \multicolumn{4}{c}{ Layers } \\
\hline$\epsilon_{r s}$ & 1 & 2 & 3 & - \\
$\epsilon_{r z}$ & 1.5 & 2.5 & 3.5 & - \\
$\rho(\mathrm{mm})$ & 1.5 & 2 & 3.5 & 5 \\
\hline \multicolumn{4}{c}{$\sigma=10^{-6}(\mathrm{~S} / \mathrm{m})$} \\
\hline
\end{tabular}

Table 2.10: Dimensions and parameters of waveguide 5 with anisotropic layers. 


\begin{tabular}{cccc}
\hline Simulation & \multicolumn{3}{c}{$\epsilon_{r z}$} \\
\hline 1 & 1 & 2 & 3 \\
2 & 1.5 & 2.5 & 3.5 \\
3 & 2 & 3 & 4 \\
4 & 2.5 & 3.5 & 4.5 \\
\hline
\end{tabular}

Table 2.11: Values of $\epsilon_{r z}$ for Waveguide 5 .

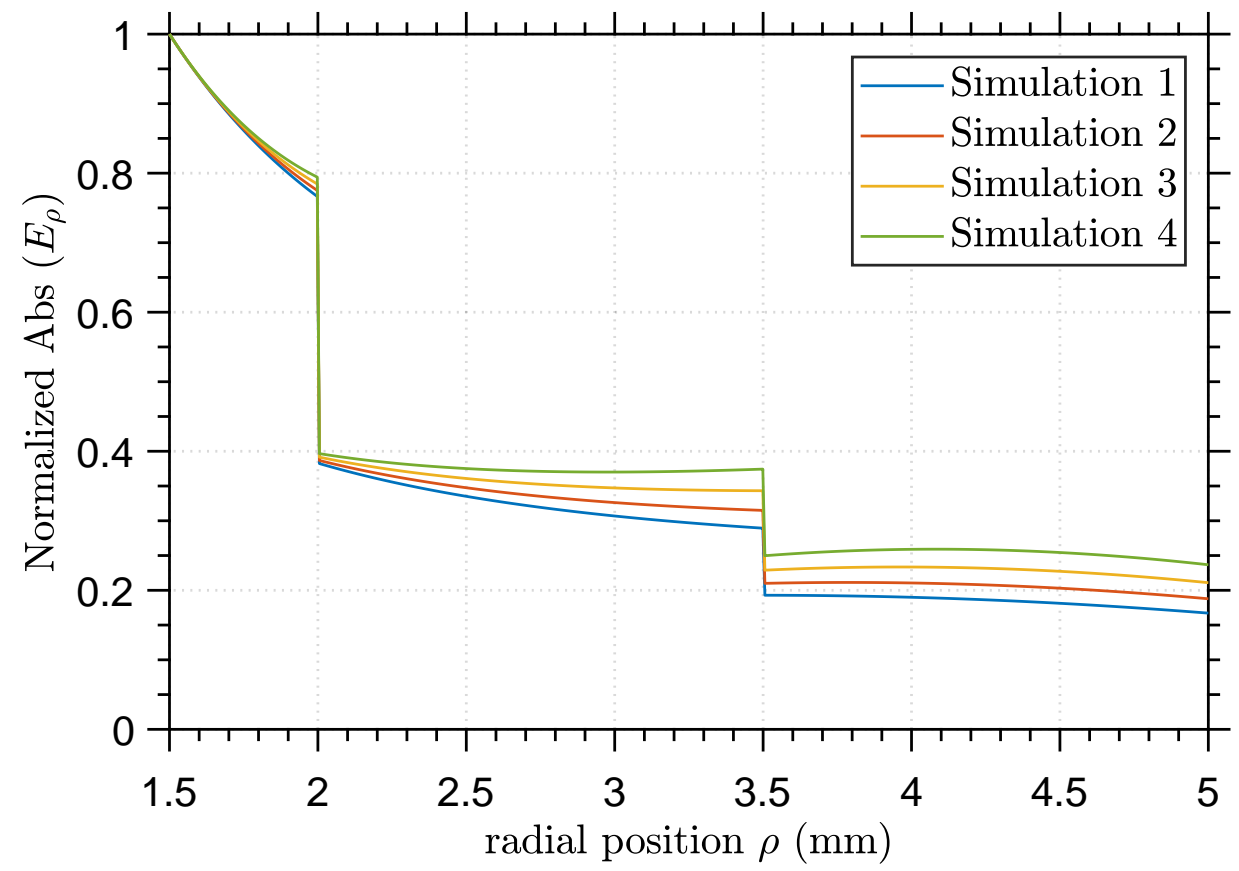

Figure 2.25: Normalized $E_{\rho}$ field component of the main propagating mode in waveguide 5 at $20 \mathrm{GHz}$. The value of $\epsilon_{r}$ were varied according to Table 2.11, to simulate different levels of anisotropy. 


\section{Mode-Matching Method for Cylindrically-Layered Waveguides}

The study of waveguide discontinuities is a relevant topic in microwave engineering. Some uses of these discontinuities are in broad-band measurement cells for the complex permittivity and permeability of a sample material [35, 39, $47,48]$. In addition, the junctions of waveguides can also be used for the design of transformers, filters, couplers, among other guided devices. Waveguide junctions are widely explored and several works have studied discontinuities over the years using different techniques. The majority of then are limited to the modeling hollow homogeneous waveguides.

Transmission lines, and therefore waveguides, discontinuities are voltagecurrent discontinuities. Then, an equivalent circuit network of the junction may be applied to solve it. Equivalent lumped circuit elements can represent a discontinuity by the admittances (or impedances) matching.

Other specialized approaches, however, are needed for dealing with multimode problems. In [70], a periodic axially symmetrical structure made by junctions of waveguides was studied using FEM. The analysis of axi-symmetric body-of-revolution (BOR) is used to simplify the study of guided structures. The FEM is also a popular method for modeling connectors and two-port junctions $[41,42]$.

A hybrid numerical method was developed in [71] to study a discontinuity in coaxial cable partially filled by a dielectric layer. Expressions for the volume integral equations associated with the Moment Method were developed in [21] to model the scattering problem of generalized anisotropic materials and in [72], the integral equation method is used in a rectangular-cross-section structure. The projection method was exploited in $[30,31]$ to compute the propagation characteristics inside a cylindrical anisotropic metal-dielectric waveguide, and the FDTD was a modeling technique for waveguide problems with complex media in $[4,26]$.

These numerical techniques are widely used and can deal with complex geometries, however, a high-cost in terms of computational resources is demanded. As an alternative, more reliable approaches based on semi-analytic methods should be explored if computational efficiency is desired. 
In the mode-matching method (MMT), the eigenmodes of each region are matched to each other at the junction discontinuity to satisfy the boundary conditions [56]. This method can be used to analyze structures with simple geometry and, many times, closed-form solutions can be derived. Another very popular MMT-based approach used to analyze inhomogeneous guided structures is the numerical mode-matching (NMM) [22, 23, 63, 64, 73-75]. In the NMM, the eigenfield solutions are calculated via a numerical FD or FE approach, and they are combined with a closed-form mode-matching boundary enforcement. In addition, it is also worth mentioning the recently introduced spectral numeric mode-matching (SNMM) technique [9], specialized for planarly-layered media. In [18], a full-wave equivalent circuit was used to model a multilayered structure with uniaxial anisotropy, and a discrete modematching (DMM) approach was then applied to solve a large system of linear equations.

In this chapter, we will use an MMT-based solution using the closedform eigenfields obtained in Chapter 2. Our approach will generalize the methodology introduced in $[25,32,33,51]$.

In order to analyze waveguide discontinuities, the generalized scattering matrix (GSM) representation will be employed for capturing the coupling between the sections of waveguides. This sub-domain decomposition allows us to obtain a low-cost computational method via a robust fashion. This robustness is explained because the associated GSM matrices are inherently stable. Other types of matrices coupling approaches, such as admittance, impedance, and transmission ones, might be unstable in some situations [76].

The MMT will enable us to determine the GSM of an entire structure by cascading the GSM matrices of each junction. We will employ here the conservation of reaction of the fields in the mode-matching enforcing, and after a series of mathematical simplifications, we will be able to present closed-form coupling coefficients in terms of Lommel integrals.

Typical applications of the MMT include analysis of waveguide circuits, microstrip circuits in conjunction with the microstrip waveguide model and integrated-circuit horn-antennas [77]. In this chapter, our MMT method will be used to analyze a series of waveguide junction problems, including a) the coupling between two homogeneous coaxial waveguides, b) the coupling between two homogeneous circular waveguides, c) the coupling between a homogeneous coaxial and a homogeneous circular waveguides, d) the coupling between an inhomogeneous coaxial and an inhomogeneous circular waveguides, among others. Also, we present numerical results of inhomogeneous and lossy guided structures. 


\section{1}

\section{Formulation}

We will consider first a homogeneous coaxial junction, as depicted in Fig 3.1. The common aperture between the two waveguides is denoted $S_{a}$, the cross-section of the waveguide in region 1 is $S_{1}$ and in the region 2 is $S_{2}$. Also, $S_{1}=S_{a}, S_{2}=S_{a}+S_{w}$ and $S_{w}$ is the surface of an impedance wall characterized by $Z_{2 w}$. For the case in which all the walls are PEC, $Z_{2 w}$ will be zero.

The formulation developed here requires that the area of waveguide 1 be completely contained inside waveguide $2\left(S_{1} \in S_{2}\right)$, as seen in Fig 3.1. If the structure has $S_{2} \in S_{1}$, an inversion of the S-matrix will be necessary, as will be detailed in the following. The cases where one region is not entirely contained inside the other will not be addressed here, but procedures for remedy the MMT in such scenarios can be found in [33, 51].

The transversal fields from equation (2-61) can be rewritten as

$$
\begin{aligned}
\mathbf{E}_{j s} & =\sum_{n=-\infty}^{\infty} \sum_{p=1}^{\infty}\left(a_{j, n p}^{+} e^{+i k_{j z, n p} z}+a_{j, n p}^{-} e^{-i k_{j z, n p} z}\right) \mathbf{e}_{j s, n p}(\rho) e^{i n \phi} \text { and } \\
\mathbf{H}_{j s} & =\sum_{n^{\prime}=-\infty}^{\infty} \sum_{p^{\prime}=1}^{\infty}\left(a_{j, n^{\prime} p^{\prime}}^{+} e^{+i k_{j z, n^{\prime} p^{\prime}} z}-a_{j, n^{\prime} p^{\prime}}^{-} e^{-i k_{j z, n^{\prime} p^{\prime}} z}\right) \mathbf{h}_{j s, n^{\prime} p^{\prime}}(\rho) e^{i n \phi}
\end{aligned}
$$

where $j$ indicates the longitudinal region $(j=1$ or 2$)$. It is possible to express

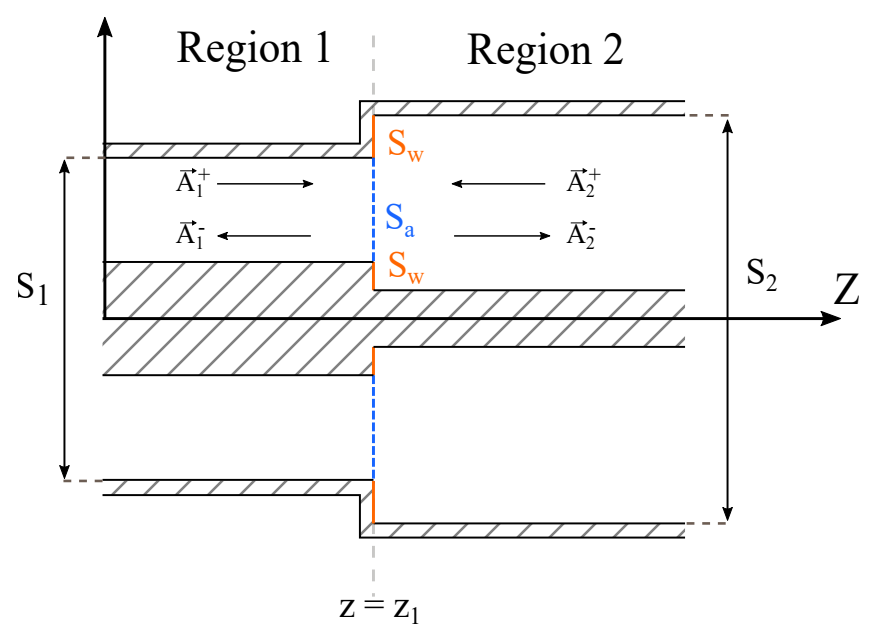

Figure 3.1: Junction of two homogeneous coaxial waveguides. 
these double sum as a simple one by means of

$$
\begin{aligned}
\mathbf{E}_{j s} & =\sum_{m}^{\infty}\left(A_{j, m}^{+}+A_{j, m}^{-}\right) \mathbf{E}_{j s, m} \text { and } \\
\mathbf{H}_{j s} & =\sum_{m^{\prime}}^{\infty}\left(A_{j, m^{\prime}}^{+}-A_{j, m^{\prime}}^{-}\right) \mathbf{H}_{j s, m^{\prime}}
\end{aligned}
$$

where $A_{j, m}^{+}$and $A_{j, m}^{-}$are the forward and backward modal amplitudes at the interface in $z=z_{1}$. These amplitudes are given by

$$
A_{j, m}^{ \pm}=a_{j, m}^{ \pm} e^{ \pm i k_{j z, m} z_{1}}
$$

At the plane $z=z_{1}$, we should impose the following boundary conditions:

$$
\begin{aligned}
& \hat{z} \times \mathbf{E}_{2 s}=\left\{\begin{array}{l}
\hat{z} \times \mathbf{E}_{1 s}, \quad \text { in } S_{a} \\
Z_{2 w} \hat{z} \times\left(\hat{z} \times \mathbf{H}_{2 s}\right), \quad \text { in } S_{w}
\end{array}\right. \\
& \hat{z} \times \mathbf{H}_{2 s}=\hat{z} \times \mathbf{H}_{1 s}, \quad \text { in } S_{a} \text {. }
\end{aligned}
$$

The waveguides support an infinite number of modes, but for numerical computation, we will truncate the summation to $M$ modes for region 1 and $N$ modes for region 2. The convergence of the modal expansion can be achieved even with small values for $M$ and $N$ as long as the truncation error caused by the high-order modes are negligible [78].

By using the vector identity

$$
-\mathbf{H}_{2 s}=\hat{z} \times\left(\hat{z} \times \mathbf{H}_{2 s}\right),
$$

and substituting equation (3-3) in (3-6), we can obtain

$$
\begin{aligned}
& \sum_{n=1}^{N}\left(A_{2, n}^{+}+A_{2, n}^{-}\right) \hat{z} \times \mathbf{E}_{2 s, n}=\sum_{m=1}^{M}\left(A_{1, m}^{+}+A_{1, m}^{-}\right) \hat{z} \times \mathbf{E}_{1 s, m}, \quad \text { in } S_{a} \\
& \sum_{n=1}^{N}\left(A_{2, n}^{+}+A_{2, n}^{-}\right) \hat{z} \times \mathbf{E}_{2 s, n}=-Z_{2 w} \sum_{n=1}^{N}\left(A_{2, n}^{+}-A_{2, n}^{-}\right) \mathbf{H}_{2 s, n}, \quad \text { in } S_{w}
\end{aligned}
$$

Taking the dot product of the above equations with $\mathbf{H}_{2 s, n^{\prime}}$, for $n^{\prime}=$ 
$1,2, \ldots N$, and integrating the result over $S_{2}$, we obtain

$$
\begin{aligned}
& \sum_{n=1}^{N}\left(A_{2, n}^{+}+A_{2, n}^{-}\right) \iint_{S_{2}}\left(\hat{z} \times \mathbf{E}_{2 s, n}\right) \cdot \mathbf{H}_{2 s, n^{\prime}} d S \\
& \quad=\sum_{m=1}^{M}\left(A_{1, m}^{+}+A_{1, m}^{-}\right) \iint_{S_{2}}\left(\hat{z} \times \mathbf{E}_{1 s, m}\right) \cdot \mathbf{H}_{2 s, n^{\prime}} d S, \text { in } S_{a} \\
& \sum_{n=1}^{N}\left(A_{2, n}^{+}+A_{2, n}^{-}\right) \iint_{S_{2}}\left(\hat{z} \times \mathbf{E}_{2 s, n}\right) \cdot \mathbf{H}_{2 s, n^{\prime}} d S \\
& \quad=-Z_{2 w} \sum_{n=1}^{N}\left(A_{2, n}^{+}-A_{2, n}^{-}\right) \iint_{S_{2}} \mathbf{H}_{2 s, n} \cdot \mathbf{H}_{2 s, n^{\prime}} d S, \text { in } S_{w} .
\end{aligned}
$$

Using the identity $(\hat{z} \times \mathbf{E}) \cdot \mathbf{H}=(\mathbf{E} \times \mathbf{H}) \cdot \hat{z}$, we obtain

$$
\begin{aligned}
\sum_{n=1}^{N}\left(A_{2, n}^{+}+A_{2, n}^{-}\right) \iint_{S_{2}}\left(\mathbf{E}_{2 s, n} \times \mathbf{H}_{2 s, n^{\prime}}\right) \cdot \hat{z} d S \\
=\sum_{m=1}^{M}\left(A_{1, m}^{+}+A_{1, m}^{-}\right) \iint_{S_{a}}\left(\mathbf{E}_{1 s, m} \times \mathbf{H}_{2 s, n^{\prime}}\right) \cdot \hat{z} d S \\
\quad-Z_{2 w} \sum_{n=1}^{N}\left(A_{2, n}^{+}-A_{2, n}^{-}\right) \iint_{S_{w}} \mathbf{H}_{2 s, n} \cdot \mathbf{H}_{2 s, n^{\prime}} d S .
\end{aligned}
$$

In the above, the second and third integrals were simplified to over $S_{a}$ and $S_{w}$ because there are no $\mathbf{E}_{1 s}$ on $S_{2}$ (only in $S_{a}$ ) and the surface impedance $Z_{2 w}$ exists only in $S_{w}$.

These equations can be written in the following matrix form:

$$
\overline{\bar{Q}}_{2}\left(\bar{A}_{2}^{+}+\bar{A}_{2}^{-}\right)=\overline{\bar{X}}_{12}\left(\bar{A}_{1}^{+}+\bar{A}_{1}^{-}\right)+\overline{\bar{L}}_{2}\left(\bar{A}_{2}^{+}-\bar{A}_{2}^{-}\right)
$$

where

$$
\begin{aligned}
& \bar{A}_{j}^{ \pm}=\left[\begin{array}{c}
A_{j, 1}^{ \pm} \\
A_{j, 2}^{ \pm} \\
\vdots
\end{array}\right], \\
& \left.\overline{\bar{X}}_{i j}\right|_{n, m}=X_{i m, j n} \text {, } \\
& \left.\overline{\bar{Q}}_{i}\right|_{n, m}=X_{i m, i n}, \\
& \left.\overline{\bar{L}}_{i}\right|_{n, m}=L_{i m, i n} .
\end{aligned}
$$

The coupling coefficient $X_{i m, j n}$ can be physically interpreted as the reaction of the $m$ th modal field in region $i$ with respect to $n$th modal field in region $j[51,78]$. This coefficient is given by

$$
X_{i m, j n}=\left\langle\mathbf{E}_{i s, m}, \mathbf{H}_{j s, n}\right\rangle=\iint_{S_{i}}\left(\mathbf{E}_{i s, m} \times \mathbf{H}_{j s, n}\right) \cdot \hat{z} \rho d \rho d \phi .
$$

In addition, $L_{i m, i n}$ is given by 


$$
L_{i m, i n}=-Z_{j w} \iint_{S_{j w}}\left(\mathbf{H}_{j s, m} \cdot \mathbf{H}_{j s, n}\right) \rho d \rho d \phi .
$$

Using equation (3-7) and repeating the same procedure as above, its possible to derive a integral equation for the magnetic field coupling:

$$
\sum_{n=1}^{N}\left(A_{2, n}^{+}-A_{2, n}^{-}\right) \hat{z} \times \mathbf{H}_{2 s, n}=\sum_{m=1}^{M}\left(A_{1, m}^{+}-A_{1, m}^{-}\right) \hat{z} \times \mathbf{H}_{1 s, m}, \quad \text { in } S_{a} .
$$

Taking the dot product with $\mathbf{E}_{1 s, m^{\prime}}$, for $m^{\prime}=1,2, \ldots, M$ and integrating over $S_{1}$, we obtain

$$
\begin{aligned}
& \sum_{n=1}^{N}\left(A_{2, n}^{+}-A_{2, n}^{-}\right) \iint_{S_{1}}\left(\mathbf{E}_{1 s, m^{\prime}} \times \mathbf{H}_{2 s, n}\right) \cdot \hat{z} d S= \\
& \sum_{m=1}^{M}\left(A_{1, m}^{+}-A_{1, m}^{-}\right) \iint_{S_{1}}\left(\mathbf{E}_{1 s, m^{\prime}} \times \mathbf{H}_{2 s, m}\right) \cdot \hat{z} d S .
\end{aligned}
$$

In a matrix form, we can write

$$
\overline{\bar{X}}_{12}^{t}\left(\bar{A}_{2}^{+}-\bar{A}_{2}^{-}\right)=\overline{\bar{Q}}_{1}\left(\bar{A}_{1}^{+}-\bar{A}_{1}^{-}\right),
$$

in which the superscript $t$ indicates the transpose. We can relate the forward and backward amplitudes combining equations (3-14) and (3-23) via a GSM matrix

$$
\left[\begin{array}{c}
\bar{A}_{1}^{-} \\
\bar{A}_{2}^{+}
\end{array}\right]=\left[\begin{array}{cc}
\overline{\bar{S}}_{11} & \overline{\bar{S}}_{12} \\
\overline{\bar{S}}_{21} & \overline{\bar{S}}_{22}
\end{array}\right]\left[\begin{array}{c}
\bar{A}_{1}^{+} \\
\bar{A}_{2}^{-}
\end{array}\right]
$$

where the scattering sub-matrices are

$$
\begin{aligned}
& \overline{\bar{S}}_{11}=\left[\overline{\bar{Q}}_{1}+\overline{\bar{X}}_{12}^{t}\left(\overline{\bar{Q}}_{2}-\overline{\bar{L}}_{2}\right)^{-1} \overline{\bar{X}}_{12}\right]^{-1}\left[\overline{\bar{Q}}_{1}-\overline{\bar{X}}_{12}^{t}\left(\overline{\bar{Q}}_{2}-\overline{\bar{L}}_{2}\right)^{-1} \overline{\bar{X}}_{12}\right], \\
& \overline{\bar{S}}_{12}=2\left[\overline{\bar{Q}}_{1}+\overline{\bar{X}}_{12}^{t}\left(\overline{\bar{Q}}_{2}-\overline{\bar{L}}_{2}\right)^{-1} \overline{\bar{X}}_{12}\right]^{-1} \overline{\bar{X}}_{12}^{t}\left[\overline{\bar{I}}+\left(\overline{\bar{Q}}_{2}-\overline{\bar{L}}_{2}\right)^{-1} \overline{\bar{L}}_{2}\right] \\
& \overline{\bar{S}}_{21}=2\left(\overline{\bar{Q}}_{2}-\overline{\bar{L}}_{2}+\overline{\bar{X}}_{12} \overline{\bar{Q}}_{1}^{-1} \overline{\bar{X}}_{12}^{t}\right)^{-1} \overline{\bar{X}}_{12}, \\
& \overline{\bar{S}}_{22}=-\left(\overline{\bar{Q}}_{2}-\overline{\bar{L}}_{2}+\overline{\bar{X}}_{12} \overline{\bar{Q}}_{1}^{-1} \overline{\bar{X}}_{12}^{t}\right)^{-1}\left(\overline{\bar{Q}}_{2}+\overline{\bar{L}}_{2}-\overline{\bar{X}}_{12} \overline{\bar{Q}}_{1}^{-1} \overline{\bar{X}}_{12}^{t}\right) .
\end{aligned}
$$

The $\overline{\bar{S}}_{i i}$ matrix, for $i=\{1,2\}$, relates the reflected amplitudes from region $i$ due to incident fields in this region, while matrix $\overline{\bar{S}}_{i j}$, for $j=\{2,1\}$, relates reflected amplitudes from region $i$, due to incident fields from region $j$.

\section{2}

\section{Reaction Integrals}

The electromagnetic reaction concept $[57,79]$ can be employed to simplify the formulation and the resolution of boundary-value problems. In our formulation, reaction integrals have appeared in (3-19). The quantity $\left\langle\mathbf{E}_{i s, m}, \mathbf{H}_{j s, n}\right\rangle$ is the the reaction between $\mathbf{E}_{i s, m}$ and $\mathbf{H}_{j s, n}$, and this can be 
understood as a physical measurable capacity of coupling between such fields. This concept can be extended to anisotropic media by using a more general form of the reciprocity theorem, as have been demonstrated in [80]. When the both permittivity and permeability tensors are transpose symmetric (as in uniaxial and biaxial anisotropic media), the conventional forms of the reciprocity theorem still apply.

Rewriting (3-19) with the modal double-index form, we have

$$
X_{i n p, j n^{\prime} p^{\prime}}=\iint_{S_{i}}\left(\mathbf{E}_{i s, n p} \times \mathbf{H}_{j s, n^{\prime} p^{\prime}}\right) \cdot \hat{z} \rho d \rho d \phi,
$$

which can be manipulated, and reduced to [51]

$$
\begin{aligned}
X_{1,2}=-(-1)^{n} \pi[ & -s_{n} \omega \epsilon_{2 s} k_{1 z} \alpha_{1}^{e} \alpha_{2}^{e} \hat{\mathcal{L}}_{n}^{+}\left(B_{n}^{e 1}, B_{n}^{e 2}\right)+\omega \mu_{1 s} k_{2 z} \alpha_{1}^{h} \alpha_{2}^{h} \hat{\mathcal{L}}_{n}^{+}\left(B_{n}^{h 1}, B_{n}^{h 2}\right) \\
& \left.-i k_{1 z} k_{2 z} \alpha_{1}^{e} \alpha_{2}^{h} \hat{\mathcal{L}}_{n}^{-}\left(B_{n}^{e 1}, B_{n}^{h 2}\right)-i \omega^{2} \epsilon_{2 s} \mu_{1 s} \alpha_{2}^{e} \alpha_{1}^{h} \hat{\mathcal{L}}_{n}^{-}\left(B_{n}^{h 1}, B_{n}^{e 2}\right)\right] .
\end{aligned}
$$

In the above, subscripts $n p$ were omitted for shorting our notation, $s_{n}=$ $1-2 \delta_{0, n}, B_{n}^{e, h j}$ are combinations of cylindrical functions $(j=1$ or 2$), \hat{\mathcal{L}}_{n}^{ \pm}$ are the coupling integrals

$$
\hat{\mathcal{L}}_{n}^{ \pm}\left(B_{n}^{1}, B_{n}^{2}\right)=\frac{1}{k_{1 \rho} k_{2 \rho}} \mathcal{L}_{n}^{ \pm}\left(B_{n}^{1}, B_{n}^{2}\right)
$$

with

$$
\mathcal{L}_{n}^{ \pm}\left(B_{n}^{1}, B_{n}^{2}\right)=\left[\mathcal{L}_{n-1}\left(B_{n-1}^{1}, B_{n-1}^{2}\right) \pm \mathcal{L}_{n+1}\left(B_{n+1}^{1}, B_{n+1}^{2}\right)\right],
$$

where the Lommel integrals are given by

$$
\mathcal{L}_{m}\left(B_{m}^{1}, B_{m}^{2}\right)=\int_{\rho_{\min }}^{\rho_{\max }} B_{m}^{1}\left(\alpha_{1} k_{1 \rho} \rho\right) B_{m}^{2}\left(\alpha_{2} k_{2 \rho} \rho\right) \rho d \rho .
$$

A detailed analytical formulations of the above Lommel integrals were presented in [51, 63]. Some important results are summarized here. Expression in (3-30) can be separated in four parts:

$$
X_{1,2}=X_{1,2}^{e e}+X_{1,2}^{h h}+X_{1,2}^{e h}+X_{1,2}^{h e} .
$$

where superscripts $e$ and $h$ are associated to the axial $E_{z}$ and $H_{z}$ field components.

For the case in which $\alpha_{1} k_{1 \rho} \neq \alpha_{2} k_{2 \rho}$, the Lommel integral becomes

$$
\begin{array}{r}
\int_{\rho_{\text {min }}}^{\rho_{\max }} B_{m}^{1}\left(\alpha_{1} k_{1 \rho} \rho\right) B_{m}^{2}\left(\alpha_{2} k_{2 \rho} \rho\right) \rho d \rho=\left[\frac{\rho}{\left(\alpha_{1} k_{1 \rho}\right)^{2}-\left(\alpha_{2} k_{2 \rho}\right)^{2}}\right. \\
\left.\times\left(\alpha_{1} k_{1 \rho} B_{m+1}^{1} B_{m}^{2}-\alpha_{2} k_{2 \rho} B_{m}^{1} B_{m+1}^{2}\right)\right]_{\rho_{\min }}^{\rho_{\max }}
\end{array}
$$


which can be further simplified to

$$
\begin{aligned}
& \mathcal{L}_{m}^{ \pm}\left(B_{m}^{1}, B_{m}^{2}\right)=\left[\mp \frac{2 m}{\alpha_{1} k_{1 \rho} \alpha_{2} k_{2 \rho}} B_{m}^{1} B_{m}^{2}\right. \\
& \left.+\frac{(1 \pm 1) \rho}{\left(\alpha_{1} k_{1 \rho}\right)^{2}-\left(\alpha_{2} k_{2 \rho}\right)^{2}}\left(\alpha_{1} k_{1 \rho} B_{m}^{1} B_{m-1}^{2}-\alpha_{2} k_{2 \rho} B_{m-1}^{1} B_{m}^{2}\right)\right]_{\rho_{\min }}^{\rho_{\max }}
\end{aligned}
$$

In the case where $\alpha_{1} k_{1 \rho} \approx \alpha_{2} k_{2 \rho}$, the expression for $\mathcal{L}_{m}^{ \pm}\left(B_{m}^{1}, B_{m}^{2}\right)$ can be reduced to

$$
\begin{array}{r}
\mathcal{L}_{m}^{ \pm}\left(B_{m}^{1}, B_{m}^{2}\right)=\left\{\frac { \rho ^ { 2 } } { 4 } \left[2 B_{m-1}^{1} B_{m-1}^{2} \pm 2 B_{m+1}^{1} B_{m+1}^{2}-B_{m}^{1}\left(B_{m-2}^{2} \pm B_{m+2}^{2}\right)\right.\right. \\
\left.\left.-B_{m}^{2}\left(B_{m-2}^{1} \pm B_{m+2}^{1}\right)\right]\right\}_{\rho_{\min }}^{\rho_{\max }}
\end{array}
$$

Also, when $\alpha_{1} k_{1 \rho}=\alpha_{2} k_{2 \rho}$, the expression in (3-33) allows us to obtain

$$
\mathcal{L}_{m}^{ \pm}\left(B_{m}^{1}, B_{m}^{1}\right)=\left\{\frac{\rho^{2}}{2}\left[\left(B_{m-1}^{1}\right)^{2} \pm\left(B_{m+1}^{1}\right)^{2}-\left(B_{m-2}^{1} B_{m}^{1} \pm B_{m}^{1} B_{m+2}^{1}\right)\right]\right\}_{\rho_{\min }}^{\rho_{\max }}
$$

\subsection{1}

\section{Solutions for the TEM-mode}

We will discuss now a particular case that may occur when analyzing waveguide at junctions, where the TEM mode is supported. This propagating mode will exist only in homogeneous coaxial waveguides for the azimuthal symmetric harmonic $n=0$. The TEM is characterized by $k_{\rho}=0$, which may lead to a numerical issues when using the closed-form solutions of the Lommel integrals or when using (3-30). However, as the cylindrical functions $B_{n}^{e, h j}$ presents $k_{\rho}$ in its arguments, it becomes possible use a limiting evaluations on the expressions (3-36) to (3-38) to avoid singularities.

The functions $B_{n}^{e, h j}$ are combinations of Bessel functions of integer order $n$, in the form

$$
B_{n}(x)=a H_{n}(x)+b J_{n}(x),
$$

Using small argument approximations for the cylindrical functions [1, 67], we obtain

for $n=0$, and

$$
B_{0}\left(\alpha_{j} k_{j \rho} \rho\right) \simeq a i\left(\frac{2}{\pi}\right) \ln \left(\alpha_{j} k_{j \rho} \rho\right)
$$

$$
B_{n}\left(\alpha_{j} k_{j \rho} \rho\right) \simeq a(-i) \frac{(n-1) !}{\pi}\left(\frac{2}{\alpha_{j} k_{j \rho} \rho}\right)^{n}
$$

and for $n>0$. 
As $H_{n}(\cdot)$ dominates $J_{n}(\cdot)$ when $k_{\rho} \longrightarrow 0$, we only need to evaluate the Hankel functions in this limiting form. The amplitude $a$ is obtained from the field equations. In TEM mode, will exist only $e_{\rho}$ and $h_{\phi}$ components, then, by using the set of equations (2-33)-(2-36), we obtain

$$
\begin{aligned}
e_{j \rho} & =\frac{1}{k_{j \rho}^{2} \rho}\left(i k_{j z} \alpha_{j}^{e} k_{j \rho} \rho B_{n}^{\prime e j}-n \omega \mu_{j s} B_{n}^{h j}\right), \\
h_{j \phi} & =\frac{1}{k_{j \rho}^{2} \rho}\left(i \omega \epsilon_{j s} \alpha_{j}^{e} k_{j \rho} \rho B_{n}^{\prime e j}-n k_{j z} B_{n}^{h j}\right)
\end{aligned}
$$

with $j=1$ or 2 . For $n=0$, the $e_{j \rho}$ component becomes

$$
e_{j \rho}=\frac{1}{k_{j \rho}^{2} \rho}\left(i k_{j z} \alpha_{j}^{e} k_{j \rho} \rho B_{0}^{\prime e j}\left(\alpha_{j}^{e} k_{j \rho} \rho\right)\right)
$$

By using the identity $H_{0}^{\prime}(x)=-H_{1}(x)[67]$

$$
e_{j \rho}=\frac{1}{k_{j \rho}^{2} \rho}\left[i k_{j z} \alpha_{j}^{e} k_{j \rho} \rho\left(-H_{1}^{e j}\left(\alpha_{j}^{e} k_{j \rho} \rho\right)\right)\right]
$$

using (3-41) in (3-45)

$$
\begin{aligned}
e_{j \rho} & =\frac{1}{k_{j \rho}^{2} \rho}\left[i k_{j z} \alpha_{j}^{e} k_{j \rho} \rho\left(\frac{2 i a}{\pi \alpha_{j}^{e} k_{j \rho} \rho}\right)\right] \\
& =\frac{-2 k_{j z} a}{k_{j \rho}^{2} \pi \rho}
\end{aligned}
$$

As the field of a TEM mode must be finite, the amplitude $a$ has to be proportional to $k_{j \rho}^{2}$. The same result is obtained using the expression for $h_{j \phi}$. As a result, the closed-form solutions for the Lommel integrals should employ the limiting forms listed bellow:

$$
\begin{aligned}
& B_{0}^{j} \longrightarrow k_{j \rho}^{2} \frac{2 i}{\pi} \ln \left(\alpha_{j} k_{j \rho} \rho\right), \\
& B_{1}^{j} \longrightarrow-k_{j \rho} \frac{2 i}{\pi \alpha_{j} \rho}, \\
& B_{2}^{j} \longrightarrow \frac{-4 i}{\pi \alpha_{j}^{2} \rho^{2}} .
\end{aligned}
$$

When the order $n$ is an integer, we can use $B_{-n}(x)=(-1)^{n} B_{n}(x)[1]$. 
Then, it is useful to write

$$
\begin{aligned}
& B_{0}^{j} \longrightarrow k_{j \rho}^{2} \frac{2 i}{\pi} \ln \left(\alpha_{j} k_{j \rho} \rho\right), \\
& B_{ \pm 1}^{j} \longrightarrow \mp k_{j \rho} \frac{2 i}{\pi \alpha_{j} \rho}, \\
& B_{ \pm 2}^{j} \longrightarrow \frac{-4 i}{\pi \alpha_{j}^{2} \rho^{2}}
\end{aligned}
$$

The TEM mode will occur in a inhomogeneous to homogeneous (and vice-versa) or in a homogeneous to homogeneous junction, as shown in Fig 3.2.

For the homogeneous to inhomogeneus case (Fig 3.2a), where the TEM mode exists only in region $1\left(k_{1 \rho}=0\right)$ and therefore, $\alpha_{1} k_{1 \rho} \neq \alpha_{2} k_{2 \rho}$, the

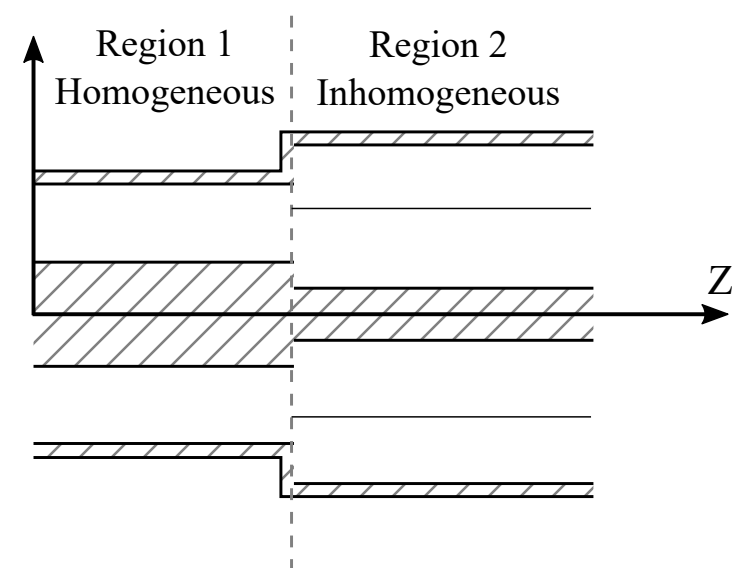

(a)

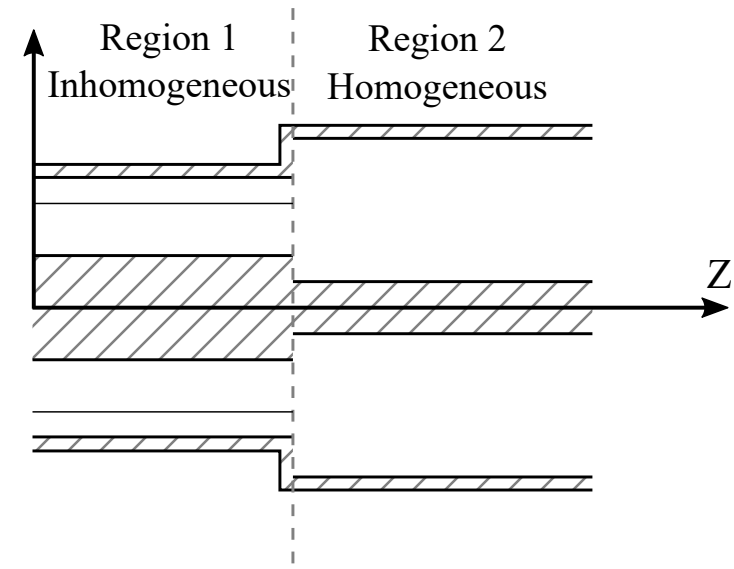

(b)

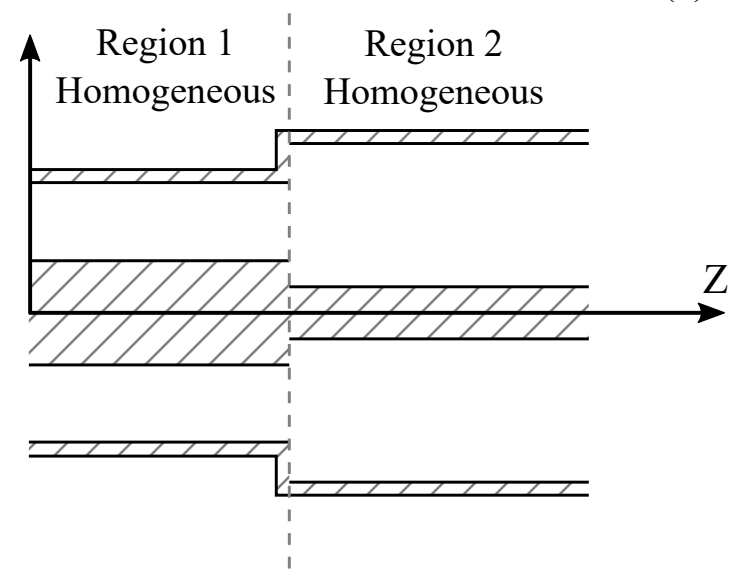

(c)

Figure 3.2: Waveguide discontinuity cases where the TEM mode occur: (a) homogeneous to inhomogeneous junction, (b) inhomogeneous to homogeneous junction and (c) homogeneous to homogeneous junction. 
expression in (3-36) will become to

$$
\begin{aligned}
& \mathcal{L}_{0}^{+}\left(B_{0}^{1}, B_{0}^{2}\right)=\left[\frac{2 \rho}{\left(\alpha_{1} k_{1 \rho}\right)^{2}-\left(\alpha_{2} k_{2 \rho}\right)^{2}}\left(\alpha_{1} k_{1 \rho} B_{0}^{1} B_{-1}^{2}-\alpha_{2} k_{2 \rho} B_{-1}^{1} B_{0}^{2}\right)\right]_{\rho_{\min }}^{\rho_{\max }} \\
& \mathcal{L}_{0}^{-}\left(B_{0}^{1}, B_{0}^{2}\right)=0
\end{aligned}
$$

Using the approximations in the $\hat{\mathcal{L}}_{0}^{+}$expression

$$
\begin{array}{r}
\lim _{k_{1 \rho} \longrightarrow 0} \hat{\mathcal{L}}_{0}^{+}=\frac{1}{k_{1 \rho} k_{2 \rho}} \frac{2 \rho}{\left(\alpha_{1} k_{1 \rho}\right)^{2}-\left(\alpha_{2} k_{2 \rho}\right)^{2}}\left(\alpha_{1} k_{1 \rho} k_{1 \rho}^{2} \frac{2 i}{\pi} \ln \left(\alpha_{1} k_{1 \rho} \rho\right) B_{-1}^{2}\right. \\
\left.-\alpha_{2} k_{2 \rho} k_{1 \rho} \frac{2 i}{\pi \alpha_{1} \rho} B_{0}^{2}\right)
\end{array}
$$

which can be simplified to

$$
\lim _{k_{1 \rho} \longrightarrow 0} \hat{\mathcal{L}}_{0}^{+}=\left[\frac{4 i}{k_{2 \rho}^{2} \alpha_{1} \alpha_{2} \pi} B_{0}^{2}\right]_{\rho_{\min }}^{\rho_{\max }} \quad, \quad \lim _{k_{\rho} \longrightarrow 0} \hat{\mathcal{L}}_{0}^{-}=0 .
$$

In the inverse case (Fig. 3.2b), with $k_{2 \rho}=0$, the result would be

$$
\lim _{k_{2 \rho} \longrightarrow 0} \hat{\mathcal{L}}_{0}^{+}=\left[\frac{4 i}{k_{1 \rho}^{2} \alpha_{1} \alpha_{2} \pi} B_{0}^{1}\right]_{\rho_{\min }}^{\rho_{\max }} \quad, \quad \lim _{k_{2 \rho} \longrightarrow 0} \hat{\mathcal{L}}_{0}^{-}=0
$$

For a two homogeneous waveguide junction (Fig. 3.2c), where $k_{1 \rho}=k_{2 \rho}=$ 0 and $\alpha_{1} k_{1 \rho}=\alpha_{2} k_{2 \rho}$, the $\mathcal{L}_{0}^{ \pm}$coupling integrals will be

$$
\begin{aligned}
& \mathcal{L}_{0}^{+}\left(B_{0}^{1}, B_{0}^{1}\right)=\left\{\frac{\rho^{2}}{2}\left[\left(B_{-1}^{1}\right)^{2}+\left(B_{1}^{1}\right)^{2}-\left(B_{-2}^{1} B_{0}^{1}+B_{0}^{1} B_{2}^{1}\right)\right]\right\}_{\rho_{\min }}^{\rho_{\max }} \\
& \mathcal{L}_{0}^{-}\left(B_{0}^{1}, B_{0}^{1}\right)=\left\{\frac{\rho^{2}}{2}\left[\left(B_{-1}^{1}\right)^{2}-\left(B_{1}^{1}\right)^{2}-\left(B_{-2}^{1} B_{0}^{1}-B_{0}^{1} B_{2}^{1}\right)\right]\right\}_{\rho_{\min }}^{\rho_{\max }}
\end{aligned}
$$

It is easy to notice that as $B_{-1}=-B_{1}$ and $B_{-2}=B_{2}$ the result of $\mathcal{L}_{0}^{-}\left(B_{0}^{1}, B_{0}^{1}\right)$ will be zero. Then, applying the approximations in equation (3-58), $\hat{\mathcal{L}}_{0}^{+}$will be

$$
\lim _{k_{1 \rho} \longrightarrow 0} \hat{\mathcal{L}}_{0}^{+}=\frac{1}{k_{1 \rho}^{2}} \frac{\rho^{2}}{2}\left[2\left(k_{1 \rho} \frac{2 i}{\pi \alpha_{1} \rho}\right)^{2}-2\left(\frac{-4 i}{\pi \alpha_{1}^{2} \rho^{2}} k_{1 \rho}^{2} \frac{2 i}{\pi} \ln \left(\alpha_{1} k_{1 \rho} \rho\right)\right)\right]
$$

and after a few simplifications

$$
\lim _{k_{1 \rho} \longrightarrow 0} \hat{\mathcal{L}}_{0}^{+}=\frac{4}{\pi^{2} \alpha_{1}^{2}}\left[-1+2 \ln \left(\alpha_{1} k_{1 \rho} \rho\right)\right]_{\rho_{\min }}^{\rho_{\max }} .
$$

Evaluating the integral limits, we notice that the first term of the sum will not contribute. Using the $\log$ properties, the $\left(\alpha_{1} k_{1 \rho}\right)$ argument, which could create numerical problems, can be also eliminated. Therefore, 
the approximations arrive to

$$
\lim _{k_{1 \rho} \longrightarrow 0} \hat{\mathcal{L}}_{0}^{+}=\frac{8}{\pi^{2} \alpha_{1}^{2}} \ln \left(\frac{\rho_{\text {max }}}{\rho_{\min }}\right) \quad, \quad \lim _{k_{1 \rho} \longrightarrow 0} \hat{\mathcal{L}}_{0}^{-}=0 .
$$

It is possible to observe that when we have the TEM mode, the expression (3-34) will reduce to $X_{1,2}=X_{1,2}^{e e}$, since $X_{1,2}^{e h}=X_{1,2}^{h e}=0$, as all $\hat{\mathcal{L}}_{0}^{-}=0$, and $X_{1,2}^{h h}$ will also be zero because there are only the $e_{1 \rho}$ and $h_{2 \phi}$ components of the fields.

\subsection{2}

\section{Regions with Arbitrary Layers}

If our junction is formed by regions with non-homogeneous media, we should generalize the above-mentioned MMT formulations. Consider the coupling between a waveguide with $N_{1}$ layers in region 1 and $N_{2}$ layers in region 2 . The set of radius of region 2 that intercept the coupling aperture $S_{a}$, shown in Fig 3.3, will be called $r_{12}$.

Let $r_{i}$ be the set of radius of region $i$, for $i=1$ or 2 . The set $r_{a}$ is the sorted union between $r_{12}$ and $r_{1}$. In summary:

$$
\begin{aligned}
& r_{1}=\left\{\rho_{0}, \rho_{1}, \ldots, \rho_{N_{1}}\right\}, \\
& r_{2}=\left\{\rho_{0}, \rho_{1}, \ldots, \rho_{N_{2}}\right\}, \\
& r_{a}=\operatorname{sort}\left(r_{1} \cup r_{12}\right) .
\end{aligned}
$$

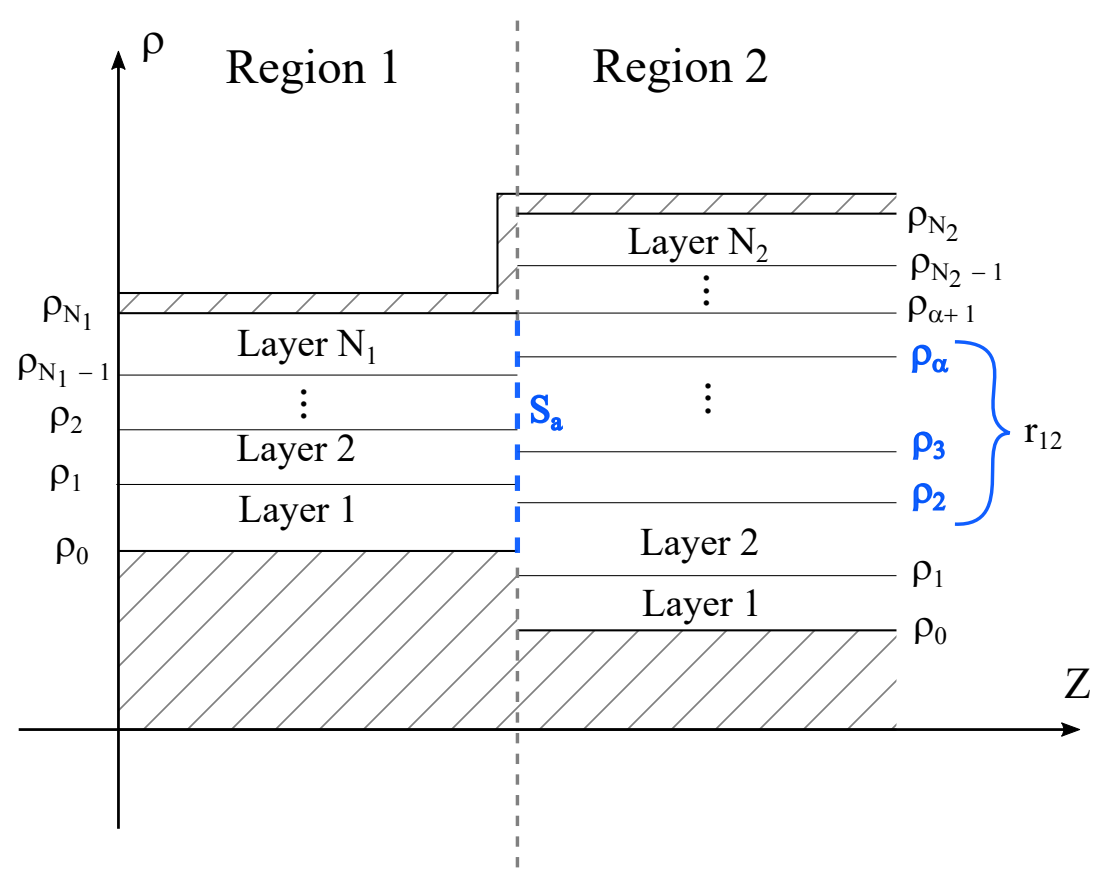

Figure 3.3: Junction between waveguides with non-homogeneous media. 
Then, the reaction integral for the inhomogeneous problem can employ our results for homogeneous coupling, resulting in [51]

$$
\begin{aligned}
& X_{1(n p), 2\left(n p^{\prime}\right)}=-2 \pi(-1)^{n} \sum_{j=0}^{\operatorname{dim}\left(r_{a}\right)} \int_{r_{a, j}}^{r_{a, j+1}}\left[s_{n} e_{1 \rho, n p}(\rho) h_{2 \phi, n p^{\prime}}(\rho)\right. \\
& \left.+e_{1 \phi, n p}(\rho) h_{2 \rho, n p^{\prime}}(\rho)\right] \rho d \rho
\end{aligned}
$$

and

$$
\begin{array}{r}
Q_{i, n p}=-2 \pi(-1)^{n} \sum_{j=0}^{\operatorname{dim}\left(r_{i}\right)} \int_{r_{i, j}}^{r_{i, j+1}}\left[s_{n} e_{i \rho, n p}(\rho) h_{i \phi, n p}(\rho)\right. \\
\left.+e_{i \phi, n p}(\rho) h_{i \rho, n p}(\rho)\right] \rho d \rho
\end{array}
$$

for $j=1,2$. As in the homogeneous scenarios, close-form expressions can trivially obtained for $X_{1(n p), 2\left(n p^{\prime}\right)}$ and $Q_{i, n p}$.

\section{3}

\section{Cascading Generalized Scattering Matrices}

If our problem has more than one junction, as in Fig 3.4, the GSM is obtained by cascading the S-matrix of each discontinuity.

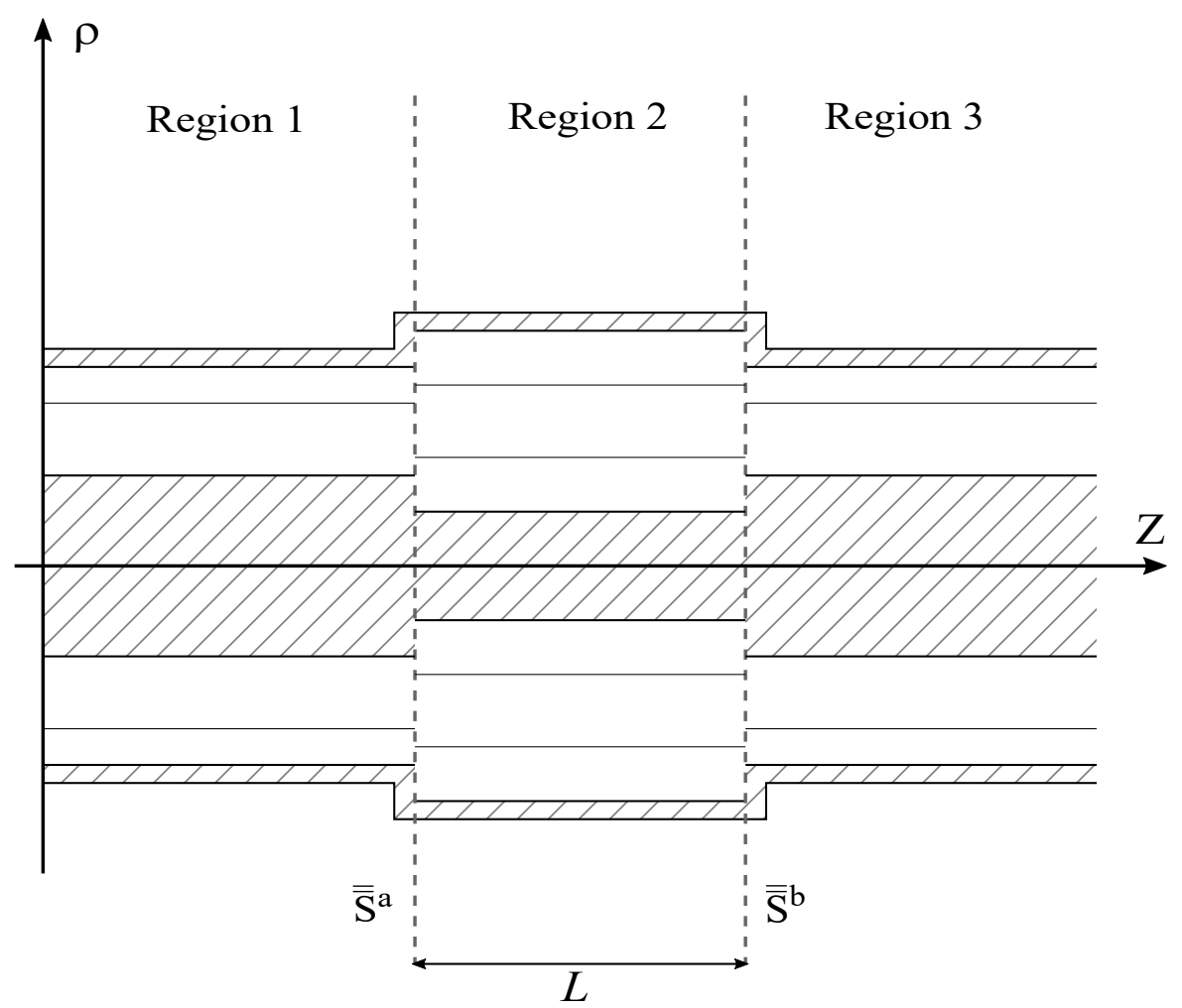

Figure 3.4: Structure with three regions. 
If the first junction is characterized by the matrix $\overline{\bar{S}}^{a}$ and the second junction by $\overline{\bar{S}}^{b}$, the GSM of the complete problem can be written as $[51,63,64]$

where

$$
\overline{\bar{S}}=\left[\begin{array}{cc}
\overline{\bar{S}}_{11}^{c} & \overline{\bar{S}}_{12}^{c} \\
\overline{\bar{S}}_{21}^{c} & \overline{\bar{S}}_{22}^{c}
\end{array}\right],
$$

$$
\begin{aligned}
& \overline{\bar{S}}_{11}^{c}=\overline{\bar{S}}_{12}^{a}\left(\overline{\bar{I}}-\overline{\bar{S}}_{11}^{b} \overline{\bar{S}}_{22}^{a}\right)^{-1} \overline{\bar{S}}_{11}^{b} \overline{\bar{S}}_{21}^{a}+\overline{\bar{S}}_{11}^{a}, \\
& \overline{\bar{S}}_{12}^{c}=\overline{\bar{S}}_{12}^{a}\left(\overline{\bar{I}}-\overline{\bar{S}}_{11}^{b} \overline{\bar{S}}_{22}^{a}\right)^{-1} \overline{\bar{S}}_{12}^{b}, \\
& \overline{\bar{S}}_{21}^{c}=\overline{\bar{S}}_{21}^{b}\left(\overline{\bar{I}}-\overline{\bar{S}}_{22}^{a} \overline{\bar{S}}_{11}^{b}\right)^{-1} \overline{\bar{S}}_{21}^{a}, \\
& \overline{\bar{S}}_{22}^{c}=\overline{\bar{S}}_{21}^{b}\left(\overline{\bar{I}}-\overline{\bar{S}}_{22}^{a} \overline{\bar{S}}_{11}^{b}\right)^{-1} \overline{\bar{S}}_{22}^{a} \overline{\bar{S}}_{12}^{b}+\overline{\bar{S}}_{22}^{b} .
\end{aligned}
$$

For intermediary waveguide sections, as is the case of region 2 in Fig. 3.4 , the scattering matrix is associated to the length of the guide and to the eigenvalues $k_{z}$ of that regions. The S-matrix of this type of junction is defined as [33]

$$
\overline{\bar{S}}^{g}=\left[\begin{array}{cc}
0 & \overline{\bar{S}}_{12}^{g} \\
\overline{\bar{S}} & 0
\end{array}\right],
$$

where

$$
\overline{\bar{S}}_{12}^{g}=\left[\begin{array}{cc}
e^{i k_{z, n} L} & 0 \\
0 & e^{i k_{z, n} L}
\end{array}\right],
$$

for the $n$th propagating mode and a guide with length $L$. Here, the exponential is positive, while in [33] is negative. This is due to the fact that in this work we adopt the time-harmonic dependence in the form $e^{-i \omega t}$ while the reference used $e^{+\sqrt{-1} \omega t}$.

In the example of Fig 3.4, the area of region 1 is completely contained in region 2 , however, region 2 is not contained in region 3 . As mentioned before at the beginning of this chapter, an inversion of the scattering matrix associated to this junction (given by (3-24)) will be necessary before the cascading procedure.

A normalization of the GSM will be used to make it independent of the fields amplitudes. The normalized matrix is given by [78, 81]

$$
\begin{aligned}
& \hat{\overline{\bar{S}}}_{11}^{c}=\operatorname{abs}\left(\overline{\bar{Q}}_{1}\right)^{1 / 2} \overline{\bar{S}}_{11}^{c} \operatorname{abs}\left(\overline{\bar{Q}}_{1}\right)^{-1 / 2} \\
& \hat{\bar{S}}_{12}^{c}=\operatorname{abs}\left(\overline{\bar{Q}}_{1}\right)^{1 / 2} \overline{\bar{S}}_{12}^{c} \operatorname{abs}\left(\overline{\bar{Q}}_{2}\right)^{-1 / 2} \\
& \hat{\bar{S}}_{21}^{c}=\operatorname{abs}\left(\overline{\bar{Q}}_{2}\right)^{1 / 2} \overline{\bar{S}}_{21}^{c} \operatorname{abs}\left(\overline{\bar{Q}}_{1}\right)^{-1 / 2} \\
& \hat{\overline{\bar{S}}}_{22}^{c}=\operatorname{abs}\left(\overline{\bar{Q}}_{2}\right)^{1 / 2} \overline{\bar{S}}_{22}^{c} \operatorname{abs}\left(\overline{\bar{Q}}_{2}\right)^{-1 / 2} .
\end{aligned}
$$

This normalization provides the property $\hat{\bar{S}} \hat{\bar{S}}=\overline{\bar{I}}$, which allows an 
easy way to verify if the scattering matrix was correctly implemented. The mathematical formulation presented in Chapters 2 and 3 were numerically implemented via the Matlab platform [68]. A simplified flowchart of the developed algorithms is presented in Fig. 3.5. The procedure described is repeated for all of the frequencies we wish to evaluate.

\section{4 \\ Validation}

In this section we will present results in order to validate the algorithm developed for the mode-matching method for cylindrical waveguide junctions. Comparisons with some results from [33] are made to verify our mathematical methodology and numerical algorithm. In addition, the Computer Simulation Technology Microwave Studio software (CST) [82] was used to validate some of the case studies. We will present first simple junctions with only two regions, composed of coaxial to coaxial, coaxial to circular, and circular to circular junctions. Later, structures with more regions will be evaluated.

\subsection{1 \\ Coaxial Junctions}

\section{Junction 1}

The first example is a junction of two heterogeneous waveguides with two radial layers each, as depicted in Fig 3.6, and with the parameters given in Table 3.1. In this simulation, we want to show that our algorithm can provide accurate results and analyze the modal convergence.

It is possible to observe from Fig 3.7 that our result of the reflection coefficient in decibel, i.e., $20 \log _{10}\left(\left.\overline{\bar{S}}_{11}\right|_{1,1}\right)$, as a function of the frequency for the first mode in region 1 agrees with the reference one (obtained with the author of [33]). The convergence is achieved when using 5 modes in each waveguide. We can notice that as the number of modes considered increases, the curve has better results. As mentioned in Chapter 2, the evanescent modes becomes important next to junctions, then, it is necessary to include more than one mode to secure the correctness of our analyzes. Considering five modes or more, we can guarantee that our results are accurate.

In addition, we can observe that the $S_{11}$ curve had an almost-flat behavior up to about $35 \mathrm{GHz}$. This is due to the cutoff frequency of the $\mathrm{TM}_{01}$ mode in waveguide 2, that is $35.64 \mathrm{GHz}$. Above this frequency, the $\mathrm{TM}_{01}$ modal field 


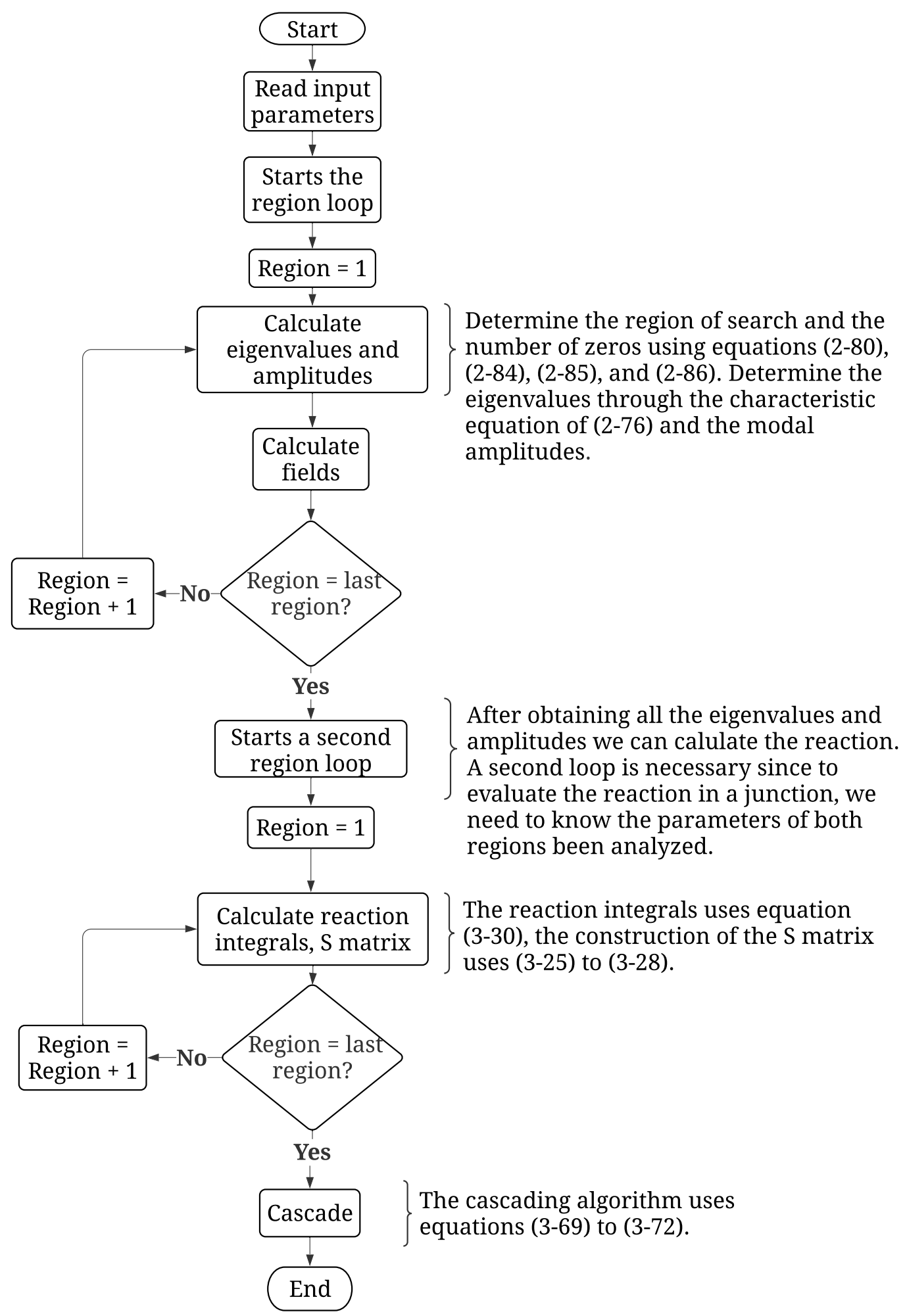

Figure 3.5: Flowchart of the developed algorithm.

becomes a propagating one, and couple with the fundamental $\mathrm{TM}_{00}$ mode of region 1.

In order to facilitate the identification of the frequency where a mode becomes propagating, we will indicate that in our figures. For example, the 
label $\mathrm{TM}_{01}^{2}$ in Fig 3.7 indicates the mode $\mathrm{TM}_{01}$ in region 2 becomes propagating at about $35 \mathrm{GHz}$.

\begin{tabular}{cccccc}
\hline Region & $\rho_{0}(\mathrm{~mm})$ & $\rho_{1}(\mathrm{~mm})$ & $\rho_{2}(\mathrm{~mm})$ & $\epsilon_{r 1}$ & $\epsilon_{r 2}$ \\
\hline 1 & 1.84 & 2.00 & 5.00 & 2.55 & 1 \\
2 & 1.84 & 2.00 & 6.00 & 2.55 & 1 \\
\hline \multicolumn{5}{c}{$\sigma=10^{-6}(\mathrm{~S} / \mathrm{m})$} \\
\hline
\end{tabular}

Table 3.1: Dimensions and parameters of junction 1.

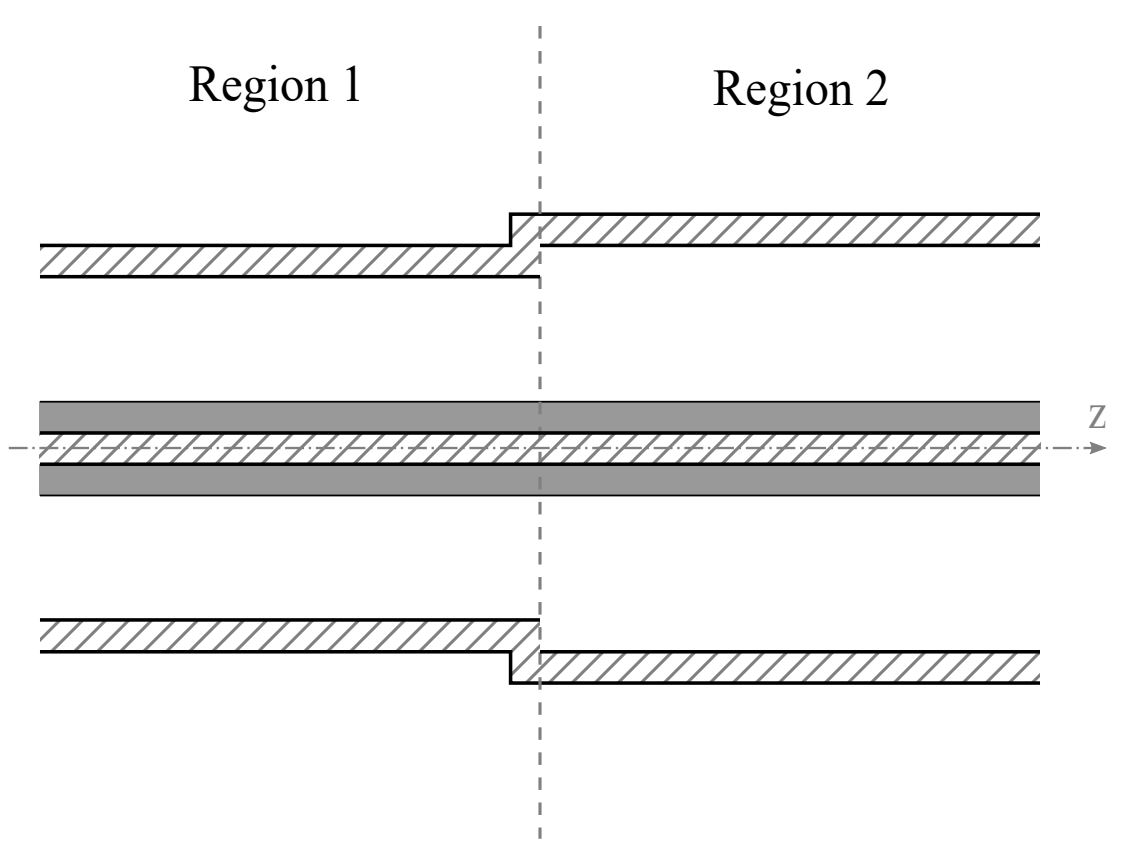

Figure 3.6: Geometry of junction 1. The darker region represents the inner layers with $\epsilon_{r}=2.55$. 


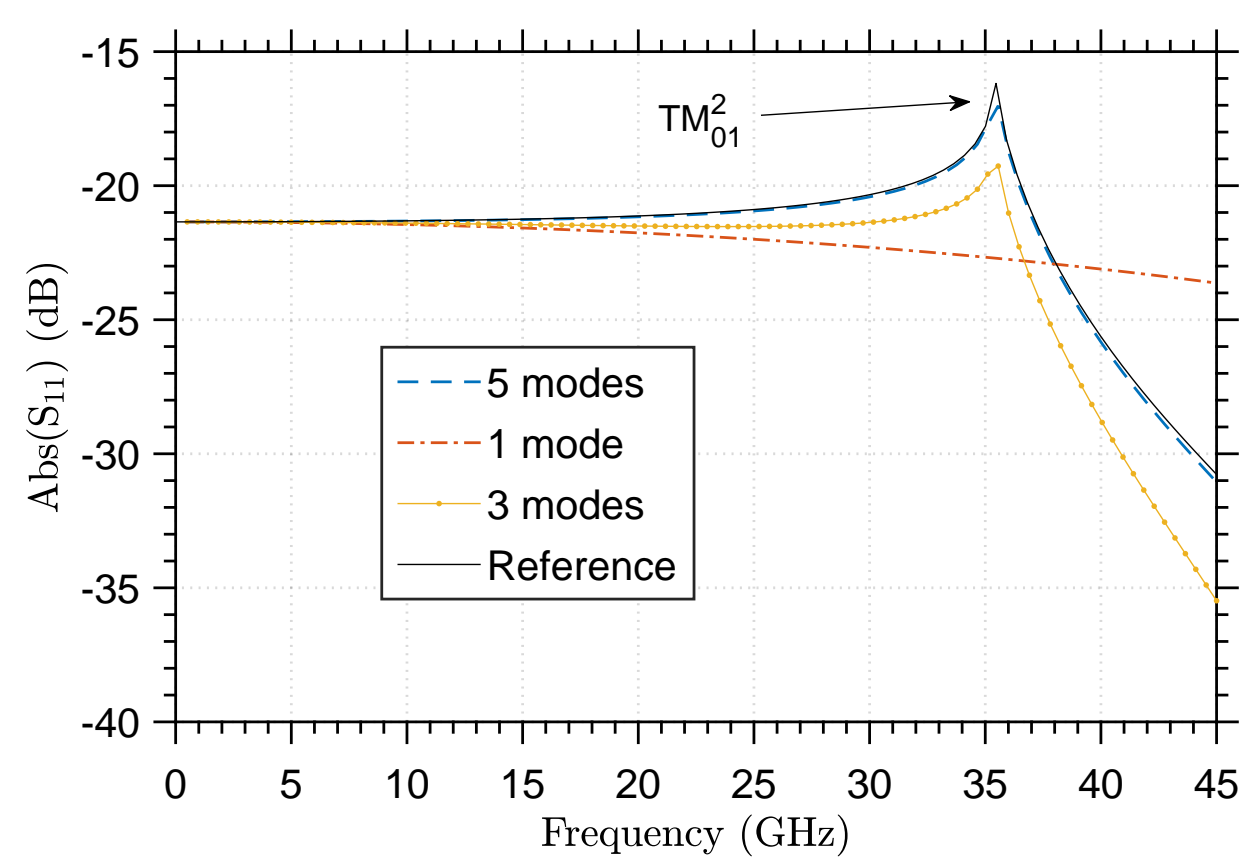

Figure 3.7: Reflection coefficient of junction 1 from 0.5 to $45 \mathrm{GHz}$.

\section{Junction 2}

The second example analyzed is a homogeneous to homogeneous junction. Considering a waveguide with two layers and sharing the same values of $\epsilon_{r}$, we will have a waveguide that is equivalent to a homogeneous one, with only one radial layer. In this case, the $k_{\rho}=0$ will be an eigenvalue solution of our characteristic equation. This will cause numerical issues in the calculation of the reaction integrals. For this reason, we will show that we can simulate such kind of homogeneous junction using waveguides with two radial layers if numerically-similar values of $\epsilon$ are employed.

Junction 2 (see the geometry in Fig. 3.8) is defined with the parameters shown in Table 3.2. Fig 3.9 present results for the reflection coefficient obtained when $\epsilon_{r 1}$ vary between $\{1.1,1.01,1.001\}$ in comparison with the reference value obtained for the same structure. It was considered 20 modes in all of the simulations. As the value of the permittivity tends to 1 , our results approximate the reference curve, which means that using layers with similar $\epsilon_{r 1}$, our problem becomes numerically-equivalent to the one of a homogeneous junction.

As we are evaluating a homogeneous coaxial waveguide, the TEM mode propagates in both regions. Above $42.57 \mathrm{GHz}$, the $\mathrm{TM}_{01}$ mode in waveguide 2 starts to propagate, and at $47 \mathrm{GHz}$ the $\mathrm{TM}_{01}$ mode in waveguide 1 also becomes a propagating field. This multi-mode characteristic causes perturbations on 
values of the reflection coefficient versus the operating frequency, as evidenced in Fig. 3.10.

A modified version of the junction-2-problem was considered. In this case, we used waveguides with three, and with four layers in each region, as listed in Table 3.3. The corresponding reflection coefficient we obtained with our method is shown in Fig 3.10, again, we have an excellent agreement with reference solution. Independently of the number of layers, if they have a small media difference between each other, we expect this behaves as a homogeneous waveguide.

\begin{tabular}{cccccc}
\hline Region & $\rho_{0}(\mathrm{~mm})$ & $\rho_{1}(\mathrm{~mm})$ & $\rho_{2}(\mathrm{~mm})$ & $\epsilon_{r 1}$ & $\epsilon_{r 2}$ \\
\hline 1 & 1.84 & 2.00 & 5.00 & 1.1 & 1 \\
2 & 1.5 & 2.00 & 5.00 & 1.1 & 1 \\
\hline \multicolumn{6}{c}{$\sigma=10^{-6}(\mathrm{~S} / \mathrm{m})$} \\
\hline
\end{tabular}

Table 3.2: Dimensions and parameters of junction 2.

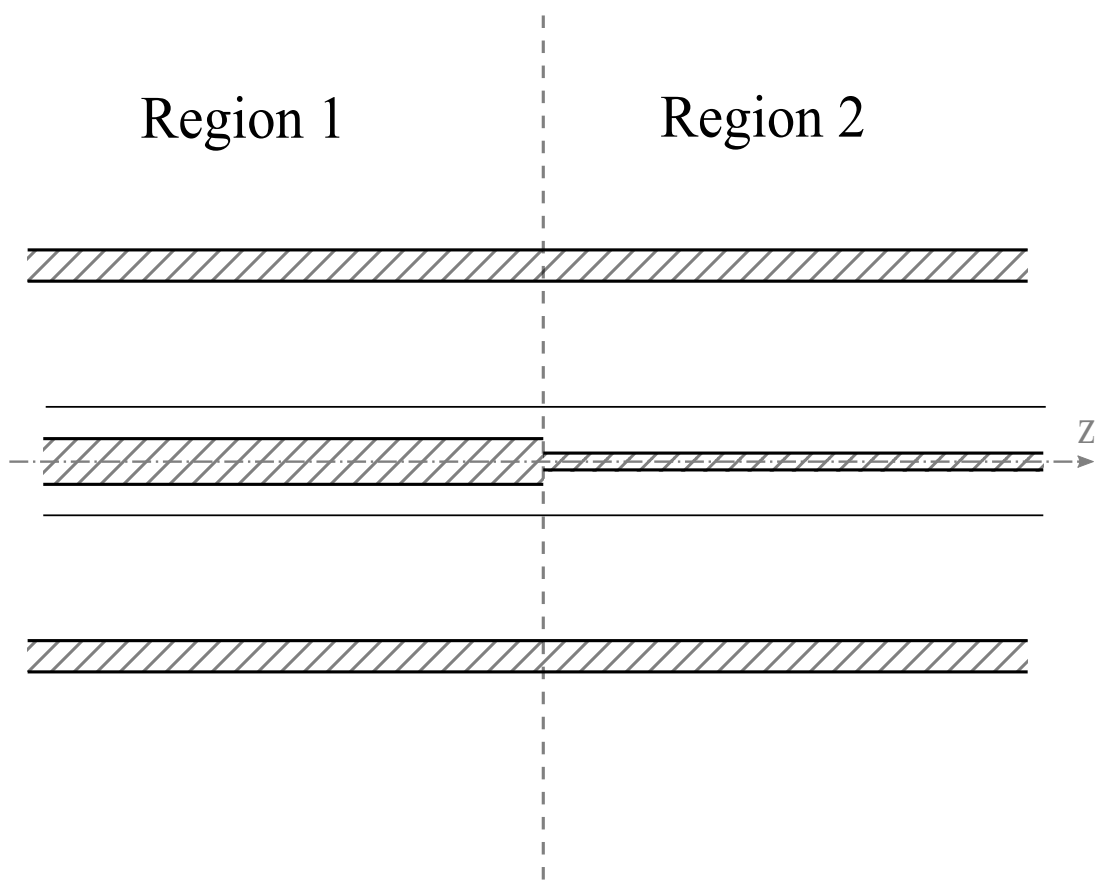

Figure 3.8: Geometry of junction 2.

\begin{tabular}{cccccccccc}
\hline Region & $\rho_{0}(\mathrm{~mm})$ & $\rho_{1}(\mathrm{~mm})$ & $\rho_{2}(\mathrm{~mm})$ & $\rho_{3}(\mathrm{~mm})$ & $\rho_{4}(\mathrm{~mm})$ & $\epsilon_{r 1}$ & $\epsilon_{r 2}$ & $\epsilon_{r 3}$ & $\epsilon_{r 4}$ \\
\hline 1 & 1.84 & 2.00 & 3.00 & 5.00 & - & 1.1 & 1 & 1 & - \\
2 & 1.5 & 2.00 & 3.00 & 4.00 & 5.00 & 1.1 & 1 & 1 & 1 \\
\hline \multicolumn{7}{c}{$\sigma=10^{-6}$} & $(\mathrm{~S} / \mathrm{m})$ \\
\hline
\end{tabular}

Table 3.3: Dimensions and parameters of modified junction 2. 


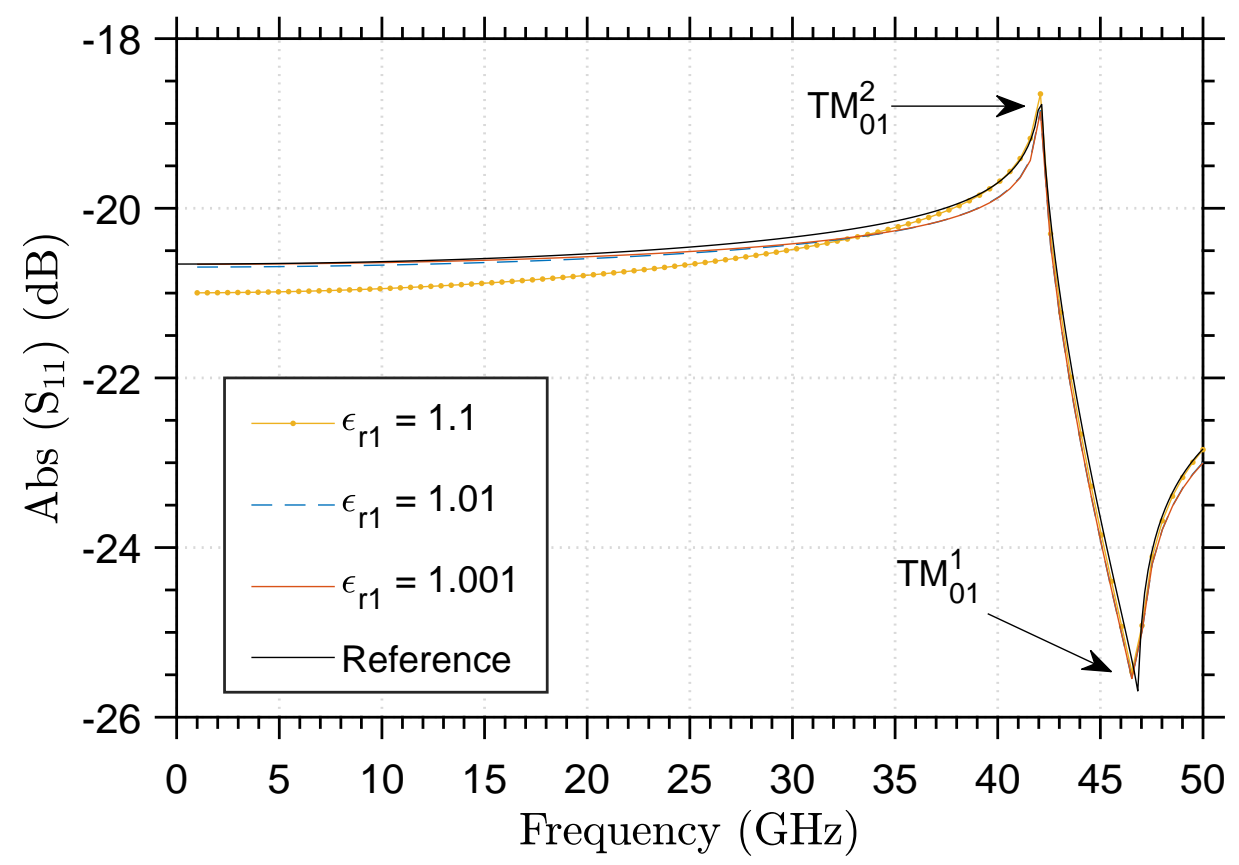

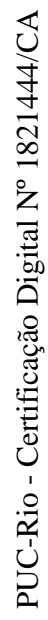

Figure 3.9: Reflection coefficient of junction 2 from 0.5 to $50 \mathrm{GHz}$ with two layers in each region.

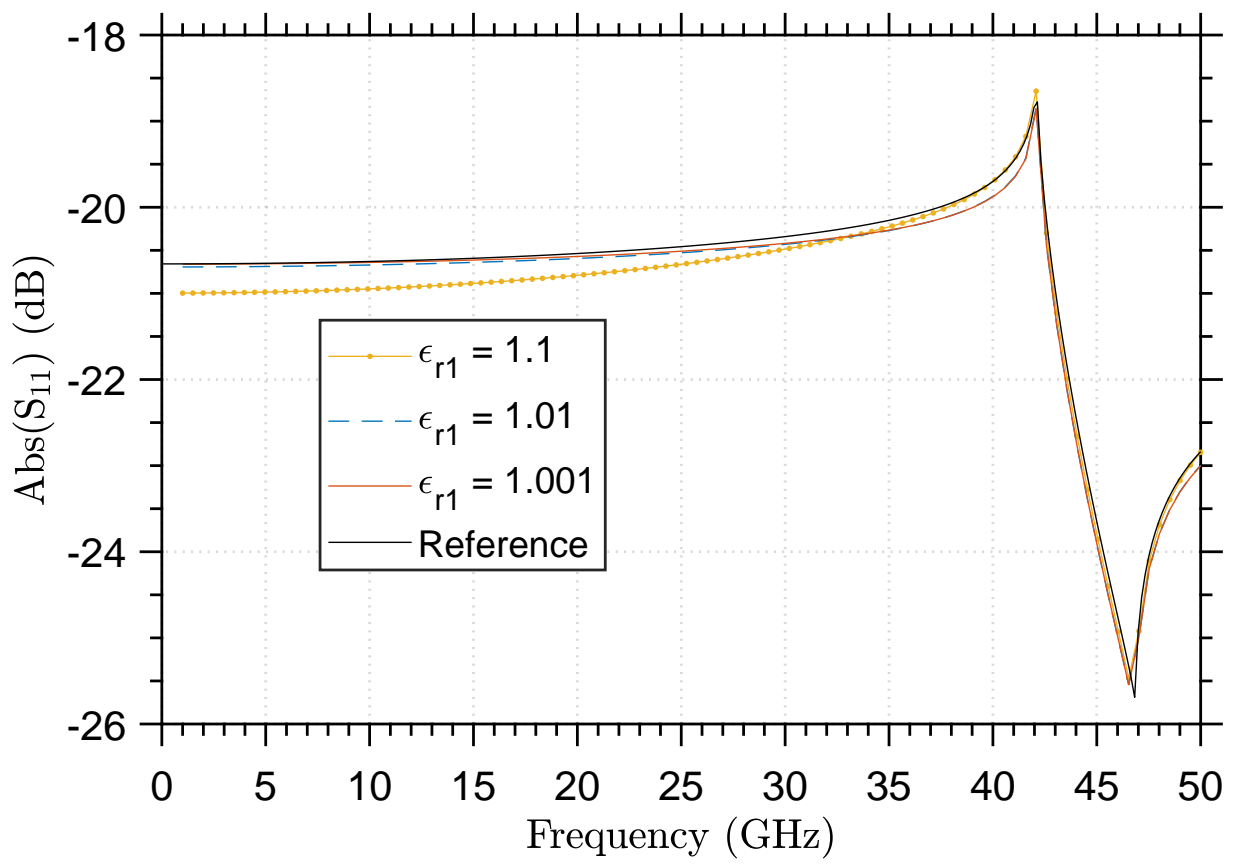

Figure 3.10: Reflection coefficient of modified junction 2 from 0.5 to $50 \mathrm{GHz}$ with three layers in region 1 and four layers in region 2.

\section{Junction 3}

To confirm that the statement made in previous section applies to every case scenario, a homogeneous to heterogeneous junction is now considered. The 
almost-homogeneous waveguide has three layers, with a slightly difference in the value of $\epsilon_{r}$ in the innermost layer.

Table 3.4 presents the parameters of junction 3 (see the geometry in Fig 3.11). The reflection coefficient as a function of the frequency for this case is presented in Fig 3.12. As $\epsilon_{r 1}$ become closer to 1, region 1 tends to a homogeneous waveguide and our results approximates to the reference one. Notice that the $\mathrm{TM}_{01}$ modes have cutoff frequency at $47 \mathrm{GHz}$ and $26.73 \mathrm{GHz}$ in regions 1 and 2, respectively. Near these frequencies, we observe a disturbance in the $S_{11}$ behavior caused by the propagation of such high-order modes.

We can also prove that our numerical algorithm works for regions with different number of layers, as region 1 has three layers while regions 2 has only two. Other examples about this topic will be given in the next sections.

\begin{tabular}{cccccccc}
\hline Region & $\rho_{0}(\mathrm{~mm})$ & $\rho_{1}(\mathrm{~mm})$ & $\rho_{2}(\mathrm{~mm})$ & $\rho_{3}(\mathrm{~mm})$ & $\epsilon_{r 1}$ & $\epsilon_{r 2}$ & $\epsilon_{r 3}$ \\
\hline 1 & 1.84 & 2.00 & 3.00 & 5.00 & 1.1 & 1 & 1 \\
2 & 1.5 & 4.84 & 5.00 & - & 2.55 & 1 & - \\
\hline \multicolumn{7}{c}{$\sigma=10^{-6}(\mathrm{~S} / \mathrm{m})$} \\
\hline
\end{tabular}

Table 3.4: Dimensions and parameters of junction 3.

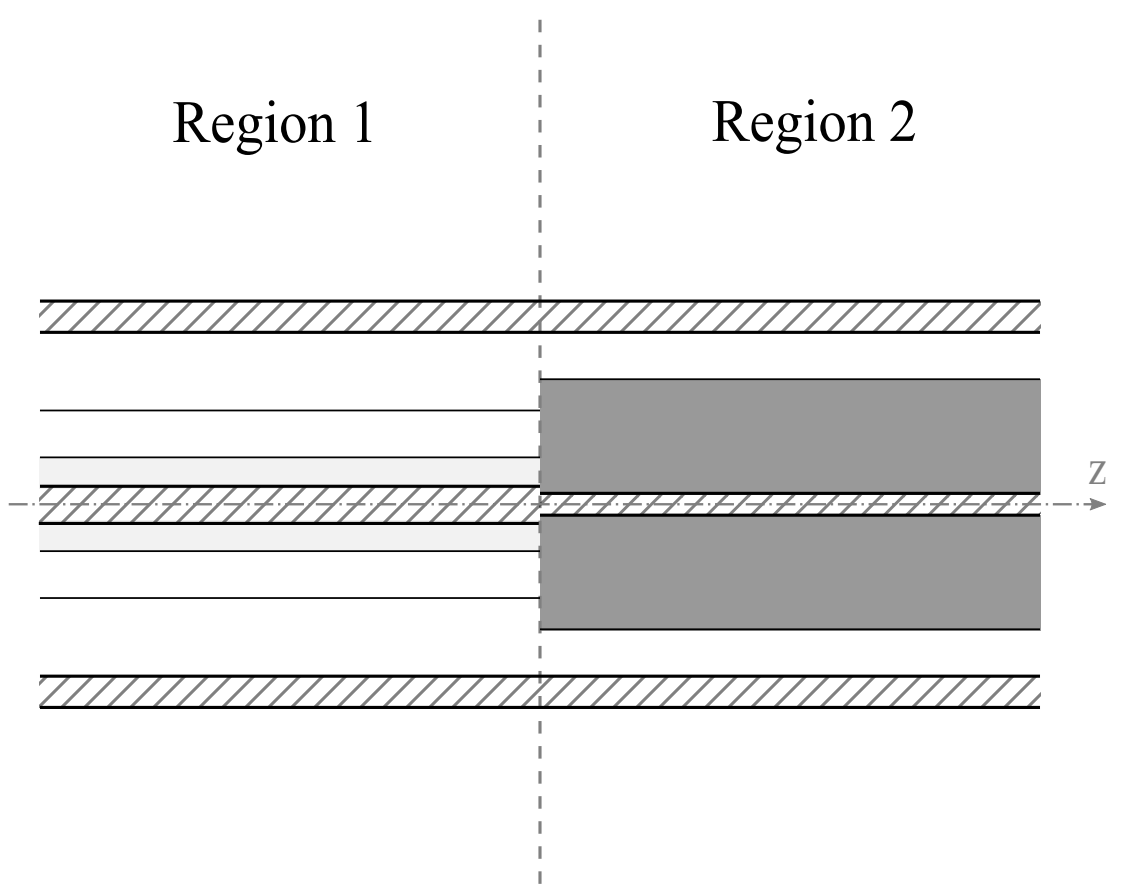

Figure 3.11: Geometry of junction 3. 


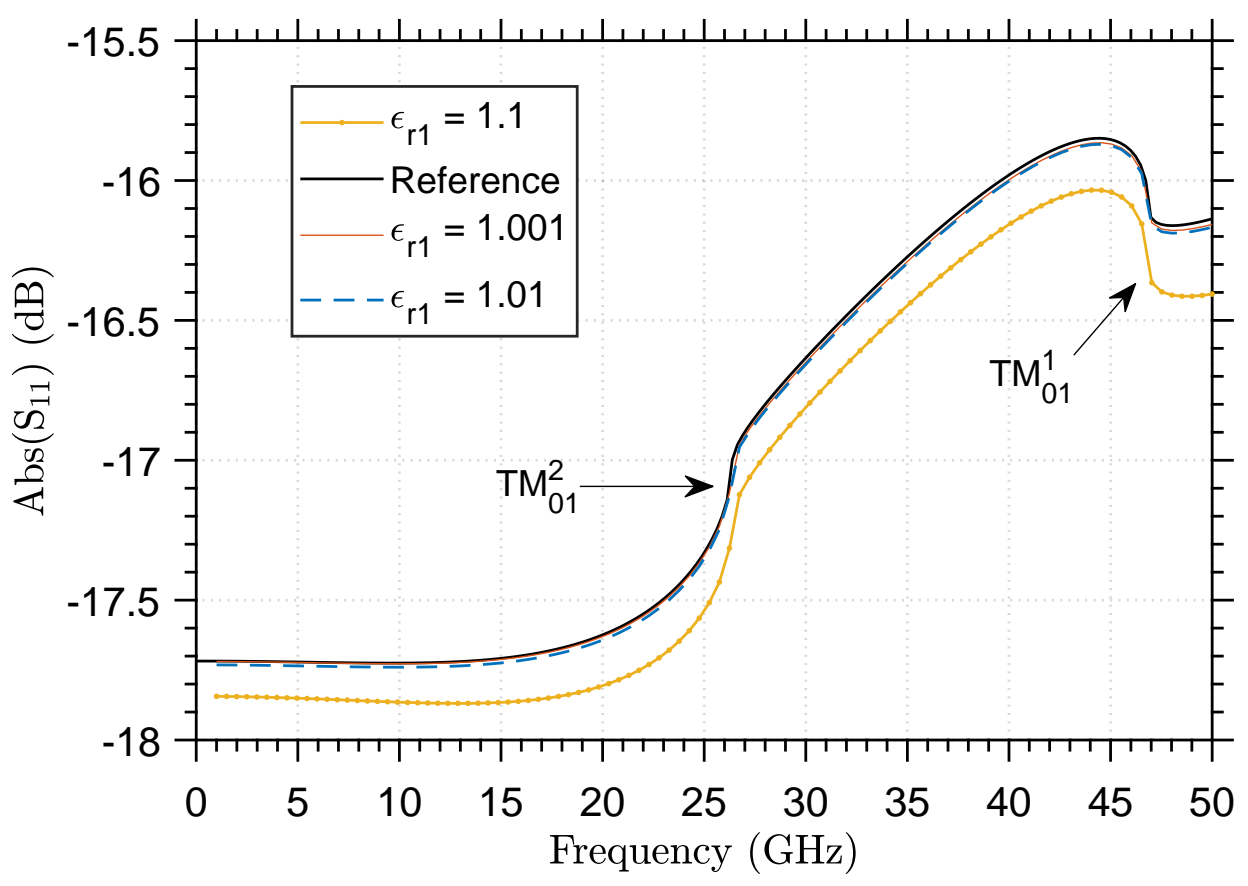

Figure 3.12: Reflection coefficient of junction 3 from 0.5 to $50 \mathrm{GHz}$.

\section{Junction 4}

Now, an inverse case is evaluated. A simulation of heterogeneous to homogeneous junction is proposed. Junction 4 consider the coupling of a 2 layer heterogeneous waveguide with a 4-layer guide, as depicted in Fig 3.13, and with the parameters listed in Table 3.5. Fig 3.14 presents results for different values of $\epsilon_{r 1}$. As the waveguide in region 2 has same parameters as waveguides from region 1 in the above-analyzed junctions 2 and 3, we can observe the same cutoff frequency at $47 \mathrm{GHz}$ for the mode $\mathrm{TM}_{01}$. The $\mathrm{TM}_{01}$ has its cutoff at $26.73 \mathrm{GHz}$ in region 1. As the operating frequency increases, such multi-mode coupling causes the disturbances observed in Fig. 3.14.

Also, the same observations made before can be repeated here: as the inner layer has its permittivity approaching to 1 , the reflection coefficient becomes near to the reference one. This confirms that our algorithm is able to modeling homogeneous waveguides.

\begin{tabular}{cccccccccc}
\hline Region & $\rho_{0}(\mathrm{~mm})$ & $\rho_{1}(\mathrm{~mm})$ & $\rho_{2}(\mathrm{~mm})$ & $\rho_{3}(\mathrm{~mm})$ & $\rho_{4}(\mathrm{~mm})$ & $\epsilon_{r 1}$ & $\epsilon_{r 2}$ & $\epsilon_{r 3}$ & $\epsilon_{r 4}$ \\
\hline 1 & 1.5 & 4.84 & 5.00 & - & - & 2.55 & 1 & - & - \\
2 & 1.84 & 2.00 & 3.00 & 4.00 & 5.00 & 1.1 & 1 & 1 & 1 \\
\hline \multicolumn{7}{c}{$\sigma=10^{-6}$} & $(\mathrm{~S} / \mathrm{m})$ \\
\hline
\end{tabular}

Table 3.5: Dimensions and parameters of junction 4 . 


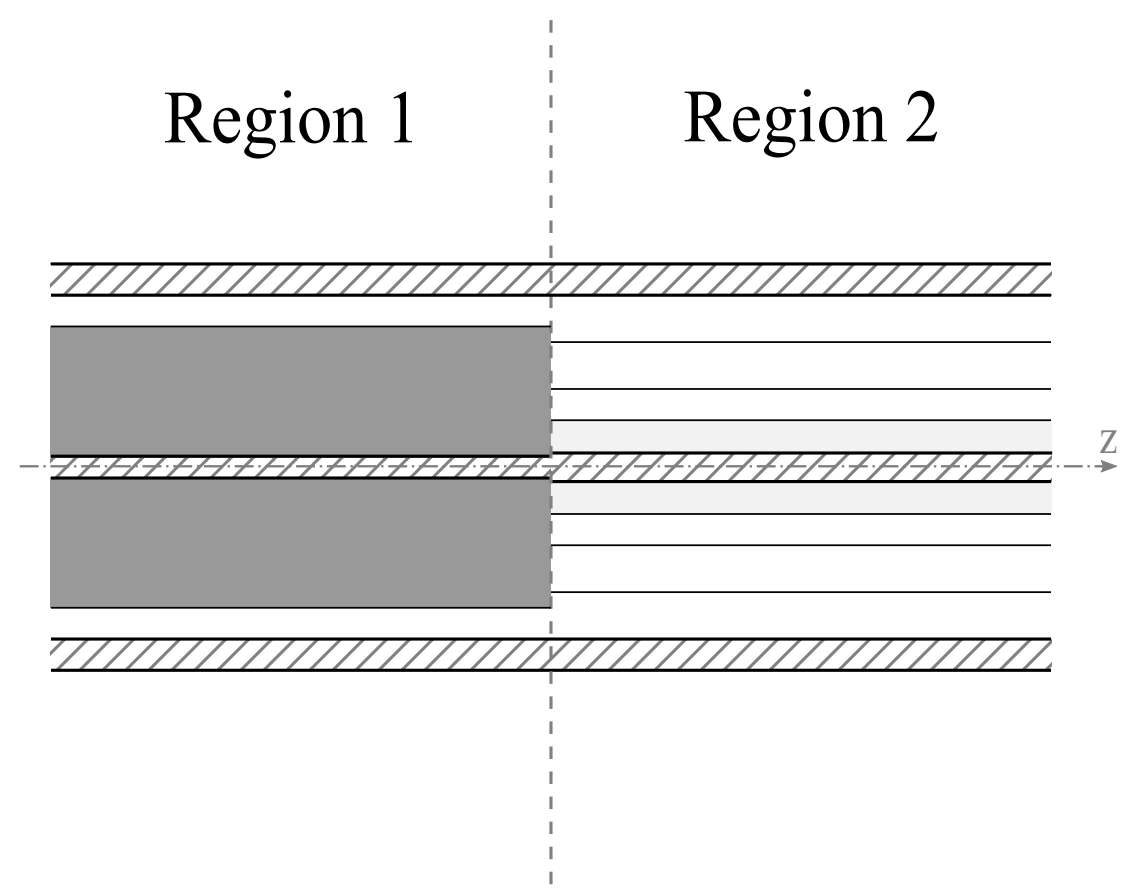

Figure 3.13: Geometry of junction 4.

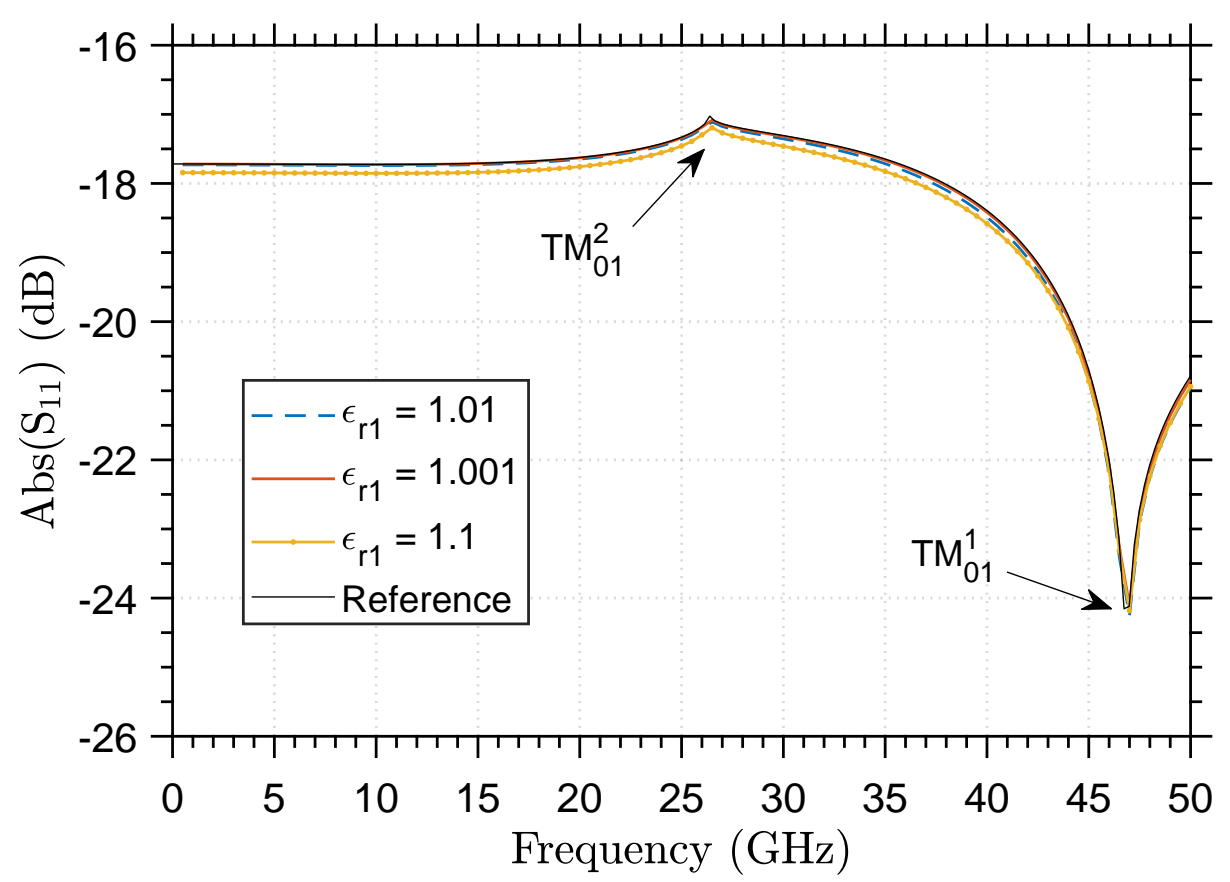

Figure 3.14: Reflection coefficient of junction 4 from 0.5 to $50 \mathrm{GHz}$.

\section{Junction 5}

The next junctions examined uses heterogeneous waveguides, and the FD results from CST were used as reference. We have considered 10 modes in each waveguide region for the MMT analysis of the next three junction problems. 
In Table 3.6 we shown the parameters of junction 5, which geometry is similar to that in Fig 3.6. The comparison between the $S_{11}$ results for the fundamental mode in region 1 obtained with our MMT-based method and with FD from CST are depicted as a function of the frequency in Fig 3.15. The curves are almost identical, with a small discrepancy noted for the higher frequencies (above $40 \mathrm{GHz}$ ). Such small differences does not disappear when the number of modes used in our solution was increased. We believe the FD discretization errors are undermining the accuracy of the solutions from CST.

For this junction, the $\mathrm{TM}_{01}$ modes starts to propagate at $38.62 \mathrm{GHz}$ and at $31.87 \mathrm{GHz}$ in region 1 and 2 , respectively, causing the variations with the frequency in the reflection coefficient curve observed in Fig 3.15.

The FD method from CST demanded 40 minutes and 14 seconds, using 5.807 GB of RAM memory, for simulating 50 frequency points of the reflection coefficient for mode 1 in region 1 . We have used a dedicated Workstation with a 2.10-GHz Intel Xeon E5-2620 v2 12-core processor. We have employed two symmetry planes for reducing the simulations domain size, and saving about 1/4 of RAM memory. This problem needed 110850 tetrahedrons for the discretization of the spacial domain. We used 4 and 3 modes in regions 1 and 2, respectively, for obtaining convergence. Only the excitation of the first modal field was considered. Notice that this not allowed us to obtain an entire GSM matrix, but only the modal reflection and transmission coefficients of the fundamental mode, from region 1 to 2. The counterpart coefficients from regions 2 to 1 can not be obtained unless additional simulations for each input and output excitation mode be performed.

In contrast, our method can compute the complete GSM matrix for 10 input (region 1) and 10 output (region 2) modes simultaneously. The $\mathrm{CPU}$ time required by our method was about 5 minutes and 40 seconds for computing 50 frequency points using a double-precision Matlab code running on a conventional laptop with an Intel core i7 processor. The memory required was less than $35 \mathrm{MB}$.

\begin{tabular}{cccccc}
\hline Region & $\rho_{0}(\mathrm{~mm})$ & $\rho_{1}(\mathrm{~mm})$ & $\rho_{2}(\mathrm{~mm})$ & $\epsilon_{r 1}$ & $\epsilon_{r 2}$ \\
\hline 1 & 1.84 & 3.00 & 5.00 & 2.55 & 1 \\
2 & 1.84 & 3.00 & 6.00 & 2.55 & 1 \\
\hline \multicolumn{5}{c}{$\sigma=10^{-6}(\mathrm{~S} / \mathrm{m})$} \\
\hline
\end{tabular}

Table 3.6: Dimensions and parameters of junction 5 . 


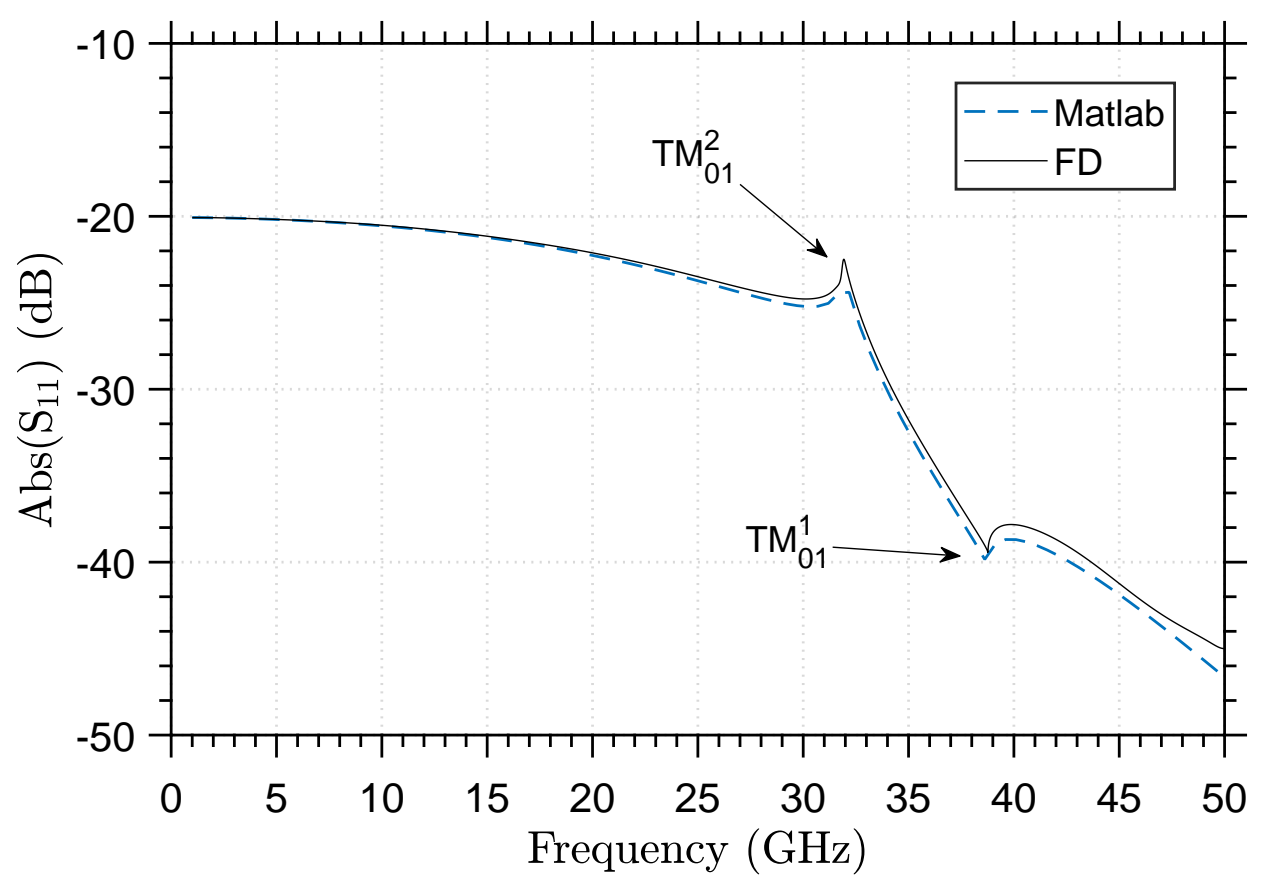

Figure 3.15: Reflection coefficient of junction 5 from 0.5 to $50 \mathrm{GHz}$.

\section{Junction 6}

We consider now the junction 6, whose parameters of the structure are detailed in Fig 3.16 and in Table 3.7. The reflection coefficient obtained via our approach and via FD are depicted in Fig 3.17. The curves are practically identical. The $\mathrm{TM}_{01}$ mode of region 2 becomes propagating at $32.18 \mathrm{GHz}$. At this frequency, the $\mathrm{TM}_{00}$ mode from regions 1 and 2 couples with the $\mathrm{TM}_{01}$ mode of region 2, causing the disturbance observed in Fig 3.17. At $36.14 \mathrm{GHz}$, the $\mathrm{TM}_{01}$ mode of region 1 becomes propagating and causes an additional disturbance.

\begin{tabular}{cccccccc}
\hline Region & $\rho_{0}(\mathrm{~mm})$ & $\rho_{1}(\mathrm{~mm})$ & $\rho_{2}(\mathrm{~mm})$ & $\rho_{3}(\mathrm{~mm})$ & $\epsilon_{r 1}$ & $\epsilon_{r 2}$ & $\epsilon_{r 3}$ \\
\hline 1 & 1.84 & 3.00 & 4.00 & 5.00 & 2.55 & 1 & 2.55 \\
2 & 1.84 & 3.00 & 6.00 & - & 2.55 & 1 & - \\
\hline \multicolumn{7}{c}{$\sigma=10^{-6}(\mathrm{~S} / \mathrm{m})$} \\
\hline
\end{tabular}

Table 3.7: Dimensions and parameters of junction 6 . 


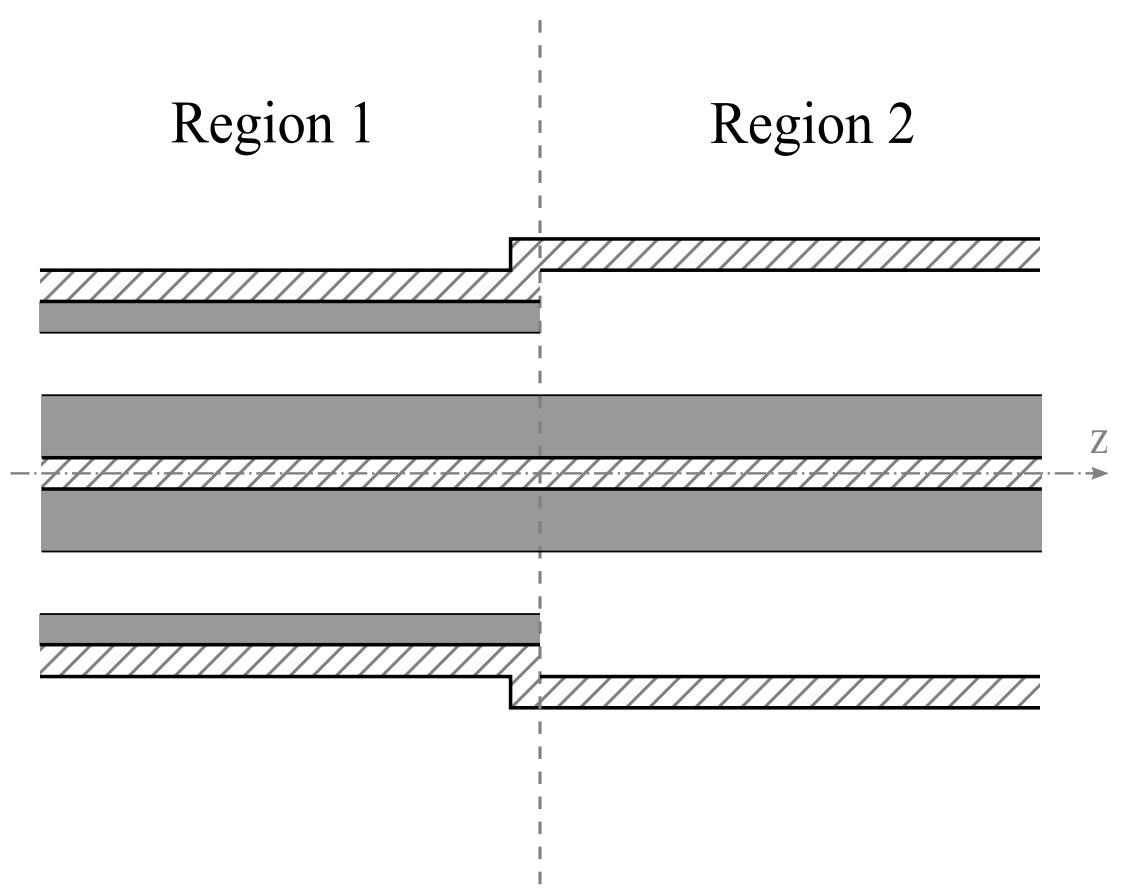

Figure 3.16: Geometry of junction 6 .

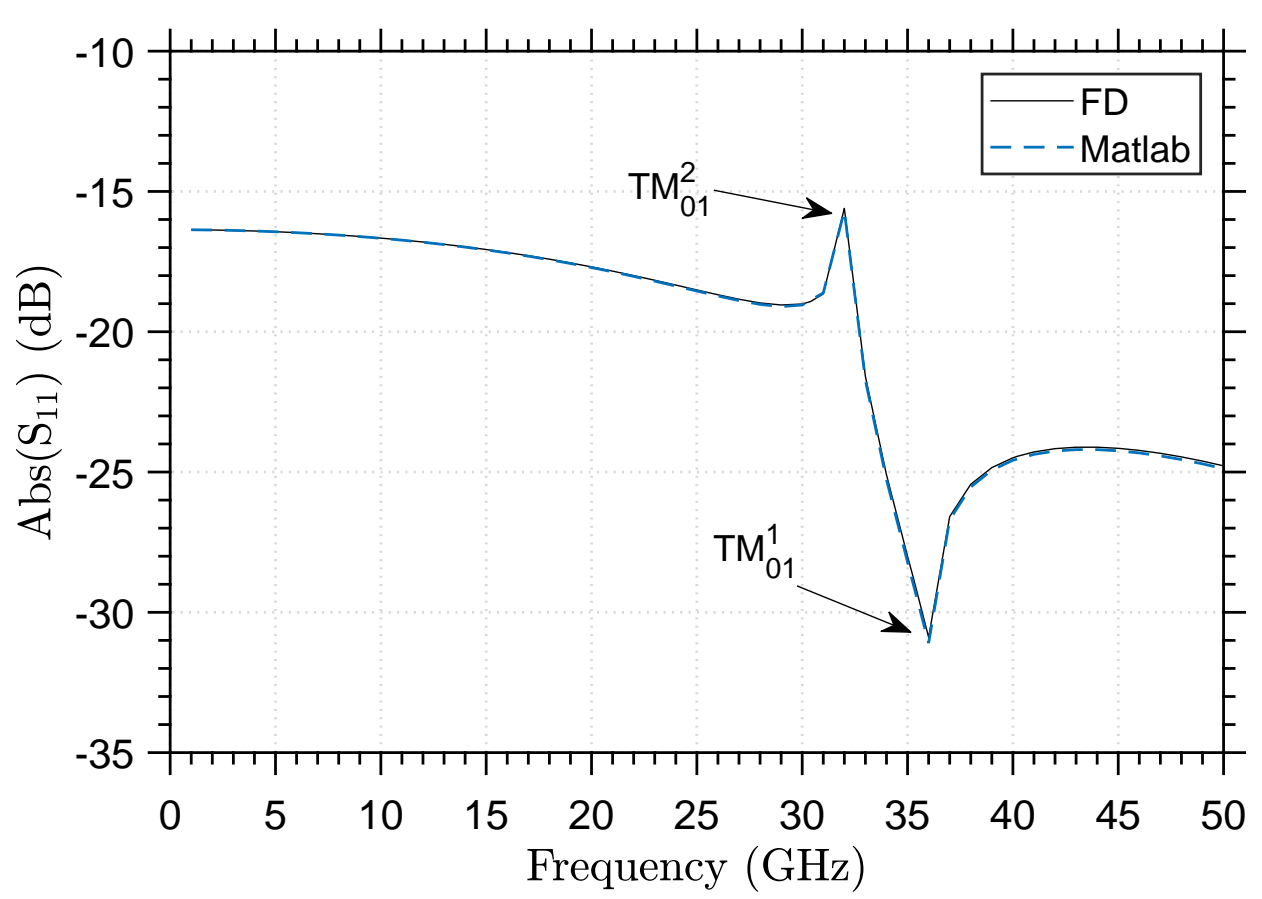

Figure 3.17: Reflection coefficient of junction 6 from 0.5 to $50 \mathrm{GHz}$.

\section{Junction 7}

Our junction 7 is depicted in Fig 3.18, and has the parameters described in Table 3.8. The results we have obtained via the implemented MMT-based algorithm are, again, in accordance with the FD results. A difference of 2.18 
$\mathrm{dB}$ can be seen at about $30.5 \mathrm{GHz}$, but this is due to the the small number of frequency points used in the FD simulation.

For this case, the cutoff frequency of $\mathrm{TM}_{01}$ in waveguide 1 is the same as previous junction, since both region 1 are identical. In waveguide 2 , however, $\mathrm{TM}_{01}$ mode changes from evanescent to propagating at $30.5 \mathrm{GHz}$. We can expect then, that for a more dense guide, the lower the cutoff frequency. Some simulations will be presented in the following to verify this.

\begin{tabular}{cccccccc}
\hline Region & $\rho_{0}(\mathrm{~mm})$ & $\rho_{1}(\mathrm{~mm})$ & $\rho_{2}(\mathrm{~mm})$ & $\rho_{3}(\mathrm{~mm})$ & $\epsilon_{r 1}$ & $\epsilon_{r 2}$ & $\epsilon_{r 3}$ \\
\hline 1 & 1.84 & 3.00 & 4.00 & 5.00 & 2.55 & 1 & 2.55 \\
2 & 1.84 & 3.00 & 5.00 & 6.00 & 2.55 & 1 & 2.55 \\
\hline \multicolumn{7}{c}{$\sigma=10^{-6}(\mathrm{~S} / \mathrm{m})$} \\
\hline
\end{tabular}

Table 3.8: Dimensions and parameters of junction 7.

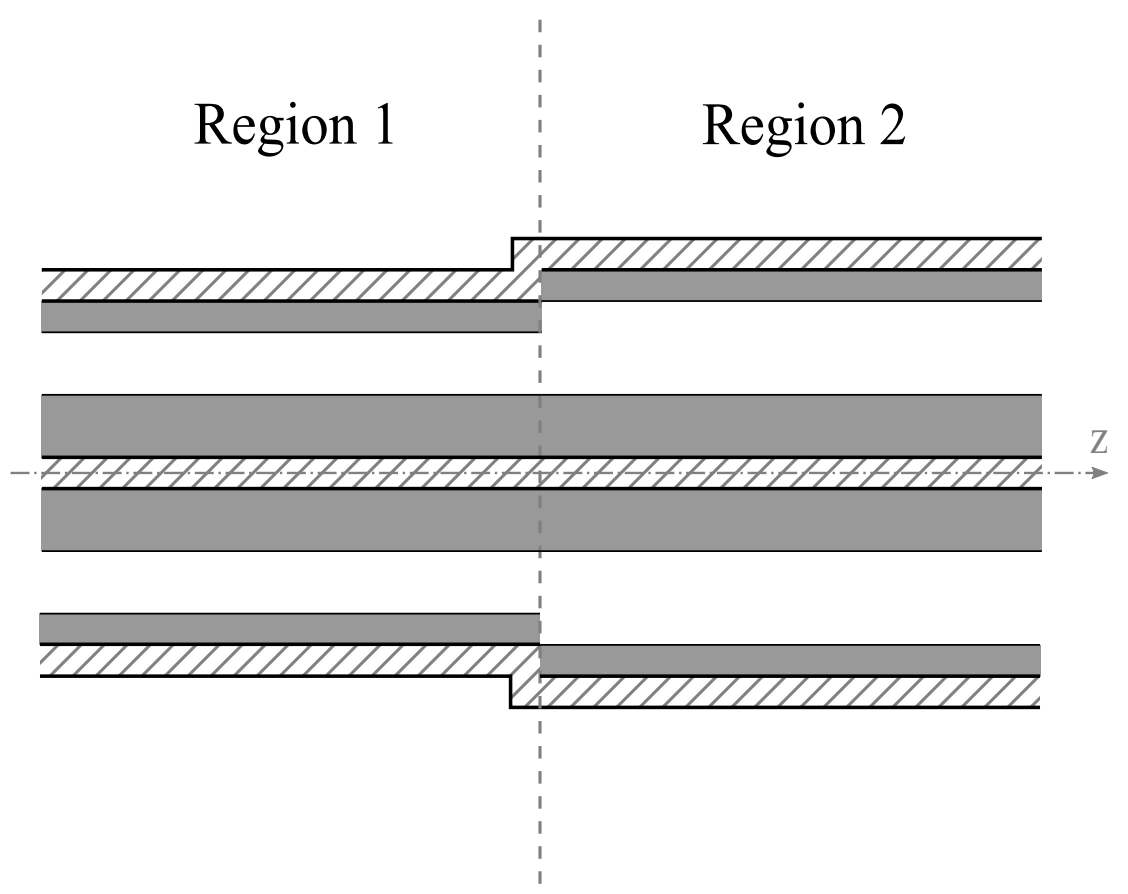

Figure 3.18: Geometry of junction 7. 


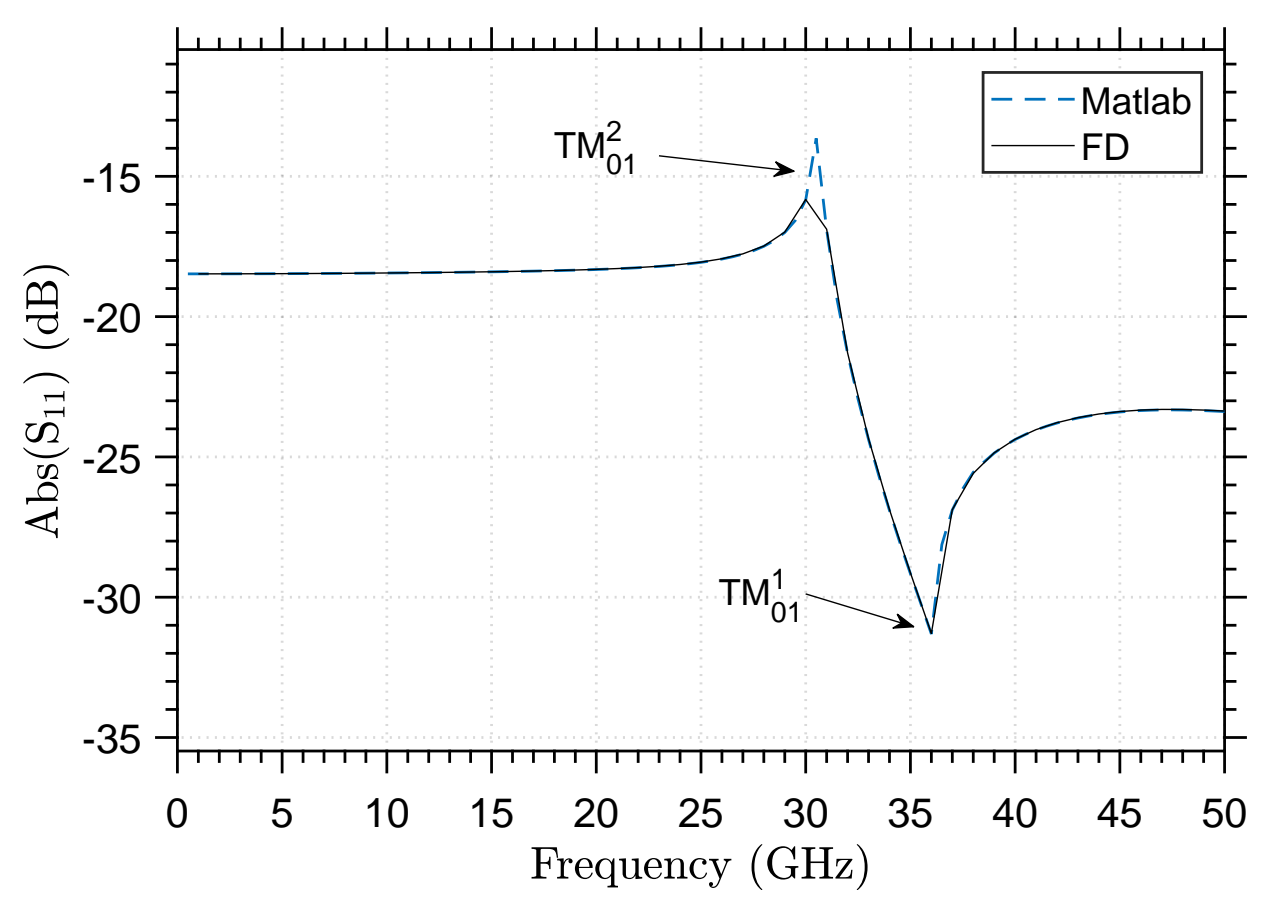

Figure 3.19: Reflection coefficient of junction 7 from 0.5 to $50 \mathrm{GHz}$.

\section{Junction 8}

The following structure (see the geometry in Fig 3.20) will be used in the next simulations to compare the reflection coefficient curves for materials with different values of permittivity. We will consider $\epsilon_{r}$ of distinct materials, obtained from [1], as shown in Table 3.10.

In all simulations, it was consider 10 modes. Comparison between the results found for each $\epsilon_{r}$ are in Fig 3.21. The curves found have similar shapes, but some observations can be made. As lower is the value of $\epsilon_{r}$, lower is the average value of the reflection coefficient. As the relative permittivity increases, more power will be reflected.

Another observation that can be done is that with higher $\epsilon_{r}$, we have a more dense material and the cutoff frequency will reduces. For region 1, these frequencies will be the same, independent of the permittivity. The $\mathrm{TM}_{01}$ mode starts propagating at $47 \mathrm{GHz}$, while the $\mathrm{TM}_{02}$ mode starts at $49.50 \mathrm{GHz}$. The cutoff frequencies in the waveguide 2 are presented in Table 3.11 for each of the materials investigated. As $\epsilon_{r}$ increases, more modes starts propagating inside the structure and they switch from evanescent to propagating early (in terms of the operating frequency). The peaks on the S-parameters observed in the curves are caused by the propagation of these modes, as indicated in figure Fig. 3.21. 
A junction of coaxial waveguides can be used as a measurement cell to determine the value of complex constitutive parameters via reflection or transmission methods [35-39]. Experimental values of $S_{11}$ and $S_{12}$ are obtained with the material under test inside the waveguide. An inversion process is then performed to obtain from these values the corresponding $\epsilon_{r}$ and $\mu_{r}$. Several different methods to calculate these complex parameters are discussed in details in [83], which also include the characterization of anisotropic materials. Results as the ones shown here can be used to evaluate the characteristics of the material under test, according to its $S_{11}$ curves.

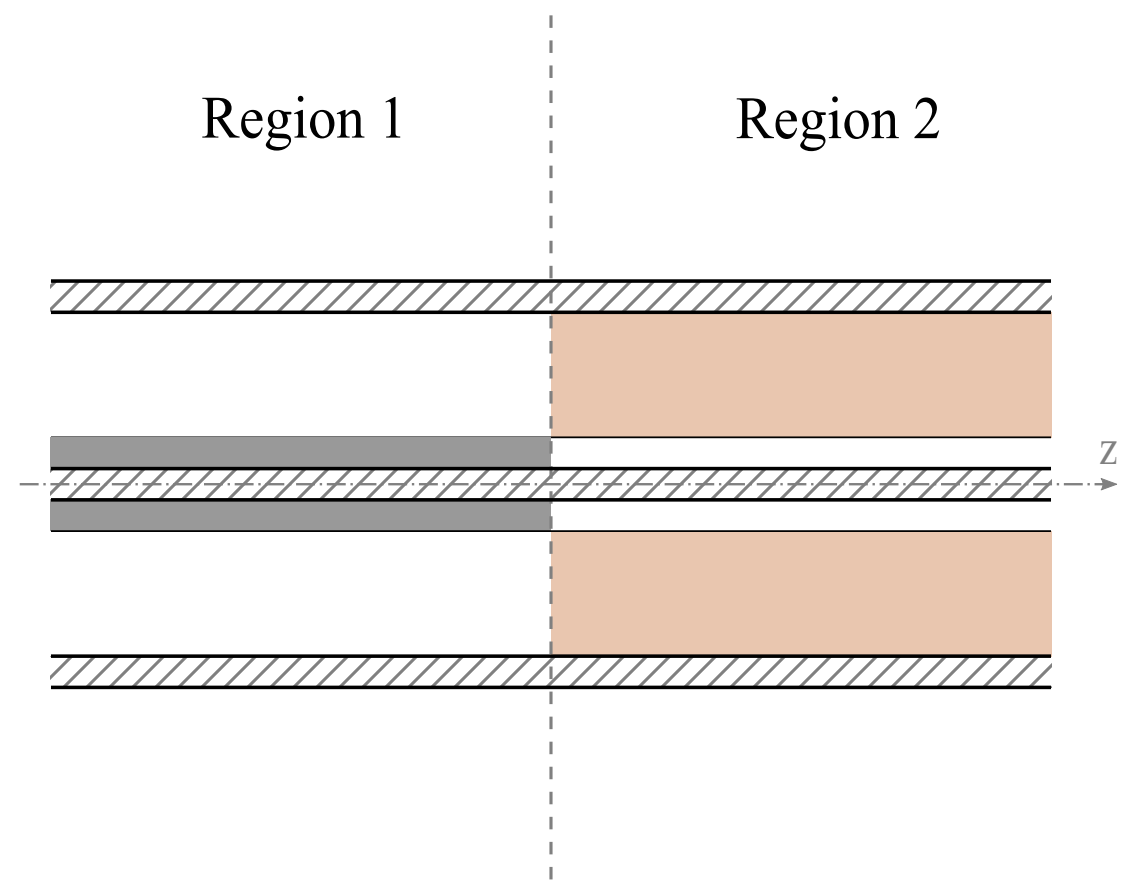

Figure 3.20: Geometry of junction 8. The filled area in region 2 represents the outermost radial layer with $\epsilon_{r 2}=3.8$.

\begin{tabular}{cccccc}
\hline Region & $\rho_{0}(\mathrm{~mm})$ & $\rho_{1}(\mathrm{~mm})$ & $\rho_{2}(\mathrm{~mm})$ & $\epsilon_{r 1}$ & $\epsilon_{r 2}$ \\
\hline 1 & 1.84 & 2.00 & 5.00 & 2.55 & 1 \\
2 & 1.84 & 2.00 & 5.00 & 1 & 3.8 \\
\hline \multicolumn{5}{c}{$\sigma=10^{-6}(\mathrm{~S} / \mathrm{m})$} \\
\hline
\end{tabular}

Table 3.9: Dimensions and parameters of junction 8 .

\begin{tabular}{cc}
\hline Material & $\epsilon_{r}$ \\
\hline Quartz & 3.8 \\
Mica & 6 \\
Marble & 8 \\
\hline
\end{tabular}

Table 3.10: Values of $\epsilon_{r}$ for different materials [1]. 


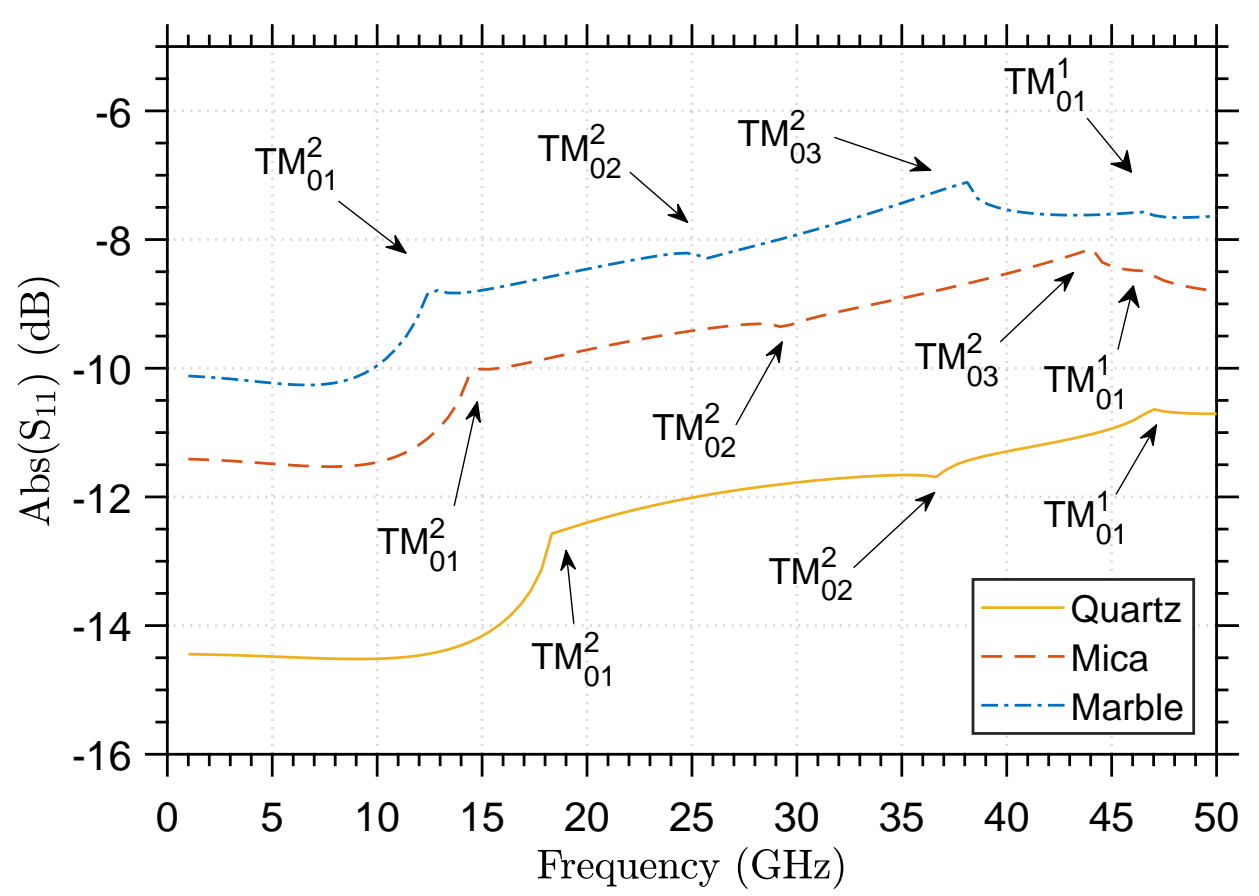

Figure 3.21: Reflection coefficient of junction 8, for different materials.

\begin{tabular}{cccc}
\hline Mode & $\epsilon_{r}=3.8$ & $\epsilon_{r}=6$ & $\epsilon_{r}=8$ \\
\hline $\mathrm{TM}_{01}$ & 18.32 & 14.85 & 12.38 \\
$\mathrm{TM}_{02}$ & 37.13 & 29.70 & 25.74 \\
$\mathrm{TM}_{03}$ & - & 44.5 & 38.61 \\
\hline
\end{tabular}

Table 3.11: Cutoff frequencies (in GHz) in region 2 for junction 8, with different values of $\epsilon_{r}$.

\section{4 .2}

\section{Coaxial to Circular Junctions}

\section{Junction 9}

A coaxial to circular junction with parameters given in Table 3.12 is analyzed in this section. This problem will be identified as junction 9. This structure comprises a homogeneous junction, as seen in Fig 3.22, considering the inner layer of both waveguides very similar to the outer one. For junction 2 , we noticed that only a two-digit difference was necessary to emulate a homogeneous waveguide. Then, the same criteria was considered in the next case studies. Results shown in Fig. 3.23 agree with those of reference from [33].

By examining the computed reflection coefficient, notice that below about $19 \mathrm{GHz}$, all the power is reflected. In region 1 we have a homogeneous coaxial waveguide, where the TEM mode propagates without cutoff. In region 2 , we 
have a circular guide, which does not supports a TEM mode. At about $19 \mathrm{GHz}$, the $\mathrm{TM}_{01}$ mode becomes propagating in region 2 and we have the coupling between TEM (from region 1) and $\mathrm{TM}_{01}$ (from region 2), allowing to a coupling of power along the junction. As observed in the figure, the peak in the $S_{11}$ is due to the presence of $\mathrm{TM}_{02}$ mode in region 2, whose cutoff is at $30.69 \mathrm{GHz}$.

\begin{tabular}{cccccc}
\hline Region & $\rho_{0}(\mathrm{~mm})$ & $\rho_{1}(\mathrm{~mm})$ & $\rho_{2}(\mathrm{~mm})$ & $\epsilon_{r 1}$ & $\epsilon_{r 2}$ \\
\hline 1 & 1.84 & 2.00 & 5.00 & 1.01 & 1 \\
2 & 0 & 2.00 & 6.00 & 1.01 & 1 \\
\hline \multicolumn{5}{c}{$\sigma=10^{-6}(\mathrm{~S} / \mathrm{m})$} \\
\hline
\end{tabular}

Table 3.12: Dimensions and parameters of junction 9 .

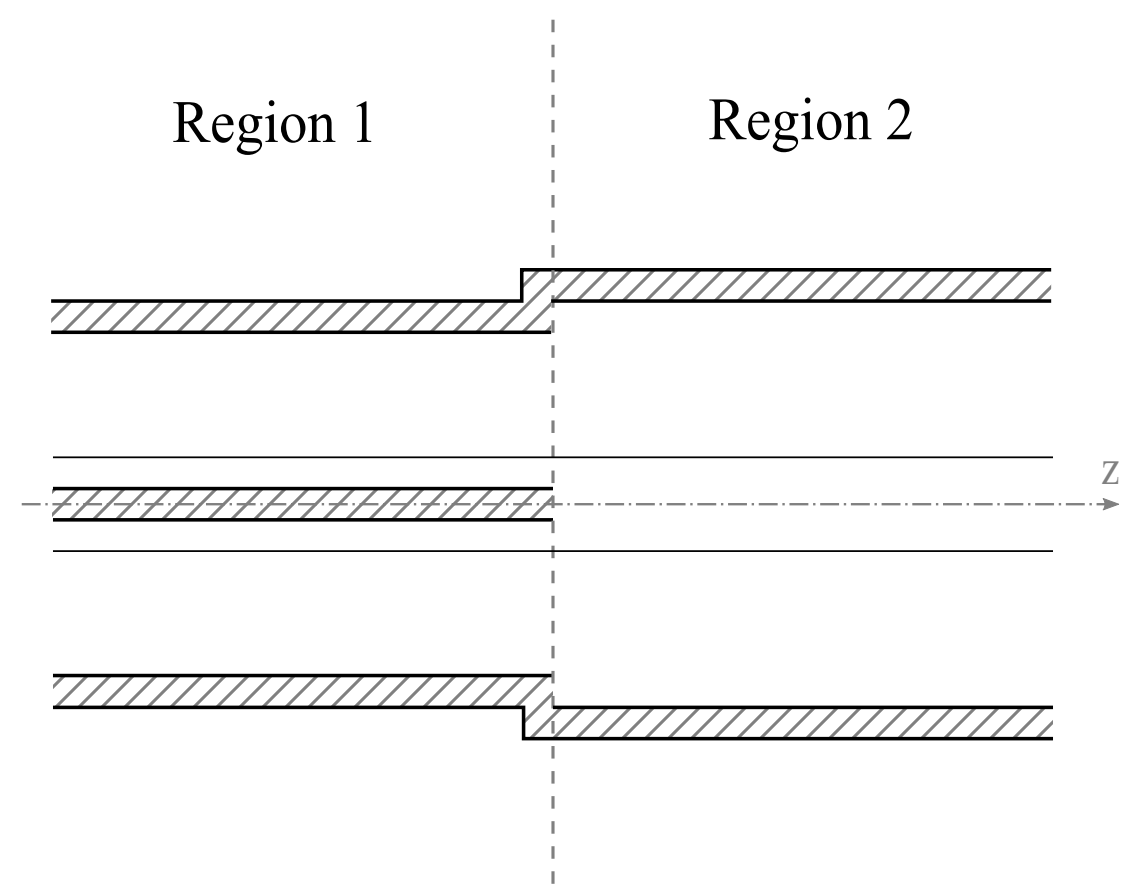

Figure 3.22: Geometry of junction 9 . 


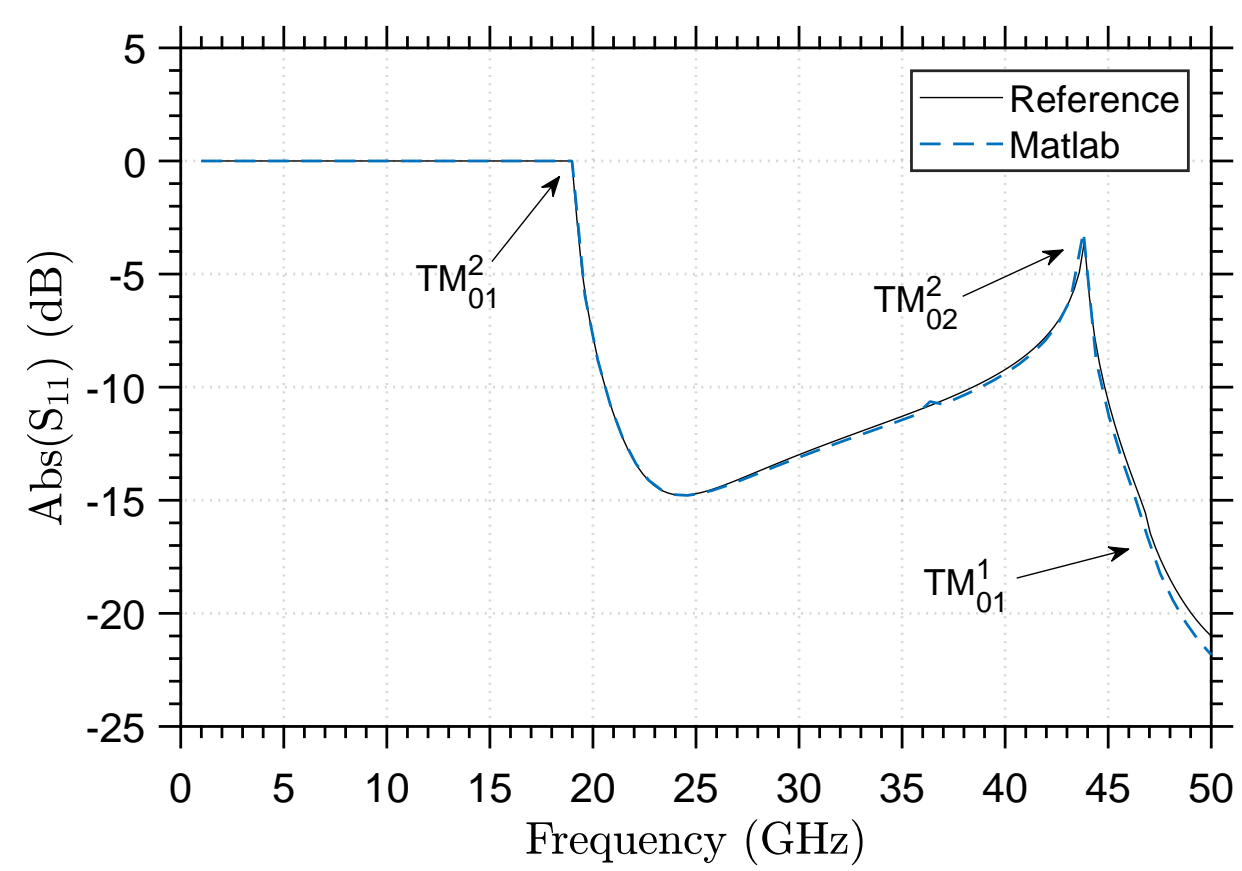

Figure 3.23: Reflection coefficient of junction 9 .

\section{Junction 10}

Junction 10 (detailed in Table 3.13 and in Fig 3.24) is a junction of a homogeneous coaxial waveguide with a heterogeneous circular output guide. Again, total reflection $\left(S_{11}=1\right)$ can be observed below the cutoff frequency of the first TM propagating mode in region 2. In Fig 3.25, we can see that the power coupling only starts over $15 \mathrm{GHz}$. Above the said frequency, the reflection coefficient reduces significantly. The disturbance observed in this case is caused by the $\mathrm{TM}_{03}$ mode, that becomes propagating in region 2 at $36.35 \mathrm{GHz}$.

\begin{tabular}{cccccc}
\hline Region & $\rho_{0}(\mathrm{~mm})$ & $\rho_{1}(\mathrm{~mm})$ & $\rho_{2}(\mathrm{~mm})$ & $\epsilon_{r 1}$ & $\epsilon_{r 2}$ \\
\hline 1 & 1.84 & 2.00 & 5.00 & 1.01 & 1 \\
2 & 0 & 2.00 & 6.00 & 2.55 & 1 \\
\hline \multicolumn{5}{c}{$\sigma=10^{-6}(\mathrm{~S} / \mathrm{m})$} \\
\hline
\end{tabular}

Table 3.13: Dimensions and parameters of junction 10. 


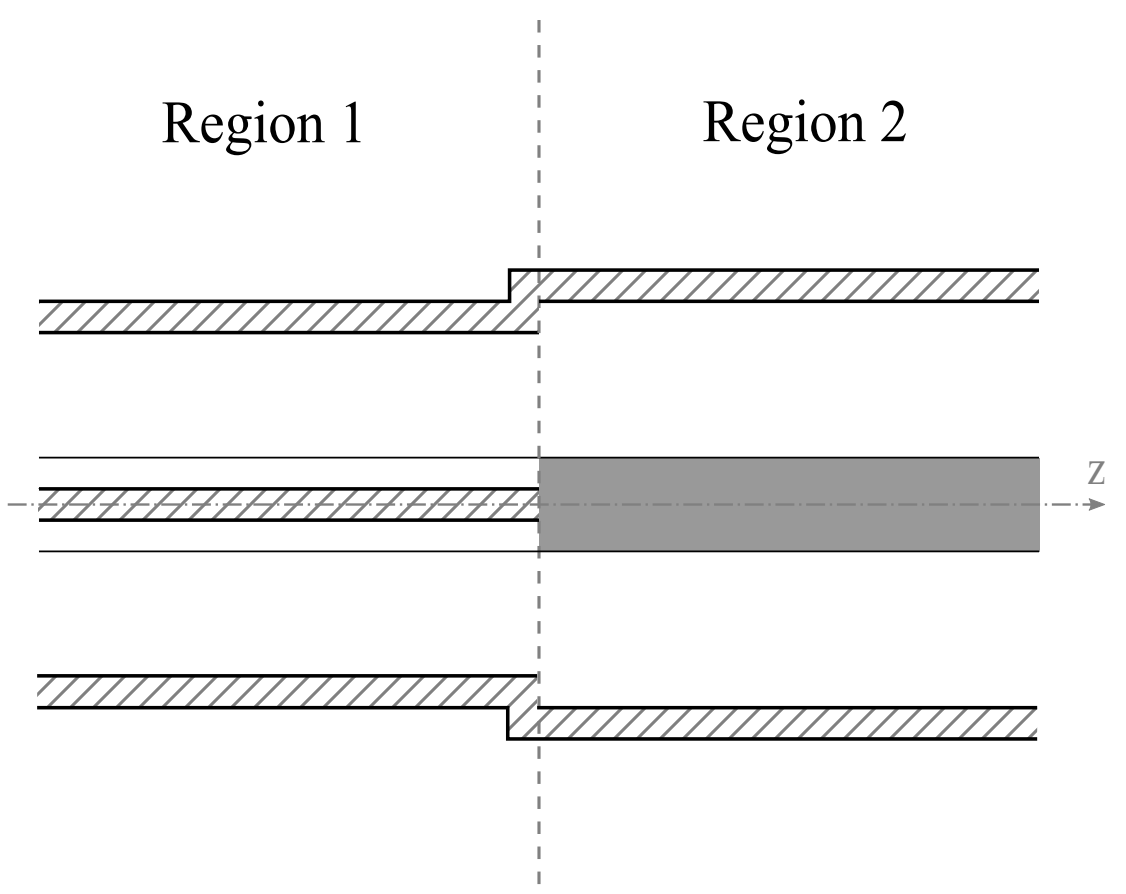

Figure 3.24: Geometry of junction 10.

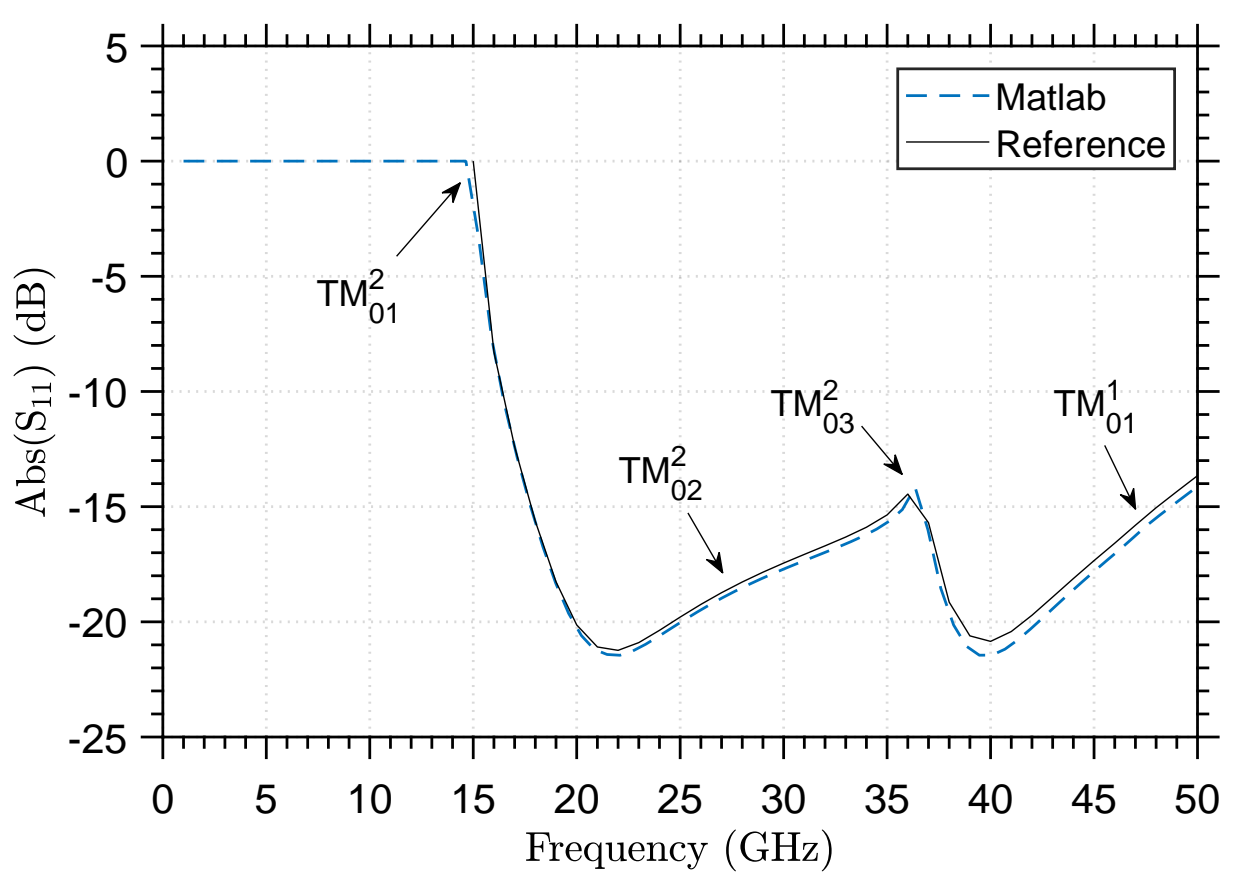

Figure 3.25: Reflection coefficient of junction 10. 


\subsection{3}

\section{Circular to Circular Junctions}

\section{Junction 11}

The next two structures are junctions of circular homogeneous waveguides. Junction 11 has the parameters listed in Table 3.14. The two waveguides have only one layer and are filled with air. The FD solver of CST was used again for comparison versus the presented approach. Good agreements between FD and our approach can be seen in Fig. 3.26.

As the two waveguides are circular, the $\mathrm{TM}_{00}$ or TEM modes are not supported, as in the previous cases. The first propagating field will be associated with the $\mathrm{TM}_{01}$ mode, whose cutoff frequency is $23.17 \mathrm{GHz}$ in region 1 , and $19.29 \mathrm{GHz}$ in region 2. Consequently, the power coupling from one region to the other starts close to $23 \mathrm{GHz}$, as seen in Fig 3.26.

\begin{tabular}{cccc}
\hline Region & $\rho_{0}(\mathrm{~mm})$ & $\rho_{1}(\mathrm{~mm})$ & $\epsilon_{r 1}$ \\
\hline 1 & 0 & 5.00 & 1 \\
2 & 0 & 6.00 & 1 \\
\hline \multicolumn{4}{c}{$\sigma=10^{-6}(\mathrm{~S} / \mathrm{m})$} \\
\hline
\end{tabular}

Table 3.14: Dimensions and parameters of junction 11.

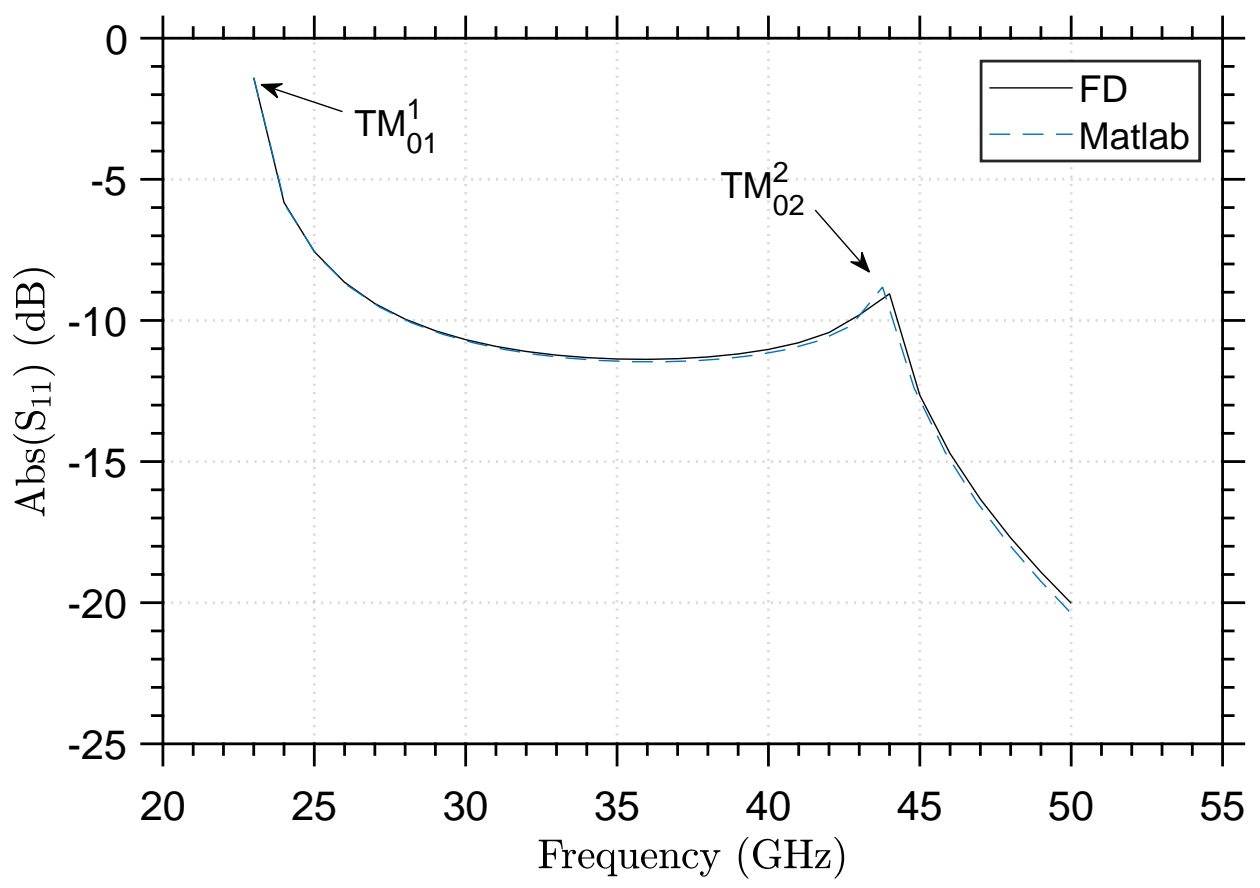

Figure 3.26: Reflection coefficient of junction 11. 


\section{Junction 12}

The second circular-to-circular junction we will investigate has parameters given in Table 3.15. It has the same structure of junction 11, but now, uniformly filled with a dielectric medium. In this scenario, the first propagating mode in regions 1 and 2 are $\mathrm{TM}_{01}$. The cutoff frequencies are at $14.68 \mathrm{GHz}$ and $12.18 \mathrm{GHz}$, respectively, in regions 1 and 2. For this reason, the power conversion is only possible for operating frequencies above $14.68 \mathrm{GHz}$. The reflection coefficient calculated by our method versus the FD results are presented in Fig. 3.27. The peaks observed in the figure are caused by the emergence of the high-order propagating modes. In region 1 , the $\mathrm{TM}_{02}$ mode becomes propagating at $33.1 \mathrm{GHz}$, and in region 2 , the $\mathrm{TM}_{02}$ and $\mathrm{TM}_{03}$ start at $27.73 \mathrm{GHz}$ and $43 \mathrm{GHz}$, respectively.

In comparison with junction 11, it is possible to observe that the cutoff frequencies from waveguide 1 and 2 have reduced in the dielectric-filled waveguide. As a consequence, it have allowed more modes to be propagating.

\begin{tabular}{cccc}
\hline Region & $\rho_{0}(\mathrm{~mm})$ & $\rho_{1}(\mathrm{~mm})$ & $\epsilon_{r 1}$ \\
\hline 1 & 0 & 5.00 & 2.55 \\
2 & 0 & 6.00 & 2.55 \\
\hline \multicolumn{4}{c}{$\sigma=10^{-6}(\mathrm{~S} / \mathrm{m})$}
\end{tabular}

Table 3.15: Dimensions and parameters of junction 12.

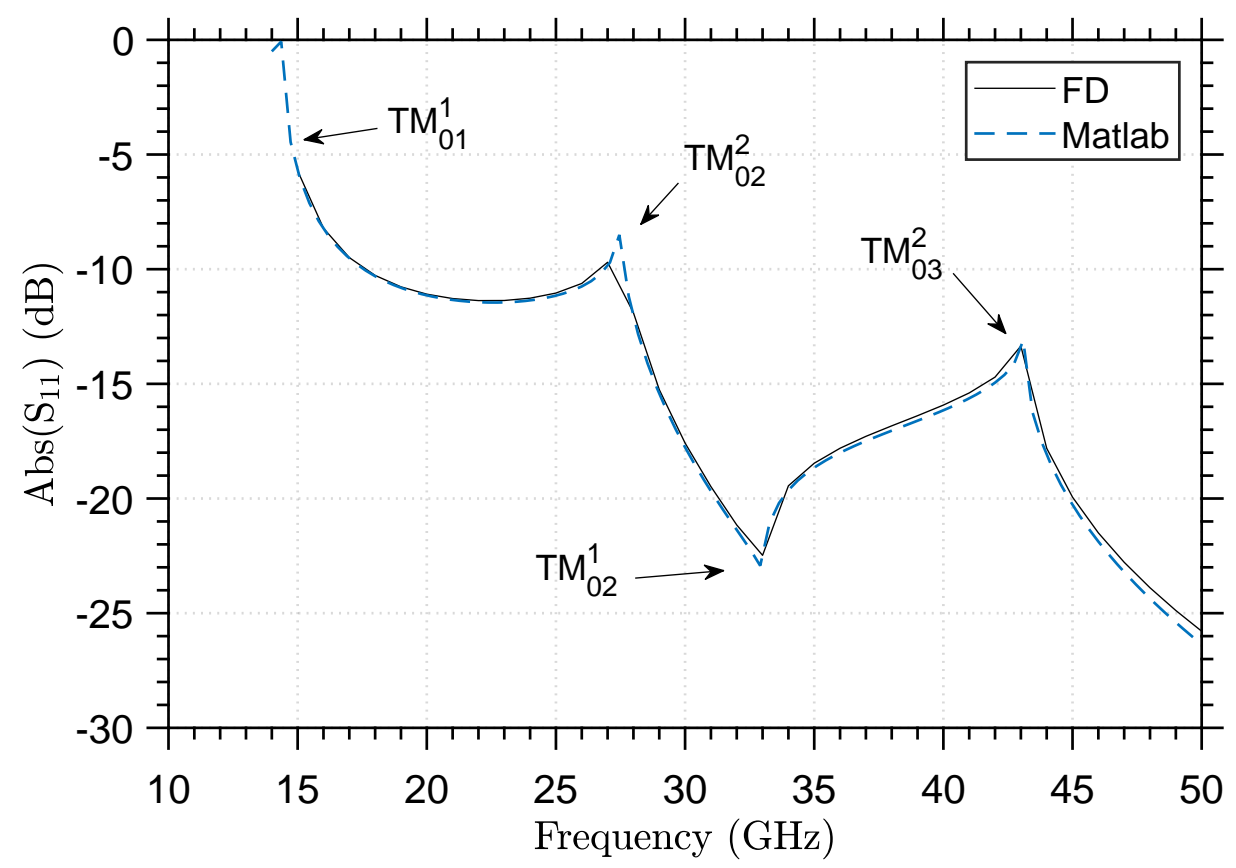

Figure 3.27: Reflection coefficient of junction 12. 


\section{4 .4}

\section{Structures with Multiple Junctions}

\section{Structure 1}

The following examples consider structures composed by three-waveguide junctions. The first structure is depicted in Fig 3.28, with the parameters given in Table 3.16. It was considered 10 modes in each region. This structure was analyzed before [33], and will be used here as a reference. Fig. 3.29 shows the results for the reflection coefficient of the first propagating mode in regions 1 obtained using our MMT-based solution as the frequency evolves. We can observe an excellent agreement of our solutions when compared to the MMT used in reference work [33].

This structure is a longitudinal-symmetric version of the junction 1. For this reason, region 1 (and consequently region 3, since they are equal) will share the same cutoff frequencies of the waveguide 1 used in junction 1 . The same applies to region 2 . The waveguides 1 and 3 of the present case study support TEM modes. In waveguide 2 , however, the non-homogeneous radial profile preclude the existence of a transmission-line TEM mode, but, instead, a $\mathrm{TM}_{00}$ mode will be supported. Notice that when the homogeneity vanishes the $\mathrm{TM}_{00}$ mode degenerates to a TEM one. Further details can be obtained in [33].

Although we have equal cutoffs, this will not cause the same disturbances in the reflection coefficient. The appearance of these modes will not lead to the same peaks observed in previous cases. As can be seen in the result obtained here, there are more oscillations than before and not necessarily they occur at cutoff frequencies. This can be explained due to internal reflections of the power in region 2 , that acts as a cavity of length $L_{2}$.

\begin{tabular}{ccccccc}
\hline Region & $\rho_{0}(\mathrm{~mm})$ & $\rho_{1}(\mathrm{~mm})$ & $\rho_{2}(\mathrm{~mm})$ & $\epsilon_{r 1}$ & $\epsilon_{r 2}$ & $\mathrm{~L}(\mathrm{~mm})$ \\
\hline 1 & 1.84 & 2.00 & 5.00 & 1.01 & 1 & $\infty$ \\
2 & 1.84 & 2.00 & 6.00 & 2.55 & 1 & 10 \\
3 & 1.84 & 2.00 & 5.00 & 1.01 & 1 & $\infty$ \\
\hline \multicolumn{7}{c}{$\sigma=10^{-6}(\mathrm{~S} / \mathrm{m})$} \\
\hline
\end{tabular}

Table 3.16: Dimensions and parameters of structure 1 . 


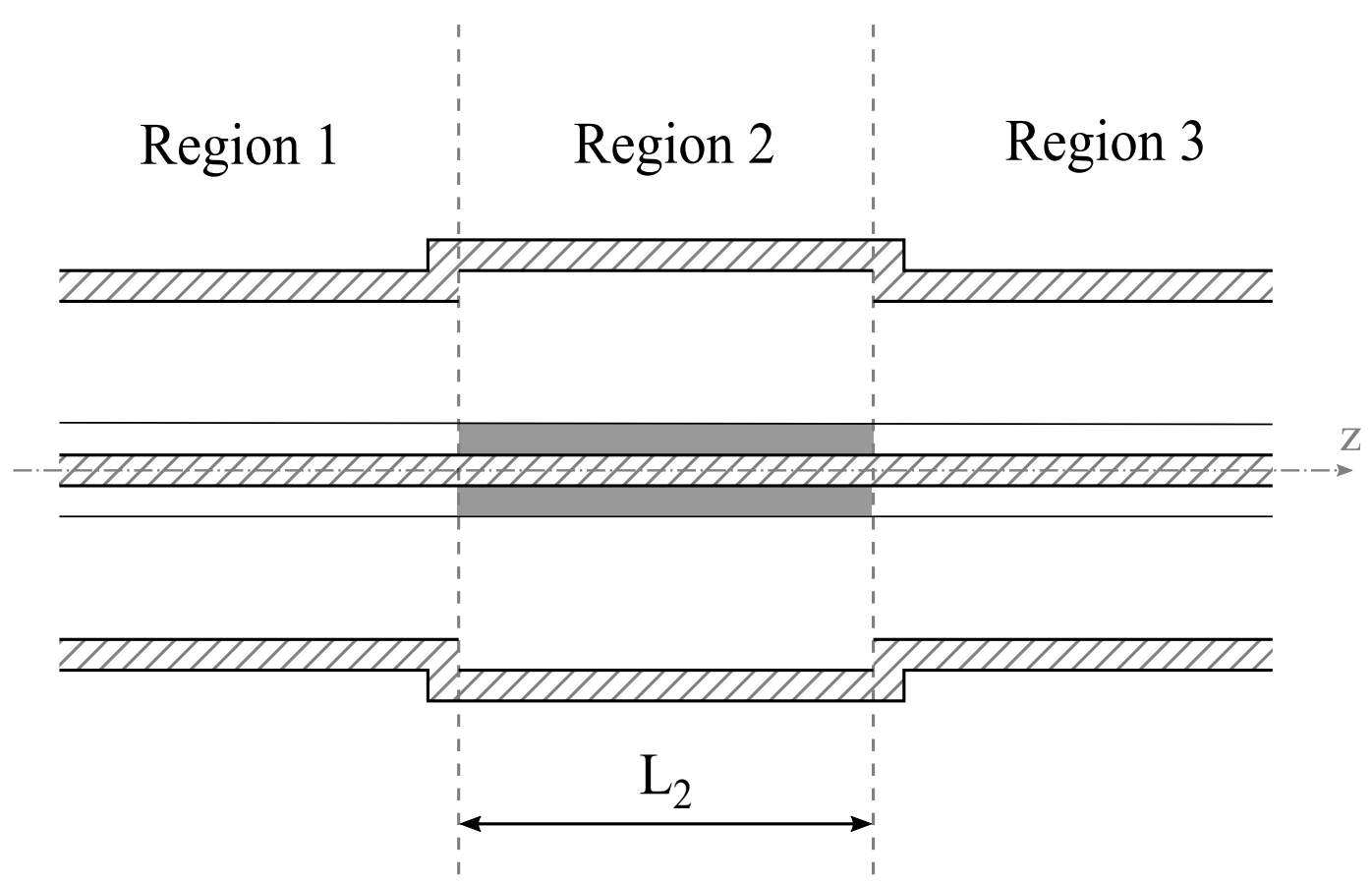

Figure 3.28: Geometry of structure 1.

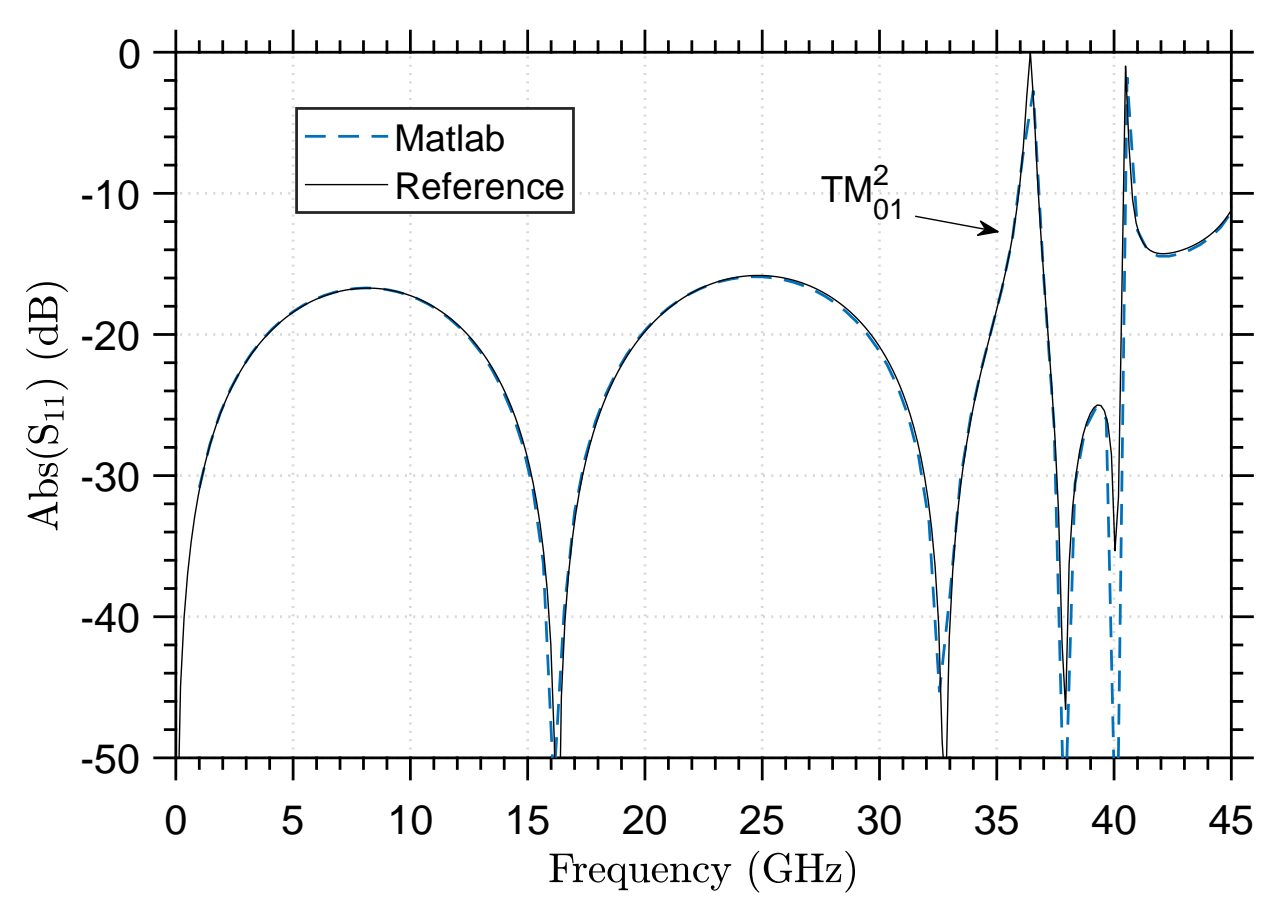

Figure 3.29: Reflection coefficient of structure 1.

\section{Structure 2}

Now, we consider a structure comprising the junction of three coaxial waveguides. The middle waveguide has three radial layers, while the other ones presents only two layers, as depicted in Fig. 3.30. The parameters of 
this structure are listed in Table 3.17. Fig. 3.31 shows a comparison of result obtained via our approach versus the FD ones. We have employed 20 modes for each waveguide region in our simulation. Despite some small differences, results are in good agreement.

The cutoff frequencies and the corresponding propagating TM modes are as follows: $14.85 \mathrm{GHz}$ for $\mathrm{TM}_{01}$ and $32.18 \mathrm{GHz}$ for $\mathrm{TM}_{02}$ in region 2 and 47.03 $\mathrm{GHz}$ for $\mathrm{TM}_{01}$ in region 1. Again, the appearance of these modes does not necessarily imply in a peak in the reflection coefficient, the oscillations seen are the result of multiple internal reflections caused in the waveguide of region 2 .

The structure 2 can be used as a measurement cell for complex permittivity and permeability characterization. For that, the sample should first be shaped as a cylinder with an inner role, forming a coaxial ring. In [84] it is explained that in coaxial cell measurements, the samples must be machined precisely to fit inside the structure and avoid air gaps. These gaps between the waveguide conductor and the sample may cause measurement errors and uncertainties. In [85], this problem is analyzed and some numerical approximations are given to consider it. The gap between the sample and the inner conductor of a coaxial guide can lead to large measurement error. Authors in [85] commented that such errors can be minimized by installing the around the outer conductor. One configuration that avoids this problem is the coaxial-circular-coaxial junction (that kind of structure will be analyzed in the following) since the segment of circular waveguide filled with the sample will not have any gaps in the middle of the cell. Since our formulation allows us to use a different number of layers in each region, these problem associated to imprecise samples can be overcomed by including these air gaps as very small layers in the coaxial cell. This will allow us to guarantee the accuracy of the results. In short, we are proposing that the sample under analyzes does not completely fill the measurement cell cross-section, intentionally. Standard modeling methods, however, does not apply for that scenarios and formalism as we presented in this work is paramount.

In order to verify the reflection coefficient for anisotropic materials,

\begin{tabular}{ccccccccc}
\hline Region & $\rho_{0}(\mathrm{~mm})$ & $\rho_{1}(\mathrm{~mm})$ & $\rho_{2}(\mathrm{~mm})$ & $\rho_{3}(\mathrm{~mm})$ & $\epsilon_{r 1}$ & $\epsilon_{r 2}$ & $\epsilon_{r 3}$ & $\mathrm{~L}(\mathrm{~mm})$ \\
\hline 1 & 1.84 & 2.00 & 5.00 & - & 2.55 & 1 & - & $\infty$ \\
2 & 1.50 & 3.00 & 5.00 & 7.00 & 2.55 & 3.8 & 2.55 & 10 \\
3 & 1.84 & 2.00 & 5.00 & - & 2.55 & 1 & - & $\infty$ \\
\hline \multicolumn{7}{c}{$\sigma=10^{-6}(\mathrm{~S} / \mathrm{m})$} \\
\hline
\end{tabular}

Table 3.17: Dimensions and parameters of structure 2. 


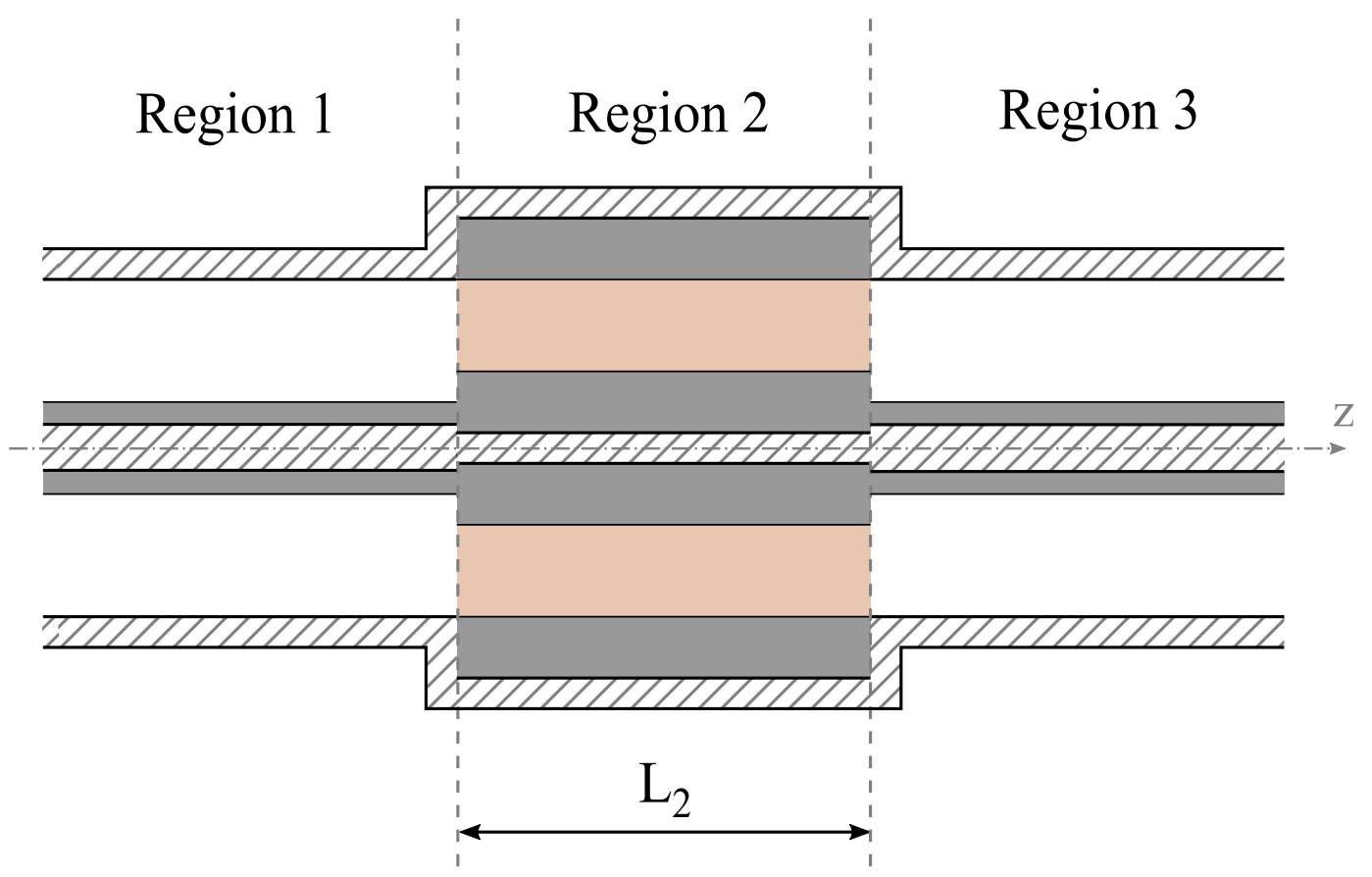

Figure 3.30: Geometry of structure 2.

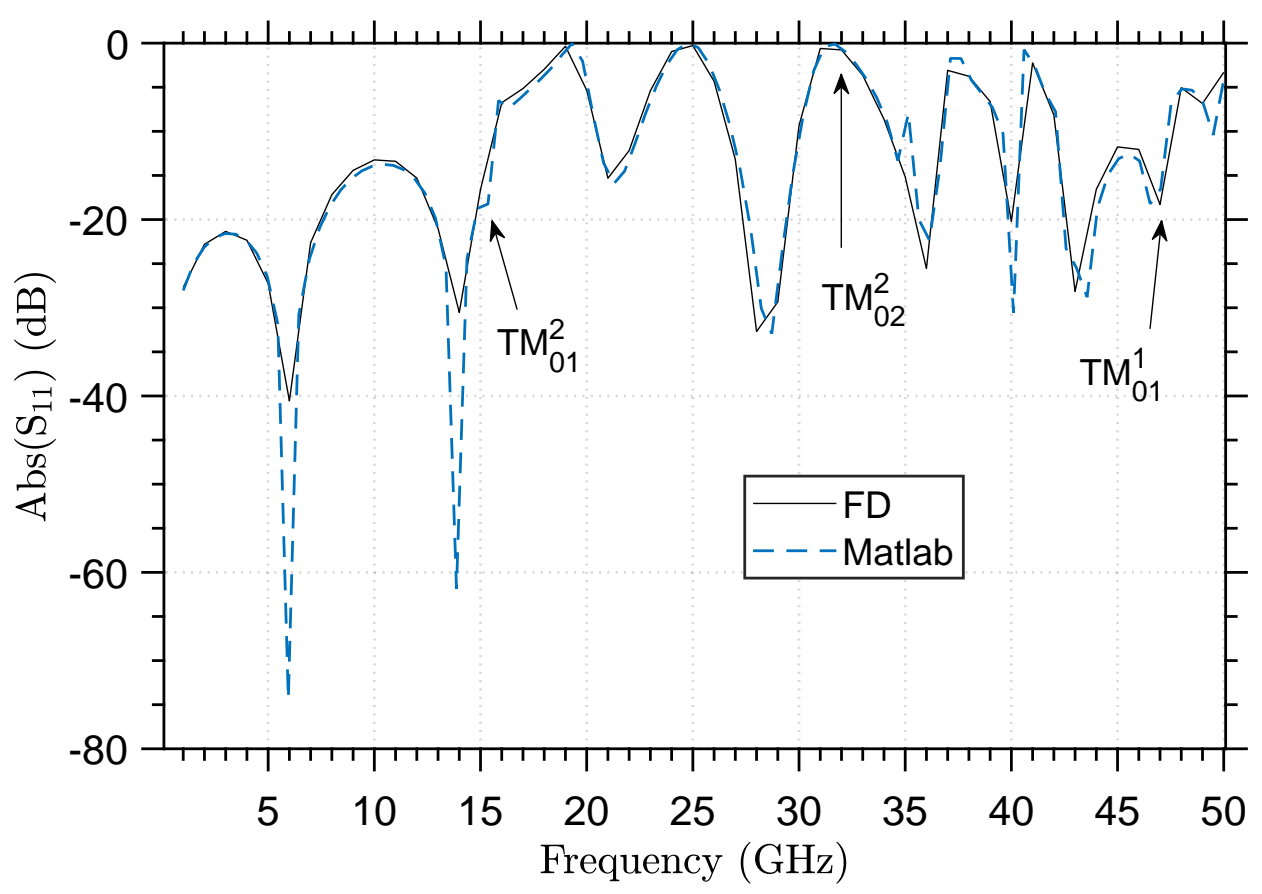

Figure 3.31: Reflection coefficient of structure 2.

structure 2 will be evaluated considering variations in the $z$ component of the permittivity tensor. The sample present in layer 2 of region 2 (colored layer in Fig 3.30) will have $\epsilon_{r s}=3.8$ and $\epsilon_{r z}=\{3.8,4.8,5.8\}$. A comparison of the results obtained can be observed in Fig 3.32. Until the $10 \mathrm{GHz}$ frequency, there are no variations, but as the frequency increases, a discrepancy on the 
curves is noticed. Although the values of the $S_{11}(d B)$ are different, we can not confirm any type of pattern as we increase the anisotropy value with this simulation.

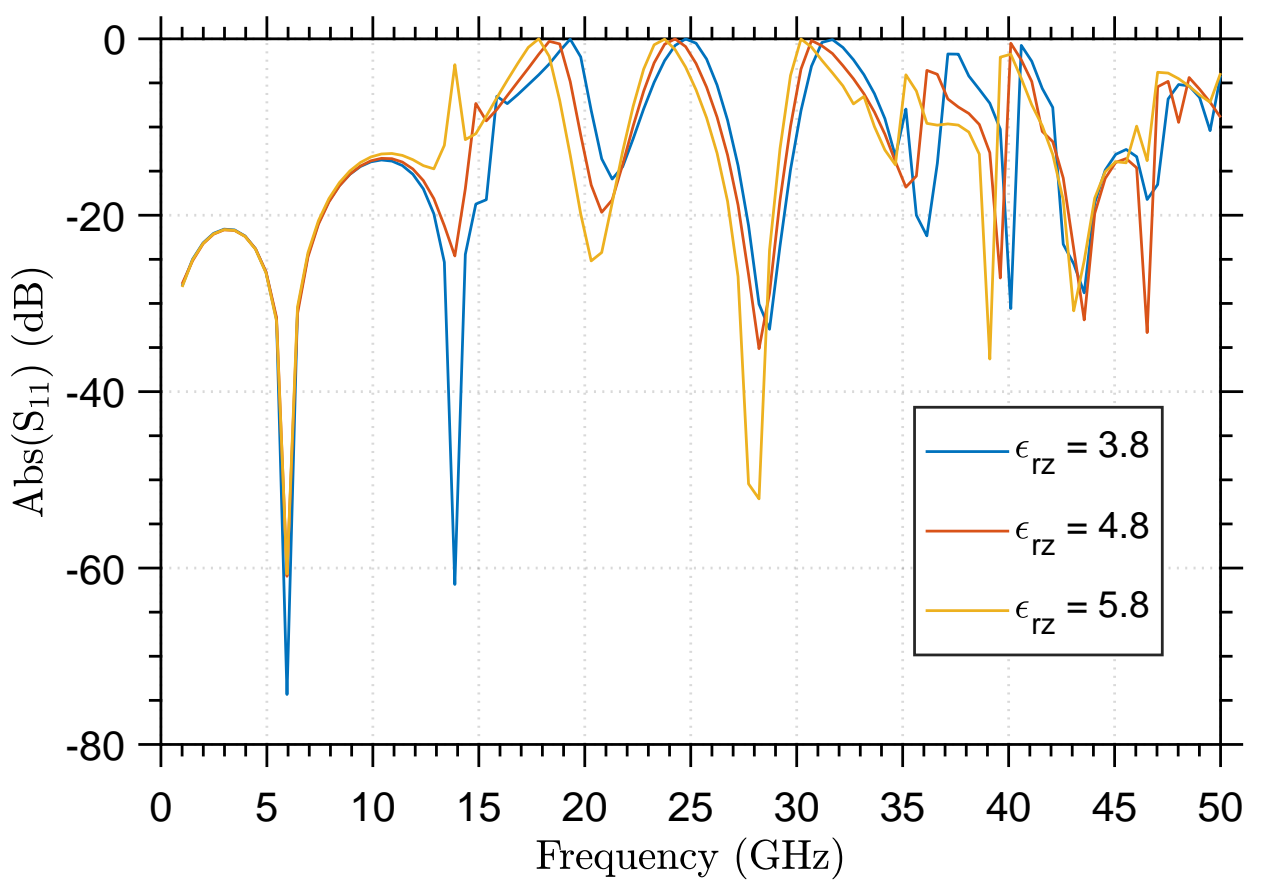

Figure 3.32: Reflection coefficient of structure 2 considering different levels of anisotropy.

\section{Structure 3}

A structure composed of two coaxial waveguides with a circular one in the middle is considered now. The intermediate waveguide (in region 2) has three radial layers, as depicted in Fig. 3.33 and with the parameters detailed in Table 3.18.

Fig. 3.34 shows a comparison of our results with the ones obtained numerically via FD approach. We noticed some discrepancies in amplitude of the reflection coefficient of the first mode TM in region 1. This happen near some resonances or near the cutoff frequencies were the deficiencies of FD-based solutions are well-know.

In this example, the reflection coefficient observed in Fig 3.34 is null (i.e., there is total reflection condition) below the cutoff frequency of the first propagating mode in region 2 . This occurs at about $9.4 \mathrm{GHz}$, with the $\mathrm{TM}_{01}$ mode. Other two TM modes starts propagating inside waveguide 2: $\mathrm{TM}_{02}$ at $21.78 \mathrm{GHz}$ and $\mathrm{TM}_{03}$ at $37.62 \mathrm{GHz}$. The $\mathrm{TM}_{01}$ of region 1 has cutoff at 47.03 $\mathrm{GHz}$. 


\begin{tabular}{ccccccccc}
\hline Region & $\rho_{0}(\mathrm{~mm})$ & $\rho_{1}(\mathrm{~mm})$ & $\rho_{2}(\mathrm{~mm})$ & $\rho_{3}(\mathrm{~mm})$ & $\epsilon_{r 1}$ & $\epsilon_{r 2}$ & $\epsilon_{r 3}$ & $\mathrm{~L}(\mathrm{~mm})$ \\
\hline 1 & 1.84 & 2.00 & 5.00 & - & 2.55 & 1 & - & $\infty$ \\
2 & 0 & 2.00 & 4.00 & 6.00 & 2.55 & 6 & 2.55 & 10 \\
3 & 1.84 & 2.00 & 5.00 & - & 2.55 & 1 & - & $\infty$ \\
\hline \multicolumn{7}{c}{$\sigma=10^{-6}(\mathrm{~S} / \mathrm{m})$} \\
\hline
\end{tabular}

Table 3.18: Dimensions and parameters of structure 3.

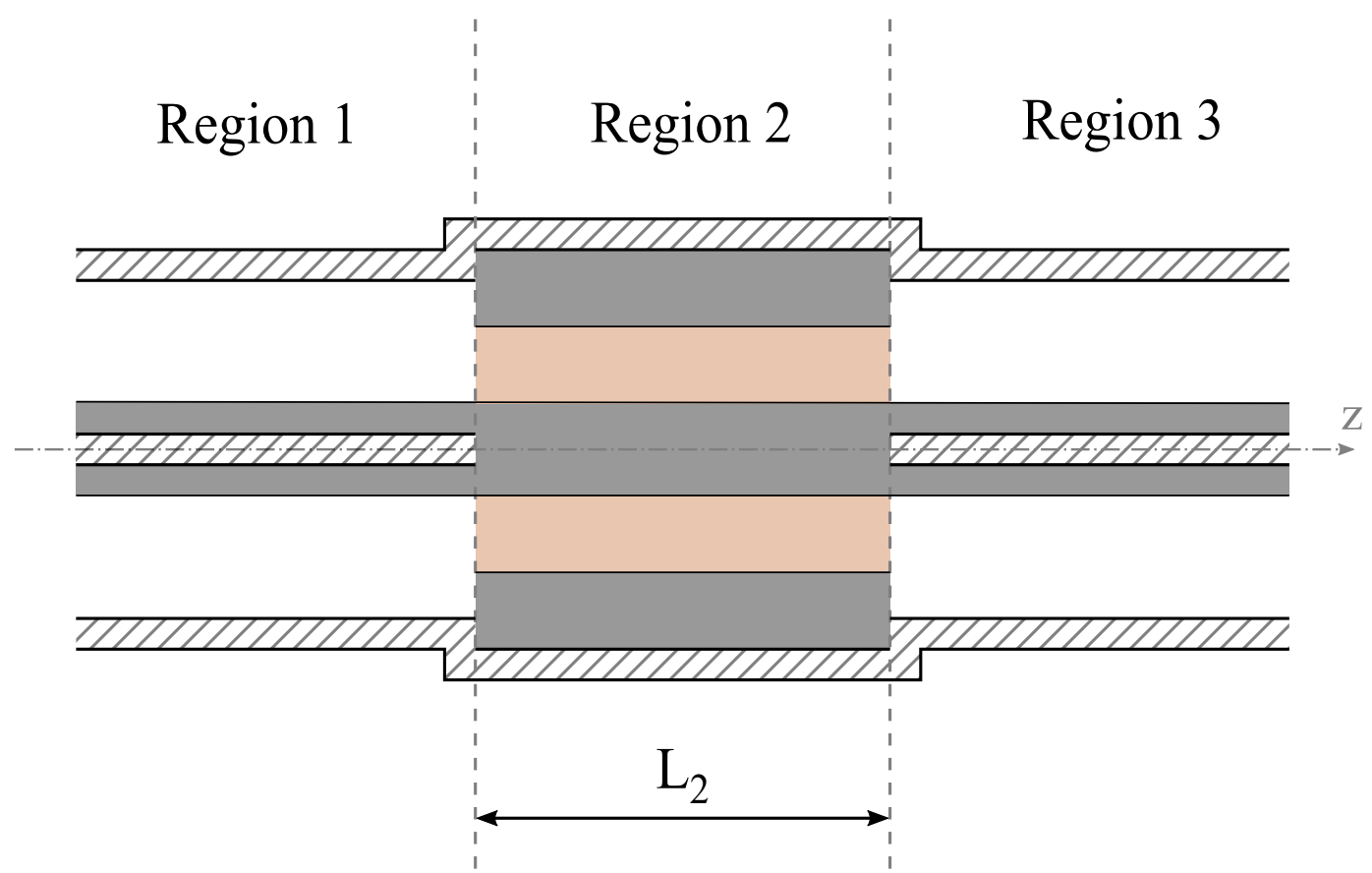

Figure 3.33: Geometry of structure 3.

A comparison on the reflection coefficient for different values of $\epsilon_{r z}$ is also done for structure 3. The middle layer in region 2 (colored layer in Fig 3.33) has $\epsilon_{r s}=6$ and $\epsilon_{r z}=\{5,6,7\}$. Fig 3.35 shows the $S_{11}(d B)$ curve for this example. The same observation made for structure 2 apply here, it is not possible observe a clear relation between the reflection coefficient and the anisotropy of the material. 


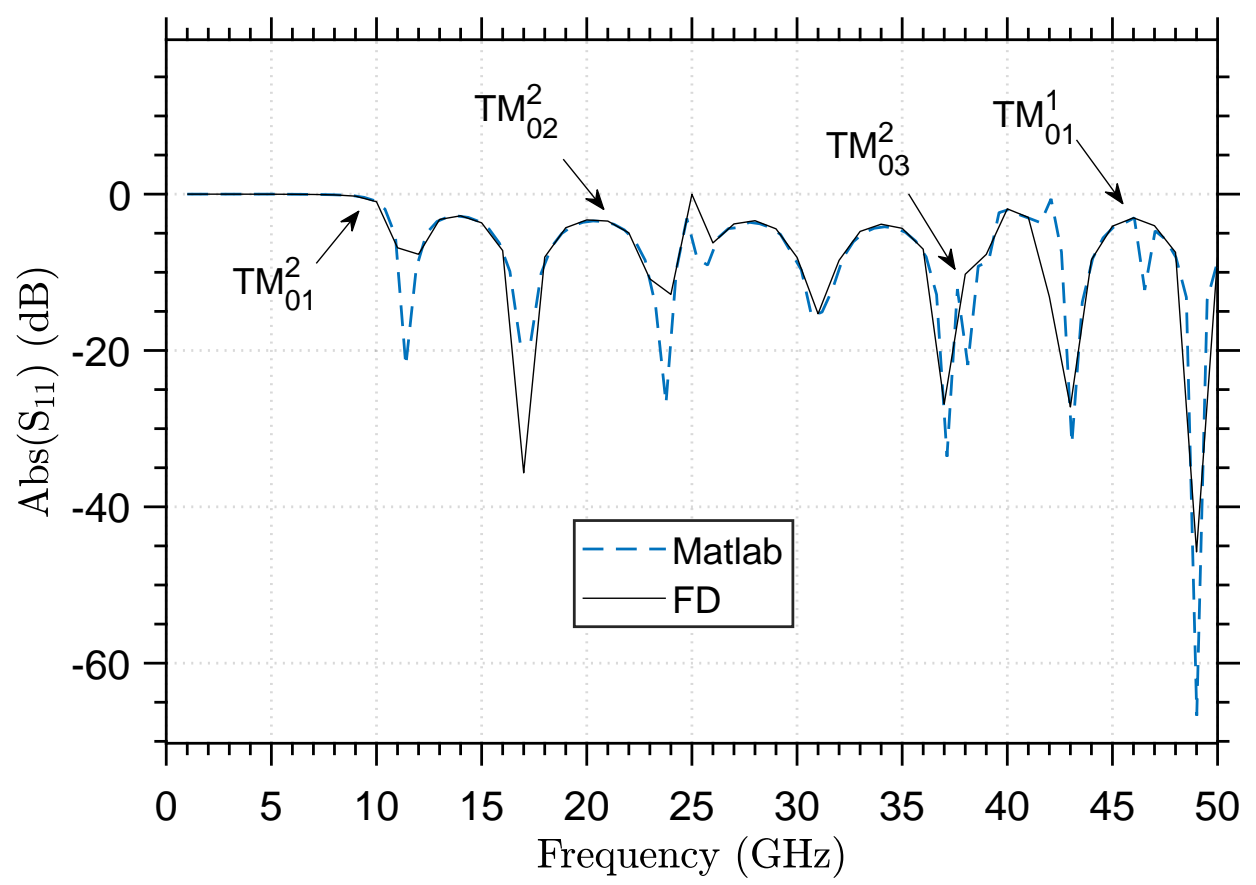

Figure 3.34: Reflection coefficient of structure 3.

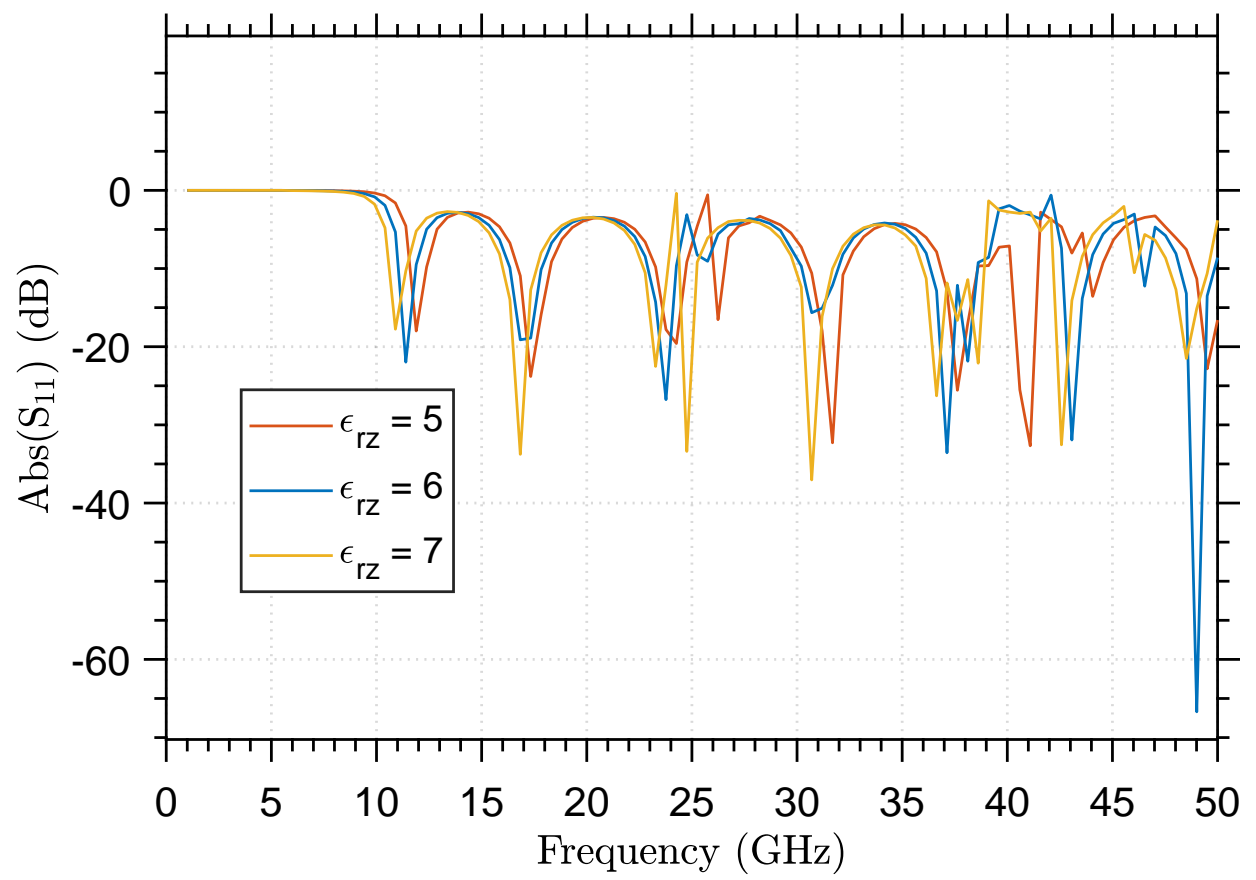

Figure 3.35: Reflection coefficient of structure 3 considering different levels of anisotropy.

\section{Structure 4}

In order to test the versatility and robustness of the presented mathematical formulation and the associated numerical algorithm, we finally consider 
a structure formed by the three circular waveguides. All the waveguides are homogeneous, with only a single radial layer, and 20 modes were considered. Notice that only a couple of modes are necessary for convergence, but we have employed a large number of then to test the stability and convergence of the semi-analytical MMT-based approach we wave introduce in this work. The numerical results are shown in Fig 3.37. In this case, we observed more discrepancies with the curve obtained via FD, mainly in the higher frequencies. To reduce the computational time spent in the numerical method, a small number of frequencies were used to calculate the reflection coefficient in comparison with the number used in Matlab. This may have caused the observed differences. Despite that, the solutions agree very well.

This structure is an extension of junction 12. The two waveguides of this junction were coupled with another waveguide equal to the guide from region 1 . Then, the same cutoffs detailed for junction 12 will be found here. At $23.17 \mathrm{GHz}$, the $\mathrm{TM}_{01}$ mode becomes propagating inside region 1, and as a consequence, the power transmission along this structure is only possible above the said frequency.

\begin{tabular}{ccccc}
\hline Region & $\rho_{0}(\mathrm{~mm})$ & $\rho_{1}(\mathrm{~mm})$ & $\epsilon_{r 1}$ & $\mathrm{~L}(\mathrm{~mm})$ \\
\hline 1 & 0 & 5.00 & 1 & $\infty$ \\
2 & 0 & 6.00 & 1 & 10 \\
3 & 0 & 5.00 & 1 & $\infty$ \\
\hline \multicolumn{5}{c}{$\sigma=10^{-6}(\mathrm{~S} / \mathrm{m})$} \\
\hline
\end{tabular}

Table 3.19: Dimensions and parameters of structure 4 .

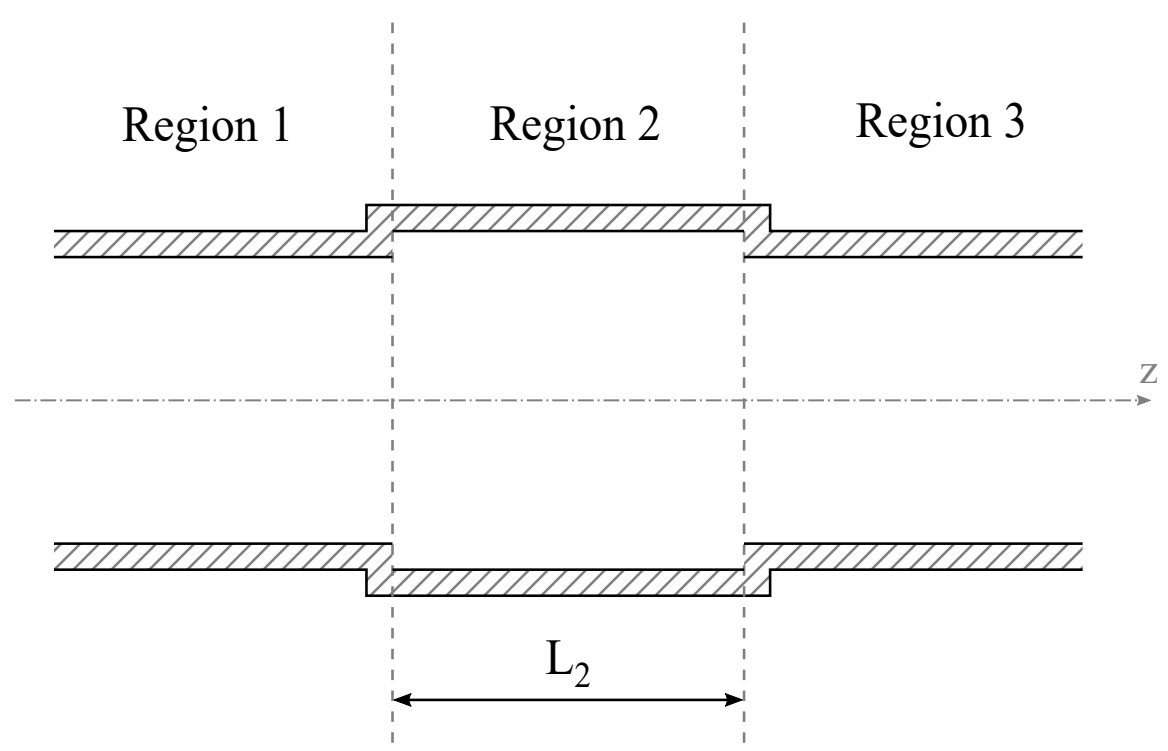

Figure 3.36: Geometry of structure 4. 


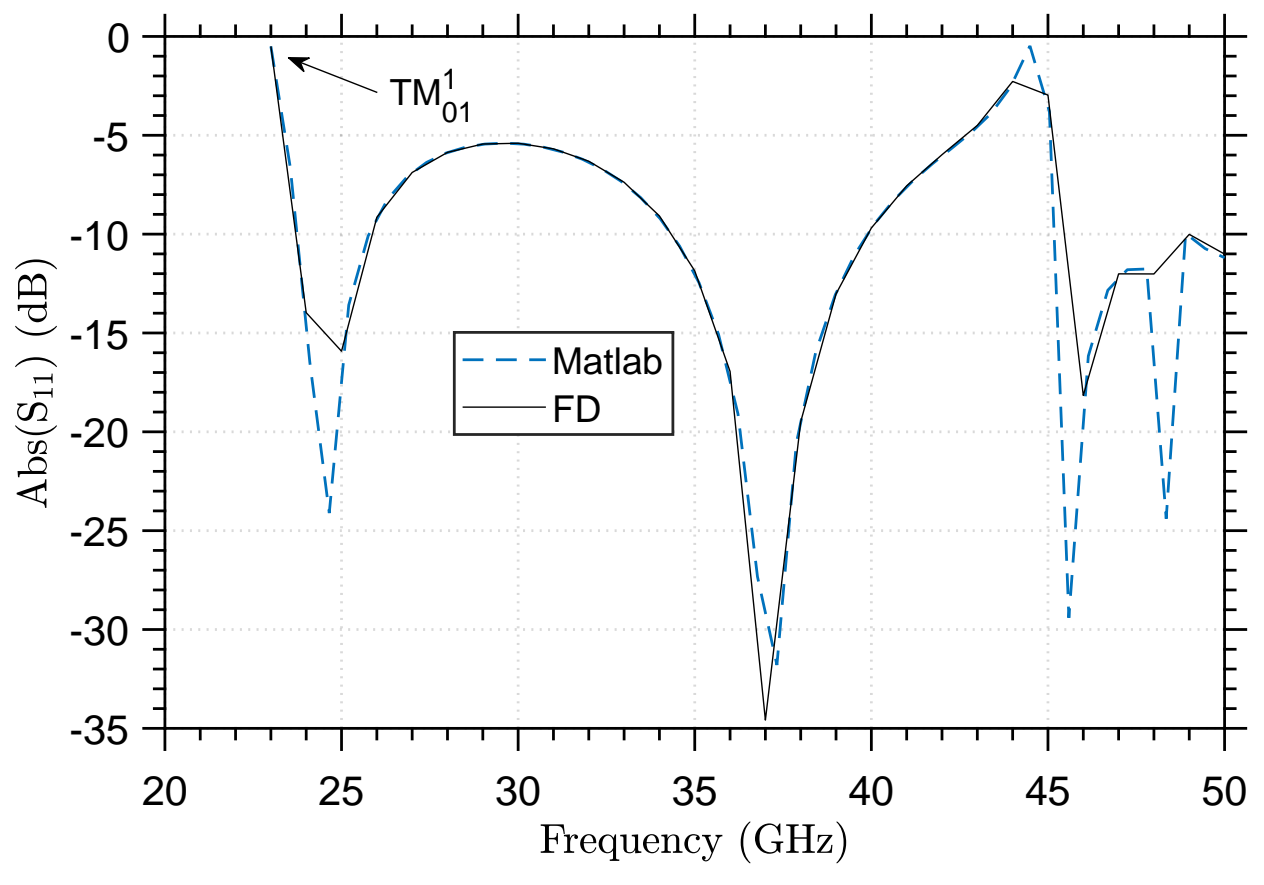

Figure 3.37: Reflection coefficient of structure 4. 


\section{4}

\section{Conclusion}

In this final chapter, we will review and resume the topics addressed in this work tracing a parallel with the main results presented previously.

We started discussing in Chapter 1 the relevance of inhomogeneous, anisotropic, and lossy materials to applied electromagnetism and microwave system applications. As the interest and the use of such complex materials increase, more studies had to be carried out about computational electromagnetic models and its potential applications to the development of novel technologies. The object of study of this dissertation was about semi-analytical methodologies for modeling structures composed by junctions of waveguides filled with complex media. Specifically, our main focus was on coaxial and circular radially-layered waveguides excited by azimuthally-symmetric fields.

The mode-matching technique was suggested to deal with the structure of interest due to its low-cost computational resources if compared to other methodologies. Numerical methods for the discretization of Maxwell's equations based on finite-difference or finite-elements allow us to analyze complexshaped geometries, but accurate solutions generally demand a large amount of $\mathrm{CPU}$ time and RAM memory that can become prohibitive in an optimization process.

The MMT-based solution we have proposed in this work employs a subdomain decomposition of the original multi-waveguide problem. This allowed us to calculate the generalized scattering matrix of each junction via closedform equations, and then, the entire structure composed of several junctions could be characterized by a simple cascading procedure.

The derivation of the electromagnetic fields and its characteristic equations was done in Chapter 2. We presented expressions for modeling radiallylayered waveguides, including the special cases of homogeneous circular and coaxial waveguides. For the homogeneous guides, a root-finding procedure in the $k_{\rho}$ complex-plane was introduced. In the case of inhomogeneous media, the winding number method was applied for determining the number of modal eigenvalues that are inside a region on the complex plane. Our region of the search was defined differently for two cases: a) when the media is lossy, we consider an attenuation factor, and b) when is lossless, we use alternative 
estimator for the number of modes to delimit the region of search. Finally, in order to effectively determine the eigenvalues, the Muller method was employed. This elaborated procedure ensures that all modal fields of interest will be included in the solutions, conferring robustness to our method.

The MMT was presented in Chapter 3, where the development of the reaction integrals for dealing the coupling between hybrid, TE, and TM modal fields was shown. In addition, we have presented a novel set of coupling integrals associated to the TEM mode.

A numerical algorithm was implemented in the Matlab platform in order to validate the capacities of the presented mathematical model. The simulation results obtained with our approach were compared versus those from the MMT in [33], and the FE and FD obtained using the CST Studio Suite.

Firstly, coaxial and circular waveguides were studied with different filling profiles to corroborate our algorithm. Excellent agreement was observed in all availed problems. It was possible to verify that fields concentrate in the layers with higher electric permittivity. Two factors contribute to a higher intensity of the field in these layers, the value of $\epsilon$ and the frequency. As one of these aspects increased, the concentration of the fields becomes more pronounced as well.

We also presented a comparison between the four described methods to calculate the winding number. In practical terms, they all conferred similar results, but some differences in the required CPU time was noticed. Although, the parallelization on computing the integrals can be explored in order to improve the proposed modified method.

Subsequently, numerical results on the applications of our mode-matching solution were presented. Simple junctions of two coaxial waveguides were exposed to ensure the convergence of our method. We showed that the numerical algorithm developed allowed us to numerically approximate a heterogeneous waveguide to a homogeneous one, proving that our procedure was robust enough to represent different scenarios.

Several examples of simple junctions of coaxial, circular, and radiallystratified waveguide structures were explored. To the best of our knowledge, this was the first time that the mode coupling between arbitrary radiallymultilayered waveguides was analyzed via a closed-form GSM formalism. The numerical results obtained using the proposed methodology were with good agreement when compared with reference solutions. It should be observed that junctions of coaxial to circular and circular to circular waveguides are special cases that were also examined by our algorithm. It was possible to observe the influence and importance of the higher-order modes for the solutions and how 
their cutoff frequencies change with the characteristics of the material filling the waveguides. The curves of the reflection coefficient $\left(S_{11}\right)$ in decibel of the dominant mode versus the frequency are widely used to determine the complex parameters of materials in measurement cells, and for this reason, this type of characterization is a relevant engineering application for the presented model.

The scientific contribution of this work relays on the application of the MMT to junctions of waveguides with a different number of radial layers, that may be composed of inhomogeneous, anisotropic, and lossy materials. The results presented here for the fields inside generic circular and coaxial waveguide and junctions with different number of layers via a closed-form MMT-based method was not found before in the literature. Also, the modifications on the calculation of the winding number described in Chapter 2 introduce novel aspects to find the number of eigenmodes in cylindrically-stratified waveguides. In addition, we found expressions for calculating the coupling integrals for homogeneous coaxial waveguides, which includes the interactions of TE, TM, and hybrid modes with the transmission-line TEM mode.

For future works, we recommend the implementation of the equations developed for the reaction integrals in the homogeneous cases, present in Chapter 2. These expressions will enables us to uses as input data a one layer coaxial waveguide, overcoming the numerical issues caused by the Hankel function when its argument vanishes. The approximations made in the validation of the mode-matching method for guides with similar layers to simulate a homogeneous one will no more be necessary and will reduce computational time.

It is also of interest to future researches of the methodology presented here for determining the parameters of sample materials through an inverse process, as done similarly in $[35,36,39]$. A coaxial or circular characterization cell filled with the sample under test can be used to obtain reflection and transmission S parameters. Using these data obtained experimentally, an inversion technique can be used for obtaining the complex-valued constitutive parameters $\epsilon$ and $\mu$ of the sample. 


\section{Bibliography}

[1] C. A. Balanis, Advanced engineering electromagnetics. John Wiley \& Sons, 1999.

[2] V. Kamra and A. Dreher, "Analysis of anisotropic inhomogeneous dielectric waveguides with discrete mode matching method," in 2019 IEEE MTT-S International Microwave Symposium (IMS), 2019, pp. 24-27.

[3] A. Novitsky, A. S. Shalin, and A. Lavrinenko, "Analytical solutions for waves in spherically-and cylindrically-symmetric inhomogeneous media," in 2017 11th International Congress on Engineered Materials Platforms for Novel Wave Phenomena (Metamaterials). IEEE, 2017, pp. 235-237.

[4] F. Teixeira and W. Chew, "Cylindrical 3d pml-plrc-fdtd schemes for frequencydispersive and inhomogeneous media," in IEEE Antennas and Propagation Society International Symposium. 1999 Digest. Held in conjunction with: USNC/URSI National Radio Science Meeting (Cat. No. 99CH37010), vol. 2. IEEE, 1999, pp. 1304-1307.

[5] D. Bhattacharya, B. Ghosh, and K. Sarabandi, "Evaluation of efficient closedform green's function in a cylindrically stratified medium," IEEE Transactions on Antennas and Propagation, vol. 65, no. 3, pp. 1505-1510, 2017.

[6] A. Alexandrin, "Implementation of a radially inhomogeneous medium and construction of the aperture antennas on its basis," in 2013 International Siberian Conference on Control and Communications (SIBCON). IEEE, 2013, pp. 1-4.

[7] K. Sainath and F. L. Teixeira, "Interface-flattening transform for em field modeling in tilted, cylindrically stratified geophysical media," IEEE Antennas and Wireless Propagation Letters, vol. 13, pp. 1808-1811, 2014.

[8] I. Shipilova, O. Suvorova, Y. F. Filipov, and Y. V. Prokopenko, "Radially three-layered dielectric resonators for millimeter wavelengths," in 2007 International Kharkov Symposium Physics and Engrg. of Millimeter and SubMillimeter Waves (MSMW), vol. 2. IEEE, 2007, pp. 663-665. 
[9] J. Liu, N. Liu, and Q. H. Liu, "Spectral numerical mode matching method for 3d layered multi-region structures," IEEE Transactions on Antennas and Propagation, 2019.

[10] X. Wang, H. Zhang, and C. Ying, "Acoustic field in a borehole within a horizontally layered formation," in 1995 IEEE Ultrasonics Symposium. Proceedings. An International Symposium, vol. 2. IEEE, 1995, pp. 11211124.

[11] M. W. Hyde IV, M. J. Havrilla, and A. E. Bogle, "Nondestructive determination of the permittivity tensor of a uniaxial material using a two-port clamped coaxial probe," IEEE Transactions on Microwave Theory and Techniques, vol. 64, no. 1, pp. 239-246, 2015.

[12] Y. Lugovtsova, J. Bulling, C. Boller, and J. Prager, "Analysis of guided wave propagation in a multi-layered structure in view of structural health monitoring," Applied Sciences, vol. 9, no. 21, p. 4600, 2019.

[13] G. S. Rosa, M. S. Canabarro, J. R. Bergmann, and F. L. Teixeira, "A comparison of two numerical mode-matching methodologies for the analysis of inhomogeneous media with radial and vertical stratifications," IEEE Transactions on Antennas and Propagation, vol. 66, no. 12, pp. 7499-7504, 2018.

[14] L. Saavedra, G. S. Rosa, and J. R. Bergmann, "A novel semi-analytical modematching technique for modeling realistic electromagnetic well-logging sensors immersed in dissipative inhomogeneous media," in 2019 URSI International Symposium on Electromagnetic Theory (EMTS). IEEE, 2019, pp. 1-4.

[15] _ - "An accurate technique for modeling realistic well-logging sensors inside complex media," in 2019 International Applied Computational Electromagnetics Society Symposium (ACES). IEEE, 2019, pp. 1-2.

[16] D. Hong, S. Yang, Y. Zhang, W.-F. Huang, and Q. H. Liu, "Pseudoanalytical formulations for modeling the effect of an insulating layer in electromagnetic well logging," IEEE Transactions on Geoscience and Remote Sensing, vol. 56, no. 12, pp. 7022-7029, 2018.

[17] Y. Tian, D. Zhou, S. Kong, W. Zhang, B. Li, and J. Qi, "Modeling and simulation on three-layer media propagation characteristics of underwater electromagnetic field in shallow sea area," in 2019 IEEE 4th Advanced Information Technology, Electronic and Automation Control Conference (IAEAC), vol. 1, 2019, pp. 1515-1518. 
[18] V. Kamra and A. Dreher, "Analysis of circular and noncircular waveguides and striplines with multilayered uniaxial anisotropic medium," IEEE Transactions on Microwave Theory and Techniques, vol. 67, no. 2, pp. 584-591, 2018.

[19] — - "Full-wave equivalent circuit for the analysis of multilayered microwave structures with anisotropic layers," Electronics Letters, vol. 54, no. 3, pp. 153-155, 2017.

[20] S. Yang, D. Hong, W.-F. Huang, and Q. H. Liu, "A stable analytic model for tilted-coil antennas in a concentrically cylindrical multilayered anisotropic medium," IEEE Geoscience and Remote Sensing Letters, vol. 14, no. 4, pp. 480-483, 2017.

[21] L. Sun, "Computations of electromagnetic wave scattering from anisotropic and inhomogeneous objects using volume integral equation methods," in 2015 IEEE International Symposium on Antennas and Propagation \& USNC/URSI National Radio Science Meeting. IEEE, 2015, pp. 1820-1821.

[22] J. Dai and Q. H. Liu, "Efficient computation of electromagnetic waves in anisotropic orthogonal-plano-cylindrically layered media using the improved numerical mode matching ( $\mathrm{nmm}$ ) method," IEEE Transactions on Antennas and Propagation, vol. 63, no. 8, pp. 3569-3578, 2015.

[23] _ - "Fast computation of electromagnetic fields in anisotropic media layered both vertically and cylindrically using the numerical mode matching (nmm) method," in 2013 USNC-URSI Radio Science Meeting (Joint with AP-S Symposium). IEEE, 2013, pp. 142-142.

[24] R. Kotyński, T. J. Antosiewicz, M. Stolarek, and A. Pastuszczak, "Layered and core-shell uniaxial absorbers," in 2014 16th International Conference on Transparent Optical Networks (ICTON). IEEE, 2014, pp. 1-2.

[25] G. S. Rosa and J. R. Bergmann, "Electromagnetic propagation along lossy anisotropic and radially stratified cylindrical structures," in 2014 Loughborough Antennas and Propagation Conference (LAPC). IEEE, 2014, pp. 155159.

[26] F. L. Teixeira and W. C. Chew, "Finite-difference computation of transient electromagnetic waves for cylindrical geometries in complex media," IEEE Transactions on geoscience and remote sensing, vol. 38, no. 4, pp. 15301543, 2000. 
[27] E. Y. Smolkin, "On the problem of propagation of nonlinear coupled te-tm waves in a double-layer nonlinear inhomogeneous cylindrical waveguide," in 2015 Days on Diffraction (DD). IEEE, 2015, pp. 1-5.

[28] — - "Propagation of te waves in a double-layer nonlinear inhomogeneous cylindrical waveguide," in Proceedings of the International Conference Days on Diffraction 2014. IEEE, 2014, pp. 204-208.

[29] N. Li, D.-C. Hong, H.-M. Liu, W. Han, T. Chen, and J.-T. He, "An analytic algorithm to solve electromagnetic field in planar-stratified biaxial anisotropic medium," in 2019 IEEE International Conference on Computational Electromagnetics (ICCEM). IEEE, 2019, pp. 1-3.

[30] E. Smolkin, M. Snegur, and Y. Shestopalov, "Numerical method for electromagnetic wave propagation problem in a cylindrical anisotropic inhomogeneous waveguide with longitudinal magnetization," in 2017 Progress in Electromagnetics Research Symposium-Fall (PIERS-FALL). IEEE, 2017, pp. 299-305.

[31] E. Smolkin, "Numerical method for electromagnetic wave propagation problem in a cylindrical inhomogeneous metal dielectric waveguiding structures," Mathematical Modelling and Analysis, vol. 22, no. 3, pp. 271-282, 2017.

[32] G. S. Rosa and J. R. Bergmann, "An efficient mode-matching solution for open-ended coaxial waveguides via analytic continuation of the radial space," in 2018 IEEE International Symposium on Antennas and Propagation \& USNC/URSI National Radio Science Meeting. IEEE, 2018, pp. 721-722.

[33] G. S. Rosa, "Propagação de ondas eletromagnéticas em estruturas coaxiais carregadas com meios nao homogêneos excitadas pelo modo tem," Master Thesis, Dept. Elect. Eng., Pontifical Catholic University of Rio de Janeiro, Rio de Janeiro, RJ, Brasil, 2013.

[34] N. Yashina, A. Brovenko, N. Melezhik, P. Melezhik, A. Poyedinchuk, and O. Troshchylo, "1d inverse problems of electromagnetic theory of layered inhomogeneous anisotropic media," in 2017 XXIInd International Seminar/Workshop on Direct and Inverse Problems of Electromagnetic and Acoustic Wave Theory (DIPED). IEEE, 2017, pp. 118-122.

[35] N. E. Belhadj-Tahar, A. Fourrier-Lamer, and H. De Chanterac, "Broadband simultaneous measurement of complex permittivity and permeability using a coaxial discontinuity," IEEE Transactions on Microwave Theory and Techniques, vol. vol. 38, no. n. 1, pp. 1-7, 1990. 
[36] J. Huang, K. Wu, P. Morin, and C. Akyel, "Characterization of highly dispersive materials using composite coaxial cells: electromagnetic analysis and wideband measurement," IEEE Transactions on Microwave Theory and Techniques, vol. vol. 44, no. n. 5, pp. 770-777, 1996.

[37] W. C. Chew, K. J. Olp, and G. P. Otto, "Design and calibration of a large broadband dielectric measurement cell," IEEE Transactions on Geoscience and Remote Sensing, vol. vol. 29, no. n. 1, pp. 42-47, 1991.

[38] V. V. Thalakkatukalathil, "Electromagnetic modeling and characterization of anisotropic ferrite materials for microwave isolators/circulators," PhD. Thesis, Université de Bretagne occidentale, Brest, France, 2017.

[39] N.-E. Belhadj-Tahar and A. Fourrier-Lamer, "Broad-band simultaneous measurement of the complex permittivity tensor for uniaxial materials using a coaxial discontinuity," IEEE Transactions on Microwave Theory and Techniques, vol. vol. 39, no. n. 10, pp. 1718-1724, 1991.

[40] C. Legrand and S. Toutain, "Automatic permittivity measurements in a wide frequency range: Application to anisotropic fluids," IEEE Transactions on Microwave Theory and Techniques, vol. 30, no. 11, pp. 2015-2017, 1982.

[41] E. Marouby, M. Aubourg, and P. Guillon, "Application of the finite element method to the design of transitions between coaxial lines," in IEE Proceedings H-Microwaves, Antennas and Propagation, vol. 137. IET, 1990, pp. 219-225.

[42] W. Scott, "Accurate modeling of axisymmetric two-port junctions in coaxial lines using the finite element method," IEEE Transactions on Microwave Theory and Techniques, vol. 40, no. 8, pp. 1712-1716, 1992.

[43] W. Yu, R. Mittra, and S. Dey, "Application of the nonuniform fdtd technique to analysis of coaxial discontinuity structures," IEEE Transactions on Microwave Theory and Techniques, vol. 49, no. 1, pp. 207-209, 2001.

[44] W. K. Gwarek, "Computer-aided analysis of arbitrarily shaped, coaxial discontinuities," IEEE transactions on microwave theory and techniques, vol. 36, no. 2, pp. 337-342, 1988.

[45] A. Nicolson and G. Ross, "Measurement of the intrinsic properties of materials by time-domain techniques," IEEE Transactions on instrumentation and measurement, vol. 19, no. 4, pp. 377-382, 1970.

[46] M. N. Afsar, J. R. Birch, R. Clarke, and G. Chantry, "The measurement of the properties of materials," Proceedings of the IEEE, vol. 74, no. 1, pp. 183-199, 1986. 
[47] N.-E. Belhadj-Tahar and A. Fourrier-Lamer, "Broad-band analysis of a coaxial discontinuity used for dielectric measurements," IEEE Transactions on Microwave Theory and Techniques, vol. vol. 34, no. n. 3, pp. 346-350, 1986.

[48] R. Huang and D. Zhang, "Application of mode matching method to analysis of axisymmetric coaxial discontinuity structures used in permeability and/or permittivity measurement," Progress In Electromagnetics Research, vol. 67, pp. 205-230, 2007.

[49] E. Smolkin, M. Snegur, and Y. Shestopalov, "Hybrid waves in a cylindrical anisotropic inhomogeneous metal-dielectric waveguide," in 2019 Photonlcs \& Electromagnetics Research Symposium-Spring (PIERS-Spring). IEEE, 2019, pp. $1757-1764$.

[50] H. Cui and B. Zhang, "Guided waves in cylindrical multi-layered medium," in 2008 IEEE Ultrasonics Symposium. IEEE, 2008, pp. 912-915.

[51] G. S. Rosa, "Pseudo-analytical modeling for electromagnetic well- logging tools in complex geophysical formations," PhD Thesis, Dept. Elect. Eng., Pontifical Catholic University of Rio de Janeiro, Rio de Janeiro, RJ, Brasil, 2017.

[52] N. Marcuvitz, Waveguide handbook. let, 1951, no. 21.

[53] A. Puzella and A. Palevsky, "Electromagnetic dispersion of a coaxial waveguide with an arbitrary radial dielectric profile," IEEE Transactions on Electronic Devices, vol. vol. 35, no. n. 11, pp. 2048-2051, 1988.

[54] R. E. Mclntosh and L. J. Turgeon, "Propagation along transversely inhomogeneous coaxial transmission lines (short papers)," IEEE Transactions on Microwave Theory and Techniques, vol. vol. 21, no. n. 3, pp. 139-142, 1973.

[55] R. Yamada and K. Watanabe, "Propagation in cylindrical waveguide containing inhomogeneous dielectric," IEEE Transactions on Microwave Theory and Techniques, vol. vol. 13, no. n. 5, pp. 716-717, Sep. 1965.

[56] W. C. Chew, Waves and Fields in Inhomogeneous Media. IEEE press, 1995.

[57] R. F. Harrington, Time-harmonic electromagnetic fields. New York, NY, USA: McGraw-Hill, 1961.

[58] E. Sorolla, J. R. Mosig, and M. Mattes, "Algorithm to calculate a large number of roots of the cross-product of bessel functions," IEEE Transactions on Antennas and Propagation, vol. 61, no. 4, pp. 2180-2187, 2013. 
[59] G. S. Rosa, J. R. Bergmann, and S. R. Zang, "Improvements on the search modal propagation constants in lossy circular waveguides," IEEE Antennas and Wireless Propagation Letters, vol. 13, pp. 754-757, 2014.

[60] P. Kowalczyk, "Global complex roots and poles finding algorithm based on phase analysis for propagation and radiation problems," IEEE Transactions on Antennas and Propagation, vol. 66, no. 12, pp. 7198-7205, 2018.

[61] J. W. Brown, R. V. Churchill et al., Complex variables and applications. Boston: McGraw-Hill Higher Education,, 2009.

[62] T. Johnson and W. Tucker, "Enclosing all zeros of an analytic function-a rigorous approach," Journal of Computational and Applied Mathematics, vol. 228, no. 1, pp. 418-423, 2009.

[63] G. S. Rosa, J. R. Bergmann, and F. L. Teixeira, "A robust mode-matching algorithm for the analysis of triaxial well-logging tools in anisotropic geophysical formations," IEEE Trans. Geosci. Remote Sens., vol. 55, no. 5, pp. 2534-2545, May 2017.

[64] G. S. Rosa, J. R. Bergmann, and F. L. Teixeira, Full-Wave Pseudoanalytical Methods for Cylindrically Symmetric Electromagnetic Structures: Applications in Sensing and Telemetry for Geophysical Exploration. World Scientific Publishing Co Pte Ltd, 2020, ch. 10, pp. 385-431.

[65] P. Kowalczyk and W. Marynowski, "Efficient complex root tracing algorithm for propagation and radiation problems," IEEE Transactions on Antennas and Propagation, vol. 65, no. 5, pp. 2540-2546, 2017.

[66] N. S. Brannen and B. Ford, "Logarithmic differentiation: Two wrongs make a right," The College Mathematics Journal, vol. vol. 35, no. n. 5, pp. 388-390, 2004.

[67] M. Abramowitz and I. A. Stegun, Handbook of Mathematical Functions. New York, NY, USA: Dover Publications, 1964.

[68] MATLAB, version 9.8.0.1323502 (R2020a). Natick, Massachusetts: The MathWorks Inc., 2020.

[69] R. Yamada and K. Watanabe, "Propagation in cylindrical waveguide containing inhomogeneous dielectric," IEEE Transactions on Microwave Theory and Techniques, vol. 13, no. 5, pp. 716-717, 1965. 
[70] M. Warecka, R. Lech, and P. Kowalczyk, "Efficient finite element analysis of axially symmetrical waveguides and waveguide discontinuities," IEEE Transactions on Microwave Theory and Techniques, vol. 67, no. 11, pp. 42914297, 2019.

[71] J. Wang, J. Tao, and F. Benmohamed, "An efficient numerical method to analyze discontinuity of coaxial cable," in 2017 International Applied Computational Electromagnetics Society Symposium-Italy (ACES). IEEE, 2017, pp. 1-2.

[72] M. A. Gnatyuk and V. M. Morozov, "An integral-equation analysis of waveguide step discontinuities," in 2018 IEEE 17th International Conference on Mathematical Methods in Electromagnetic Theory (MMET), 2018, pp. 209-212.

[73] G. S. Rosa, J. R. Bergmann, and F. L. Teixeira, "Axial mode-matching technique for analysis of directional well-logging sensor tools," in 2016 IEEE International Symposium on Antennas and Propagation (APSURSI). IEEE, 2016, pp. 2041-2042.

[74] M. S. Canabarro, G. S. Rosa, J. R. Bergmann, and F. L. Teixeira, "Some recent improvements in modeling of electromagnetic well-logging sensors via numerical mode-matching," in 2017 SBMO/IEEE MTT-S International Microwave and Optoelectronics Conference (IMOC). IEEE, 2017, pp. 1-5.

[75] G. S. Rosa and J. R. Bergmann, "Pseudo-analytical modeling for the electromagnetic propagation in stratified cylindrical structures," IEEE Antennas and Wireless Propagation Letters, vol. 15, pp. 344-347, 2016.

[76] A. Morini and T. Rozzi, "On the definition of the generalized scattering matrix of a lossless multiport," IEEE Transactions on Microwave Theory and Techniques, vol. vol. 49, no. n. 1, pp. 160-165, 2001.

[77] A. P. Orfanidis, G. A. Kyriacou, and J. N. Sahalos, "A mode-matching technique for the study of circular and coaxial waveguide discontinuities based on closed-form coupling integrals," IEEE Transactions on Microwave Theory and Techniques, vol. vol. 48, no. n. 5, pp. 880-883, 2000.

[78] A. S. Eleftheriades, G. V.; Omar and L. P. B. Katehi, "Some important properties of waveguide junction generalized scattering matrices in the context of the mode matching technique," IEEE Transactions on Microwave Theory and Techniques, vol. vol. 42, no. n. 10, pp. 1896-1903, 1994. 
[79] V. H. Rumsey, "Reaction concept in electromagnetic theory," Physical Review, vol. vol. 94, no. n. 6, p. 1483, 1954.

[80] C. Krowne, "Electromagnetic theorems for complex anisotropic media," IEEE Transactions on Antennas and Propagation, vol. vol. 32, no. n. 11, pp. 12241230, November 1984.

[81] A. L. dos Santos Lima, G. Simon da Rosa, and J. R. Bergmann, "A modematching solution for the study of cylindrical waveguide bifurcation via closedform coupling integrals," AEU - International Journal of Electronics and Communications, vol. 118, p. 153135, 2020.

[82] CST AG, CST Studio Suite 2016, Darmstadt, Germany, 2020.

[83] L.-F. Chen, C. Ong, C. Neo, V. Varadan, and V. K. Varadan, Microwave electronics: measurement and materials characterization. John Wiley \& Sons, 2004.

[84] L. C. Shen et al., "A laboratory technique for measuring dielectric properties of core samples at ultrahigh frequencies," Society of petroleum engineers journal, vol. 25, no. 04, pp. 502-514, 1985.

[85] J. Baker-Jarvis et al., "Measuring the permittivity and permeability of lossy materials: Solids, liquids, metals, and negative-index materials," National Institute of Standards and Technology, Tech. Rep., 2005, NIST Technical Note 1536. 